\title{
Conventional and High-Strength Headed Bars-Part 1: Anchorage Tests
}

\author{
by Krishna P. Ghimire, Yun Shao, David Darwin, and Matthew O'Reilly
}

Results of an experimental program on the anchorage strength of headed reinforcing bars are presented. Two hundred and two exterior beam-column joint specimens with concrete compressive strengths ranging from 3960 to 16,030 psi (27.3 to $110.6 \mathrm{MPa}$ ) were tested under monotonic loading. Key parameters included concrete compressive strength, embedment length, bar size, head size, spacing between headed bars, and confining reinforcement within the joint region. Bar stresses at failure ranged from 26,100 to 153,200 psi (180 to $1057 \mathrm{MPa}$ ). Specimens exhibited concrete breakout, side-face blowout, or a combination of the two failure modes, with concrete breakout being the dominant failure mode. A comparison of bar stress at anchorage failure with the stress calculated based on ACI 318-14 shows that ACI 318-14 provides a very conservative estimate of anchorage strength for No. 5 (No. 16) bars and low concrete compressive strengths. The estimate becomes progressively less conservative with increasing bar size and concrete compressive strength.

Keywords: anchorage; beam-column joints; bond and development; development length; headed bars; high-strength concrete; high-strength steel; reinforcement.

\section{INTRODUCTION}

Headed reinforcing bars are an alternative to hooked bars for end anchorage of reinforcing bars in concrete that provide a more efficient anchorage mechanism and limit congestion of the reinforcement. Design provisions for the development length of headed bars in ACI 318-14, "Building Code Requirements for Structural Concrete," however, impose more stringent restrictions on the application of headed bars than on hooked bars. For headed bars, the yield strength is limited to $60,000 \mathrm{psi}(420 \mathrm{MPa})$, and the concrete compressive strength is limited to $6000 \mathrm{psi}(40 \mathrm{MPa})$ when calculating the required development length, compared, respectively, to 80,000 and $10,000 \mathrm{psi}(555$ and $69 \mathrm{MPa})$ for hooked bars. Headed bars are also required to have a clear spacing between bars no less than four times the diameter of the bar $\left(4 d_{b}\right)$ and must conform to the Class HA head sizing requirements of ASTM A970-13a. These restrictions were imposed when headed bar provisions were adopted in the 2008 ACI 318 Code due to a lack of test data to support less restrictive requirements. ACI 318-14 also does not account for the contribution of confining reinforcement to the anchorage strength of headed bars.

The work described in this paper is part of a comprehensive study of the anchorage behavior of the headed bars (Shao et al. 2016; Ghimire et al. 2018) to identify the key factors that affect the development length of headed bars and to develop new design guidelines for development length that allow higher-strength steel and concrete to be used.
The results of the study are presented in a series of papers. This paper presents the experimental program, provides detailed information about the mode of failure of specimens, and evaluates the results, incorporating major parameters affecting the anchorage strength of the headed bars in simulated beam-column joints. The subsequent papers will present test results for other specimen types, analyze those test results, develop equations to characterize the anchorage strength of headed bars, and develop code change proposals that allow the use of headed bars in conjunction with a wider range of material properties than currently permitted.

\section{RESEARCH SIGNIFICANCE}

It is advantageous to use headed bars in reinforced concrete structures because they provide an efficient anchorage mechanism, reduce congestion, and facilitate easier fabrication, leading to lower construction costs. The construction industry, however, is not able to take optimal advantage of headed bars because the provisions in ACI 318-14 limit the use of headed bars and prevent the full benefits of high-strength reinforcing steel and concrete from being used. This study provides an expanded experimental database that can be used to improve the level of understanding of headed bar behavior and allow the development of improved code provisions, removing many of the current restrictions on headed bars.

\section{EXPERIMENTAL PROGRAM}

Two hundred and two simulated exterior beam-column joint specimens were tested to investigate the anchorage behavior of headed bars under monotonic loading (Shao et al. 2016). Key parameters included concrete compressive strength (3960 to 16,030 psi [ 27.3 to $110.6 \mathrm{MPa}$ ), embedment length (4 to 19.25 in. [102 to $499 \mathrm{~mm}$ ), bar size (No. 5, No. 8, and No. 11 [No. 16, No. 25, and No. 36]), head size (bearing areas from 3.8 to 15 times the bar area $A_{b}$ ), number of headed bars tested simultaneously in a specimen (two, three, or four), confining reinforcement within the joint region ranging from none to six No. 3 (No. 10) bars (spaced at three times the bar diameter $d_{b}$ ), and center-to-center spacing between the bars (2.9 to $\left.11.8 d_{b}\right)$. Based on differences in anchorage strength (Shao et al. 2016), headed bars were categorized as "widely spaced" if the centerto-center spacing between bars was $8 d_{b}$ or greater and "closely spaced" if the center-to-center spacing between bars was less

ACI Structural Journal, V. 116, No. 3, May 2019

MS No. S-2018-219, doi: 10.14359/51714479, received June 14, 2018, and reviewed under Institute publication policies. Copyright (C) 2019, American Concrete Institute. All rights reserved, including the making of copies unless permission is obtained from the copyright proprietors. Pertinent discussion including author's closure, if any, will be published ten months from this journal's date if the discussion is received within four months of the paper's print publication. 


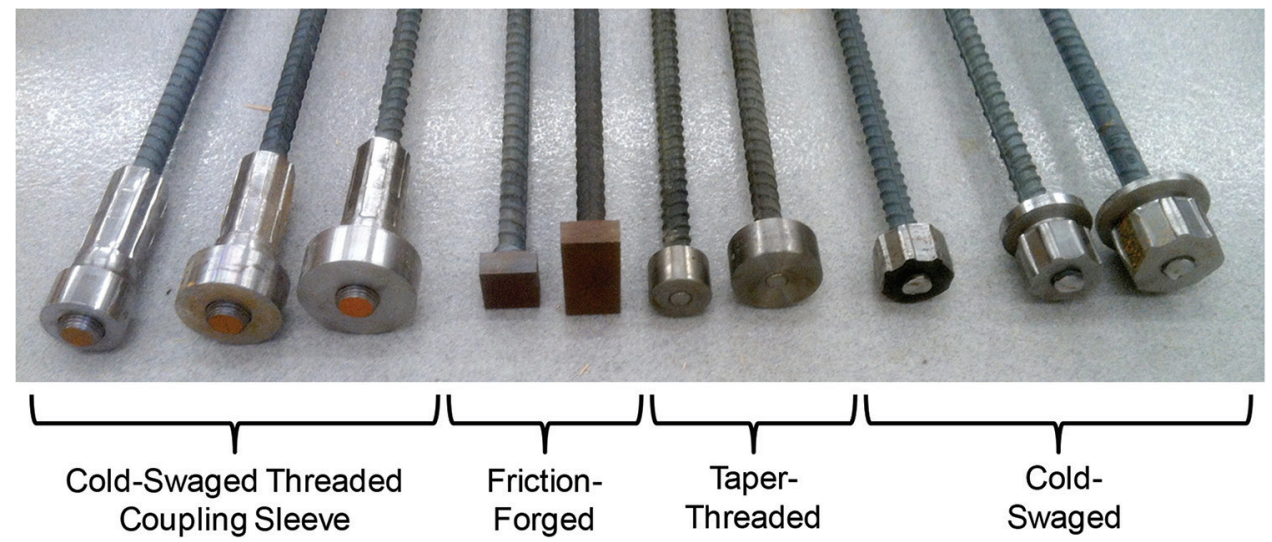

Fig. 1-No. 8 (No. 25) headed bars included in this study. (Left to right: cold-swaged threaded coupling sleeve, friction-forged, taper-threaded, and cold-swaged.)

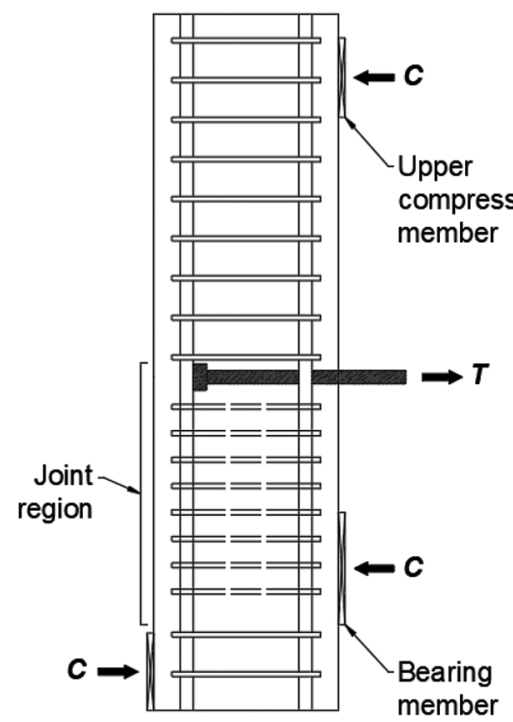

(a)

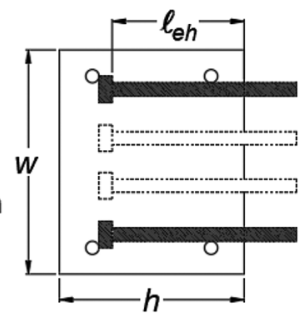

(b)

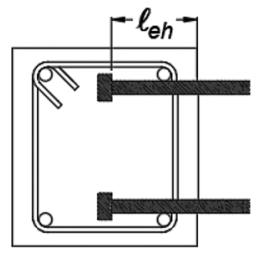

(d)

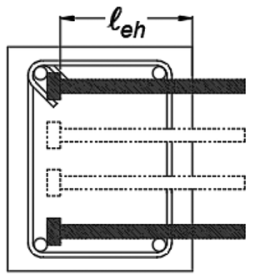

(c)

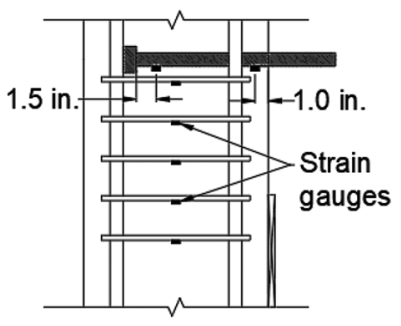

(e)

Fig. 2-Schematic of specimen: (a) side view of specimen; (b) cross section of specimen without confining reinforcement; (c) cross section of specimen with confining reinforcement; (d) cross section of specimen with headed bars anchored at middepth of column and with confining reinforcement; and (e) location of strain gauges on headed bars and confining reinforcement. (Note: $1 \mathrm{in} .=25.4 \mathrm{~mm}$.)

than $8 d_{b}$. Bar stresses at anchorage failure ranged from 26,100 to $153,200 \mathrm{psi}$ (180 to $1057 \mathrm{MPa}$ ). The headed bars evaluated in this paper represent a variety of manufacturing processes, as illustrated for No. 8 (No. 25) bars in Fig. 1: friction-forged, taper-threaded, and cold-swaged, some of the latter incorporating coupling sleeves. All the heads meet the requirements for Class HA heads in ASTM A970-18 and most of them conform to the requirements for Class HA heads in ASTM A970-13a (heads permitted by ACI 318-14). Three versions of the "cold-swaged threaded coupling sleeve headed bar," which met the requirements of ASTM A970-13a but did not satisfy the requirements of an HA head because the coupling sleeve exceeded the limit on the size of the obstruction in that specification, are described as "large obstruction" heads in this paper. Details of heads are provided in Appendix A."

"The Appendix is available at www.concrete.org/publications in PDF format, appended to the online version of the published paper. It is also available in hard copy from ACI headquarters for a fee equal to the cost of reproduction plus handling at the time of the request.

\section{Test specimens}

The test specimens were designed to simulate an exterior beam-column joint, as shown in Fig. 2. The headed bars embedded in the column represented top longitudinal reinforcement of the beam and the bearing member below the headed bars served as the compression zone of the virtual beam. Details of specimens are provided in Appendix A. The embedment lengths $\ell_{e h}$ were chosen so that the specimens failed in anchorage (governed by concrete failure) rather than fracture of the headed bar. The embedment length $\ell_{e h}$ is defined as the distance from the bearing face of the head to the column front face (Fig. 2). Initially, the embedment length was chosen to be $80 \%$ of the development length that was required in Section 25.4.4.2 in ACI 318-14 and, later, as more specimens were tested, it was calculated based on results from previous test results. The depth of the column $h$ thus equaled the sum of the embedment length, thickness of the head, and cover to the back of the head. The width of the column $w$ was determined by the out-to-out spacing between 


\begin{tabular}{|c|c|c|c|c|c|}
\hline \multirow[b]{2}{*}{ Material } & \multicolumn{4}{|c|}{ Quantity (SSD) } & \multirow[b]{2}{*}{$\begin{array}{c}\text { SG or } \\
\text { BSG (SSD) }\end{array}$} \\
\hline & $\begin{array}{c}5000 \mathrm{psi} \\
w / c=0.44\end{array}$ & $\begin{array}{c}8000 \text { psi } \\
w / c=0.32\end{array}$ & $\begin{array}{l}12,000 \mathrm{psi} \\
w / c=0.29\end{array}$ & $\begin{array}{c}15,000 \mathrm{psi} \\
w / \mathrm{cm}=0.23\end{array}$ & \\
\hline Type I/II cement, lb/yd ${ }^{3}$ & 600 & 700 & 750 & 760 & 3.2 \\
\hline Type C fly ash, lb/yd ${ }^{3}$ & - & - & - & 160 & 2.3 \\
\hline Silica fume, $\mathrm{lb} / \mathrm{yd}^{3}$ & - & - & - & 100 & 2.2 \\
\hline Water, $1 \mathrm{~b} / \mathrm{yd}^{3}$ & 263 & 225 & 217 & 233 & 1.0 \\
\hline Kansas river sand, $\mathrm{lb} / \mathrm{yd}^{3}$ & 1396 & 1375 & 1050 & 1138 & 2.63 \\
\hline Pea gravel, lb/yd $\mathrm{d}^{3}$ & - & - & 316 & - & 2.60 \\
\hline Crushed limestone, $\mathrm{lb} / \mathrm{yd}^{3}$ & $1735^{*}$ & 1683 & 1796 & - & 2.59 \\
\hline Granite, $1 \mathrm{~b} / \mathrm{yd}^{3}$ & - & - & - & 1693 & 2.61 \\
\hline Water reducer, oz (U.S.) & $30^{\dagger}$ & $171^{\dagger}$ & $104^{+}$ & $205^{\ddagger}$ & - \\
\hline
\end{tabular}

* Granite used in place of limestone for Group 2 - refer to Table A2 in Appendix A.

Mid- to high-range polycarboxylate-based water reducer.

${ }^{\star}$ High-range polycarboxylate-based water reducer.

Notes: SG is specific gravity; BSG (SSD) is bulk specific gravity (

the headed bars and the side cover to the bar. For most of the specimens, the headed bars were anchored near the far side of the column with the back of the head touching the column longitudinal bars. For some specimens, the headed bars were anchored in the middle of the column, as shown in Fig. 2(d). The nominal value of cover to the back of the head in these specimens ranged from 3 to 7 in. ( 75 to $175 \mathrm{~mm}$ ). The height of the column was chosen to prevent interference of the force from the upper compression member of the test frame (Fig. 2) with the forces within the joint. A column height of 96 in. $(2440 \mathrm{~mm})$ was used for specimens containing No. 11 (No. 36) headed bars and, with one exception, 54 in. (1370 $\mathrm{mm}$ ) for the specimens containing No. 5 or No. 8 (No. 16 or No. 25) headed bars. One test group of No. 8 (No. 25) headed bars had a column height of 96 in. $(2440 \mathrm{~mm})$.

The longitudinal and transverse reinforcement outside the joint region for the columns were designed assuming that the column was simply supported with supports at the upper compression member and the bearing member (Fig. 2) and that all headed bars in the joint would reach their expected failure stress simultaneously. Shear capacity within the joint region was provided by the concrete and, if used, the confining reinforcement (ties) in the form of hoops within the joint.

For a small number of the specimens containing three headed bars, hairpin reinforcement was used to hold the middle headed bar in place. Two sets, each containing two No. 3 (No. 10) bars, were placed perpendicular to the headed bars, spaced at 3 in. $(76 \mathrm{~mm}$ ) along the embedded bars, as shown in Fig. A5 of Appendix A. The specimens containing hairpin reinforcement are identified with $\mathrm{HP}$ at the end of the specimen designation (Appendix A).

Some specimens had strain gauges installed to monitor the change in strain in the headed bars and in the confining reinforcement within the joint region. Figure 2(e) shows the locations of the strain gauges. For each specimen, strain gauges were attached to at least one headed bar and to legs parallel to the headed bars for all hoops in the joint region. If a specimen had three or four headed bars, a strain gauge was also attached to a middle bar. Two strain gauges were mounted on the headed bars, with one gauge placed $1.5 \mathrm{in}$. $(38 \mathrm{~mm})$ from the head bearing face and the other gauge placed $1 \mathrm{in} .(25 \mathrm{~mm})$ from the front face of the column. The strain gauges on the hoops were placed at the middle of a leg parallel with the headed bars. All gauges were placed on the same side of the column.

\section{Material properties}

Non-air-entrained ready mixed concrete with nominal strengths of $5000,8000,12,000$, and 15,000 psi $(35,55,83$, and $103 \mathrm{MPa}$ ) was used in this study, with the exception of one group of No. 11 (No. 36) headed-bar beam-column joint specimens that contained air-entrained concrete. A mid- to high-range polycarboxylate-based water reducer was used as a water-reducing agent for the 5000 and 8000 psi (35 and $55 \mathrm{MPa}$ ) concrete mixtures, and a highrange polycarboxylate-based water reducer was used for the 12,000 and 15,000 psi ( 83 and $103 \mathrm{MPa}$ ) concrete mixtures. The mixture proportions are given in Table 1 . The maximum aggregate size was $3 / 4$ in. (19 mm).

The headed bars were made of ASTM A1035 Grade 120 (830 MPa) steel to help ensure that anchorage capacity was governed by the surrounding concrete and confining reinforcement and not the tensile strength of the headed bars. The confining reinforcement consisted of either No. 3 or No. 4 (No. 10 or No. 13) ASTM A615 Grade 60 (420 MPa) bars. Most column longitudinal reinforcement was fabricated from ASTM A615 Grade 60 (420 MPa) steel; in some specimens with a high flexural demand, ASTM A615 Grade 80 $(550 \mathrm{MPa})$ or ASTM A1035 Grade $120(830 \mathrm{MPa})$ steel was used.

The physical properties of the headed bars are shown in Table 2 . The headed bars are designated by a letter based on the type of head and a number representing the bearing area as a multiple of bar area. The friction-forged, taperthreaded, and cold-swaged headed bars are identified by F, $\mathrm{T}$, and S, respectively; the cold-swaged threaded coupling sleeve headed bars are represented by $\mathrm{O}$ in recognition 
Table 2-Headed bar physical properties

\begin{tabular}{|c|c|c|c|c|c|c|c|c|}
\hline \multirow[b]{2}{*}{ Bar size } & \multirow[b]{2}{*}{ Heads } & \multirow{2}{*}{$\begin{array}{l}\text { Yield strength, } \\
\text { ksi }\end{array}$} & \multirow{2}{*}{$\begin{array}{c}\text { Nominal } \\
\text { diameter, in. }\end{array}$} & \multirow{2}{*}{$\begin{array}{l}\text { Average rib } \\
\text { spacing, in. }\end{array}$} & \multicolumn{2}{|c|}{ Average rib height } & \multirow{2}{*}{$\begin{array}{l}\text { Average gap } \\
\text { width, in. }\end{array}$} & \multirow[b]{2}{*}{ Relative rib area } \\
\hline & & & & & $A^{*}$, in. & $\mathrm{B}^{\dagger}$, in. & & \\
\hline No. 5 & F4.0, F13.1 & 139.0 & 0.625 & 0.423 & 0.037 & 0.035 & 0.319 & 0.07 \\
\hline No. 8 & F4.1, F9.1 & 129.0 & 1 & 0.633 & 0.065 & 0.060 & 0.347 & 0.084 \\
\hline No. 8 & T4.0, T9.5 & 120.0 & 1 & 0.590 & 0.067 & 0.062 & 0.287 & 0.095 \\
\hline No. 8 & $\begin{array}{l}\text { S6.5, S9.5, S14.9, } \\
\text { O4.5, O9.1, O12.9 }\end{array}$ & 115.9 & 1 & 0.580 & 0.069 & 0.063 & 0.280 & 0.099 \\
\hline No. 11 & F3.8, F8.6, S5.5, O4.5 & 135.0 & 1.41 & 0.838 & 0.097 & 0.092 & 0.394 & 0.099 \\
\hline
\end{tabular}

*Per ASTM A615, A706.

Per ACI 408R-03.

Notes: No. 5 (No. 16) No. 8 (No. 25), No. 11 (No. 36); 1 in. $=25.4$ mm; 1 psi $=0.006895$ MPa.

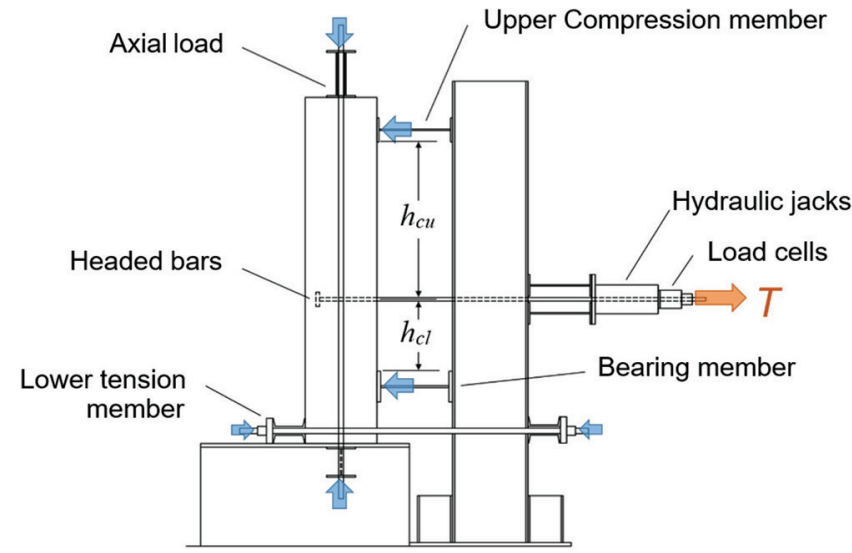

Fig. 3-Testing frame and forces applied to specimens during testing.

of the sleeves serving as large obstructions. The coupling sleeve contained a tapered section (gap) adjacent to the head followed by a larger obstruction (refer to Fig. A2 in Appendix A). The width of the gap in these headed bars was $3 / 8$ in. $(9.5 \mathrm{~mm})$ for No. 5 and No. 8 (No. 16 and No. 25 ) bars, and $5 / 8$ in. $(16 \mathrm{~mm}$ ) for No. 11 (No. 36 ) bars, and the depth of the gap was less than the width. The upper limit on the diameter and length of the obstruction measured from the bearing face of the head was $2.5 d_{b}$ and $5.25 d_{b}$, respectively. The friction-forged headed bars contained small obstructions complying with the requirements for Class HA heads specified in ASTM A970-13a. The obstructions in these heads had an upper limit on diameter of $1.5 d_{b}$ and an upper limit on length of $0.6 d_{b}$ for No. 8 and No. 11 bars and $0.75 d_{b}$ for No. 5 bars. The taper threaded and cold-swaged headed bars had no obstructions. Details of the heads are provided in Appendix A.

\section{Test procedure}

The test frame was a modified version of the apparatus used by Marques and Jirsa (1975). Figure 3 shows the applied loads and reactions on a specimen. As shown in the figure, the upper compression member, placed at the top of the specimen, and the lower tension member, placed at the bottom of the specimen, were used to prevent overturning of the specimen. The bearing member, placed below the headed bars, simulated the beam compression region on the front face of the column. The widths of the upper compres-
Table 3-Position of bearing members

\begin{tabular}{c|c|c|c}
\hline Headed bar size & $\begin{array}{c}\text { No. 5 } \\
\text { No. 16) }\end{array}$ & $\begin{array}{c}\text { No. 8 } \\
(\text { No. 25) }\end{array}$ & $\begin{array}{c}\text { No. 11 } \\
\text { (No. 36) }\end{array}$ \\
\hline $\begin{array}{c}\text { Specimen height } \\
\text { Distance from center of headed bar } \\
\text { to top edge of lower compression } \\
\text { plate, } h_{c l}{ }^{*}\end{array}$ & 54 in. & 54 in. & $96 \mathrm{in.}$ \\
\hline $\begin{array}{c}\text { Distance from center of headed bar } \\
\text { to bottom edge of upper compression } \\
\text { plate, } h_{c u}{ }^{*}\end{array}$ & 18.25 in. & 18.25 in. & 44.25 in. \\
\hline \begin{tabular}{c} 
pln. \\
\hline
\end{tabular} & & & \\
\hline
\end{tabular}

${ }^{*}$ Refer to Fig. 3

Note: 1 in. $=25.4 \mathrm{~mm}$.

sion member and bearing member were $6-5 / 8$ in. $(168 \mathrm{~mm})$ and $8-3 / 8$ in. $(213 \mathrm{~mm})$, respectively. The positions of the bearing members were adjustable; the dimensions are shown in Table 3. One group of No. 8 (No. 25) headed-bar specimens was tested with a distance between the headed bar and top of the compression plate $\left(h_{c l}\right)$ of $20 \mathrm{in} .(508 \mathrm{~mm})$ (as opposed to the standard $10.25 \mathrm{in}$. [260 mm]) to simulate a deep beam-column joint. These specimens are identified with "DB" at the end of the specimen designation.

Before loading the headed bars, an axial compressive load was applied to the column. For specimens that were 96 in. $(2438 \mathrm{~mm}$ ) high (all No. 11 [No. 36] headed-bar specimens and one group of No. 8 [No. 25] headed-bar specimens), an axial load providing a stress of $280 \mathrm{psi}(1.9 \mathrm{MPa})$ was applied; for specimens that were $54 \mathrm{in}$. (1372 mm) high (all No. 5 [No. 16] headed-bar specimens and all but one group of No. 8 [No. 25] headed-bar specimens), an axial load of approximately $30 \mathrm{kip}(133.4 \mathrm{kN})$ was applied (corresponding to a range in axial stress of 93 to 243 psi [0.64 to $1.7 \mathrm{MPa}$ ]). This axial loading level was assumed to have no significant influence on the anchorage strength of headed bars based on the observation by Marques and Jirsa (1975) that changes in axial load (with an axial stress of up to $3080 \mathrm{psi}$ [21.2 MPa]) did not have a significant effect on the anchorage strength of beam-column joint specimens containing hooked bars. Tensile loads were applied monotonically to the headed bars using hydraulic jacks in increments of 5, 10, 15, or 20 kip $(22.2,44.5,66.7$, or $89 \mathrm{kN})$ per specimen, depending on the estimated failure load of the specimen. Loading was paused after each increment to allow cracks to be marked on the specimen. When a specimen approached its estimated failure load 
or had an obvious sign of failure (such as continuous bar slip or the load dropping during an interval), the specimen was then loaded to failure without marking additional cracks. After failure, loose concrete was removed from the specimen to expose internal cracks. Tests lasted approximately 30 minutes.

\section{Cracking patterns}

\section{TEST RESULTS}

Although cracking differed in terms of quantity and shape in different specimens, overall crack propagation followed similar patterns. First, a horizontal crack initiated on the front face of the column at the level of the headed bars, extending slightly toward both sides of the column (Fig. 4(a)). As the load increased, the horizontal cracks on the column front face connected between the bars, occasionally accompanied by small radial cracks extending from the bars. At the same time, the horizontal crack on the side of the column continued to grow toward the position of the head, with diagonal cracks branching from the horizontal crack toward the front face (Fig. 4(b)). With increasing load, more horizontal cracks due to column flexure began to appear on the front face below and/or above the level of the headed bars, extending slightly around the side of the column. On the side of the column, a large diagonal crack occurred within the joint region, extending from the position of the head toward the bearing member (the compression region of the virtual beam); the crack above the joint region extended diagonally toward the upper compression member or upward toward the top of the column (Fig. 4(c)). As load further increased, the existing cracks on the side became wider and continued growing toward the front face of the column, with new cracks branching from the existing cracks on both side and front faces (Fig. 4(d)). The amount of cracking was directly related to the amount of confining reinforcement within the joint region; specimens with confining reinforcement generally exhibited more cracks prior to failure than those without confining reinforcement.

The cracking patterns for deep-beam specimens (which had relatively short embedment lengths compared to the distance from the center of the headed bar to the top of the bearing member) were slightly different from those of the conventional specimens shown in Fig. 4, with a large diagonal crack extending at an angle of about 35 degrees with respect to the direction of the column longitudinal bars, from the bearing head toward the top of the bearing member for specimens both without and with confining reinforcement. For the conventional specimens, the large diagonal crack extended between the bearing head and the top of the bearing member, with an angle usually flatter than the large diagonal crack observed in deep-beam specimens (that is, greater than 35 degrees with respect to the direction of column longitudinal bars).

\section{Failure modes}

Of the 202 beam-column joint specimens tested, 196 exhibited an anchorage failure, as designed. An anchorage failure is defined as failure of the concrete around the head, accompanied by slip and loss of capacity of the headed bar. Two modes of failure were observed: concrete breakout

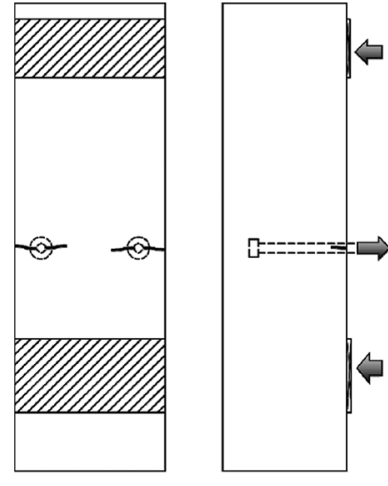

(a)
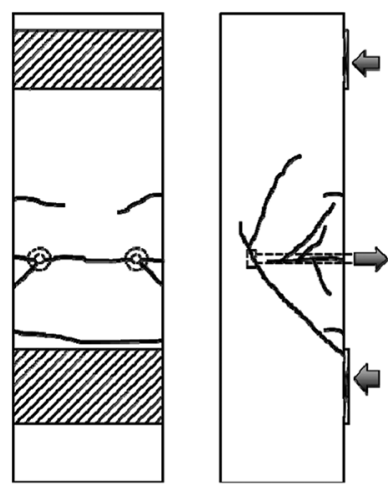

(c)
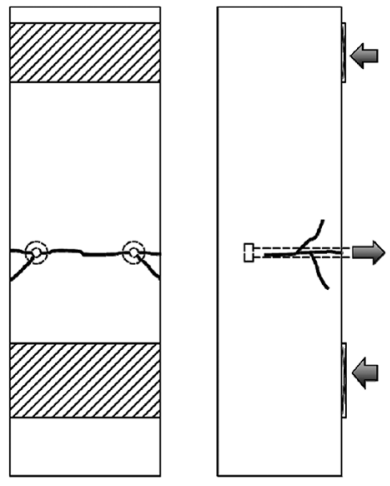

(b)
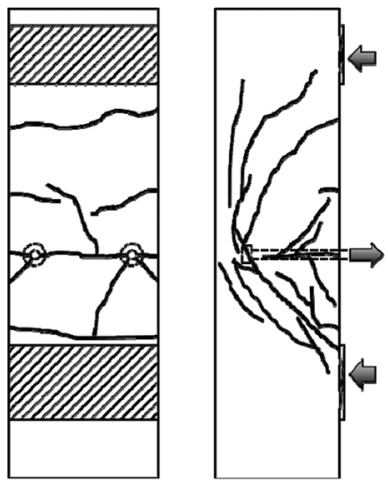

(d)
Fig. 4-Front and side views of specimens illustrating crack progression from: (a) low loads to (d) failure.

and side-face blowout. Headed bars with large obstructions $(\mathrm{O} 4.5, \mathrm{O} 9.1$, and O12.9) that did not meet the requirement in ASTM A970-13a for HA heads exhibited failure modes that were similar to those with HA heads. All deep-beam specimens exhibited a concrete breakout failure.

Six specimens were not loaded to failure because of safety concerns; either the bar was loaded to near its fracture strength or the testing equipment had reached its capacity. In this study, "bar yielding" is used to describe the specimens that did not fail in anchorage, although some specimens with anchorage failure had bars loaded beyond the nominal yield strength (120 ksi [830 MPa]).

Concrete breakout was observed in the majority (149) of the specimens. It occurred when concrete in front of the head was pulled out and separated from the specimen. Two types of failure surface were observed: the first was coneshaped, as shown in Fig. 5(a); the second type was similar to the first within the joint region, but above the joint region, a crack extended from the head toward the top of the column, as shown in Fig. 5(b), which resulted in various degrees of splitting of the column back cover. Occasionally, the longitudinal splitting crack (Fig. 5(b)) did not appear until the joint was near failure; in these instances, the longitudinal crack appeared suddenly and grew rapidly. It was, however, more common for the longitudinal crack to propagate slowly throughout the test.

Table 4 summarizes the number of specimens exhibiting each of the two types of breakout failure. Specimens exhibiting side-face blowout failure are not included in the table. 


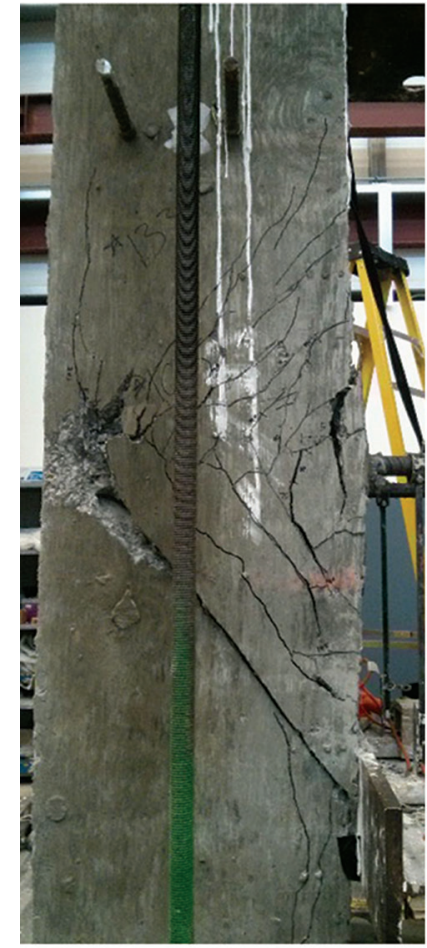

(a)

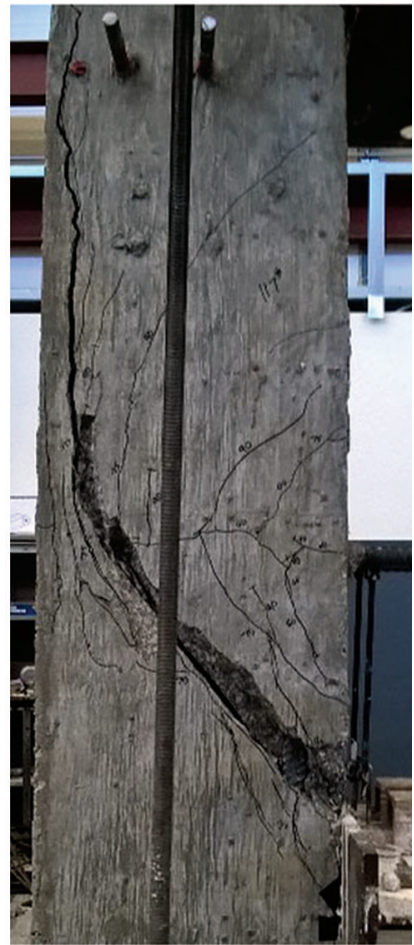

(b)
Fig. 5-Two types of breakout failure: (a) cone-shaped; and (b) back cover splitting.

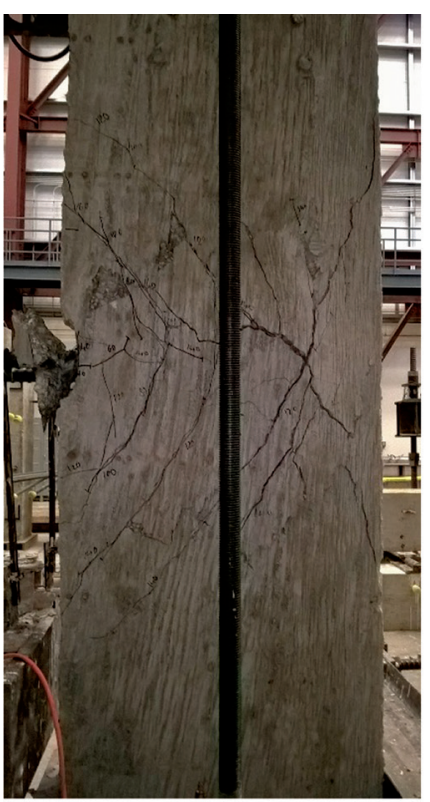

(a)

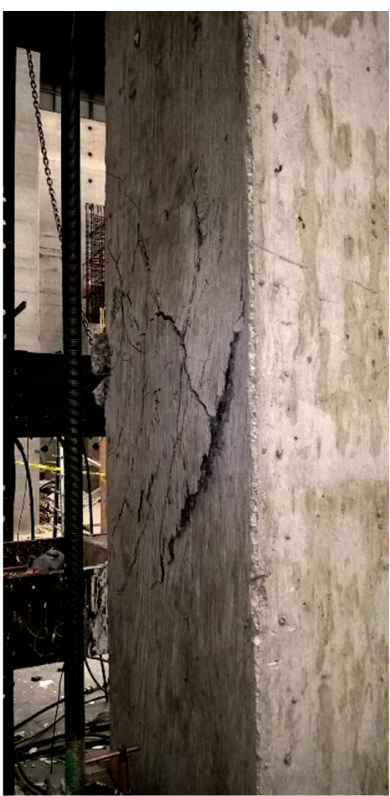

(b)
Fig. 6-Side-face blowout: (a) side view; and (b) back view.

If a specimen with breakout failure exhibited both types of cracking above the joint region, the maximum crack width was used to determine the category of the failure surface. The table is based on the available record for the test specimens. The failure type of a specimen (breakout failure or side-face blowout) was recorded at the time of the test, while the type of breakout failure (cone-shaped failure surface or back cover splitting) was determined after the test based on photographs taken during the tests. One early specimen that
Table 4-Summary of specimens with different types of breakout failure

\begin{tabular}{|c|c|c|c|}
\hline \multicolumn{2}{|c|}{ Specimen details } & \multirow[b]{2}{*}{$\begin{array}{l}\text { Number with } \\
\text { cone-shaped } \\
\text { failure surface }\end{array}$} & \multirow[b]{2}{*}{$\begin{array}{c}\text { Number with } \\
\text { back cover } \\
\text { splitting }\end{array}$} \\
\hline $\begin{array}{c}\text { Confining } \\
\text { reinforcement } \\
\text { level }\end{array}$ & Spacing* & & \\
\hline \multirow{2}{*}{$\begin{array}{l}\text { Without } \\
\text { confining } \\
\text { reinforcement }\end{array}$} & Closely spaced & 12 & 29 \\
\hline & Widely spaced & 21 & 12 \\
\hline \multirow{2}{*}{$\begin{array}{l}\text { With confining } \\
\text { reinforcement }\end{array}$} & Closely spaced & 17 & 21 \\
\hline & Widely spaced & 25 & 11 \\
\hline \multicolumn{2}{|c|}{ Total } & 75 & 73 \\
\hline
\end{tabular}

"“Closely spaced" refers to specimens with a center-to-center bar spacing less than $8 d_{b}$; "widely spaced" refers to specimens with a center-to-center bar spacing greater than or equal to $8 d_{b}$.

was recorded as breakout failure but lacked relevant photo records is not included in Table 4.

As shown in Table 4, approximately half ( 75 out of 148 ) of the specimens exhibiting a breakout failure had a coneshaped failure surface. Cone-shaped failure surfaces were more likely to occur in specimens with widely spaced bars, while back cover splitting tended to occur in specimens with closely spaced bars and no confining reinforcement within the joint region. A closer look into the test data reveals no direct relationship between the shape of the failure surface and the anchorage strength of headed bars exhibiting a concrete breakout failure.

Of the 196 specimens that had an anchorage failure, 47 exhibited side-face blowout. Side-face blowout occurred when the movement of the head resulted in local damage to the side cover around the head, as shown in Fig. 6. The damage was often characterized by separation of the cracked concrete cover.

Some specimens with a concrete breakout failure also exhibited damage on the side, especially along the dominant crack within the joint region. In such cases, however, failure was characterized as side-face blowout only if the failure appeared to be the direct result of local damage of the side cover around the head. Specimens with side-face blowout did not exhibit splitting cracks above the joint region.

A secondary failure often occurred in conjunction with a concrete breakout or side-face blowout failure. Secondary failures tended to be one of two types-local front breakout or back cover spalling. Local front breakout (Fig. 7(a)) was characterized by a small portion of concrete cover near the bar being pulled out of the column front face. Back cover spalling occurred when the splitting crack above the joint region (Fig. 7(b)) widened, with the concrete cover at the back of the column separating from the column.

\section{Comparison of test results with $\mathrm{ACI}$ 318-14}

The average bar force at failure, $T$, defined as the peak force applied to the specimen divided by the number of headed bars, is presented in Appendix A, along with the maximum bar force measured for each bar. The average bar force at failure, rather than the maximum load per bar, is treated as the failure load. 


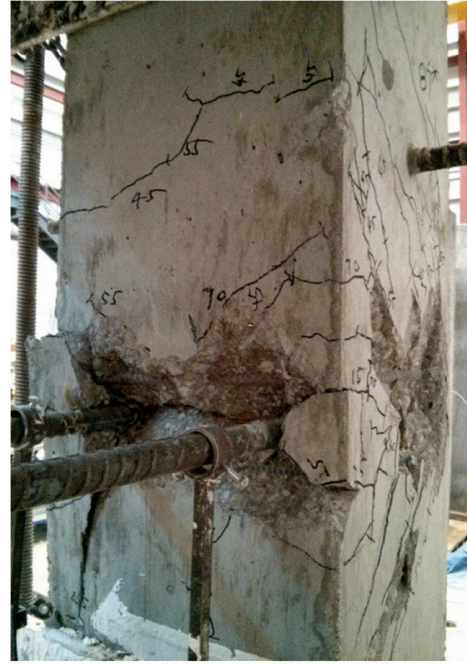

(a)

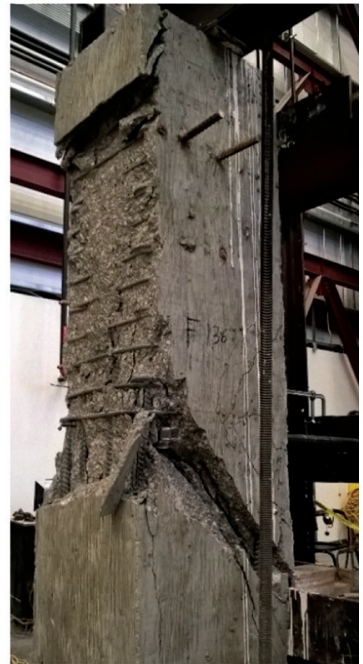

(b)
Fig. 7-Secondary failure types: (a) local front breakout; and (b) back cover spalling.

A regression analysis technique based on dummy variables (Draper and Smith 1981) was used to identify trends in the data. Dummy variables analysis is a least-squares regression analysis method that allows differences in populations to be taken into account when formulating relationships between principal variables. For example, the effect of embedment length $\ell_{e h}$ on the bar force at failure $T$ can be found for different bar sizes based on the assumption that the effect of changes in $\ell_{e h}$ on changes in $T$ (slope of the regression line) is the same for all the bar sizes considered, but that the absolute value of $T$ for a given $\ell_{e h}$ differs for each bar size, resulting in different intercepts for the individual regression lines. ${ }^{\dagger}$

ACI provisions - In accordance with Section 25.4.4.2(a) of ACI 318-14, the development length of a headed bar $\ell_{d t}$ (in. or $\mathrm{mm}$ ) is given by

$$
\begin{gathered}
\ell_{d t}=\left(\frac{0.016 f_{y} \psi_{e}}{\sqrt{f_{c}^{\prime}}}\right) d_{b}(\mathrm{in} . \mathrm{lb}) \\
\ell_{d t}=\left(\frac{0.19 f_{y} \psi_{e}}{\sqrt{f_{c}^{\prime}}}\right) d_{b}(\mathrm{SI})
\end{gathered}
$$

where $f_{y}$ is the yield strength of the bar (psi or MPa); $f_{c}^{\prime}$ is the compressive strength of concrete (psi or MPa); $d_{b}$ is bar diameter (in. or $\mathrm{mm}$ ); and $\psi_{e}=1.2$ modification factor for epoxy-coated or zinc and epoxy dual-coated bars and $=1.0$ for uncoated bars. The values of $f_{y}$ and $f_{c}{ }^{\prime}$ used to calculate $\ell_{d t}$ have upper limits in the calculation of 60,000 and $6000 \mathrm{psi}(420$ and $40 \mathrm{MPa}$ ), respectively. The development length $\ell_{d t}$ represents the minimum embedment length required to develop the yield strength of the bar and may be no less than the larger of $8 d_{b}$ or 6 in. $(150 \mathrm{~mm})$.

To evaluate the test results, Eq. (1) is solved for $f_{y}$, and $f_{y}$ is replaced by $f_{s, \mathrm{ACI}}, \ell_{d t}$ by $\ell_{e h}$, and $f_{c}^{\prime}$ by $f_{c m}$ (measured concrete

This explanation of dummy variables analysis taken from Sperry et al. (2017). compressive strength). The result, setting $\psi_{e}=1$ (all headed bars in this study were uncoated) is given in Eq. (2)

$$
\begin{gathered}
f_{s, A C I}=\frac{\ell_{e h} \sqrt{f_{c m}}}{0.016 d_{b}} \text { (in.-lb) } \\
f_{s, A C I}=\frac{\ell_{e h} \sqrt{f_{c m}}}{0.19 d_{b}} \text { (SI) }
\end{gathered}
$$

Comparisons - The ratio of test to calculated bar stress at failure, $f_{s u} / f_{s, A C I}$, is used to compare the test results from beam-column joint specimens with the ACI equation. $f_{s u}$ is taken as the total peak load applied on a specimen divided by the total area of the headed bars. $f_{s, A C I}$ is calculated using Eq. (2), in which $\ell_{e h}$ is the average measured embedment length (corresponding to $\ell_{\text {eh,avg }}$ in Table A.1 of Appendix A), and $f_{c m}$ is the measured concrete compressive strength. The $6000 \mathrm{psi}(40 \mathrm{MPa})$ limit on $f_{c m}$ is not used in the comparisons that follow to: 1) determine the degree to which the 0.5 power accurately characterizes the influence of concrete compressive strength on anchorage strength across all concrete strengths; and 2) determine if the limit can be raised or eliminated.

For the comparisons, test results are grouped based on bar size and head size. In some cases, heads with similar but not identical bearing areas are combined to obtain a sufficient number of data points for analysis. For example, the results for the two specimens with No. 8 (No. 25) bars with $12.9 A_{b}$ (bearing area $=12.9 A_{b}$ ) heads and the five specimens with No. 8 (No. 25) bars with $14.9 A_{b}$ heads are combined. With the exception of the example given above, combined heads differed in area by less than $1 A_{b}$.

The ratio of bar stress at failure to bar stress calculated using Eq. (2), $f_{s u} / f_{s, A C I}$, is plotted versus the measured concrete compressive strength $f_{c m}$ in Fig. 8 through 13. Each data point in the plot represents an individual specimen.

Widely spaced bars-Figures 8 through 10 compare $f_{s u} / f_{s, A C I}$ to $f_{c m}$ for the specimens in this study with two widely spaced headed bars. Figure 8 includes 46 specimens without confining reinforcement, Fig. 9 includes 18 specimens with two No. 3 (No. 10) hoops as confining reinforcement, and Fig. 10 includes 35 specimens with No. 3 (No. 10) hoops spaced at $3 d_{b}$. The trend lines in the figures are obtained using dummy variables analysis. The order of the lines from highest to lowest is listed in the legend.

The trend lines in the three figures have negative slopes, indicating that the effect of concrete compressive strength is overestimated by the 0.5 power in the ACI 318 equation. The values of $f_{s u} / f_{S, A C I}$ are greater than 1.0, with the exception of two specimens containing No. 11 (No. 36) bars, one without confining reinforcement (Fig. 8) and the other with two No. 3 (No. 10) hoops as confining reinforcement (Fig. 9). This indicates that the ACI 318 equation is generally conservative. The ACI 318 equation is overconservative for No. 5 (No. 16) bars and becomes less so as bar size increases.

The order of the trend lines also reflects the effect of head size. For the No. 5 (No. 16) bars at all levels of confinement, the $f_{s u} / f_{s, A C I}$ values for the $13.1 A_{b}$ heads are consistently 


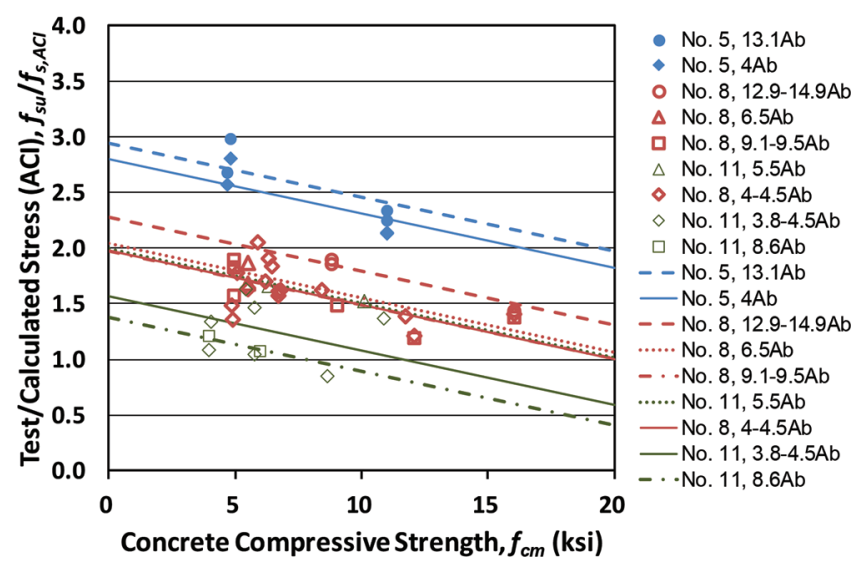

Fig. 8-Ratio of test-to-calculated stress $\mathrm{f}_{\mathrm{su}} / \mathrm{f}_{\mathrm{s}, \mathrm{ACI}}$ versus measured concrete compressive strength $\mathrm{f}_{\mathrm{cm}}$ for specimens without confining reinforcement in joint region.

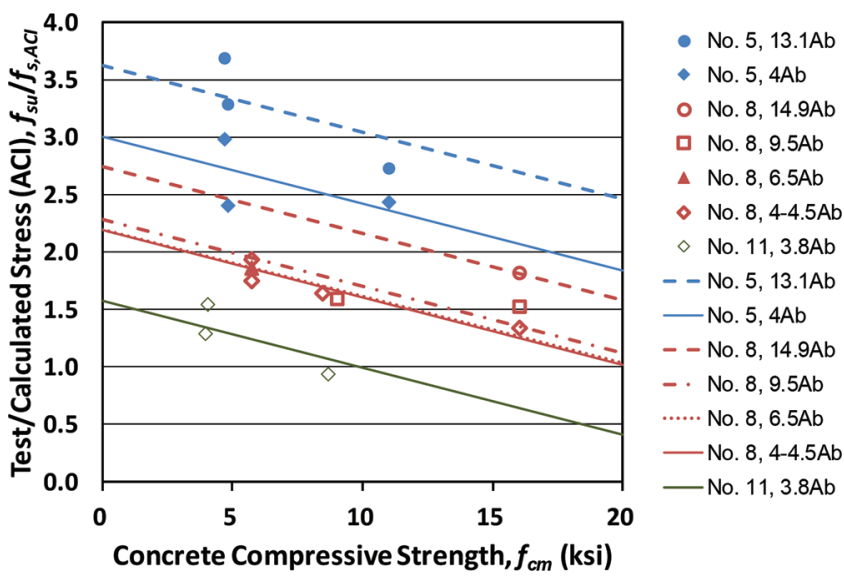

Fig. 9-Ratio of test-to-calculated stress $\mathrm{f}_{\mathrm{su}} / \mathrm{f}_{\mathrm{s}, \mathrm{ACI}}$ versus measured concrete compressive strength $\mathrm{f}_{\mathrm{cm}}$ for specimens with two No. 3 hoops in joint region.

greater than those for the $4 A_{b}$ heads. For the No. 8 (No. 25) bars, the $f_{s u} / f_{s, A C I}$ values for 12.9 to $14.9 A_{b}$ heads are far greater than those for the smaller heads for specimens without confining reinforcement or with two No. 3 (No. 10) hoops as confinement. For the No. 8 (No. 25) bars with No. 3 (No. 10) hoops spaced at $3 d_{b}$, the $f_{s u} / f_{s, A C I}$ values are similar for $9 A_{b}$ heads and larger, whereas heads with 4 to $6.5 A_{b}$ bearing area exhibit lower values. While a trend is observed for No. 5 and No. 8 bars that larger heads tend to give in greater $f_{s u} / f_{s, A C I}$ values, the No. 11 (No. 36) bar specimens do not show this trend for heads with bearing areas ranging from 3.8 to $8.6 A_{b}$. In addition to bar and head size, Fig. 8 through 10 show that the presence of confining reinforcement within the joint region results in an increase in $f_{s u} / f_{s, \mathrm{ACI}}$. This indicates that confining reinforcement improves the anchorage strength of headed bars, a factor that is not accounted for in the provisions in ACI 318-14 for the development of headed bars.

Closely spaced bars-Figures 11 through 13 compare the ratio $f_{s u} / f_{s, A C I}$ with the center-to-center bar spacing for specimens with three or four closely spaced headed bars and specimens with two widely spaced headed bars with the centerto-center bar spacing. Specimens with a center-to-center bar

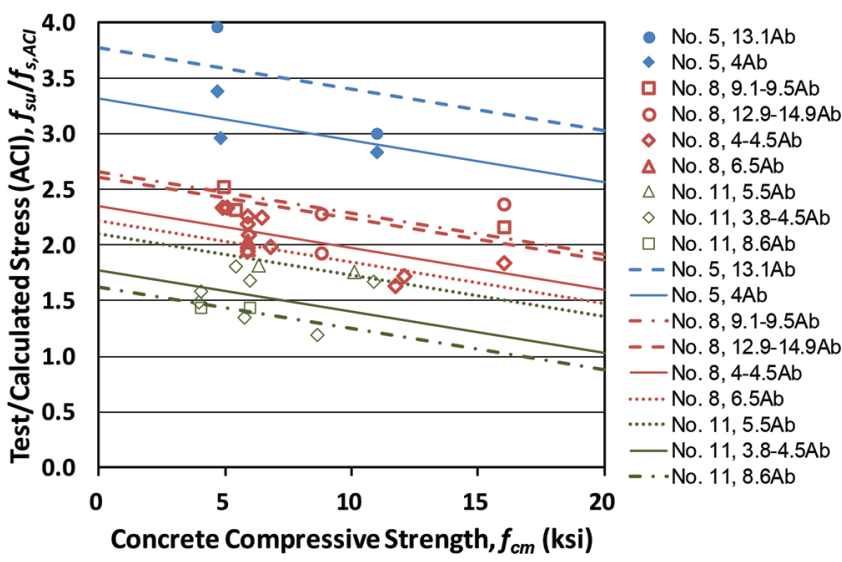

Fig. 10-Ratio of test-to-calculated stress $\mathrm{f}_{\mathrm{su}} / \mathrm{f}_{\mathrm{s}, \mathrm{ACI}}$ versus measured concrete compressive strength $\mathrm{f}_{\mathrm{cm}}$ for specimens with No. 3 hoops spaced at $3 \mathrm{~d}_{\mathrm{b}}$ in joint region.

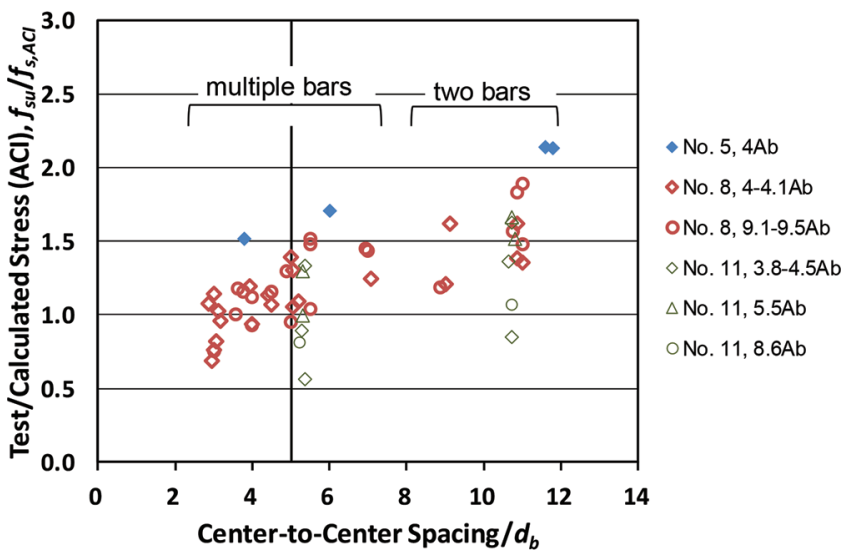

Fig. 11-Ratio of test-to-calculated stress $\mathrm{f}_{\mathrm{su}} / \mathrm{f}_{\mathrm{s}, \mathrm{ACI}}$ versus center-to-center spacing for headed bars without confining reinforcement in joint region.

spacing less than $5 d_{b}\left(5 d_{b}\right.$ is indicated by the vertical lines in Fig. 11 to 13 ) did not meet the minimum $4 d_{b}$ clear spacing requirement in ACI 318 (although it should be noted that a $3 d_{b}$ clear spacing is permitted for use in beam-column joints of special moment frames in seismic-resisting systems). Similar to Fig. 8 through 10, Fig. 11 through 13 represent results for specimens with different amounts of confining reinforcement in the joint region.

The three figures show that, relative to the current ACI Code provisions, closely spaced headed bars generally have lower anchorage strengths than widely spaced bars. The overall upward trend of $f_{s u} / f_{s, A C I}$ as bar spacing increases indicates that bar spacing has an effect on anchorage strength that the current Code provisions do not account for. Most headed bars with less than $5 d_{b}$ center-to-center bar spacing (below the ACI $4 d_{b}$ clear spacing requirement) had $f_{s u} / f_{s, A C I}$ values greater than 1 ; of the specimens with $f_{s u} / f_{s, A C I}$ values less than 1, all but one had no confining reinforcement within the joint region. Therefore, it appears that the current limit on bar spacing could be safely reduced to allow for use of more closely spaced headed bars, provided that confining reinforcement is used in the joint.

For both widely spaced and closely spaced bars, the ACI Code provisions are most conservative for No. 5 bars, 


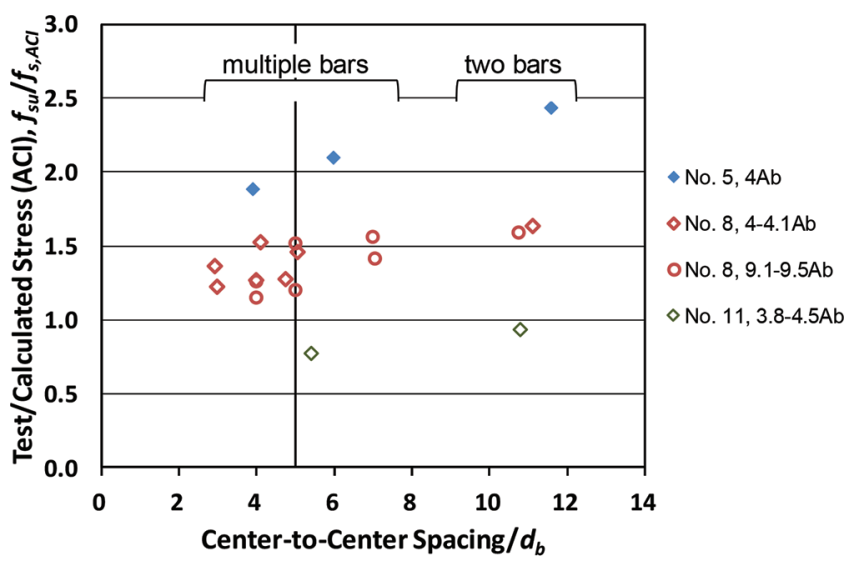

Fig. 12-Ratio of test-to-calculated stress $\mathrm{f}_{\mathrm{su}} / \mathrm{f}_{\mathrm{s}, \mathrm{ACI}}$ versus center-to-center spacing for headed bars with two No. 3 hoops in joint region.

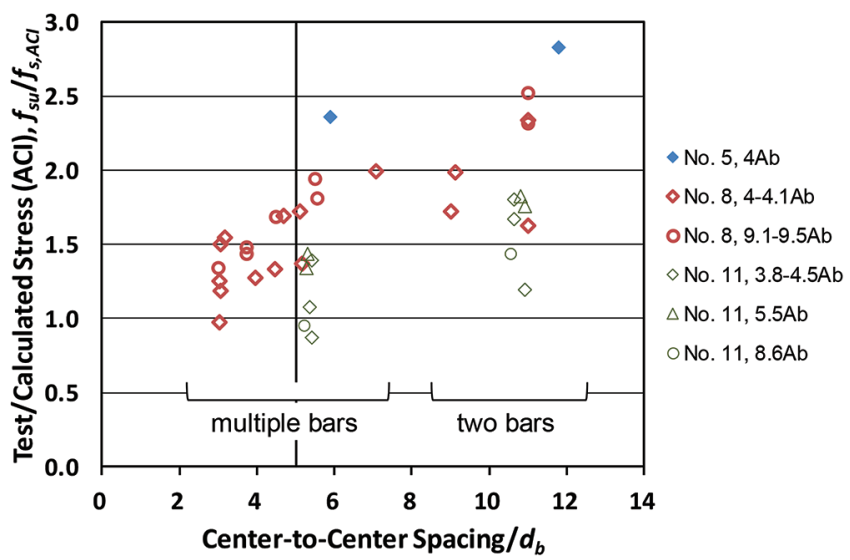

Fig. 13-Ratio of test-to-calculated stress $\mathrm{f}_{\mathrm{su}} / \mathrm{f}_{\mathrm{s}, \mathrm{ACI}}$ versus center-to-center spacing for headed bars with No. 3 hoops spaced at $3 \mathrm{~d}_{\mathrm{b}}$ in joint region.

becoming less conservative as the bar size increases. Of the 10 specimens that had at least $5 d_{b}$ center-to-center bar spacing (meeting the ACI requirement) and $f_{s u} / f_{s, A C I}$ values less than 1, nine specimens contained No. 11 bars and one specimen contained No. 8 bars.

The comparisons with ACI 318-14 reveal that the current code provisions for headed bar development do not accurately capture the effects of concrete compressive strength, bar spacing, or bar size on the anchorage strength of headed bars and should be adjusted to reflect the effects of key parameters over the broader range of values currently used in construction.

\section{SUMMARY AND CONCLUSIONS}

Two hundred and two beam-column joint specimens were tested to study the anchorage behavior of headed bars. Key parameters included concrete compressive strength (3960 to 16,030 psi [27.3 to $110.6 \mathrm{MPa}$ ]), embedment length (4 to 19.25 in. [102 to $499 \mathrm{~mm}$ ]), head size (bearing areas from 3.8 to $14.9 A_{b}$ ), bar size (No. 5, No. 8, and No. 11 [No. 16, No. 25, and No. 36]), number of headed bars tested simultaneously within a specimen (two, three, or four), center-tocenter bar spacing between the headed bars (3 to $11.8 d_{b}$ ), and confining reinforcement within the joint region (ranging from none to six No. 3 [No. 10], bars spaced at $3 d_{b}$ ). Bar stresses at failure ranged from 26,100 to 153,200 psi (180 to $1057 \mathrm{MPa})$. Most of the headed bars met the requirements for Class HA heads in ASTM A970-13a, but some had large obstructions exceeding those requirements. The measured anchorage strengths were compared with the bar stresses calculated based on provisions for development length in the ACI Building Code (ACI 318-14).

The following conclusions are based on the data and analysis presented in this paper:

1. Specimens exhibited concrete breakout, side-face blowout failure modes, or a combination of the two, with most of the specimens exhibiting the concrete breakout failure.

2. A comparison of bar stress at failure with the stress calculated based on ACI 318-14 shows that ACI 318-14 provides a very conservative estimate of anchorage strength for No. 5 (No. 16) bars and low concrete compressive strengths and becomes progressively less conservative with increasing bar size and concrete compressive strength.

3. The provisions of ACI 318-14 should be adjusted to reflect the effects of concrete compressive strength, bar size, bar spacing, and confining reinforcement within the joint region over the broader range of values currently used in construction.

\section{AUTHOR BIOS}

ACI member Krishna P. Ghimire is a Teaching Assistant Professor of Civil Engineering at Kansas State University, Manhattan, KS. He received his BS from Tribhuvan University, Kirtipur, Nepal, and his MS and PhD from the University of Kansas, Lawrence, KS. He is a member of Joint ACI-ASCE Committee 408, Bond and Development of Steel Reinforcement.

Yun Shao is a Staff Professional with Kleinfelder, San Diego, CA. She received her BS and MS from Southeast University, Nanjing, China, and her PhD from the University of Kansas.

ACI Honorary Member David Darwin is the Deane E. Ackers Distinguished Professor and Chair of the Department of Civil, Environmental, and Architectural Engineering at the University of Kansas and a Past President of ACI. He is a member of ACI Committees 222, Corrosion of Metals in Concrete; 224, Cracking; Subcommittee 318-B, Anchorage and Reinforcement (Structural Concrete Building Code); and Joint ACI-ASCE Committees 408, Bond and Development of Steel Reinforcement; 445, Shear and Torsion; and 446, Fracture Mechanics of Concrete.

ACI member Matthew O'Reilly is a Chair's Council Assistant Professor of Civil, Environmental, and Architectural Engineering at the University of Kansas. He received his BS in mechanical engineering from the University of Rochester, Rochester, $N Y$, and his $M S$ and $P h D$ in civil engineering from the University of Kansas. He is a member of ACI Committees 123, Research and Current Developments; 222, Corrosion of Metals in Concrete; and Joint ACI-ASCE Committee 408, Bond and Development of Steel Reinforcement.

\section{ACKNOWLEDGMENTS}

Support for the study was provided by the Electric Power Research Institute, Concrete Reinforcing Steel Institute Education and Research Foundation, BarSplice Products, Inc., Headed Reinforcement Corp., and LENTON $^{\circledR}$ products from Pentair ${ }^{\circledR}$. Additional materials were supplied by Commercial Metals Company, Gerdau Corporation, Nucor Corporation, MMFX Technologies Corporation, Dayton Superior, Midwest Concrete Materials, and Grace Construction Products. Thanks are due to K. Barry, M. Ruis, and D. Scott, who provided project oversight for the Advanced Nuclear Technology Program of the Electric Power Research Institute; and to N. Anderson, C. Kopczynski, M. Mota, J. Munshi, and C. Paulson, who served as industry advisors. 


\section{REFERENCES}

ACI Committee 318, 2014, "Building Code Requirements for Structural Concrete (ACI 318-14) and Commentary (ACI 318R-14)," American Concrete Institute, Farmington Hills, MI, 520 pp.

ACI Committee 408, 2003, "Bond and Development of Straight Reinforcement in Tension (ACI 408R-03)," American Concrete Institute, Farmington Hills, MI, $49 \mathrm{pp}$

ASTM A1035/A1035M-15, 2015, "Standard Specification for Deformed and Plain, Low-Carbon, Chromium, Steel Bars for Concrete Reinforcement," ASTM International, West Conshohocken, PA, 7 pp.

ASTM A615/A615M-14, 2014, "Standard Specification for Deformed and Plain Carbon-Steel Bars for Concrete Reinforcement," ASTM International, West Conshohocken, PA, $7 \mathrm{pp}$.

ASTM A706/A706M-15, 2015, "Standard Specification for Deformed and Plain Low-Alloy Steel Bars for Concrete Reinforcement," ASTM International, West Conshohocken, PA, 7 pp.

ASTM A970/A970M-13a, 2013, "Standard Specification for Headed Steel Bars for Concrete Reinforcement," ASTM International, West Conshohocken, PA, 9 pp.
ASTM A970/A970M-18, 2018, "Standard Specification for Headed Steel Bars for Concrete Reinforcement," ASTM International, West Conshohocken, PA, $10 \mathrm{pp}$.

Draper, N. R., and Smith, H., 1981, Applied Regression Analysis, second edition, John Wiley and Sons, Wiley, New York, 241-249 pp.

Ghimire, K. P.; Darwin, D.; and O'Reilly, M., 2018, "Anchorage of Headed Reinforcing Bars in Concrete," SM Report No. 127, University of Kansas Center for Research, Lawrence, KS, Jan., 278 pp.

Marques, J. L., and Jirsa, J. O., 1975, "A Study of Hooked Bar Anchorages in Beam-Column Joints," ACI Journal Proceedings, V. 72, No. 5, May, pp. 198-209.

Shao, Y.; Darwin, D.; O’Reilly, M.; Lequesne, R. D.; Ghimire, K.; and Hano, M., 2016, "Anchorage of Conventional and High-Strength Headed Reinforcing Bars," SM Report No. 117, University of Kansas Center for Research, Lawrence, KS, Aug., 234 pp.

Sperry, J.; Yasso, S.; Searle, N.; DeRubeis, M.; Darwin, D.; O’Reilly, M.; Matamoros, A.; Feldman, L.; Lepage, A.; and Lequesne, R., 2017, "Conventional and High-Strength Hooked Bars-Part 1: Anchorage Tests," ACI Structural Journal, V. 114, No. 1, Jan.-Feb., pp. 255-266. doi: $10.14359 / 51689457$ 


\section{APPENDIX A: NOTATION, CONVERSION FACTORS, HEAD DETAILS, REINFORCEMENT LAYOUTS, AND DATA TABLES}

\section{A.1 NOTATION AND CONVERSION FACTORS}

\section{Notation}

$A_{a b} \quad$ Total cross-sectional area of all confining reinforcement parallel to $\ell_{d t}$ for headed bars being developed in beam-column joints and located within $8 d_{b}$ of the bottom (top) of the headed bars in direction of the outside of the joint for No. 3 through No. 8 headed bars or within $10 d_{b}$ of the bottom (top) of the bar in direction of the outside of the joint for No. 9 through No. 11 headed bars

$A_{b} \quad$ Area of an individual headed bar

$A_{b r g} \quad$ Net bearing area of the head of headed deformed bar

$A_{h s} \quad$ Total cross-sectional area of headed bars being developed

$A_{t r, l} \quad$ Area of single leg of confining reinforcement within joint region

$A_{t t} \quad$ Total cross-sectional area of all confining reinforcement parallel to $\ell_{d t}$ for headed bars being developed in beam-column joints and located within $8 d_{b}$ of the top (bottom) of the headed bars in direction of the interior of the joint for No. 3 through No. 8 headed bars or within $10 d_{b}$ of the top (bottom) of the bar in direction of the interior of the joint for No. 9 through No. 11 headed bars; or minimum total cross-sectional area of all confining reinforcement parallel to headed bars being developed in members other than beam-column joints within $71 / 2 d_{b}$ on one side of the bar centerline for No. 3 through No. 8 headed bars or within $91 / 2 d_{b}$ on one side of the bar centerline for No. 9 through No. 11 headed bars

$b \quad$ Width of column

$c_{b c} \quad$ Clear cover measured from the back of the head to the back of the member

$c_{h} \quad$ Clear spacing between adjacent headed bars

$c_{o} \quad$ Clear cover measured from the head to the side of the column

$c_{s b} \quad$ Clear cover measured from the bottom of the beam to the headed bar

$c_{s o} \quad$ Clear cover measured from the side of the headed bar to the side of the member

$c_{s o, a v g}$ Average clear side cover of the headed bars

$d_{b} \quad$ Nominal diameter of bar

$d_{\text {eff }} \quad$ Effective value of $d$ for beam-column joint

$d_{t r} \quad$ Nominal bar diameter of confining reinforcement within joint region

$d_{\text {tro }} \quad$ Nominal bar diameter of confining reinforcement outside joint region

$f_{c m} \quad$ Measured concrete compressive strength

$f_{s u} \quad$ Average stress in headed bars at failure

$f_{\text {su,max }}$ Maximum stress in individual headed bar

$h \quad$ Depth of column

$h_{c l} \quad$ Height measured from the center of the headed bar to the top of the bearing member

$\ell_{d t} \quad$ Development length in tension of headed deformed bar, measured from the critical section to the bearing face of the head

$\ell_{\text {eh }} \quad$ Embedment length measured from the bearing face of the head to the face of the member (or the end of the extended nodal zone, for CCT node specimens)

$\ell_{\text {eh,avg }}$ Average embedment length of headed bars

$n \quad$ Number of headed bars loaded simultaneously

$N \quad$ Number of legs of confining reinforcement in joint region

$T \quad$ Test failure load on a headed bar; average load on headed bars at failure

$T_{\text {ind }} \quad$ Peak load on individual headed bar at failure 
$T_{\max } \quad$ Maximum load on individual headed bar

$T_{\text {total }} \quad$ Sum of loads on headed bars at failure

$c_{c h} \quad$ Center-to-center spacing between adjacent headed bars

$S_{\text {tr }} \quad$ Center-to-center spacing of confining reinforcement (hoops) within joint region

Stro Center-to-center spacing of hoops outside joint region

\author{
Failure types \\ CB Concrete breakout \\ SB Side blowout \\ FP Local front pullout (secondary failure) \\ BS Back cover spalling (secondary failure) \\ Y Yield of headed bars
}

Specimen identification

(A@B) C-D-E-F\#G-H-I-J-K

A Number of headed bars in the specimen

B Center-to-center spacing between headed bars in terms of bar diameter

(A@B = blank, indicates specimens with 2 headed bars)

C ASTM in.-lb bar size

D Nominal compressive strength of concrete

E Head type

F Number of bars used as confining reinforcement within the joint region

G ASTM in.-lb bar size of confining reinforcement (if $\mathrm{F} \# \mathrm{G}=0$ = no confining reinforcement)

$\mathrm{H} \quad$ Hooked bars placed inside (i) or outside (o) of longitudinal reinforcement

I Nominal value of $c_{s o}$

$\mathrm{J} \quad$ Nominal value of $c_{b c}$

K Nominal value of $\ell_{\text {eh }}$

\title{
Conversion Factors
}

1 in. $=25.4 \mathrm{~mm}$

1 kip $=4.448 \mathrm{kN}$

$1 \mathrm{ksi}=6.895 \mathrm{MPa}$

$1 \mathrm{psi}=0.006895 \mathrm{MPa}$

Bar sizes: No. 3 (No. 10), No. 4 (No. 13), No. 5 (No. 16), No. 6 (No. 19), No. 7 (No. 22), No. 8

(No. 25), No. 9 (29), No. 10 (No. 32), No. 11 (No. 36) 


\section{A.2 DETAILS OF HEADS}

Table A.1 Details of friction-forged headed bars

\begin{tabular}{|c|c|c|c|c|c|c|c|c|c|}
\hline & Dimension Notation & Designation & $\begin{array}{l}\text { Bar } \\
\text { Size }\end{array}$ & $\begin{array}{c}b \\
\text { (in.) }\end{array}$ & $\begin{array}{c}h \\
\text { (in.) }\end{array}$ & $\begin{array}{c}t \\
\text { (in.) }\end{array}$ & $\begin{array}{l}d_{o b s} \\
\text { (in.) }{ }^{[1]}\end{array}$ & $\begin{array}{c}t_{o b s} \\
\text { (in.) }{ }^{[1]}\end{array}$ & $\begin{array}{c}\text { Net } \\
\text { Bearing } \\
\text { Area } \\
\left(A_{b r g}\right)^{[2]}\end{array}$ \\
\hline \multirow{6}{*}{ 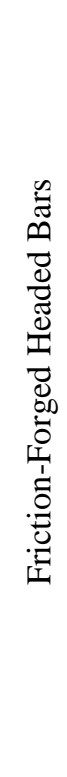 } & & F4.0 & No. 5 & 1.25 & 1.25 & 0.5 & 0.93 & 0.46 & $4.1 A_{b}$ \\
\hline & & F4.1 $1^{[3]}$ & No. 8 & 2.02 & 2 & 1 & 1.54 & 0.60 & $4.1 A_{b}$ \\
\hline & & F3.8 & No. 11 & 2.5 & 3 & 1.375 & 2.11 & 0.73 & $3.8 A_{b}$ \\
\hline & & F13.1 & No. 5 & 1.25 & 3.5 & 0.5 & 0.93 & 0.46 & $13.1 A_{b}$ \\
\hline & & F9.1 $1^{[3]}$ & No. 8 & 2.03 & 3.98 & 1.02 & 1.58 & 0.62 & $9.2 A_{b}$ \\
\hline & & F8.6 & No. 11 & 2.5 & 6 & 1.375 & 2.11 & 0.73 & $8.6 A_{b}$ \\
\hline
\end{tabular}

[1] See Fig. A1

[2] Net bearing area calculated as gross head area minus bar area. These heads contained obstructions adjacent to the bearing face of the head, as shown in Fig. A1, A3b, and A3c. These obstructions, however, did not have any detrimental effects on the anchorage strength of the headed bars and, therefore, are not considered to detract from the net bearing area of the head (Ghimire et al. 2018).

[3] Head dimensions updated from those given by Shao et al. (2016) (Ghimire et al. 2018)

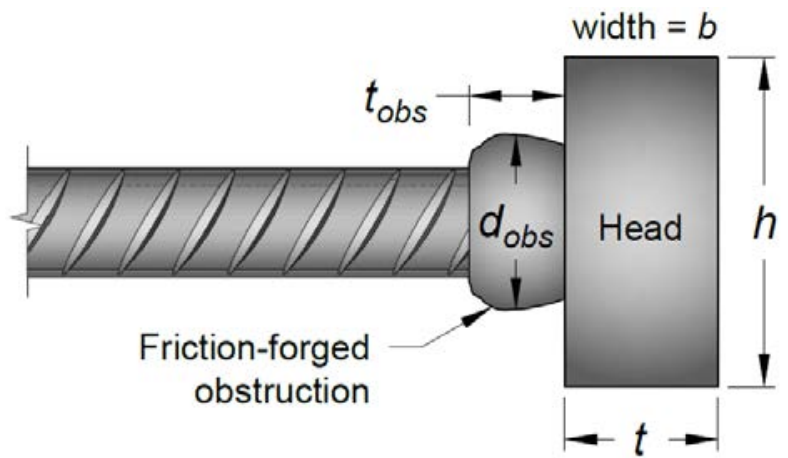

Fig. A1 Friction-forged obstruction adjacent to the bearing face of the head 
Table A.1 Cont. Details of taper-threaded and cold-swaged headed bars

\begin{tabular}{|c|c|c|c|c|c|c|}
\hline & Dimension Notation & Designation & $\begin{array}{l}\text { Bar } \\
\text { Size }\end{array}$ & $d$ (in.) & $t$ (in.) & $\begin{array}{l}\text { Net Bearing } \\
\text { Area }\left(A_{b r g}\right)^{[1]}\end{array}$ \\
\hline \multirow{2}{*}{ 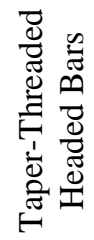 } & & T4.0 & No. 8 & 2.25 & 1.5 & $4.0 A_{b}$ \\
\hline & & T9.5 & No. 8 & 3.25 & 1.5 & $9.5 A_{b}$ \\
\hline \multirow{5}{*}{ 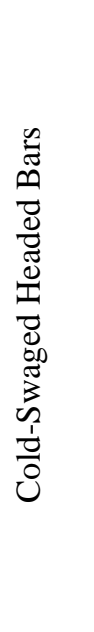 } & & $\mathrm{S} 4.0^{[2][3]}$ & No. 6 & 1.63 & 1.52 & $4.1 A_{b}$ \\
\hline & & S6. $5^{[2][3]}$ & No. 8 & 2.4 & 1.78 & $5.0 A_{b}$ \\
\hline & & $\mathrm{S} 5.5^{[3]}$ & No. 11 & 3.5 & 2.75 & $5.5 A_{b}$ \\
\hline & \multirow{2}{*}{$d$} & S9.5 & No. 8 & 3.25 & 2.75 & $9.5 A_{b}$ \\
\hline & & $\mathrm{S} 14.9^{[2]}$ & No. 8 & 4.01 & 2.66 & $15 A_{b}$ \\
\hline
\end{tabular}

[1] Net bearing area calculated as gross head area minus bar area

[2] Head dimensions updated from those given by Shao et al. (2016) (Ghimire et al. 2018)

[3] Octagonal head 
Table A.1 Cont. Details of cold-swaged threaded coupling sleeve headed bars

\begin{tabular}{|c|c|c|c|c|c|c|c|c|}
\hline & Dimension Notation & Designation & $\begin{array}{l}\text { Bar } \\
\text { Size }\end{array}$ & $\begin{array}{c}d_{1} \\
\text { (in.) }\end{array}$ & $\begin{array}{c}t_{1} \\
\text { (in.) }\end{array}$ & $\begin{array}{c}d_{2} \\
\text { (in.) }\end{array}$ & $\begin{array}{c}t_{2} \\
\text { (in.) }\end{array}$ & $\begin{array}{c}\text { Net } \\
\text { Bearing } \\
\text { Area }\left(A_{b r g}\right)\end{array}$ \\
\hline \multirow{8}{*}{ 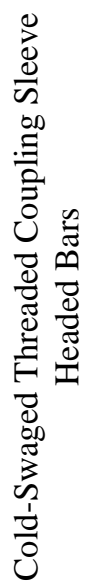 } & \multirow{8}{*}{$d_{1}(1) \frac{d_{2}}{x}$} & \multirow{2}{*}{$\mathrm{O} 4.5^{[1]}$} & \multirow{2}{*}{ No. 8} & \multirow{2}{*}{2.76} & \multirow{2}{*}{1.625} & $1.72^{[2]}$ & \multirow{2}{*}{5.19} & $4.6 A_{b}{ }^{[4]}$ \\
\hline & & & & & & $2.2^{[3]}$ & & $2.8 A_{b}{ }^{[5]}$ \\
\hline & & \multirow{2}{*}{$\mathrm{O} 4.5^{[1]}$} & \multirow{2}{*}{ No. 11} & \multirow{2}{*}{3.75} & \multirow{2}{*}{2.17} & $2.28^{[2]}$ & \multirow{2}{*}{6.69} & $4.5 A_{b}{ }^{[4]}$ \\
\hline & & & & & & $2.86^{[3]}$ & & $3.0 A_{b}{ }^{[5]}$ \\
\hline & & \multirow{2}{*}{ O9.1 $1^{[1]}$} & \multirow{2}{*}{ No. 8} & \multirow{2}{*}{3.5} & \multirow{2}{*}{1.625} & $1.72^{[2]}$ & \multirow{2}{*}{5.19} & $9.2 A_{b}{ }^{[4]}$ \\
\hline & & & & & & $2.2^{[3]}$ & & $7.4 A_{b}{ }^{5]}$ \\
\hline & & \multirow{2}{*}{ O12.9 $9^{[1]}$} & \multirow{2}{*}{ No. 8} & \multirow{2}{*}{4} & \multirow{2}{*}{1.625} & $1.72^{[2]}$ & \multirow{2}{*}{5.19} & $13.0 A_{b}{ }^{[4]}$ \\
\hline & & & & & & $2.2^{[3]}$ & & $11.2 A_{b}[5]$ \\
\hline
\end{tabular}

[1] Head dimensions updated from those given by Shao et al. (2016). These heads contained obstructions with a gap (width not less than $3 / 8$ in.) adjacent to the bearing face of the head, as shown in Fig. A2 (Ghimire et al. 2018).

[2] Based on size of obstruction adjacent to head

[3] Based on maximum size of obstruction

[4] Net bearing area calculated as gross head area minus area of the obstruction adjacent to the bearing face of the head

[5] Net bearing area calculated as gross head area minus the maximum area of the obstruction

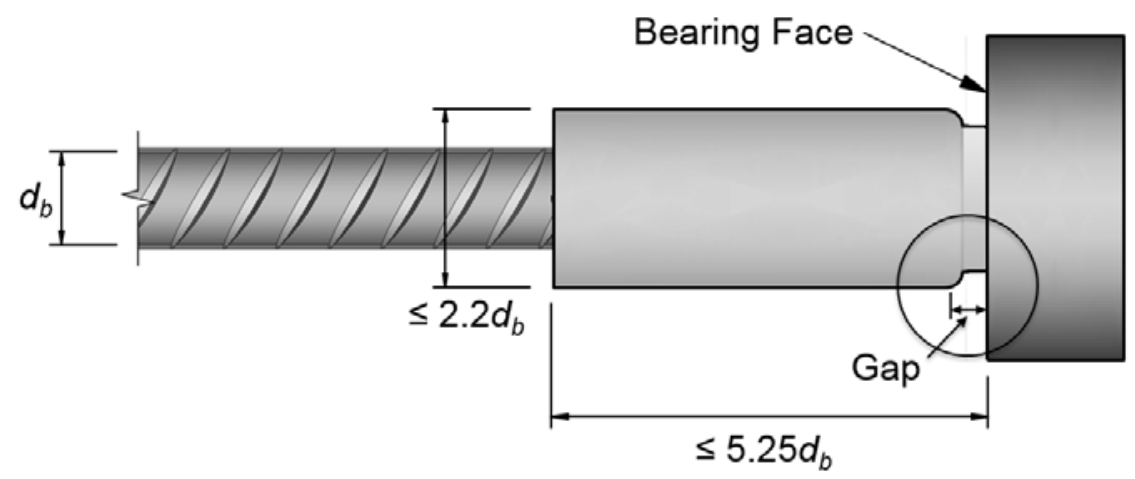

Fig. A2 Maximum dimensions and non-planner features of the obstruction 


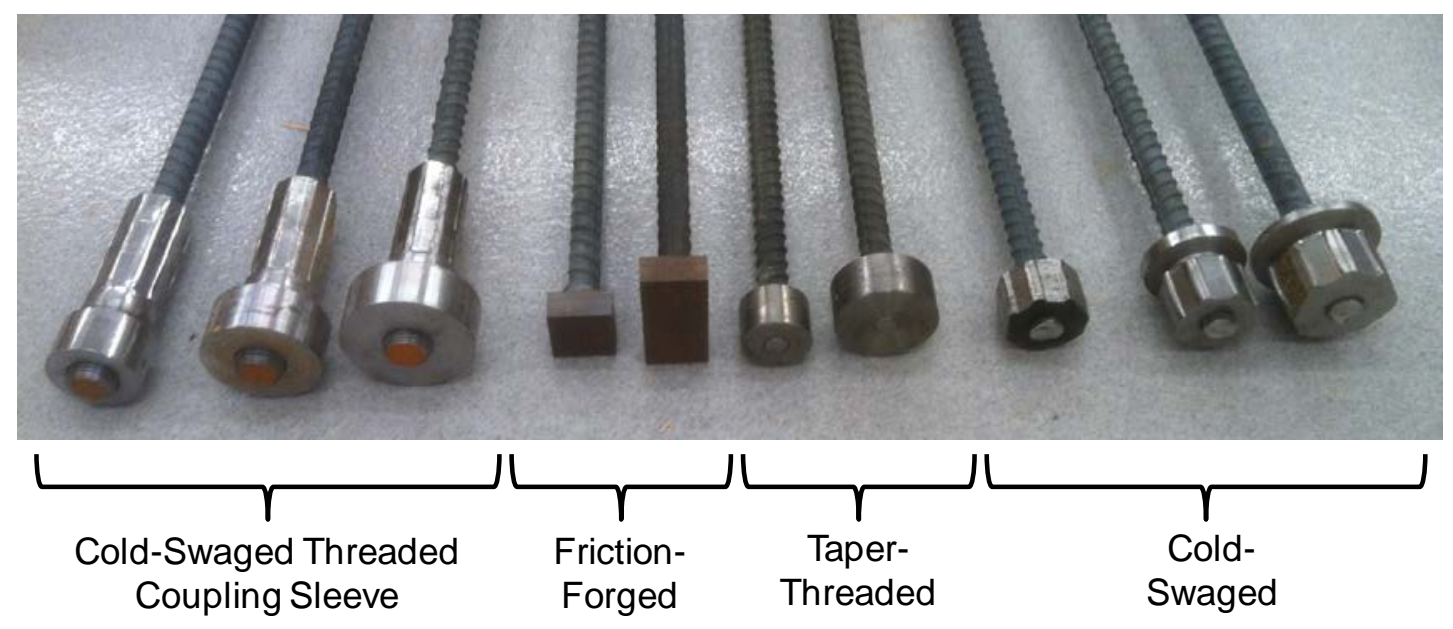

(a)

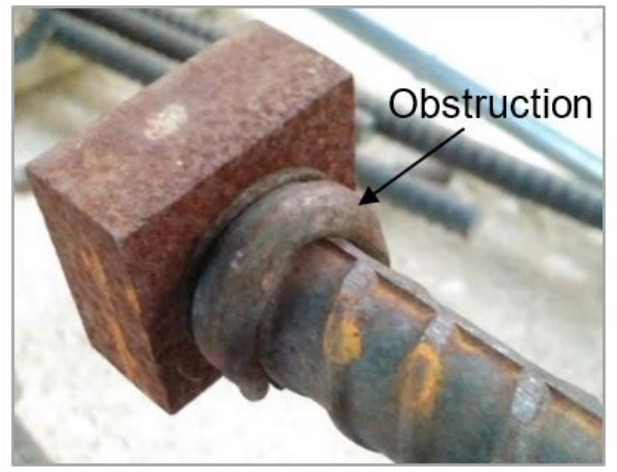

(b)

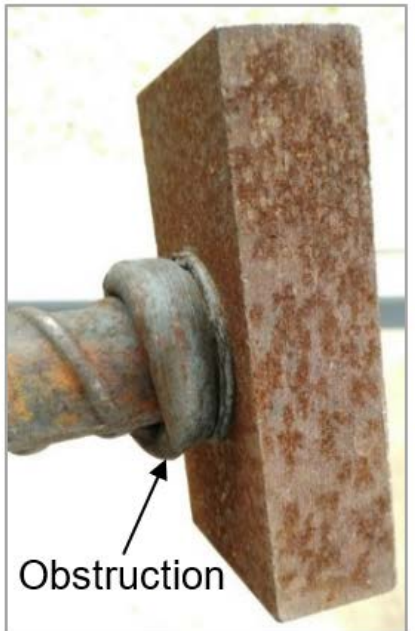

(c)

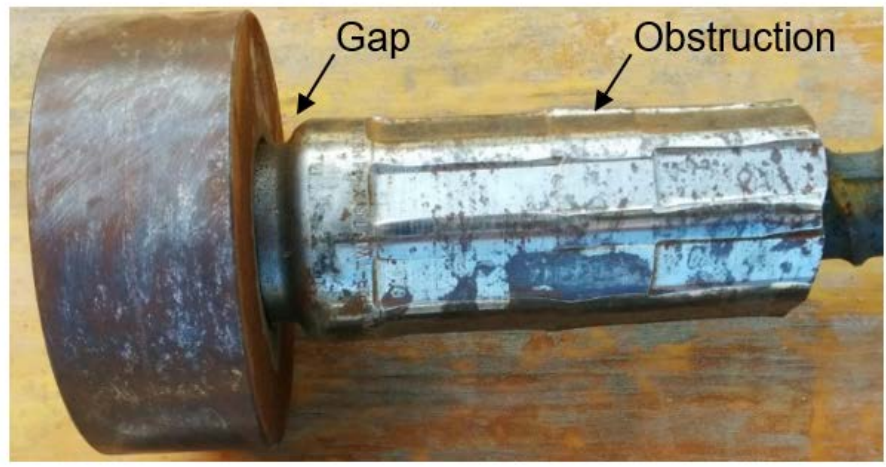

(d)

Fig. A3 (a) Left to right: cold-swaged threaded coupling sleeve, friction-forged, taper-threaded, and cold-swaged headed bars; (b) and (c) obstruction adjacent to the bearing face of the frictionforged heads; and (d) gap between the obstruction adjacent to the bearing face of the cold-swaged threaded coupling sleeve heads (taper-threaded and cold-swaged heads had no obstructions) 


\section{A.3 SPECIMENS CONTAINING CROSSTIES AND HAIRPINS WITHIN JOINT REGION}

No. 3 longitudinal bars

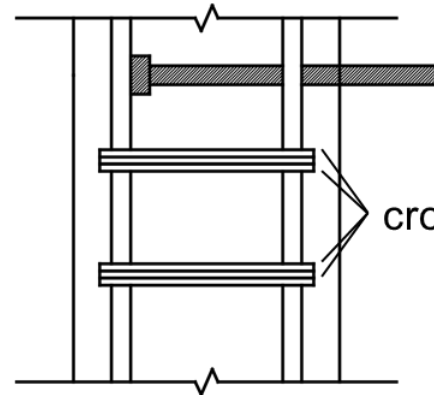

(a)

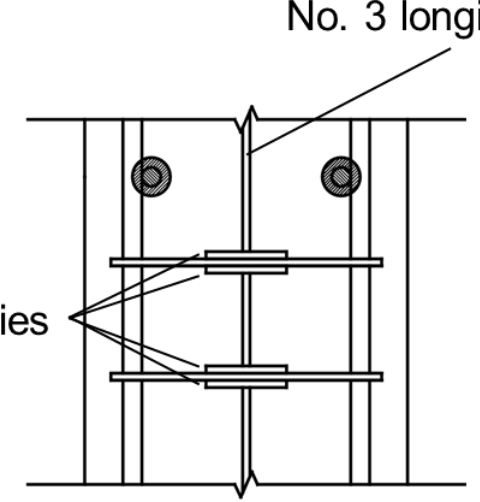

(b)

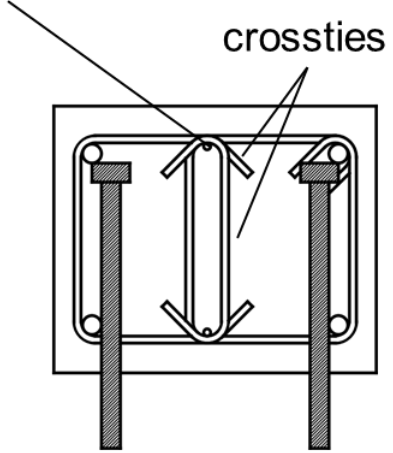

(c)

Fig. A4 Specimen containing crossties within joint region

(a) side view, (b) front view, (c) top view

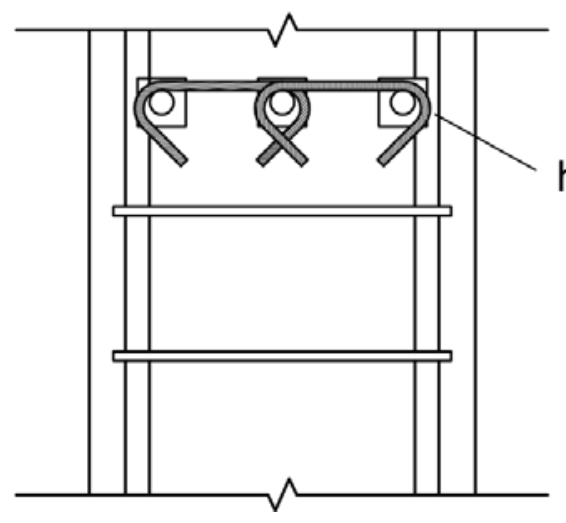

(a)

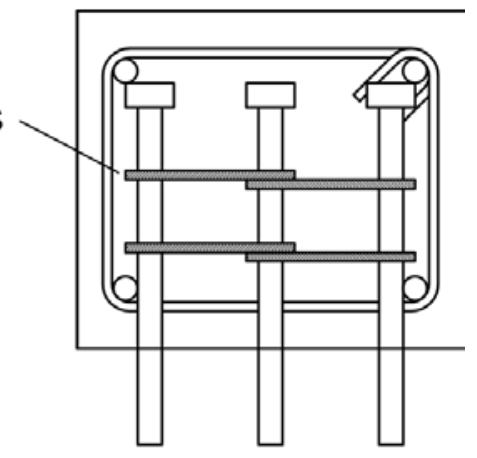

(b)

Fig. A5 Specimen with hairpin reinforcement (a) front view, (b) top view 


\section{A.4 LONGITUDINAL COLUMN STEEL LAYOUTS}

The longitudinal column reinforcement layouts A1 through A17 shown below may not reflect the real size, number, and location of headed bars. Confining reinforcement is omitted in the layouts for clarity.

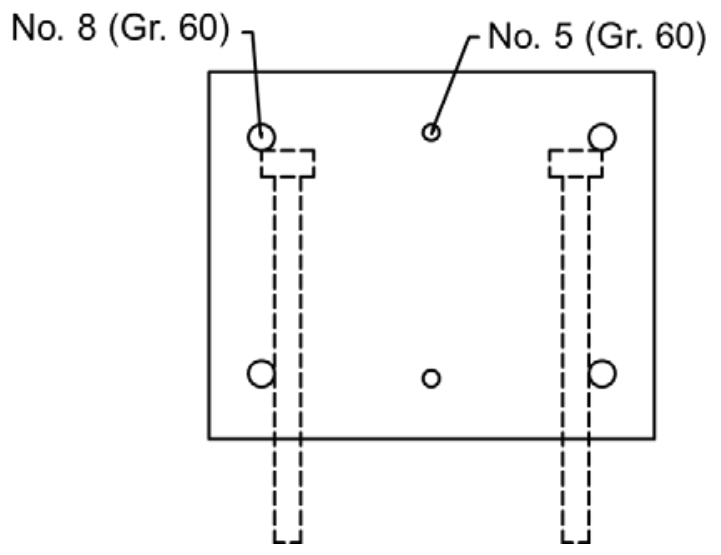

Layout A1: 4 No. $8+2$ No. 5 (Gr. 60)

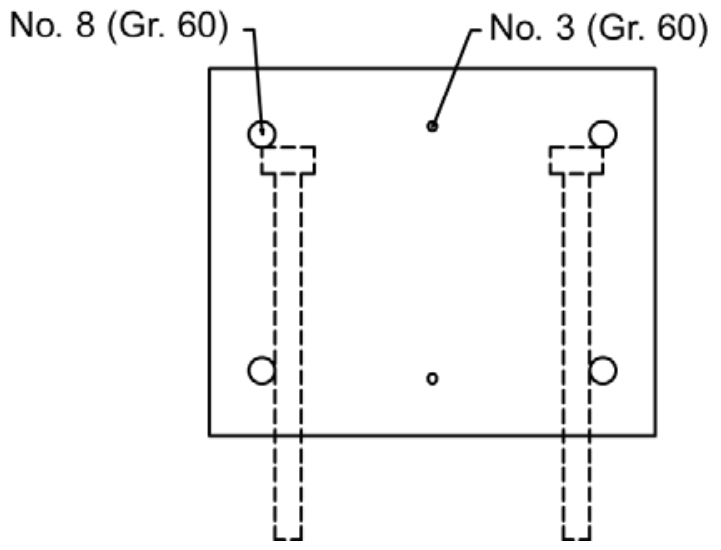

Layout A2: 4 No. 8 + 2 No. 3 (Gr. 60) 
No. 8 (Gr. 60)

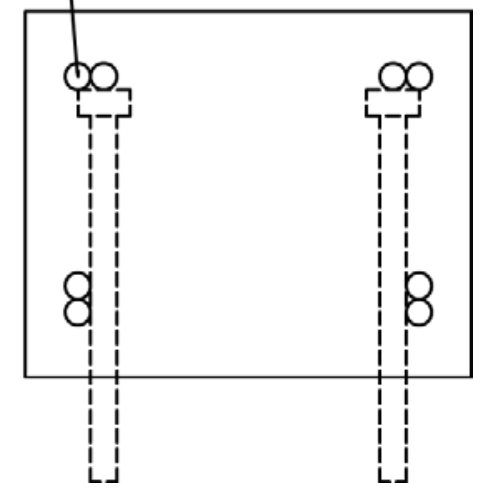

Layout A3: 8 No. 8 (Gr. 60), bundled at corner

No. 8 (Gr. 60)

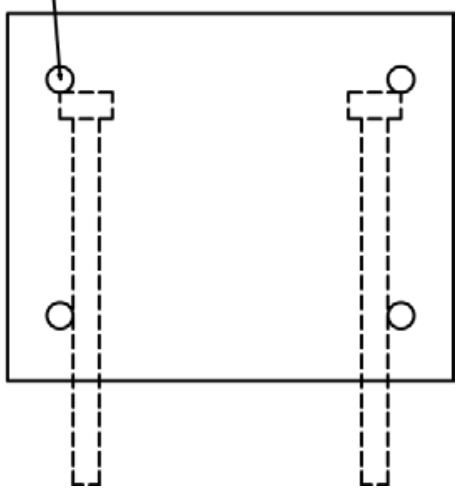

Layout A4: 4 No. 8 (Gr. 60)

No. 8 (Gr. 120)

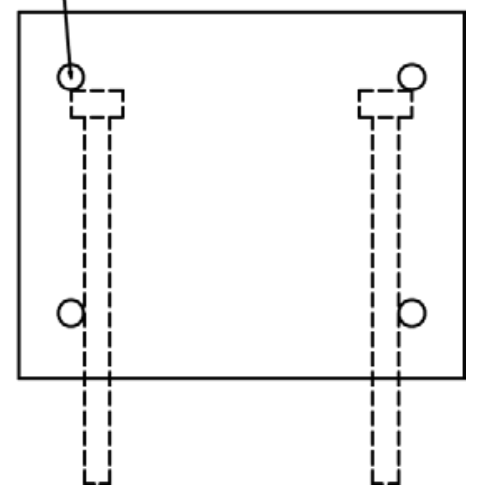

Layout A5: 4 No. 8 (Gr. 120) 
No. 8 (Gr. 60)

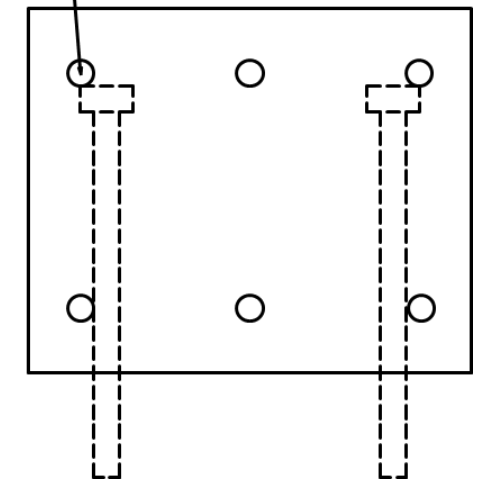

Layout A6: 6 No. 8 (Gr. 60)

No. 8 (Gr. 60)

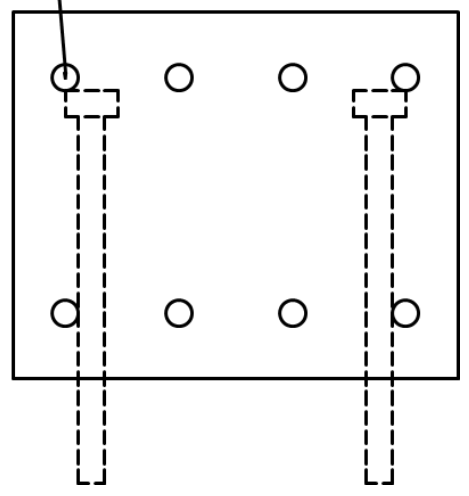

Layout A7: 8 No. 8 (Gr. 60)

No. 8 (Gr. 60)

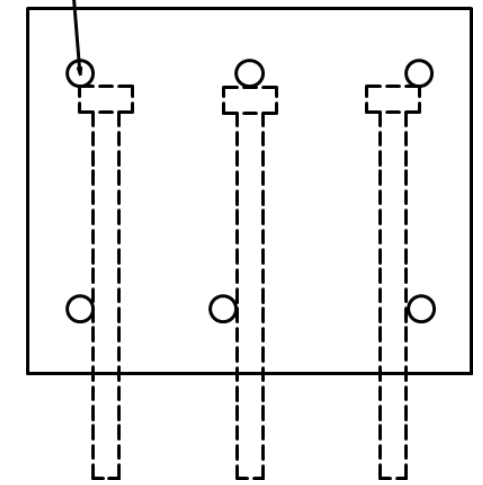

Layout A8: 6 No. 8 (Gr. 60), non-symmetric 


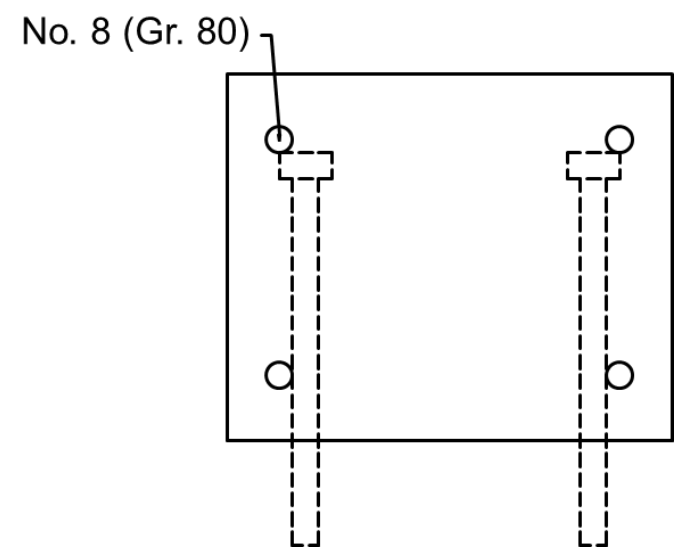

Layout A9: 4 No. 8 (Gr. 80)

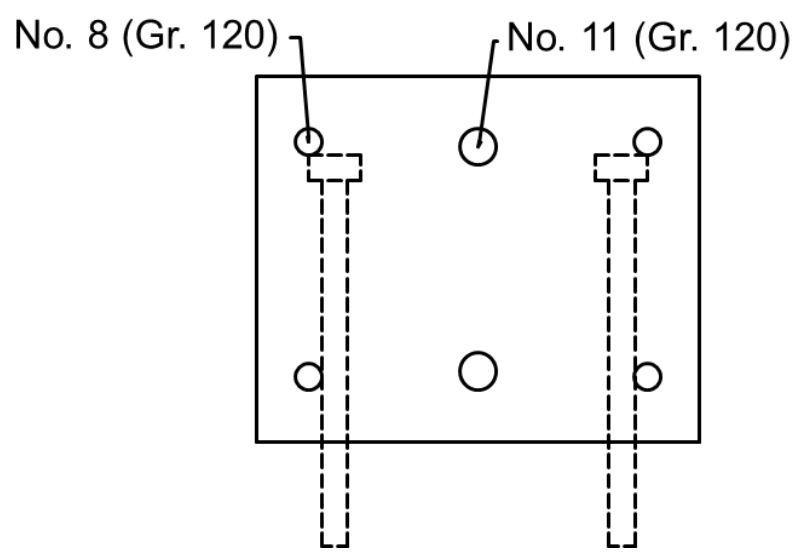

Layout A10: 4 No. 8 + 2 No. 11 (Gr. 120)

No. 8 (Gr. 120)

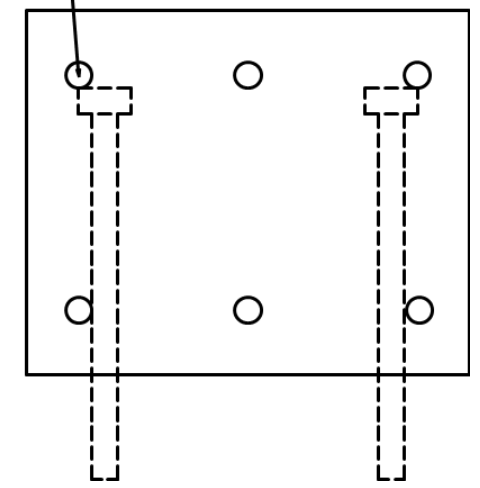

Layout A11: 6 No. 8 (Gr. 120) 
No. 8 (Gr. 60)

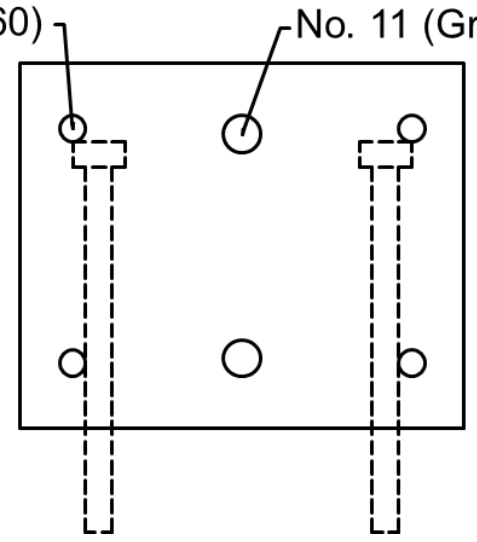

Layout A12: 4 No. 8 (Gr. 60) + 2 No. 11 (Gr. 80)

No. 8 (Gr. 120)

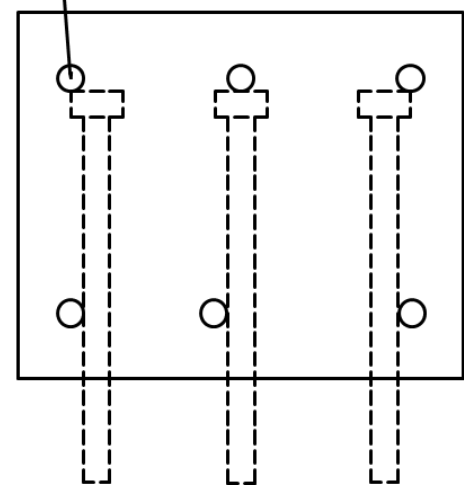

Layout A13: 6 No. 8 (Gr. 120), non-symmetric

No. 8 (Gr. 120)

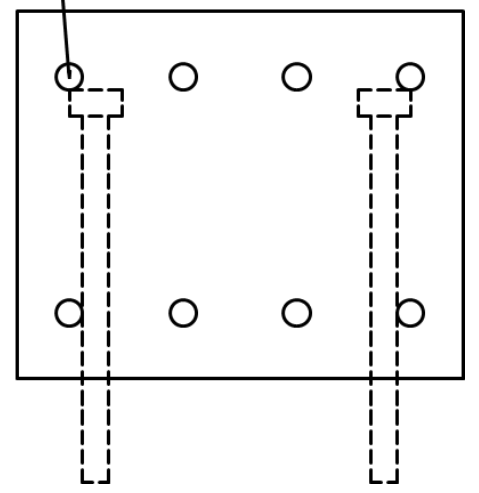

Layout A14: 8 No. 8 (Gr. 120) 


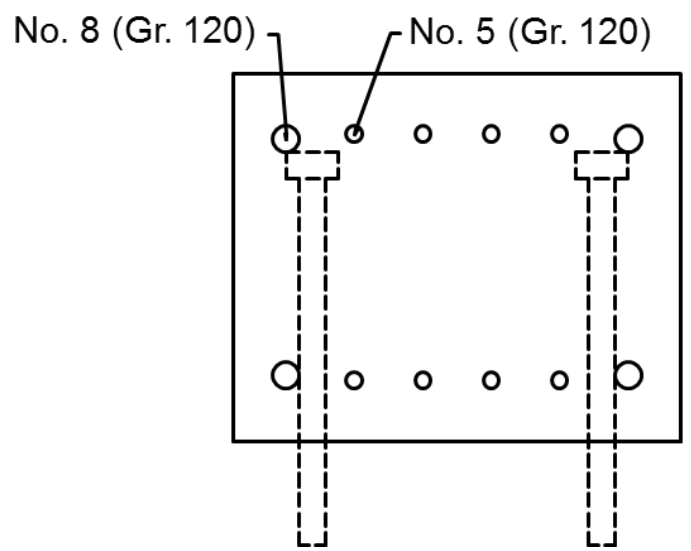

Layout A15: 4 No. 8 +8 No. 5 (Gr. 120)

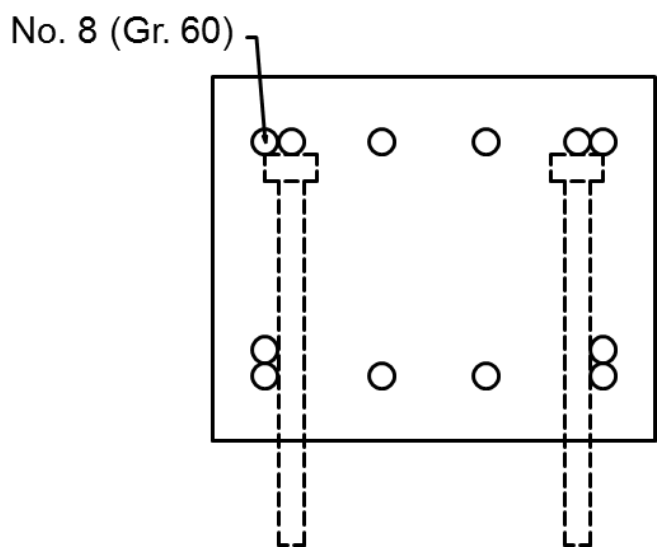

Layout A16: 12 No. 8 (Gr. 60)

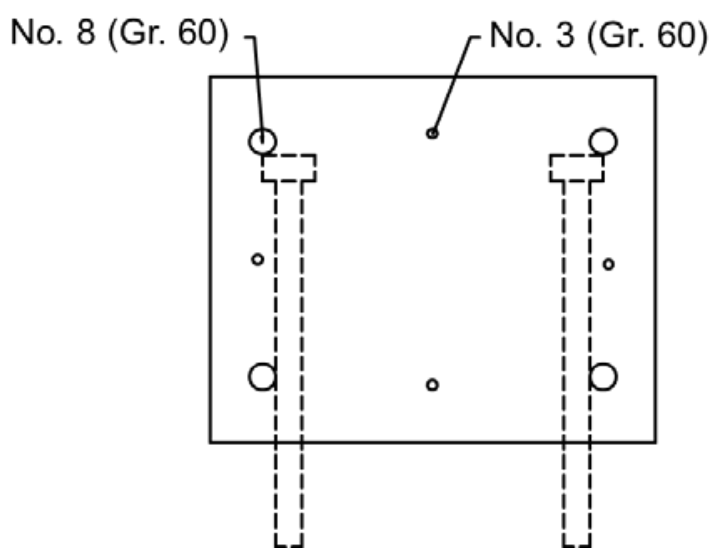

Layout A17: 4 No. 8 + 4 No. 3 (Gr. 60) 


\section{A.5 COMPREHENSIVE TEST RESULTS}

Table A.2 Comprehensive test results and data for beam-column joint specimens

\begin{tabular}{|c|c|c|c|c|c|c|c|c|c|c|}
\hline & Specimen & Head & $\begin{array}{l}\boldsymbol{c}_{\boldsymbol{o}} \\
\text { in. }\end{array}$ & $A_{b r g}$ & $\begin{array}{l}\ell \text { eh } \\
\text { in. }\end{array}$ & $\begin{array}{c}\ell \text { eh, avg } \\
\text { in. }\end{array}$ & $\begin{array}{l}\boldsymbol{f}_{\boldsymbol{c m}} \\
\mathrm{psi}\end{array}$ & $\begin{array}{l}\text { Age } \\
\text { days }\end{array}$ & $\begin{array}{l}\boldsymbol{d}_{\boldsymbol{b}} \\
\text { in. }\end{array}$ & $\begin{array}{c}\boldsymbol{A}_{\boldsymbol{b}} \\
\text { in. }^{2}\end{array}$ \\
\hline \multirow{6}{*}{ Group 1} & $8-5-T 4.0-0-i-3-3-15.5^{a b}$ & $\begin{array}{l}\text { A } \\
\text { B }\end{array}$ & 2.4 & $4.0 A_{b}$ & $\begin{array}{l}15.50 \\
16.00 \\
\end{array}$ & 15.75 & 4850 & 7 & 1 & 0.79 \\
\hline & $8-5-T 4.0-0-i-4-3-15.5^{\mathrm{a}}$ & $\begin{array}{l}\text { A } \\
\text { B }\end{array}$ & 3.4 & $4.0 A_{b}$ & $\begin{array}{l}15.50 \\
15.06 \\
\end{array}$ & 15.28 & 5070 & 8 & 1 & 0.79 \\
\hline & $8-5-T 4.0-4 \# 3-i-3-3-12.5^{a}$ & $\begin{array}{l}\text { A } \\
B\end{array}$ & 2.4 & $4.0 A_{b}$ & $\begin{array}{l}12.06 \\
12.69 \\
\end{array}$ & 12.38 & 5070 & 8 & 1 & 0.79 \\
\hline & $8-5-T 4.0-4 \# 3-i-4-3-12.5^{a}$ & $\begin{array}{l}A \\
B\end{array}$ & 3.4 & $4.0 A_{b}$ & $\begin{array}{l}11.94 \\
12.19\end{array}$ & 12.06 & 5380 & 11 & 1 & 0.79 \\
\hline & $8-5-T 4.0-4 \# 4-i-3-3-12.5^{a}$ & $\begin{array}{l}A \\
B\end{array}$ & 2.4 & $4.0 A_{b}$ & $\begin{array}{l}12.56 \\
12.31\end{array}$ & 12.44 & 5070 & 8 & 1 & 0.79 \\
\hline & $8-5-T 4.0-4 \# 4-i-4-3-12.5^{a}$ & $\begin{array}{l}A \\
B\end{array}$ & 3.4 & $4.0 A_{b}$ & $\begin{array}{l}12.06 \\
12.31\end{array}$ & 12.19 & 4850 & 7 & 1 & 0.79 \\
\hline \multirow{6}{*}{ Group 2} & $8-5 g-T 4.0-0-i-2.5-3-12.5^{a}$ & $\begin{array}{l}\text { A } \\
\text { B }\end{array}$ & 1.9 & $4.0 A_{b}$ & $\begin{array}{l}12.69 \\
12.44 \\
\end{array}$ & 12.56 & 5910 & 14 & 1 & 0.79 \\
\hline & $8-5 g-T 4.0-0-i-3.5-3-12.5^{\mathrm{a}}$ & $\begin{array}{l}\text { A } \\
\text { B }\end{array}$ & 2.9 & $4.0 A_{b}$ & $\begin{array}{l}12.44 \\
12.56 \\
\end{array}$ & 12.50 & 6320 & 15 & 1 & 0.79 \\
\hline & 8-5g-T4.0-5\#3-i-2.5-3-9.5 & $\begin{array}{l}\text { A } \\
\text { B }\end{array}$ & 1.9 & $4.0 A_{b}$ & $\begin{array}{l}9.44 \\
9.69\end{array}$ & 9.56 & 5090 & 7 & 1 & 0.79 \\
\hline & 8-5g-T4.0-5\#3-i-3.5-3-9.5 & $\begin{array}{l}\text { A } \\
B\end{array}$ & 2.9 & $4.0 A_{b}$ & $\begin{array}{l}9.69 \\
9.44\end{array}$ & 9.56 & 5910 & 14 & 1 & 0.79 \\
\hline & $8-5 g-T 4.0-4 \# 4-i-2.5-3-9.5^{a}$ & $\begin{array}{l}\text { A } \\
B\end{array}$ & 1.9 & $4.0 A_{b}$ & $\begin{array}{l}9.44 \\
8.94 \\
\end{array}$ & 9.19 & 5180 & 8 & 1 & 0.79 \\
\hline & $8-5 g-T 4.0-4 \# 4-i-3.5-3-9.5^{\mathrm{a}}$ & $\begin{array}{l}\text { A } \\
\text { B }\end{array}$ & 2.9 & $4.0 A_{b}$ & $\begin{array}{l}9.31 \\
9.69 \\
\end{array}$ & 9.50 & 5910 & 14 & 1 & 0.79 \\
\hline \multirow{6}{*}{ Group 3} & $8-5-T 4.0-0-i-2.5-3-12.5^{a}$ & $\begin{array}{l}\text { A } \\
\text { B }\end{array}$ & 1.9 & $4.0 A_{b}$ & $\begin{array}{l}12.69 \\
12.50 \\
\end{array}$ & 12.59 & 6210 & 8 & 1 & 0.79 \\
\hline & $8-5-T 4.0-0-i-3.5-3-12.5^{a}$ & $\begin{array}{l}\text { A } \\
\text { B }\end{array}$ & 2.9 & $4.0 A_{b}$ & $\begin{array}{l}12.81 \\
12.50 \\
\end{array}$ & 12.66 & 6440 & 9 & 1 & 0.79 \\
\hline & $8-5-T 4.0-5 \# 3-i-2.5-3-9.5^{a}$ & $\begin{array}{l}\text { A } \\
\text { B }\end{array}$ & 1.9 & $4.0 A_{b}$ & $\begin{array}{l}9.44 \\
9.19 \\
\end{array}$ & 9.31 & 5960 & 7 & 1 & 0.79 \\
\hline & $8-5-T 4.0-5 \# 3-i-3.5-3-9.5^{a}$ & $\begin{array}{l}\text { A } \\
B\end{array}$ & 2.9 & $4.0 A_{b}$ & $\begin{array}{l}9.06 \\
9.06 \\
\end{array}$ & 9.06 & 6440 & 9 & 1 & 0.79 \\
\hline & $8-5-T 4.0-4 \# 4-i-2.5-3-9.5^{\mathrm{a}}$ & $\begin{array}{l}\text { A } \\
\text { B }\end{array}$ & 1.9 & $4.0 A_{b}$ & $\begin{array}{l}9.19 \\
9.31\end{array}$ & 9.25 & 6440 & 9 & 1 & 0.79 \\
\hline & $8-5-T 4.0-4 \# 4-i-3.5-3-9.5^{a}$ & $\begin{array}{l}\text { A } \\
\text { B }\end{array}$ & 2.9 & $4.0 A_{b}$ & $\begin{array}{l}9.56 \\
8.94\end{array}$ & 9.25 & 6210 & 8 & 1 & 0.79 \\
\hline \multirow{3}{*}{ Group 4} & 8-8-F4.1-0-i-2.5-3-10.5 & $\begin{array}{l}\text { A } \\
\text { B }\end{array}$ & 2.0 & $4.1 A_{b}$ & $\begin{array}{l}10.25 \\
10.75 \\
\end{array}$ & 10.50 & 8450 & 9 & 1 & 0.79 \\
\hline & 8-8-F4.1-2\#3-i-2.5-3-10 & $\begin{array}{l}\text { A } \\
\text { B }\end{array}$ & 2.0 & $4.1 A_{b}$ & $\begin{array}{c}9.75 \\
10.00 \\
\end{array}$ & 9.88 & 8450 & 9 & 1 & 0.79 \\
\hline & (3@3)8-8-F4.1-0-i-2.5-3-10.5 & $\begin{array}{l}\text { A } \\
B \\
C\end{array}$ & 2.0 & $4.1 A_{b}$ & $\begin{array}{l}10.63 \\
10.75 \\
10.38\end{array}$ & 10.58 & 8450 & 9 & 1 & 0.79 \\
\hline
\end{tabular}

a Specimen contained crossties within joint region

${ }^{\mathrm{b}}$ Specimen had no confining reinforcement above joint region 
Table A.2 Cont. Comprehensive test results and data for beam-column joint specimens

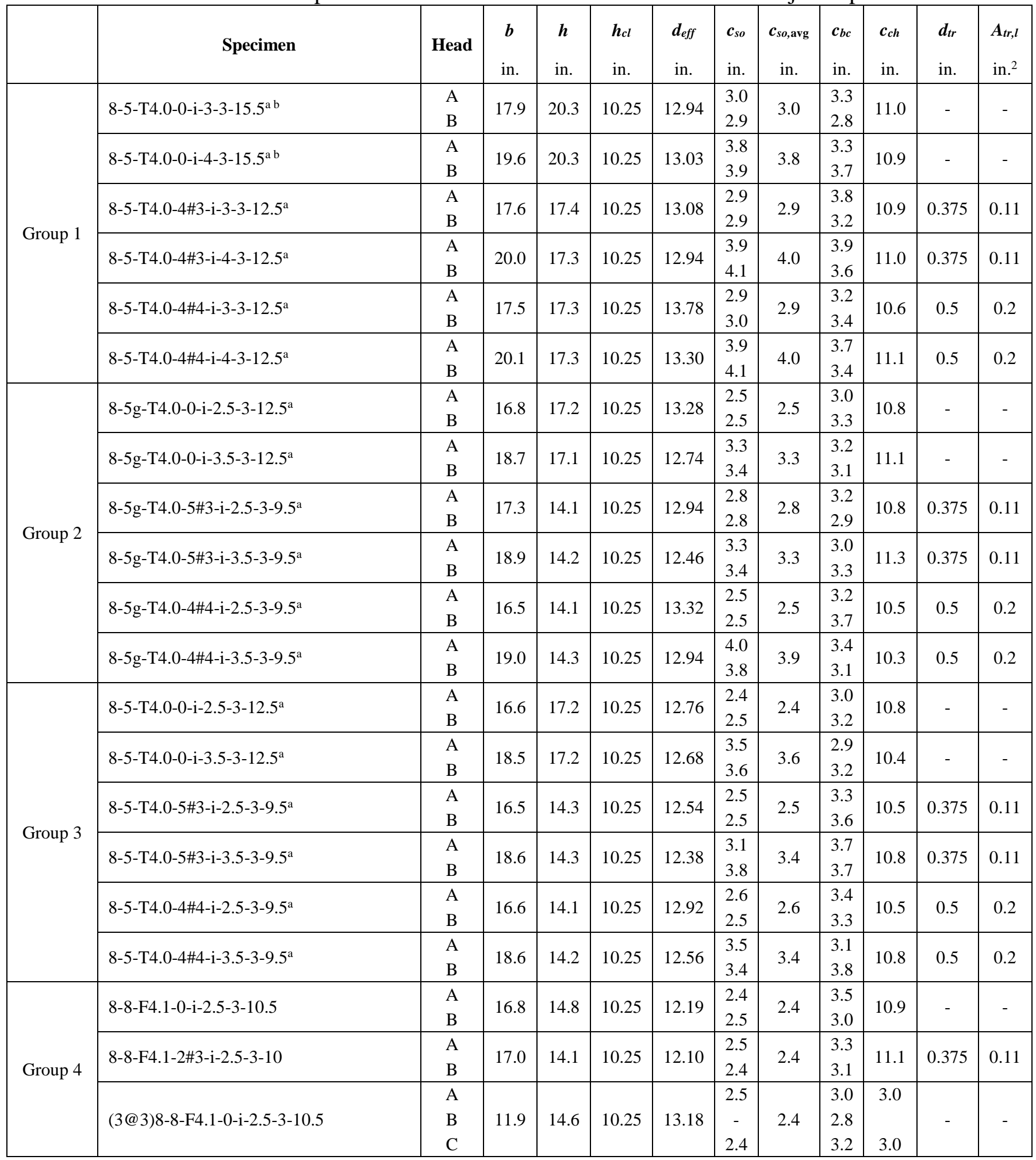

a Specimen contained crossties within joint region

b Specimen had no confining reinforcement above joint region 
Table A.2 Cont. Comprehensive test results and data for beam-column joint specimens

\begin{tabular}{|c|c|c|c|c|c|c|c|c|c|c|c|}
\hline & Specimen & Head & $N$ & $\begin{array}{l}s_{t r}{ }^{c} \\
\text { in. }\end{array}$ & $\begin{array}{l}\boldsymbol{A}_{t t} \\
\text { in. }^{2}\end{array}$ & $\begin{array}{l}\boldsymbol{d}_{\text {tro }} \\
\text { in. }\end{array}$ & $\begin{array}{l}S_{t r o}{ }^{c} \\
\text { in. }\end{array}$ & $\begin{array}{l}A_{a b} \\
\text { in. }^{2}\end{array}$ & $n$ & $\begin{array}{l}A_{h s} \\
\text { in. }^{2}\end{array}$ & $\begin{array}{l}\text { Long. } \\
\text { Reinf. } \\
\text { Layout }\end{array}$ \\
\hline \multirow{6}{*}{ Group 1} & $8-5-T 4.0-0-i-3-3-15.5^{a}$ & $\begin{array}{l}\mathrm{A} \\
\mathrm{B}\end{array}$ & - & - & - & - & - & - & 2 & 1.58 & A1 \\
\hline & $8-5-T 4.0-0-i-4-3-15.5^{a}$ & $\begin{array}{l}\text { A } \\
B\end{array}$ & - & - & - & - & - & - & 2 & 1.58 & A1 \\
\hline & 8-5-T4.0-4\#3-i-3-3-12.5 & $\begin{array}{l}\text { A } \\
B\end{array}$ & 8 & $\begin{array}{c}3 \\
(1.5) \\
\end{array}$ & 0.66 & 0.375 & $\begin{array}{c}3 \\
(1.5) \\
\end{array}$ & 0.66 & 2 & 1.58 & A2 \\
\hline & $8-5-T 4.0-4 \# 3-\mathrm{i}-4-3-12.5^{\mathrm{a}}$ & $\begin{array}{l}\text { A } \\
B\end{array}$ & 8 & $\begin{array}{c}3 \\
(1.5) \\
\end{array}$ & 0.66 & 0.375 & $\begin{array}{c}3 \\
(1.5) \\
\end{array}$ & 0.66 & 2 & 1.58 & A2 \\
\hline & $8-5-T 4.0-4 \# 4-i-3-3-12.5^{\mathrm{a}}$ & $\begin{array}{l}\mathrm{A} \\
\mathrm{B}\end{array}$ & 8 & $\begin{array}{c}4 \\
(2) \\
\end{array}$ & 0.80 & 0.5 & $\begin{array}{c}4 \\
(2) \\
\end{array}$ & 0.80 & 2 & 1.58 & A17 \\
\hline & 8-5-T4.0-4\#4-i-4-3-12.5 & $\begin{array}{l}\text { A } \\
B\end{array}$ & 8 & $\begin{array}{c}4 \\
(2) \\
\end{array}$ & 0.80 & 0.5 & $\begin{array}{c}4 \\
(2) \\
\end{array}$ & 0.80 & 2 & 1.58 & A17 \\
\hline \multirow{6}{*}{ Group 2} & $8-5 g-T 4.0-0-i-2.5-3-12.5^{a}$ & $\begin{array}{l}\mathrm{A} \\
\mathrm{B}\end{array}$ & - & - & - & 0.375 & $\begin{array}{c}3.5 \\
(1.75)\end{array}$ & 0.44 & 2 & 1.58 & A2 \\
\hline & 8-5g-T4.0-0-i-3.5-3-12.5a & $\begin{array}{l}\text { A } \\
B\end{array}$ & - & - & - & 0.375 & $\begin{array}{c}3.5 \\
(1.75)\end{array}$ & 0.44 & 2 & 1.58 & A2 \\
\hline & $8-5 g-T 4.0-5 \# 3-i-2.5-3-9.5^{a}$ & $\begin{array}{l}\text { A } \\
B\end{array}$ & 10 & $\begin{array}{c}3 \\
(1.5) \\
\end{array}$ & 0.66 & 0.375 & $\begin{array}{c}3.5 \\
(1.75) \\
\end{array}$ & 0.44 & 2 & 1.58 & A2 \\
\hline & $8-5 g-T 4.0-5 \# 3-i-3.5-3-9.5^{\mathrm{a}}$ & $\begin{array}{l}\mathrm{A} \\
\mathrm{B}\end{array}$ & 10 & $\begin{array}{c}3 \\
(1.5) \\
\end{array}$ & 0.66 & 0.375 & $\begin{array}{c}3.5 \\
(1.75) \\
\end{array}$ & 0.44 & 2 & 1.58 & A2 \\
\hline & 8-5g-T4.0-4\#4-i-2.5-3-9.5 & $\begin{array}{l}\mathrm{A} \\
\mathrm{B}\end{array}$ & 8 & $\begin{array}{c}4 \\
(2) \\
\end{array}$ & 0.80 & 0.5 & $\begin{array}{c}3.5 \\
(1.75) \\
\end{array}$ & 0.80 & 2 & 1.58 & A17 \\
\hline & 8-5g-T4.0-4\#4-i-3.5-3-9.5 & $\begin{array}{l}\mathrm{A} \\
\mathrm{B}\end{array}$ & 8 & $\begin{array}{c}4 \\
(2) \\
\end{array}$ & 0.80 & 0.5 & $\begin{array}{c}3.5 \\
(1.75) \\
\end{array}$ & 0.80 & 2 & 1.58 & A17 \\
\hline \multirow{6}{*}{ Group 3} & $8-5-T 4.0-0-i-2.5-3-12.5^{a}$ & $\begin{array}{l}\mathrm{A} \\
\mathrm{B}\end{array}$ & - & - & - & 0.375 & $\begin{array}{c}3.5 \\
(1.75) \\
\end{array}$ & 0.44 & 2 & 1.58 & A2 \\
\hline & $8-5-T 4.0-0-i-3.5-3-12.5^{a}$ & $\begin{array}{l}\text { A } \\
B\end{array}$ & - & - & - & 0.375 & $\begin{array}{c}3.5 \\
(1.75) \\
\end{array}$ & 0.44 & 2 & 1.58 & A2 \\
\hline & $8-5-T 4.0-5 \# 3-i-2.5-3-9.5^{a}$ & $\begin{array}{l}\mathrm{A} \\
\mathrm{B}\end{array}$ & 10 & $\begin{array}{c}3 \\
(1.5) \\
\end{array}$ & 0.66 & 0.375 & $\begin{array}{c}3.5 \\
(1.75) \\
\end{array}$ & 0.44 & 2 & 1.58 & A2 \\
\hline & 8-5-T4.0-5\#3-i-3.5-3-9.5 & $\begin{array}{l}A \\
B\end{array}$ & 10 & $\begin{array}{c}3 \\
(1.5)\end{array}$ & 0.66 & 0.375 & $\begin{array}{c}3.5 \\
(1.75)\end{array}$ & 0.44 & 2 & 1.58 & A2 \\
\hline & $8-5-T 4.0-4 \# 4-i-2.5-3-9.5^{a}$ & $\begin{array}{l}\mathrm{A} \\
\mathrm{B} \\
\end{array}$ & 8 & $\begin{array}{c}4 \\
(2) \\
\end{array}$ & 0.80 & 0.5 & $\begin{array}{c}3.5 \\
(1.75) \\
\end{array}$ & 0.80 & 2 & 1.58 & A17 \\
\hline & $8-5-T 4.0-4 \# 4-i-3.5-3-9.5^{a}$ & $\begin{array}{l}\text { A } \\
B\end{array}$ & 8 & $\begin{array}{c}4 \\
(2) \\
\end{array}$ & 0.80 & 0.5 & $\begin{array}{c}3.5 \\
(1.75) \\
\end{array}$ & 0.80 & 2 & 1.58 & A17 \\
\hline \multirow{3}{*}{ Group 4} & 8-8-F4.1-0-i-2.5-3-10.5 & $\begin{array}{l}\text { A } \\
B\end{array}$ & - & - & - & 0.375 & $\begin{array}{c}4 \\
(2) \\
\end{array}$ & 0.44 & 2 & 1.58 & A1 \\
\hline & 8-8-F4.1-2\#3-i-2.5-3-10 & $\begin{array}{l}\text { A } \\
\text { B }\end{array}$ & 4 & $\begin{array}{c}5 \\
(5.5) \\
\end{array}$ & 0.22 & 0.375 & $\begin{array}{c}4 \\
(2) \\
\end{array}$ & 0.44 & 2 & 1.58 & $\mathrm{~A} 1$ \\
\hline & (3@3)8-8-F4.1-0-i-2.5-3-10.5 & $\begin{array}{l}\text { A } \\
B \\
C\end{array}$ & - & - & - & 0.375 & $\begin{array}{c}3.5 \\
(1.75)\end{array}$ & 0.44 & 3 & 2.37 & A3 \\
\hline
\end{tabular}

a Specimen contained crossties within joint region

b Specimen had no confining reinforcement above joint region

c Value in parenthesis is the spacing between the first hoop and the center of the headed bar 
Table A.2 Cont. Comprehensive test results and data for beam-column joint specimens

\begin{tabular}{|c|c|c|c|c|c|c|c|c|c|c|}
\hline & Specimen & Head & $\begin{array}{c}\text { Failure } \\
\text { Type }\end{array}$ & $\begin{array}{c}\text { Lead (Head) } \\
\text { Slip } \\
\text { in. }\end{array}$ & $\begin{array}{l}\boldsymbol{T}_{\max } \\
\text { kips }\end{array}$ & $\begin{array}{c}\boldsymbol{f}_{\text {su,max }} \\
\mathrm{ksi}\end{array}$ & $\begin{array}{l}\boldsymbol{T}_{\text {ind }} \\
\text { kips }\end{array}$ & $\begin{array}{l}\boldsymbol{T}_{\text {total }} \\
\text { kips }\end{array}$ & $\begin{array}{c}\boldsymbol{T} \\
\text { kips }\end{array}$ & $\begin{array}{l}f_{s u} \\
\mathrm{ksi}\end{array}$ \\
\hline \multirow{6}{*}{ Group 1} & $8-5-T 4.0-0-i-3-3-15.5^{a b}$ & $\begin{array}{l}\mathrm{A} \\
\mathrm{B}\end{array}$ & $\mathrm{SB} / \mathrm{FP}$ & $\begin{array}{c}0.019 \\
-\end{array}$ & $\begin{array}{l}81.6 \\
92.2\end{array}$ & $\begin{array}{l}103.3 \\
116.7\end{array}$ & $\begin{array}{l}81.6 \\
79.3\end{array}$ & 160.9 & 80.4 & 101.8 \\
\hline & $8-5-T 4.0-0-\mathrm{i}-4-3-15.5^{\mathrm{a}} \mathrm{b}$ & $\begin{array}{l}\text { A } \\
\text { B }\end{array}$ & $\mathrm{SB} / \mathrm{FP}$ & $\begin{array}{c}0.308 \\
-\end{array}$ & $\begin{array}{l}92.5 \\
98.5\end{array}$ & $\begin{array}{l}117.1 \\
124.7\end{array}$ & $\begin{array}{l}92.5 \\
98.4\end{array}$ & 190.9 & 95.4 & 120.8 \\
\hline & $8-5-T 4.0-4 \# 3-i-3-3-12.5^{\text {а с }}$ & $\begin{array}{l}\text { A } \\
\text { B }\end{array}$ & $\mathrm{SB} / \mathrm{FP}$ & $\begin{array}{c}0.227 \\
-\end{array}$ & $\begin{array}{c}87.9 \\
-^{\ddagger}\end{array}$ & $\begin{array}{c}111.3 \\
-^{\ddagger}\end{array}$ & $\begin{array}{l}87.9 \\
87.1\end{array}$ & 175.0 & 87.5 & 110.8 \\
\hline & $8-5-T 4.0-4 \# 3-\mathrm{i}-4-3-12.5^{\mathrm{a}}$ & $\begin{array}{l}\mathrm{A} \\
\mathrm{B}\end{array}$ & $\mathrm{SB} / \mathrm{FP}$ & $\begin{array}{c}- \\
0.239 \\
\end{array}$ & $\begin{array}{c}110.8 \\
95.2 \\
\end{array}$ & $\begin{array}{l}140.3 \\
120.5 \\
\end{array}$ & $\begin{array}{l}97.2 \\
95.1 \\
\end{array}$ & 192.3 & 96.2 & 121.7 \\
\hline & $8-5-T 4.0-4 \# 4-i-3-3-12.5^{a}$ & $\begin{array}{l}\mathrm{A} \\
\mathrm{B}\end{array}$ & $\mathrm{SB} / \mathrm{FP}$ & $\begin{array}{c}0.049 \\
-\end{array}$ & $\begin{array}{l}109.5 \\
111.0\end{array}$ & $\begin{array}{l}138.6 \\
140.5\end{array}$ & $\begin{array}{l}109.4 \\
108.6\end{array}$ & 218.1 & 109.0 & 138.0 \\
\hline & $8-5-T 4.0-4 \# 4-\mathrm{i}-4-3-12.5^{\mathrm{a}}$ & $\begin{array}{l}\text { A } \\
B\end{array}$ & $\mathrm{SB} / \mathrm{FP}$ & $\begin{array}{l}0.228 \\
0.350\end{array}$ & $\begin{array}{l}102.5 \\
103.4\end{array}$ & $\begin{array}{l}129.7 \\
130.9\end{array}$ & $\begin{array}{l}102.5 \\
100.5\end{array}$ & 203.0 & 101.5 & 128.5 \\
\hline \multirow{6}{*}{ Group 2} & $8-5 g-T 4.0-0-i-2.5-3-12.5^{a}$ & $\begin{array}{l}\text { A } \\
\text { B }\end{array}$ & $\mathrm{SB} / \mathrm{FP}$ & $\begin{array}{c}- \\
0.022(0.008)\end{array}$ & $\begin{array}{c}117.6 \\
96.1\end{array}$ & $\begin{array}{l}148.9 \\
121.6\end{array}$ & $\begin{array}{l}99.3 \\
96.1\end{array}$ & 195.4 & 97.7 & 123.7 \\
\hline & $8-5 g-T 4.0-0-i-3.5-3-12.5^{a}$ & $\begin{array}{l}\mathrm{A} \\
\mathrm{B}\end{array}$ & $\mathrm{SB} / \mathrm{FP}$ & $\begin{array}{c}0.427 \\
(0.025)\end{array}$ & $\begin{array}{c}104.6 \\
93.6 \\
\end{array}$ & $\begin{array}{l}132.4 \\
118.5 \\
\end{array}$ & $\begin{array}{l}93.2 \\
93.6 \\
\end{array}$ & 186.8 & 93.4 & 118.2 \\
\hline & $8-5 g-T 4.0-5 \# 3-i-2.5-3-9.5^{a}$ & $\begin{array}{l}\text { A } \\
\text { B }\end{array}$ & SB & $\begin{array}{l}0.190 \\
0.545\end{array}$ & $\begin{array}{l}78.9 \\
92.6\end{array}$ & $\begin{array}{c}99.9 \\
117.2\end{array}$ & $\begin{array}{l}78.9 \\
78.5\end{array}$ & 157.4 & 78.7 & 99.6 \\
\hline & $8-5 g-T 4.0-5 \# 3-i-3.5-3-9.5^{a}$ & $\begin{array}{l}\mathrm{A} \\
\mathrm{B}\end{array}$ & SB & $\begin{array}{l}0.599 \\
0.193\end{array}$ & $\begin{array}{l}88.4 \\
78.7\end{array}$ & $\begin{array}{c}111.9 \\
99.6\end{array}$ & $\begin{array}{l}80.3 \\
78.7\end{array}$ & 159.0 & 79.5 & 100.6 \\
\hline & $8-5 g-T 4.0-4 \# 4-i-2.5-3-9.5^{a}$ & $\begin{array}{l}\text { A } \\
\text { B }\end{array}$ & SB & $\begin{array}{c}0.187 \\
0.498(0.032)\end{array}$ & $\begin{array}{c}92.2 \\
102.2\end{array}$ & $\begin{array}{l}116.7 \\
129.4\end{array}$ & $\begin{array}{l}92.2 \\
89.3\end{array}$ & 181.5 & 90.7 & 114.8 \\
\hline & $8-5 g-T 4.0-4 \# 4-i-3.5-3-9.5^{a}$ & $\begin{array}{l}\mathrm{A} \\
\mathrm{B}\end{array}$ & SB & $\begin{array}{c}- \\
(0.056)\end{array}$ & $\begin{array}{c}112.0 \\
95.8 \\
\end{array}$ & $\begin{array}{l}141.8 \\
121.3 \\
\end{array}$ & $\begin{array}{l}97.6 \\
95.8 \\
\end{array}$ & 193.4 & 96.7 & 122.4 \\
\hline \multirow{6}{*}{ Group 3} & $8-5-T 4.0-0-\mathrm{i}-2.5-3-12.5^{\mathrm{a}}$ & $\begin{array}{l}\mathrm{A} \\
\mathrm{B}\end{array}$ & $\mathrm{SB} / \mathrm{FP}$ & - & $\begin{array}{l}84.0 \\
95.0\end{array}$ & $\begin{array}{l}106.3 \\
120.3\end{array}$ & $\begin{array}{l}84.0 \\
82.6\end{array}$ & 166.6 & 83.3 & 105.4 \\
\hline & $8-5-T 4.0-0-i-3.5-3-12.5^{a}$ & $\begin{array}{l}\mathrm{A} \\
\mathrm{B}\end{array}$ & $\mathrm{SB} / \mathrm{FP}$ & $\begin{array}{c}0.013 \\
- \\
\end{array}$ & $\begin{array}{c}92.1 \\
100.1 \\
\end{array}$ & $\begin{array}{l}116.6 \\
126.7 \\
\end{array}$ & $\begin{array}{l}92.1 \\
91.6 \\
\end{array}$ & 183.7 & 91.9 & 116.3 \\
\hline & $8-5-T 4.0-5 \# 3-i-2.5-3-9.5^{a}$ & $\begin{array}{l}\text { A } \\
B\end{array}$ & SB & $\begin{array}{l}0.185 \\
0.163\end{array}$ & $\begin{array}{l}74.5 \\
74.0\end{array}$ & $\begin{array}{l}94.3 \\
93.7\end{array}$ & $\begin{array}{l}74.5 \\
74.0\end{array}$ & 148.5 & 74.2 & 93.9 \\
\hline & $8-5-T 4.0-5 \# 3-i-3.5-3-9.5^{a}$ & $\begin{array}{l}\mathrm{A} \\
\mathrm{B}\end{array}$ & $\mathrm{SB} / \mathrm{FP}$ & $\begin{array}{c}- \\
0.570 \\
\end{array}$ & $\begin{array}{l}80.7 \\
96.1 \\
\end{array}$ & $\begin{array}{l}102.2 \\
121.6 \\
\end{array}$ & $\begin{array}{l}80.7 \\
80.4 \\
\end{array}$ & 161.1 & 80.6 & 102.0 \\
\hline & $8-5-T 4.0-4 \# 4-i-2.5-3-9.5^{a}$ & $\begin{array}{l}\text { A } \\
B\end{array}$ & $\mathrm{SB} / \mathrm{FP}$ & $\begin{array}{c}- \\
(0.005)\end{array}$ & $\begin{array}{l}94.9 \\
89.5\end{array}$ & $\begin{array}{l}120.1 \\
113.3 \\
\end{array}$ & $\begin{array}{l}91.6 \\
89.5\end{array}$ & 181.1 & 90.5 & 114.6 \\
\hline & 8-5-T4.0-4\#4-i-3.5-3-9.5 & $\begin{array}{l}\mathrm{A} \\
\mathrm{B}\end{array}$ & $\mathrm{SB} / \mathrm{FP}$ & $\begin{array}{c}0.186 \\
- \\
\end{array}$ & $\begin{array}{l}86.6 \\
89.0 \\
\end{array}$ & $\begin{array}{l}109.6 \\
112.7 \\
\end{array}$ & $\begin{array}{l}86.6 \\
84.6 \\
\end{array}$ & 171.1 & 85.6 & 108.4 \\
\hline \multirow{3}{*}{ Group 4} & 8-8-F4.1-0-i-2.5-3-10.5 & $\begin{array}{l}\mathrm{A} \\
\mathrm{B}\end{array}$ & CB & $\begin{array}{l}- \\
-\end{array}$ & $\begin{array}{l}77.8 \\
76.3 \\
\end{array}$ & $\begin{array}{l}98.5 \\
96.6 \\
\end{array}$ & $\begin{array}{l}77.8 \\
76.3 \\
\end{array}$ & 154.1 & 77.1 & 97.6 \\
\hline & 8-8-F4.1-2\#3-i-2.5-3-10 & $\begin{array}{l}\mathrm{A} \\
\mathrm{B}\end{array}$ & CB & $\begin{array}{l}0.107 \\
0.168 \\
\end{array}$ & $\begin{array}{l}73.5 \\
73.3 \\
\end{array}$ & $\begin{array}{l}93.0 \\
92.8 \\
\end{array}$ & $\begin{array}{l}73.5 \\
73.3 \\
\end{array}$ & 146.8 & 73.4 & 92.9 \\
\hline & (3@3)8-8-F4.1-0-i-2.5-3-10.5 & $\begin{array}{l}\text { A } \\
\text { B } \\
\text { C }\end{array}$ & CB & $\begin{array}{l}0.170 \\
0.212 \\
0.162 \\
\end{array}$ & $\begin{array}{l}49.0 \\
56.2 \\
59.1 \\
\end{array}$ & $\begin{array}{l}62.0 \\
71.1 \\
74.8 \\
\end{array}$ & $\begin{array}{l}49.0 \\
56.2 \\
59.1 \\
\end{array}$ & 164.3 & 54.8 & 69.4 \\
\hline
\end{tabular}

a Specimen contained crossties within joint region

b Specimen had no confining reinforcement above joint region

${ }^{\ddagger}$ No anchorage failure on the bar 
Table A.2 Cont. Comprehensive test results and data for beam-column joint specimens

\begin{tabular}{|c|c|c|c|c|c|c|c|c|c|c|}
\hline & Specimen & Head & $\begin{array}{l}c_{o} \\
\text { in. }\end{array}$ & $A_{b r g}$ & $\begin{array}{l}\ell_{e h} \\
\text { in. }\end{array}$ & $\begin{array}{c}\ell_{e h, a v g} \\
\text { in. }\end{array}$ & $\begin{array}{l}\boldsymbol{f}_{\boldsymbol{c m}} \\
\mathrm{psi}\end{array}$ & $\begin{array}{l}\text { Age } \\
\text { days }\end{array}$ & $\begin{array}{l}d_{b} \\
\text { in. }\end{array}$ & $\begin{array}{l}\boldsymbol{A}_{\boldsymbol{b}} \\
\text { in. }^{2}\end{array}$ \\
\hline \multirow{10}{*}{ Group 4} & (3@3)8-8-F4.1-0-i-2.5-3-10.5-HP & $\begin{array}{l}\text { A } \\
\text { B } \\
\text { C }\end{array}$ & 2.0 & $4.1 A_{b}$ & $\begin{array}{l}10.13 \\
10.13 \\
10.75\end{array}$ & 10.33 & 8450 & 9 & 1 & 0.79 \\
\hline & (3@3)8-8-F4.1-2\#3-i-2.5-3-10 & $\begin{array}{l}\text { A } \\
\text { B } \\
\text { C } \\
\end{array}$ & 2.0 & $4.1 A_{b}$ & $\begin{array}{l}10.13 \\
10.00 \\
10.13 \\
\end{array}$ & 10.08 & 8260 & 8 & 1 & 0.79 \\
\hline & (3@3)8-8-F4.1-2\#3-i-2.5-3-10-HP & $\begin{array}{l}\text { A } \\
B \\
\text { C }\end{array}$ & 2.0 & $4.1 A_{b}$ & $\begin{array}{l}10.25 \\
10.13 \\
10.50\end{array}$ & 10.29 & 8260 & 8 & 1 & 0.79 \\
\hline & (3@4)8-8-F4.1-0-i-2.5-3-10.5 & $\begin{array}{l}\text { A } \\
\text { B } \\
\text { C }\end{array}$ & 2.0 & $4.1 A_{b}$ & $\begin{array}{l}10.88 \\
10.75 \\
10.88\end{array}$ & 10.83 & 8450 & 9 & 1 & 0.79 \\
\hline & (3@4)8-8-F4.1-2\#3-i-2.5-3-10 & $\begin{array}{l}\text { A } \\
\text { B } \\
\text { C }\end{array}$ & 2.0 & $4.1 A_{b}$ & $\begin{array}{c}9.75 \\
9.63 \\
10.25\end{array}$ & 9.88 & 8050 & 7 & 1 & 0.79 \\
\hline & (3@4)8-8-F4.1-2\#3-i-2.5-3-10-HP & $\begin{array}{l}\text { A } \\
\text { B } \\
\text { C }\end{array}$ & 2.0 & $4.1 A_{b}$ & $\begin{array}{l}10.00 \\
10.75 \\
10.25\end{array}$ & 10.33 & 8050 & 7 & 1 & 0.79 \\
\hline & (3@5)8-8-F4.1-0-i-2.5-3-10.5 & $\begin{array}{l}\text { A } \\
\text { B } \\
\text { C }\end{array}$ & 2.0 & $4.1 A_{b}$ & $\begin{array}{l}10.50 \\
10.38 \\
10.19\end{array}$ & 10.35 & 8050 & 7 & 1 & 0.79 \\
\hline & (3@5)8-8-F4.1-0-i-2.5-3-10.5-HP & $\begin{array}{l}\text { A } \\
\text { B } \\
\text { C }\end{array}$ & 2.0 & $4.1 A_{b}$ & $\begin{array}{c}9.75 \\
10.50 \\
10.50\end{array}$ & 10.25 & 8260 & 8 & 1 & 0.79 \\
\hline & (3@5)8-8-F4.1-2\#3-i-2.5-3-10.5 & $\begin{array}{l}\text { A } \\
\text { B } \\
\text { C }\end{array}$ & 2.0 & $4.1 A_{b}$ & $\begin{array}{c}9.63 \\
9.75 \\
10.00 \\
\end{array}$ & 9.79 & 8260 & 8 & 1 & 0.79 \\
\hline & (3@5)8-8-F4.1-2\#3-i-2.5-3-10.5-HP & $\begin{array}{l}\text { A } \\
B \\
\text { C }\end{array}$ & 2.0 & $4.1 A_{b}$ & $\begin{array}{c}9.88 \\
10.00 \\
10.13\end{array}$ & 10.00 & 8260 & 8 & 1 & 0.79 \\
\hline \multirow{5}{*}{ Group 5} & 8-12-F4.1-0-i-2.5-3-10 & $\begin{array}{l}\text { A } \\
\text { B }\end{array}$ & 2.0 & $4.1 A_{b}$ & $\begin{array}{l}9.63 \\
9.75\end{array}$ & 9.69 & 11760 & 34 & 1 & 0.79 \\
\hline & 8-12-F4.1-5\#3-i-2.5-3-10 & $\begin{array}{l}\text { A } \\
\text { B }\end{array}$ & 2.0 & $4.1 A_{b}$ & $\begin{array}{l}10.00 \\
10.00\end{array}$ & 10.00 & 11760 & 34 & 1 & 0.79 \\
\hline & (3@3)8-12-F4.1-0-i-2.5-3-10 & $\begin{array}{l}\text { A } \\
\text { B } \\
\text { C }\end{array}$ & 2.0 & $4.1 A_{b}$ & $\begin{array}{c}9.81 \\
10.00 \\
9.88\end{array}$ & 9.90 & 11040 & 31 & 1 & 0.79 \\
\hline & (3@3)8-12-F4.1-5\#3-i-2.5-3-10 & $\begin{array}{l}\text { A } \\
\text { B } \\
\text { C }\end{array}$ & 2.0 & $4.1 A_{b}$ & $\begin{array}{c}10.00 \\
10.13 \\
9.88 \\
\end{array}$ & 10.00 & 11040 & 31 & 1 & 0.79 \\
\hline & (3@4)8-12-F4.1-0-i-2.5-3-10 & $\begin{array}{l}\text { A } \\
\text { B } \\
\text { C }\end{array}$ & 2.0 & $4.1 A_{b}$ & $\begin{array}{c}10.00 \\
9.75 \\
10.00\end{array}$ & 9.92 & 11440 & 32 & 1 & 0.79 \\
\hline
\end{tabular}


Table A.2 Cont. Comprehensive test results and data for beam-column joint specimens

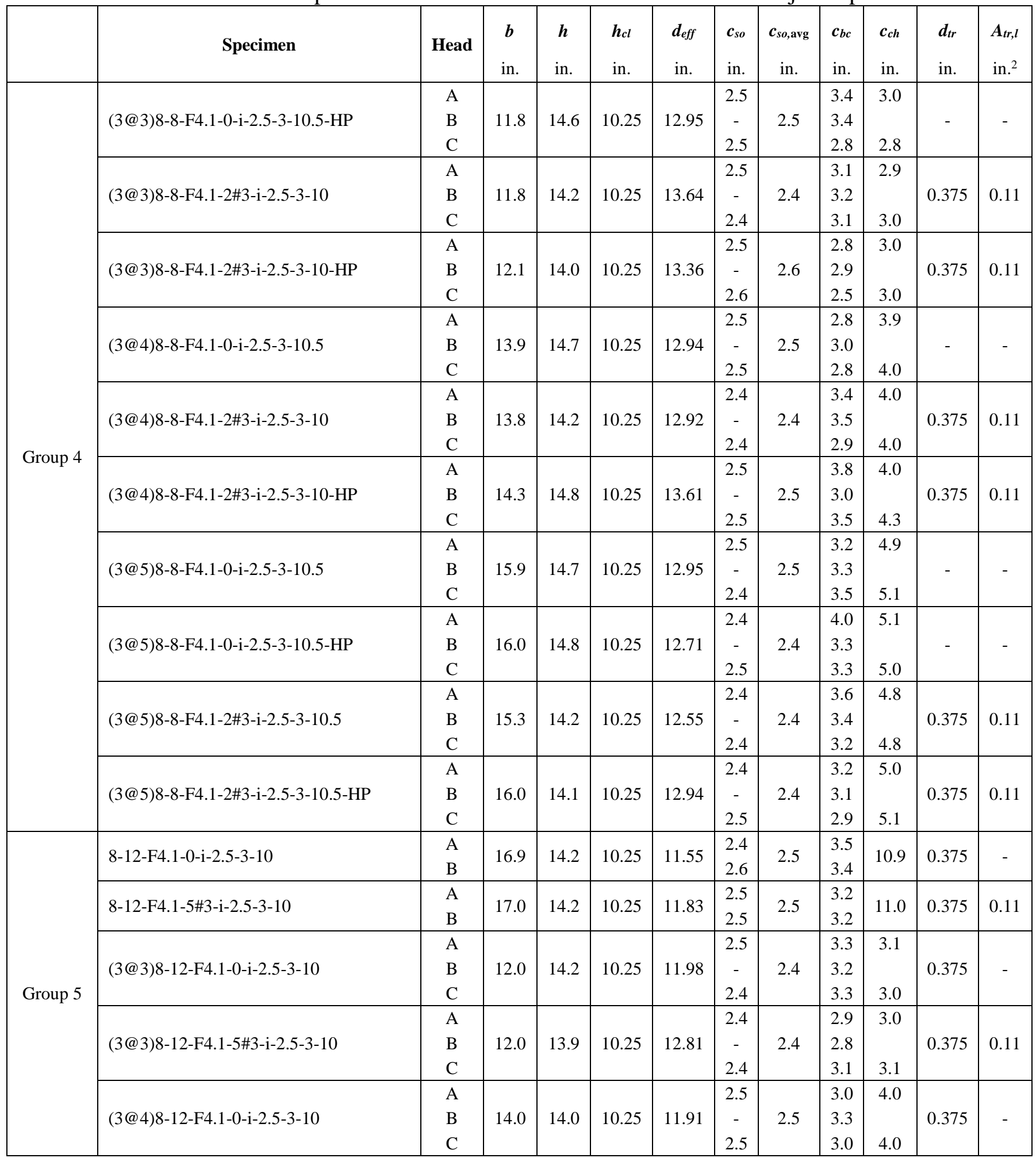


Table A.2 Cont. Comprehensive test results and data for beam-column joint specimens

\begin{tabular}{|c|c|c|c|c|c|c|c|c|c|c|c|}
\hline & Specimen & Head & $N$ & $\begin{array}{l}s_{t r}^{*} \\
\text { in. }\end{array}$ & $\begin{array}{l}\boldsymbol{A}_{\boldsymbol{t t}} \\
\text { in. }^{2}\end{array}$ & $\begin{array}{l}\boldsymbol{d}_{\text {tro }} \\
\text { in. }\end{array}$ & $\begin{array}{l}\text { Stro }^{*} \\
\text { in. }\end{array}$ & $\begin{array}{l}\boldsymbol{A}_{\boldsymbol{a} \boldsymbol{b}} \\
\text { in. }^{2}\end{array}$ & $n$ & $\begin{array}{l}A_{h s} \\
\text { in. }^{2}\end{array}$ & $\begin{array}{l}\text { Long. } \\
\text { Reinf. } \\
\text { Layout }\end{array}$ \\
\hline \multirow{10}{*}{ Group 4} & (3@3)8-8-F4.1-0-i-2.5-3-10.5-HP & $\begin{array}{l}\mathrm{A} \\
\mathrm{B} \\
\mathrm{C}\end{array}$ & - & - & - & 0.375 & $\begin{array}{c}3.5 \\
(1.75)\end{array}$ & 0.44 & 3 & 2.37 & A3 \\
\hline & (3@3)8-8-F4.1-2\#3-i-2.5-3-10 & $\begin{array}{l}\mathrm{A} \\
\mathrm{B} \\
\mathrm{C}\end{array}$ & 4 & $\begin{array}{c}5 \\
(5.5)\end{array}$ & 0.22 & 0.375 & $\begin{array}{c}3 \\
(1.5)\end{array}$ & 0.66 & 3 & 2.37 & A3 \\
\hline & (3@3)8-8-F4.1-2\#3-i-2.5-3-10-HP & $\begin{array}{l}\mathrm{A} \\
\mathrm{B} \\
\mathrm{C} \\
\end{array}$ & 4 & $\begin{array}{c}5 \\
(5.5)\end{array}$ & 0.22 & 0.375 & $\begin{array}{c}3 \\
(1.5)\end{array}$ & 0.66 & 3 & 2.37 & A3 \\
\hline & (3@4)8-8-F4.1-0-i-2.5-3-10.5 & $\begin{array}{l}\text { A } \\
B \\
C\end{array}$ & - & - & - & 0.375 & $\begin{array}{c}4 \\
(2)\end{array}$ & 0.44 & 3 & 2.37 & A3 \\
\hline & (3@4)8-8-F4.1-2\#3-i-2.5-3-10 & $\begin{array}{l}\mathrm{A} \\
\mathrm{B} \\
\mathrm{C}\end{array}$ & 4 & $\begin{array}{c}5 \\
(5.5)\end{array}$ & 0.22 & 0.375 & $\begin{array}{c}3.5 \\
(1.75)\end{array}$ & 0.44 & 3 & 2.37 & A3 \\
\hline & (3@4)8-8-F4.1-2\#3-i-2.5-3-10-HP & $\begin{array}{l}\mathrm{A} \\
\mathrm{B} \\
\mathrm{C}\end{array}$ & 4 & $\begin{array}{c}5 \\
(5.5)\end{array}$ & 0.22 & 0.375 & $\begin{array}{c}3.5 \\
(1.75)\end{array}$ & 0.44 & 3 & 2.37 & A3 \\
\hline & (3@5)8-8-F4.1-0-i-2.5-3-10.5 & $\begin{array}{l}\text { A } \\
B \\
C\end{array}$ & - & - & - & 0.375 & $\begin{array}{c}4 \\
(2)\end{array}$ & 0.44 & 3 & 2.37 & A3 \\
\hline & (3@5)8-8-F4.1-0-i-2.5-3-10.5-HP & $\begin{array}{l}\mathrm{A} \\
\mathrm{B} \\
\mathrm{C} \\
\end{array}$ & - & - & - & 0.375 & $\begin{array}{c}4 \\
(2)\end{array}$ & 0.44 & 3 & 2.37 & A3 \\
\hline & (3@5)8-8-F4.1-2\#3-i-2.5-3-10.5 & $\begin{array}{l}\text { A } \\
\text { B } \\
\text { C }\end{array}$ & 4 & $\begin{array}{c}5 \\
(5.5)\end{array}$ & 0.22 & 0.375 & $\begin{array}{c}4 \\
(2)\end{array}$ & 0.44 & 3 & 2.37 & A3 \\
\hline & (3@5)8-8-F4.1-2\#3-i-2.5-3-10.5-HP & $\begin{array}{l}\mathrm{A} \\
\mathrm{B} \\
\mathrm{C}\end{array}$ & 4 & $\begin{array}{c}5 \\
(5.5)\end{array}$ & 0.22 & 0.375 & $\begin{array}{c}4 \\
(2)\end{array}$ & 0.44 & 3 & 2.37 & A3 \\
\hline \multirow{5}{*}{ Group 5} & 8-12-F4.1-0-i-2.5-3-10 & $\begin{array}{l}\mathrm{A} \\
\mathrm{B} \\
\end{array}$ & - & - & - & 0.375 & $\begin{array}{c}4 \\
(2) \\
\end{array}$ & 0.44 & 2 & 1.58 & A4 \\
\hline & 8-12-F4.1-5\#3-i-2.5-3-10 & $\begin{array}{l}\text { A } \\
\text { B }\end{array}$ & 10 & $\begin{array}{c}3 \\
(1.5) \\
\end{array}$ & 0.66 & 0.375 & $\begin{array}{c}4 \\
(2) \\
\end{array}$ & 0.44 & 2 & 1.58 & A5 \\
\hline & (3@3)8-12-F4.1-0-i-2.5-3-10 & $\begin{array}{l}\mathrm{A} \\
\mathrm{B} \\
\mathrm{C}\end{array}$ & - & - & - & 0.375 & $\begin{array}{c}3 \\
(1.5)\end{array}$ & 0.66 & 3 & 2.37 & A5 \\
\hline & (3@3)8-12-F4.1-5\#3-i-2.5-3-10 & $\begin{array}{l}\mathrm{A} \\
\mathrm{B} \\
\mathrm{C}\end{array}$ & 10 & $\begin{array}{c}3 \\
(1.5)\end{array}$ & 0.66 & 0.375 & $\begin{array}{c}3 \\
(1.5)\end{array}$ & 0.66 & 3 & 2.37 & A5 \\
\hline & (3@4)8-12-F4.1-0-i-2.5-3-10 & $\begin{array}{l}\text { A } \\
B \\
C\end{array}$ & - & - & - & 0.375 & $\begin{array}{c}3 \\
(1.5)\end{array}$ & 0.66 & 3 & 2.37 & A5 \\
\hline
\end{tabular}

\footnotetext{
${ }^{*}$ Value in parenthesis is the spacing between the first hoop and the center of the headed bar
} 
Table A.2 Cont. Comprehensive test results and data for beam-column joint specimens

\begin{tabular}{|c|c|c|c|c|c|c|c|c|c|c|}
\hline & Specimen & Head & $\begin{array}{l}\text { Failure } \\
\text { Type }\end{array}$ & $\begin{array}{l}\text { Lead (Head) } \\
\text { Slip } \\
\text { in. }\end{array}$ & $\begin{array}{l}\boldsymbol{T}_{\max } \\
\text { kips }\end{array}$ & $\begin{array}{c}\boldsymbol{f}_{\text {su, max }} \\
\text { ksi }\end{array}$ & $\begin{array}{l}\boldsymbol{T}_{\text {ind }} \\
\text { kips }\end{array}$ & $\begin{array}{l}\boldsymbol{T}_{\text {total }} \\
\text { kips }\end{array}$ & $\begin{array}{c}\boldsymbol{T} \\
\text { kips }\end{array}$ & $\begin{array}{l}f_{s u} \\
\text { ksi }\end{array}$ \\
\hline \multirow{10}{*}{ Group 4} & (3@3)8-8-F4.1-0-i-2.5-3-10.5-HP & $\begin{array}{l}\text { A } \\
\text { B } \\
\text { C }\end{array}$ & $\mathrm{CB} / \mathrm{FP}$ & $\begin{array}{l}0.399 \\
0.448 \\
0.075\end{array}$ & $\begin{array}{l}55.3 \\
50.2 \\
46.0\end{array}$ & $\begin{array}{l}70.0 \\
63.5 \\
58.2\end{array}$ & $\begin{array}{l}55.3 \\
50.2 \\
46.0\end{array}$ & 151.4 & 50.5 & 63.9 \\
\hline & (3@3)8-8-F4.1-2\#3-i-2.5-3-10 & $\begin{array}{l}\text { A } \\
\text { B } \\
\text { C }\end{array}$ & CB & $\begin{array}{l}0.097 \\
0.202 \\
0.127 \\
\end{array}$ & $\begin{array}{l}53.2 \\
65.3 \\
67.3 \\
\end{array}$ & $\begin{array}{l}67.3 \\
82.7 \\
85.2 \\
\end{array}$ & $\begin{array}{l}53.2 \\
65.3 \\
67.3 \\
\end{array}$ & 185.8 & 61.9 & 78.4 \\
\hline & (3@3)8-8-F4.1-2\#3-i-2.5-3-10-HP & $\begin{array}{l}\text { A } \\
\text { B } \\
\text { C } \\
\end{array}$ & СВ & $\begin{array}{l}0.100 \\
0.150 \\
0.151 \\
\end{array}$ & $\begin{array}{l}51.4 \\
58.7 \\
60.0 \\
\end{array}$ & $\begin{array}{l}65.1 \\
74.3 \\
75.9 \\
\end{array}$ & $\begin{array}{l}51.4 \\
58.7 \\
60.0 \\
\end{array}$ & 170.1 & 56.7 & 71.8 \\
\hline & (3@4)8-8-F4.1-0-i-2.5-3-10.5 & $\begin{array}{l}\text { A } \\
\text { B } \\
\text { C }\end{array}$ & СВ & $\begin{array}{l}0.117 \\
0.339 \\
0.146 \\
\end{array}$ & $\begin{array}{l}62.8 \\
62.3 \\
51.4\end{array}$ & $\begin{array}{l}79.5 \\
78.9 \\
65.1\end{array}$ & $\begin{array}{l}62.8 \\
62.3 \\
51.0\end{array}$ & 176.1 & 58.7 & 74.3 \\
\hline & (3@4)8-8-F4.1-2\#3-i-2.5-3-10 & $\begin{array}{l}\text { A } \\
\text { B } \\
\text { C } \\
\end{array}$ & CB & $\begin{array}{l}0.113 \\
0.213 \\
0.203 \\
\end{array}$ & $\begin{array}{l}61.7 \\
52.9 \\
51.8 \\
\end{array}$ & $\begin{array}{l}78.1 \\
67.0 \\
65.6 \\
\end{array}$ & $\begin{array}{l}61.7 \\
52.9 \\
51.8 \\
\end{array}$ & 166.4 & 55.5 & 70.3 \\
\hline & (3@4)8-8-F4.1-2\#3-i-2.5-3-10-HP & $\begin{array}{l}\text { A } \\
\text { B } \\
\text { C }\end{array}$ & СВ & $\begin{array}{c}0.143 \\
0.338 \\
-\end{array}$ & $\begin{array}{l}70.6 \\
70.2 \\
68.8 \\
\end{array}$ & $\begin{array}{l}89.4 \\
88.9 \\
87.1\end{array}$ & $\begin{array}{l}70.5 \\
70.2 \\
68.8\end{array}$ & 209.5 & 69.8 & 88.4 \\
\hline & (3@5)8-8-F4.1-0-i-2.5-3-10.5 & $\begin{array}{l}\text { A } \\
\text { B } \\
\text { C } \\
\end{array}$ & $\mathrm{CB}$ & $\begin{array}{l}0.255 \\
0.172 \\
0.237 \\
\end{array}$ & $\begin{array}{l}67.9 \\
65.7 \\
58.4 \\
\end{array}$ & $\begin{array}{l}85.9 \\
83.2 \\
73.9 \\
\end{array}$ & $\begin{array}{l}67.9 \\
65.7 \\
58.4 \\
\end{array}$ & 192.0 & 64.0 & 81.0 \\
\hline & (3@5)8-8-F4.1-0-i-2.5-3-10.5-HP & $\begin{array}{l}\text { A } \\
\text { B } \\
\text { C } \\
\end{array}$ & $\mathrm{CB}$ & $\begin{array}{c}0.113 \\
- \\
- \\
\end{array}$ & $\begin{array}{l}62.9 \\
60.8 \\
55.9 \\
\end{array}$ & $\begin{array}{l}79.6 \\
77.0 \\
70.8 \\
\end{array}$ & $\begin{array}{l}62.9 \\
60.8 \\
55.9 \\
\end{array}$ & 179.6 & 59.9 & 75.8 \\
\hline & (3@5)8-8-F4.1-2\#3-i-2.5-3-10.5 & $\begin{array}{l}\text { A } \\
\text { B } \\
\text { C } \\
\end{array}$ & СВ & $\begin{array}{c}- \\
0.388 \\
0.217\end{array}$ & $\begin{array}{l}61.4 \\
56.1 \\
56.7 \\
\end{array}$ & $\begin{array}{l}77.7 \\
71.0 \\
71.8 \\
\end{array}$ & $\begin{array}{l}61.4 \\
50.1 \\
56.7\end{array}$ & 168.2 & 56.1 & 71.0 \\
\hline & (3@5)8-8-F4.1-2\#3-i-2.5-3-10.5-HP & $\begin{array}{l}\text { A } \\
\text { B } \\
\text { C }\end{array}$ & CB & $\begin{array}{l}0.036 \\
0.171 \\
0.168\end{array}$ & $\begin{array}{l}62.0 \\
70.8 \\
63.6\end{array}$ & $\begin{array}{l}78.5 \\
89.6 \\
80.5\end{array}$ & $\begin{array}{l}62.0 \\
70.8 \\
63.6\end{array}$ & 196.4 & 65.5 & 82.9 \\
\hline \multirow{5}{*}{ Group 5} & 8-12-F4.1-0-i-2.5-3-10 & $\begin{array}{l}\text { A } \\
\text { B }\end{array}$ & $\mathrm{CB}$ & $\begin{array}{c}0.110 \\
0.099(0.079)\end{array}$ & $\begin{array}{l}72.5 \\
71.1 \\
\end{array}$ & $\begin{array}{l}91.8 \\
90.0 \\
\end{array}$ & $\begin{array}{l}72.5 \\
71.1 \\
\end{array}$ & 143.6 & 71.8 & 90.9 \\
\hline & 8-12-F4.1-5\#3-i-2.5-3-10 & $\begin{array}{l}\text { A } \\
\text { B }\end{array}$ & SB/FP & $\begin{array}{c}- \\
(0.006)\end{array}$ & $\begin{array}{l}88.4 \\
86.0 \\
\end{array}$ & $\begin{array}{l}111.9 \\
108.9 \\
\end{array}$ & $\begin{array}{l}88.4 \\
86.0 \\
\end{array}$ & 174.3 & 87.2 & 110.4 \\
\hline & (3@3)8-12-F4.1-0-i-2.5-3-10 & $\begin{array}{l}\text { A } \\
\text { B } \\
\text { C }\end{array}$ & $\mathrm{CB}$ & $\begin{array}{l}- \\
- \\
-\end{array}$ & $\begin{array}{l}38.5 \\
42.3 \\
47.7\end{array}$ & $\begin{array}{l}48.7 \\
53.5 \\
60.4\end{array}$ & $\begin{array}{l}38.5 \\
40.3 \\
47.7\end{array}$ & 126.5 & 42.2 & 53.4 \\
\hline & (3@3)8-12-F4.1-5\#3-i-2.5-3-10 & $\begin{array}{l}\text { A } \\
\text { B } \\
\text { C }\end{array}$ & СВ & $\begin{array}{l}0.230 \\
0.252 \\
0.123\end{array}$ & $\begin{array}{l}65.7 \\
63.9 \\
55.1\end{array}$ & $\begin{array}{l}83.2 \\
80.9 \\
69.7\end{array}$ & $\begin{array}{l}65.7 \\
63.9 \\
55.1\end{array}$ & 187.4 & 61.6 & 78.0 \\
\hline & (3@4)8-12-F4.1-0-i-2.5-3-10 & $\begin{array}{l}\text { A } \\
\text { B } \\
\text { C }\end{array}$ & $\mathrm{CB}$ & $\begin{array}{c}0.120 \\
0.069 \\
0.118(0.043)\end{array}$ & $\begin{array}{l}49.1 \\
55.1 \\
42.5\end{array}$ & $\begin{array}{l}62.2 \\
69.7 \\
53.8\end{array}$ & $\begin{array}{l}49.1 \\
55.0 \\
42.5\end{array}$ & 146.6 & 48.9 & 61.9 \\
\hline
\end{tabular}


Table A.2 Cont. Comprehensive test results and data for beam-column joint specimens

\begin{tabular}{|c|c|c|c|c|c|c|c|c|c|c|}
\hline & Specimen & Head & $\begin{array}{l}\boldsymbol{c}_{\boldsymbol{o}} \\
\text { in. }\end{array}$ & $A_{b r g}$ & $\begin{array}{l}\ell_{\text {eh }} \\
\text { in. }\end{array}$ & $\begin{array}{c}\ell \text { eh,avg } \\
\text { in. }\end{array}$ & $\begin{array}{l}\boldsymbol{f}_{\boldsymbol{c m}} \\
\text { psi }\end{array}$ & $\begin{array}{l}\text { Age } \\
\text { days }\end{array}$ & $\begin{array}{l}\boldsymbol{d}_{\boldsymbol{b}} \\
\text { in. }\end{array}$ & $\begin{array}{c}\boldsymbol{A}_{\boldsymbol{b}} \\
\text { in. }^{2}\end{array}$ \\
\hline \multirow{3}{*}{ Group 5} & (3@4)8-12-F4.1-5\#3-i-2.5-3-10 & $\begin{array}{l}\text { A } \\
B \\
C\end{array}$ & 2.0 & $4.1 A_{b}$ & $\begin{array}{l}9.81 \\
9.88 \\
9.63\end{array}$ & 9.77 & 11440 & 32 & 1 & 0.79 \\
\hline & (3@5)8-12-F4.1-0-i-2.5-3-10 & $\begin{array}{l}\text { A } \\
B \\
C\end{array}$ & 2.0 & $4.1 A_{b}$ & $\begin{array}{c}9.88 \\
10.13 \\
9.75\end{array}$ & 9.92 & 11460 & 33 & 1 & 0.79 \\
\hline & (3@5)8-12-F4.1-5\#3-i-2.5-3-10 & $\begin{array}{l}\mathrm{A} \\
\mathrm{B} \\
\mathrm{C} \\
\end{array}$ & 2.0 & $4.1 A_{b}$ & $\begin{array}{l}9.75 \\
9.38 \\
9.69 \\
\end{array}$ & 9.60 & 11460 & 33 & 1 & 0.79 \\
\hline \multirow{12}{*}{ Group 6} & 8-5-S6.5-0-i-2.5-3-11.25 & $\begin{array}{l}\text { A } \\
B\end{array}$ & 1.8 & $6.5 A_{b}$ & $\begin{array}{l}11.00 \\
11.13\end{array}$ & 11.06 & 5500 & 6 & 1 & 0.79 \\
\hline & 8-5-S6.5-0-i-2.5-3-14.25 & $\begin{array}{l}\text { A } \\
B\end{array}$ & 1.8 & $6.5 A_{b}$ & $\begin{array}{l}14.38 \\
14.13\end{array}$ & 14.25 & 5500 & 6 & 1 & 0.79 \\
\hline & $8-5-04.5-0-i-2.5-3-11.25$ & $\begin{array}{l}\text { A } \\
B\end{array}$ & 1.6 & $4.5 A_{b}$ & $\begin{array}{l}11.00 \\
11.50\end{array}$ & 11.25 & 5500 & 6 & 1 & 0.79 \\
\hline & $8-5-04.5-0-i-2.5-3-14.25$ & $\begin{array}{l}\text { A } \\
B\end{array}$ & 1.6 & $4.5 A_{b}$ & $\begin{array}{l}14.38 \\
13.88\end{array}$ & 14.13 & 5500 & 6 & 1 & 0.79 \\
\hline & 8-5-S6.5-2\#3-i-2.5-3-9.25 & $\begin{array}{l}A \\
B\end{array}$ & 1.8 & $6.5 A_{b}$ & $\begin{array}{l}9.25 \\
9.00\end{array}$ & 9.13 & 5750 & 7 & 1 & 0.79 \\
\hline & 8-5-S6.5-2\#3-i-2.5-3-12.25 & $\begin{array}{l}\text { A } \\
B\end{array}$ & 1.8 & $6.5 A_{b}$ & $\begin{array}{l}12.50 \\
12.13\end{array}$ & 12.31 & 5750 & 7 & 1 & 0.79 \\
\hline & $8-5-04.5-2 \# 3-i-2.5-3-9.25$ & $\begin{array}{l}\text { A } \\
B\end{array}$ & 1.6 & $4.5 A_{b}$ & $\begin{array}{l}9.38 \\
9.38\end{array}$ & 9.38 & 5750 & 7 & 1 & 0.79 \\
\hline & 8-5-O4.5-2\#3-i-2.5-3-12.25 & $\begin{array}{l}\text { A } \\
B\end{array}$ & 1.6 & $4.5 A_{b}$ & $\begin{array}{l}12.00 \\
12.00\end{array}$ & 12.00 & 5750 & 7 & 1 & 0.79 \\
\hline & 8-5-S6.5-5\#3-i-2.5-3-8.25 & $\begin{array}{l}A \\
B\end{array}$ & 1.8 & $6.5 A_{b}$ & $\begin{array}{l}8.38 \\
8.25 \\
\end{array}$ & 8.31 & 5900 & 8 & 1 & 0.79 \\
\hline & 8-5-S6.5-5\#3-i-2.5-3-11.25 & $\begin{array}{l}A \\
B\end{array}$ & 1.8 & $6.5 A_{b}$ & $\begin{array}{l}10.88 \\
11.00\end{array}$ & 10.94 & 5900 & 8 & 1 & 0.79 \\
\hline & $8-5-04.5-5 \# 3-i-2.5-3-8.25$ & $\begin{array}{l}A \\
B\end{array}$ & 1.6 & $4.5 A_{b}$ & $\begin{array}{l}8.13 \\
7.88 \\
\end{array}$ & 8.00 & 5900 & 8 & 1 & 0.79 \\
\hline & 8-5-O4.5-5\#3-i-2.5-3-11.25 & $\begin{array}{l}\text { A } \\
B\end{array}$ & 1.6 & $4.5 A_{b}$ & $\begin{array}{l}11.38 \\
10.88 \\
\end{array}$ & 11.13 & 5900 & 8 & 1 & 0.79 \\
\hline \multirow{5}{*}{ Group 7} & 8-5-T9.5-0-i-2.5-3-14.5 & $\begin{array}{l}A \\
B\end{array}$ & 1.4 & $9.5 A_{b}$ & $\begin{array}{l}14.25 \\
14.50\end{array}$ & 14.38 & 4970 & 8 & 1 & 0.79 \\
\hline & 8-5-09.1-0-i-2.5-3-14.5 & $\begin{array}{l}A \\
B\end{array}$ & 1.3 & $9.1 A_{b}$ & $\begin{array}{l}14.38 \\
14.38 \\
\end{array}$ & 14.38 & 4970 & 8 & 1 & 0.79 \\
\hline & 8-5-T9.5-5\#3-i-2.5-3-14.5 & $\begin{array}{l}\text { A } \\
B\end{array}$ & 1.4 & $9.5 A_{b}$ & $\begin{array}{l}14.50 \\
14.25 \\
\end{array}$ & 14.38 & 5420 & 13 & 1 & 0.79 \\
\hline & 8-5-09.1-5\#3-i-2.5-3-14.5 & $\begin{array}{l}A \\
B\end{array}$ & 1.3 & $9.1 A_{b}$ & $\begin{array}{l}14.06 \\
14.13\end{array}$ & 14.09 & 4970 & 8 & 1 & 0.79 \\
\hline & (3@5.5)8-5-T9.5-0-i-2.5-3-14.5 & $\begin{array}{l}\text { A } \\
B \\
C\end{array}$ & 1.4 & $9.5 A_{b}$ & $\begin{array}{l}14.25 \\
14.25 \\
14.25\end{array}$ & 14.25 & 4960 & 9 & 1 & 0.79 \\
\hline
\end{tabular}


Table A.2 Cont. Comprehensive test results and data for beam-column joint specimens

\begin{tabular}{|c|c|c|c|c|c|c|c|c|c|c|c|c|}
\hline & Specimen & Head & $\begin{array}{c}\boldsymbol{b} \\
\text { in. }\end{array}$ & $\begin{array}{c}\boldsymbol{h} \\
\text { in. }\end{array}$ & $\begin{array}{l}\boldsymbol{h}_{c l} \\
\text { in. }\end{array}$ & $\begin{array}{c}\boldsymbol{d}_{\text {eff }} \\
\text { in. }\end{array}$ & $\begin{array}{l}\boldsymbol{c}_{\boldsymbol{s} o} \\
\text { in. }\end{array}$ & $\begin{array}{c}\boldsymbol{C}_{\boldsymbol{s o}, \text { avg }} \\
\text { in. }\end{array}$ & $\begin{array}{l}c_{b c} \\
\text { in. }\end{array}$ & $\begin{array}{l}\boldsymbol{c}_{\boldsymbol{c h}} \\
\text { in. }\end{array}$ & $\begin{array}{l}\boldsymbol{d}_{t r} \\
\text { in. }\end{array}$ & $\begin{array}{c}\boldsymbol{A}_{t r, \boldsymbol{I}} \\
\text { in. }^{2}\end{array}$ \\
\hline \multirow{3}{*}{ Group 5} & (3@4)8-12-F4.1-5\#3-i-2.5-3-10 & $\begin{array}{l}\text { A } \\
B \\
C\end{array}$ & 13.8 & 14.0 & 10.25 & 12.48 & $\begin{array}{c}2.4 \\
- \\
2.5 \\
\end{array}$ & 2.4 & $\begin{array}{l}3.2 \\
3.1 \\
3.3 \\
\end{array}$ & $\begin{array}{l}3.9 \\
4.0 \\
\end{array}$ & 0.375 & 0.11 \\
\hline & (3@5)8-12-F4.1-0-i-2.5-3-10 & $\begin{array}{l}\text { A } \\
B \\
C\end{array}$ & 16.1 & 14.1 & 10.25 & 11.88 & $\begin{array}{c}2.5 \\
- \\
2.5\end{array}$ & 2.5 & $\begin{array}{l}3.2 \\
3.0 \\
3.3\end{array}$ & $\begin{array}{l}5.0 \\
5.1\end{array}$ & 0.375 & - \\
\hline & (3@5)8-12-F4.1-5\#3-i-2.5-3-10 & $\begin{array}{l}\text { A } \\
B \\
C\end{array}$ & 16.3 & 14.3 & 10.25 & 12.31 & $\begin{array}{c}2.5 \\
- \\
2.5 \\
\end{array}$ & 2.5 & $\begin{array}{l}3.5 \\
3.9 \\
3.6 \\
\end{array}$ & $\begin{array}{l}5.1 \\
5.2 \\
\end{array}$ & 0.375 & 0.11 \\
\hline \multirow{7}{*}{ Group 6} & 8-5-S6.5-0-i-2.5-3-11.25 & $\begin{array}{l}\text { A } \\
B\end{array}$ & 16.8 & 16.1 & 10.25 & 12.70 & $\begin{array}{l}2.5 \\
2.5 \\
\end{array}$ & 2.5 & $\begin{array}{l}3.4 \\
3.3 \\
\end{array}$ & 10.8 & 0.375 & - \\
\hline & $8-5-04.5-0-\mathrm{i}-2.5-3-14.25$ & $\begin{array}{l}A \\
B\end{array}$ & 17.0 & 19.1 & 10.25 & 13.01 & $\begin{array}{l}2.5 \\
2.5\end{array}$ & 2.5 & $\begin{array}{l}3.1 \\
3.6\end{array}$ & 11.0 & 0.375 & - \\
\hline & 8-5-S6.5-2\#3-i-2.5-3-9.25 & $\begin{array}{l}A \\
B\end{array}$ & 17.5 & 14.0 & 10.25 & 12.25 & $\begin{array}{l}2.8 \\
2.5\end{array}$ & 2.6 & $\begin{array}{l}3.0 \\
3.3\end{array}$ & 11.3 & 0.375 & 0.11 \\
\hline & 8-5-S6.5-2\#3-i-2.5-3-12.25 & $\begin{array}{l}A \\
B\end{array}$ & 16.9 & 17.1 & 10.25 & 12.96 & $\begin{array}{l}2.4 \\
2.5 \\
\end{array}$ & 2.5 & $\begin{array}{l}2.8 \\
3.2 \\
\end{array}$ & 11.0 & 0.375 & 0.11 \\
\hline & 8-5-O4.5-2\#3-i-2.5-3-9.25 & $\begin{array}{l}A \\
B\end{array}$ & 17.0 & 14.1 & 10.25 & 12.39 & $\begin{array}{l}2.5 \\
2.5 \\
\end{array}$ & 2.5 & $\begin{array}{l}3.1 \\
3.1 \\
\end{array}$ & 11.0 & 0.375 & 0.11 \\
\hline & 8-5-O4.5-2\#3-i-2.5-3-12.25 & $\begin{array}{l}A \\
B\end{array}$ & 16.6 & 16.9 & 10.25 & 12.73 & $\begin{array}{l}2.4 \\
2.5\end{array}$ & 2.4 & $\begin{array}{l}3.3 \\
3.3 \\
\end{array}$ & 10.8 & 0.375 & 0.11 \\
\hline & 8-5-O4.5-5\#3-i-2.5-3-11.25 & $\begin{array}{l}\mathrm{A} \\
\mathrm{B}\end{array}$ & 16.6 & 15.9 & 10.25 & 12.81 & $\begin{array}{l}2.5 \\
2.4 \\
\end{array}$ & 2.4 & $\begin{array}{l}2.9 \\
3.4 \\
\end{array}$ & 10.8 & 0.375 & 0.11 \\
\hline \multirow{5}{*}{ Group 7} & 8-5-T9.5-0-i-2.5-3-14.5 & $\begin{array}{l}\text { A } \\
\text { B }\end{array}$ & 17.0 & 19.1 & 10.25 & 13.43 & $\begin{array}{l}2.6 \\
2.5 \\
\end{array}$ & 2.6 & $\begin{array}{l}3.4 \\
3.1 \\
\end{array}$ & 10.9 & 0.375 & - \\
\hline & 8-5-09.1-0-i-2.5-3-14.5 & $\begin{array}{l}\mathrm{A} \\
\mathrm{B}\end{array}$ & 17.3 & 19.2 & 10.25 & 13.54 & $\begin{array}{l}2.5 \\
2.8 \\
\end{array}$ & 2.6 & $\begin{array}{l}3.2 \\
3.2 \\
\end{array}$ & 11.0 & 0.375 & - \\
\hline & 8-5-T9.5-5\#3-i-2.5-3-14.5 & $\begin{array}{l}\text { A } \\
B\end{array}$ & 17.1 & 19.2 & 10.25 & 14.22 & $\begin{array}{l}2.5 \\
2.6\end{array}$ & 2.6 & $\begin{array}{l}3.2 \\
3.5\end{array}$ & 11.0 & 0.375 & 0.11 \\
\hline & 8-5-09.1-5\#3-i-2.5-3-14.5 & $\begin{array}{l}\mathrm{A} \\
\mathrm{B}\end{array}$ & 17.0 & 19.2 & 10.25 & $-\ddagger$ & $\begin{array}{l}2.5 \\
2.5 \\
\end{array}$ & 2.5 & $\begin{array}{l}3.5 \\
3.4 \\
\end{array}$ & 11.0 & 0.375 & 0.11 \\
\hline & (3@5.5)8-5-T9.5-0-i-2.5-3-14.5 & $\begin{array}{l}\text { A } \\
B \\
C\end{array}$ & 16.9 & 19.2 & 10.25 & 14.08 & $\begin{array}{c}2.4 \\
- \\
2.5\end{array}$ & 2.4 & $\begin{array}{l}3.4 \\
3.4 \\
3.4\end{array}$ & $\begin{array}{l}5.5 \\
5.5\end{array}$ & 0.375 & - \\
\hline
\end{tabular}

\footnotetext{
${ }^{\ddagger} d_{\text {eff }}$ was not calculated for specimen with bar yielding
} 
Table A.2 Cont. Comprehensive test results and data for beam-column joint specimens

\begin{tabular}{|c|c|c|c|c|c|c|c|c|c|c|c|}
\hline & Specimen & Head & $N$ & $\begin{array}{l}\boldsymbol{s}_{t r}{ }^{*} \\
\text { in. }\end{array}$ & $\begin{array}{l}\boldsymbol{A}_{\boldsymbol{t t}} \\
\text { in. }^{2}\end{array}$ & $\begin{array}{l}\boldsymbol{d}_{\text {tro }} \\
\text { in. }\end{array}$ & $\begin{array}{l}S_{\text {tro }}{ }^{*} \\
\text { in. }\end{array}$ & $\begin{array}{l}A_{a b} \\
\text { in. }^{2}\end{array}$ & $n$ & $\begin{array}{l}A_{h s} \\
\text { in. }^{2}\end{array}$ & $\begin{array}{l}\text { Long. } \\
\text { Reinf. } \\
\text { Layout }\end{array}$ \\
\hline \multirow{3}{*}{ Group 5} & (3@4)8-12-F4.1-5\#3-i-2.5-3-10 & $\begin{array}{l}\text { A } \\
B \\
C\end{array}$ & 10 & $\begin{array}{c}3 \\
(1.5)\end{array}$ & 0.66 & 0.375 & $\begin{array}{c}3 \\
(1.5)\end{array}$ & 0.66 & 3 & 2.37 & A5 \\
\hline & (3@5)8-12-F4.1-0-i-2.5-3-10 & $\begin{array}{l}\text { A } \\
B \\
C \\
\end{array}$ & - & - & - & 0.375 & $\begin{array}{c}3 \\
(1.5)\end{array}$ & 0.66 & 3 & 2.37 & A5 \\
\hline & (3@5)8-12-F4.1-5\#3-i-2.5-3-10 & $\begin{array}{l}A \\
B \\
C \\
\end{array}$ & 10 & $\begin{array}{c}3 \\
(1.5)\end{array}$ & 0.66 & 0.375 & $\begin{array}{c}3 \\
(1.5)\end{array}$ & 0.66 & 3 & 2.37 & A5 \\
\hline \multirow{12}{*}{ Group 6} & 8-5-S6.5-0-i-2.5-3-11.25 & $\begin{array}{l}\text { A } \\
\text { B }\end{array}$ & - & - & - & 0.5 & $\begin{array}{c}3.5 \\
(1.75) \\
\end{array}$ & 0.80 & 2 & 1.58 & A4 \\
\hline & 8-5-S6.5-0-i-2.5-3-14.25 & $\begin{array}{l}\mathrm{A} \\
\mathrm{B}\end{array}$ & - & - & - & 0.5 & $\begin{array}{c}3.5 \\
(1.75) \\
\end{array}$ & 0.80 & 2 & 1.58 & A4 \\
\hline & 8-5-04.5-0-i-2.5-3-11.25 & $\begin{array}{l}A \\
B\end{array}$ & - & - & - & 0.5 & $\begin{array}{c}3.5 \\
(1.75)\end{array}$ & 0.80 & 2 & 1.58 & A4 \\
\hline & 8-5-O4.5-0-i-2.5-3-14.25 & $\begin{array}{l}\text { A } \\
B\end{array}$ & - & - & - & 0.5 & $\begin{array}{c}3.5 \\
(1.75) \\
\end{array}$ & 0.80 & 2 & 1.58 & A4 \\
\hline & 8-5-S6.5-2\#3-i-2.5-3-9.25 & $\begin{array}{l}\text { A } \\
\text { B }\end{array}$ & 4 & $\begin{array}{l}5.5 \\
(5) \\
\end{array}$ & 0.22 & 0.5 & $\begin{array}{c}3 \\
(1.5) \\
\end{array}$ & 1.20 & 2 & 1.58 & A4 \\
\hline & 8-5-S6.5-2\#3-i-2.5-3-12.25 & $\begin{array}{l}A \\
B\end{array}$ & 4 & $\begin{array}{l}5.5 \\
(5) \\
\end{array}$ & 0.22 & 0.5 & $\begin{array}{c}3 \\
(1.5)\end{array}$ & 1.20 & 2 & 1.58 & A4 \\
\hline & 8-5-O4.5-2\#3-i-2.5-3-9.25 & $\begin{array}{l}A \\
B\end{array}$ & 4 & $\begin{array}{l}5.5 \\
(5) \\
\end{array}$ & 0.22 & 0.5 & $\begin{array}{c}3 \\
(1.5)\end{array}$ & 1.20 & 2 & 1.58 & A4 \\
\hline & 8-5-O4.5-2\#3-i-2.5-3-12.25 & $\begin{array}{l}\text { A } \\
B\end{array}$ & 4 & $\begin{array}{l}5.5 \\
(5) \\
\end{array}$ & 0.22 & 0.5 & $\begin{array}{c}3 \\
(1.5) \\
\end{array}$ & 1.20 & 2 & 1.58 & A4 \\
\hline & 8-5-S6.5-5\#3-i-2.5-3-8.25 & $\begin{array}{l}\text { A } \\
\text { B }\end{array}$ & 10 & $\begin{array}{c}3 \\
(1.5) \\
\end{array}$ & 0.66 & 0.5 & $\begin{array}{c}2.5 \\
(1.25) \\
\end{array}$ & 1.20 & 2 & 1.58 & A4 \\
\hline & 8-5-S6.5-5\#3-i-2.5-3-11.25 & $\begin{array}{l}\mathrm{A} \\
\mathrm{B}\end{array}$ & 10 & $\begin{array}{c}3 \\
(1.5) \\
\end{array}$ & 0.66 & 0.5 & $\begin{array}{c}3 \\
(1.5) \\
\end{array}$ & 1.20 & 2 & 1.58 & A4 \\
\hline & $8-5-04.5-5 \# 3-i-2.5-3-8.25$ & $\begin{array}{l}\mathrm{A} \\
\mathrm{B}\end{array}$ & 10 & $\begin{array}{c}3 \\
(1.5) \\
\end{array}$ & 0.66 & 0.5 & $\begin{array}{c}2.5 \\
(1.25)\end{array}$ & 1.20 & 2 & 1.58 & A4 \\
\hline & 8-5-O4.5-5\#3-i-2.5-3-11.25 & $\begin{array}{l}\text { A } \\
B\end{array}$ & 10 & $\begin{array}{c}3 \\
(1.5)\end{array}$ & 0.66 & 0.5 & $\begin{array}{c}3 \\
(1.5)\end{array}$ & 1.20 & 2 & 1.58 & A4 \\
\hline \multirow{5}{*}{ Group 7} & 8-5-T9.5-0-i-2.5-3-14.5 & $\begin{array}{l}\text { A } \\
\text { B }\end{array}$ & - & - & - & 0.375 & $\begin{array}{c}4 \\
(2) \\
\end{array}$ & 0.44 & 2 & 1.58 & A4 \\
\hline & 8-5-09.1-0-i-2.5-3-14.5 & $\begin{array}{l}\text { A } \\
B\end{array}$ & - & - & - & 0.375 & $\begin{array}{c}4 \\
(2) \\
\end{array}$ & 0.44 & 2 & 1.58 & A4 \\
\hline & 8-5-T9.5-5\#3-i-2.5-3-14.5 & $\begin{array}{l}\text { A } \\
B\end{array}$ & 10 & $\begin{array}{c}3 \\
(1.5) \\
\end{array}$ & 0.66 & 0.375 & $\begin{array}{c}4 \\
(2) \\
\end{array}$ & 0.44 & 2 & 1.58 & A6 \\
\hline & 8-5-09.1-5\#3-i-2.5-3-14.5 & $\begin{array}{l}\text { A } \\
B\end{array}$ & 10 & $\begin{array}{c}3 \\
(1.5) \\
\end{array}$ & 0.66 & 0.375 & $\begin{array}{c}4 \\
(2) \\
\end{array}$ & 0.28 & 2 & 1.58 & A6 \\
\hline & (3@5.5)8-5-T9.5-0-i-2.5-3-14.5 & $\begin{array}{l}\text { A } \\
\text { B } \\
\text { C }\end{array}$ & - & - & - & 0.375 & $\begin{array}{c}4 \\
(2)\end{array}$ & 0.44 & 3 & 2.37 & A5 \\
\hline
\end{tabular}

\footnotetext{
${ }^{*}$ Value in parenthesis is the spacing between the first hoop and the center of the headed bar
} 
Table A.2 Cont. Comprehensive test results and data for beam-column joint specimens

\begin{tabular}{|c|c|c|c|c|c|c|c|c|c|c|}
\hline & Specimen & Head & $\begin{array}{c}\text { Failure } \\
\text { Type }\end{array}$ & $\begin{array}{c}\text { Lead (Head) } \\
\text { Slip } \\
\text { in. }\end{array}$ & $\begin{array}{l}\boldsymbol{T}_{\max } \\
\text { kips }\end{array}$ & $\begin{array}{c}\boldsymbol{f}_{s u, \text { max }} \\
\text { ksi }\end{array}$ & $\begin{array}{l}\boldsymbol{T}_{\text {ind }} \\
\text { kips }\end{array}$ & $\begin{array}{l}\boldsymbol{T}_{\text {total }} \\
\text { kips }\end{array}$ & $\begin{array}{c}\boldsymbol{T} \\
\text { kips }\end{array}$ & $\begin{array}{l}f_{\text {su }} \\
\mathrm{ksi}\end{array}$ \\
\hline \multirow{3}{*}{ Group 5} & (3@4)8-12-F4.1-5\#3-i-2.5-3-10 & $\begin{array}{l}\text { A } \\
\text { B } \\
\text { C }\end{array}$ & $\mathrm{CB} / \mathrm{FP}$ & $\begin{array}{l}0.138 \\
0.240 \\
0.260\end{array}$ & $\begin{array}{l}64.0 \\
66.5 \\
66.7\end{array}$ & $\begin{array}{l}81.0 \\
84.2 \\
84.4\end{array}$ & $\begin{array}{l}64.0 \\
66.5 \\
66.6\end{array}$ & 197.1 & 65.7 & 83.2 \\
\hline & (3@5)8-12-F4.1-0-i-2.5-3-10 & $\begin{array}{l}\text { A } \\
\text { B } \\
\text { C }\end{array}$ & CB & $\begin{array}{c}0.079 \\
0.177 \\
0.249(0.081) \\
\end{array}$ & $\begin{array}{l}57.1 \\
55.3 \\
53.0 \\
\end{array}$ & $\begin{array}{l}72.3 \\
70.0 \\
67.1 \\
\end{array}$ & $\begin{array}{l}57.1 \\
55.3 \\
53.0 \\
\end{array}$ & 165.4 & 55.1 & 69.7 \\
\hline & (3@5)8-12-F4.1-5\#3-i-2.5-3-10 & $\begin{array}{l}\text { A } \\
\text { B } \\
\text { C }\end{array}$ & $\mathrm{CB} / \mathrm{FP}$ & $\begin{array}{l}0.164 \\
0.123 \\
0.122 \\
\end{array}$ & $\begin{array}{l}77.2 \\
65.4 \\
66.7 \\
\end{array}$ & $\begin{array}{l}97.7 \\
82.8 \\
84.4 \\
\end{array}$ & $\begin{array}{l}77.2 \\
65.4 \\
66.6 \\
\end{array}$ & 209.1 & 69.7 & 88.2 \\
\hline \multirow{12}{*}{ Group 6} & 8-5-S6.5-0-i-2.5-3-11.25 & $\begin{array}{l}\mathrm{A} \\
\mathrm{B}\end{array}$ & $\mathrm{SB} / \mathrm{FP}$ & $\begin{array}{c}0.161 \\
- \\
\end{array}$ & $\begin{array}{l}74.9 \\
76.2 \\
\end{array}$ & $\begin{array}{l}94.8 \\
96.5 \\
\end{array}$ & $\begin{array}{l}74.9 \\
76.2 \\
\end{array}$ & 151.1 & 75.6 & 95.6 \\
\hline & 8-5-S6.5-0-i-2.5-3-14.25 & $\begin{array}{l}\mathrm{A} \\
\mathrm{B}\end{array}$ & $\mathrm{SB} / \mathrm{FP}$ & $\begin{array}{c}(0.054) \\
-\end{array}$ & $\begin{array}{c}87.5 \\
103.4 \\
\end{array}$ & $\begin{array}{l}110.8 \\
130.9\end{array}$ & $\begin{array}{l}87.5 \\
88.0\end{array}$ & 175.4 & 87.7 & 111.0 \\
\hline & 8-5-O4.5-0-i-2.5-3-11.25 & $\begin{array}{l}\text { A } \\
\text { B }\end{array}$ & $\mathrm{SB} / \mathrm{FP}$ & $\begin{array}{l}0.037 \\
0.198\end{array}$ & $\begin{array}{l}67.6 \\
67.2 \\
\end{array}$ & $\begin{array}{l}85.6 \\
85.0\end{array}$ & $\begin{array}{l}67.6 \\
67.2\end{array}$ & 134.8 & 67.4 & 85.3 \\
\hline & 8-5-O4.5-0-i-2.5-3-14.25 & $\begin{array}{l}\text { A } \\
B\end{array}$ & $\mathrm{SB} / \mathrm{FP}$ & $\begin{array}{c}0.214(0.023) \\
0.113\end{array}$ & $\begin{array}{c}103.5 \\
85.8\end{array}$ & $\begin{array}{l}131.0 \\
108.6\end{array}$ & $\begin{array}{l}84.2 \\
85.8\end{array}$ & 170.0 & 85.0 & 107.6 \\
\hline & 8-5-S6.5-2\#3-i-2.5-3-9.25 & $\begin{array}{l}\text { A } \\
B\end{array}$ & CB & $\begin{array}{c}(0.012) \\
-\end{array}$ & $\begin{array}{l}62.6 \\
64.1\end{array}$ & $\begin{array}{l}79.2 \\
81.2\end{array}$ & $\begin{array}{l}62.6 \\
64.1\end{array}$ & 126.7 & 63.4 & 80.2 \\
\hline & 8-5-S6.5-2\#3-i-2.5-3-12.25 & $\begin{array}{l}\mathrm{A} \\
\mathrm{B}\end{array}$ & $\mathrm{SB} / \mathrm{FP}$ & $\begin{array}{l}0.340 \\
0.254 \\
\end{array}$ & $\begin{array}{l}84.6 \\
89.3 \\
\end{array}$ & $\begin{array}{l}107.1 \\
113.0 \\
\end{array}$ & $\begin{array}{l}84.6 \\
87.3\end{array}$ & 171.9 & 86.0 & 108.8 \\
\hline & 8-5-O4.5-2\#3-i-2.5-3-9.25 & $\begin{array}{l}\mathrm{A} \\
\mathrm{B}\end{array}$ & $\mathrm{SB} / \mathrm{FP}$ & $\begin{array}{l}0.309 \\
0.205 \\
\end{array}$ & $\begin{array}{l}67.6 \\
68.7 \\
\end{array}$ & $\begin{array}{l}85.6 \\
86.9 \\
\end{array}$ & $\begin{array}{l}67.1 \\
68.7 \\
\end{array}$ & 135.8 & 67.9 & 86.0 \\
\hline & 8-5-O4.5-2\#3-i-2.5-3-12.25 & $\begin{array}{l}\mathrm{A} \\
\mathrm{B}\end{array}$ & $\mathrm{SB} / \mathrm{FP}$ & $\begin{array}{l}0.305 \\
0.220\end{array}$ & $\begin{array}{l}82.8 \\
79.6 \\
\end{array}$ & $\begin{array}{l}104.8 \\
100.8 \\
\end{array}$ & $\begin{array}{l}77.4 \\
79.6 \\
\end{array}$ & 157.0 & 78.5 & 99.4 \\
\hline & 8-5-S6.5-5\#3-i-2.5-3-8.25 & $\begin{array}{l}\text { A } \\
B\end{array}$ & $\mathrm{CB} / \mathrm{FP}$ & $\begin{array}{l}0.363 \\
0.500\end{array}$ & $\begin{array}{l}61.9 \\
62.2\end{array}$ & $\begin{array}{l}78.4 \\
78.7\end{array}$ & $\begin{array}{l}61.9 \\
62.2\end{array}$ & 124.1 & 62.0 & 78.5 \\
\hline & 8-5-S6.5-5\#3-i-2.5-3-11.25 & $\begin{array}{l}\mathrm{A} \\
\mathrm{B}\end{array}$ & $\mathrm{SB} / \mathrm{FP}$ & $\begin{array}{c}- \\
0.046 \\
\end{array}$ & $\begin{array}{c}100.8 \\
84.7 \\
\end{array}$ & $\begin{array}{l}127.6 \\
107.2 \\
\end{array}$ & $\begin{array}{l}84.2 \\
84.7 \\
\end{array}$ & 169.0 & 84.5 & 106.9 \\
\hline & 8-5-O4.5-5\#3-i-2.5-3-8.25 & $\begin{array}{l}\mathrm{A} \\
\mathrm{B}\end{array}$ & $\mathrm{SB} / \mathrm{FP}$ & $\begin{array}{l}0.457 \\
0.383 \\
\end{array}$ & $\begin{array}{l}68.3 \\
68.5 \\
\end{array}$ & $\begin{array}{l}86.5 \\
86.7 \\
\end{array}$ & $\begin{array}{l}68.3 \\
68.5 \\
\end{array}$ & 136.8 & 68.4 & 86.6 \\
\hline & 8-5-O4.5-5\#3-i-2.5-3-11.25 & $\begin{array}{l}\mathrm{A} \\
\mathrm{B}\end{array}$ & $\mathrm{SB} / \mathrm{FP}$ & $\begin{array}{c}0.171 \\
- \\
\end{array}$ & $\begin{array}{l}85.0 \\
82.4 \\
\end{array}$ & $\begin{array}{l}107.6 \\
104.3 \\
\end{array}$ & $\begin{array}{l}82.1 \\
82.4 \\
\end{array}$ & 164.5 & 82.2 & 104.1 \\
\hline \multirow{5}{*}{ Group 7} & 8-5-T9.5-0-i-2.5-3-14.5 & $\begin{array}{l}\text { A } \\
B\end{array}$ & $\mathrm{SB} / \mathrm{FP}$ & $\begin{array}{l}0.130 \\
0.312 \\
\end{array}$ & $\begin{array}{c}91.5 \\
115.9 \\
\end{array}$ & $\begin{array}{l}115.8 \\
146.7 \\
\end{array}$ & $\begin{array}{l}91.5 \\
91.8 \\
\end{array}$ & 183.3 & 91.7 & 116.0 \\
\hline & 8-5-09.1-0-i-2.5-3-14.5 & $\begin{array}{l}A \\
B\end{array}$ & $\mathrm{SB} / \mathrm{FP}$ & $\begin{array}{l}0.060 \\
0.186\end{array}$ & $\begin{array}{l}94.6 \\
95.2 \\
\end{array}$ & $\begin{array}{l}119.7 \\
120.5 \\
\end{array}$ & $\begin{array}{l}94.6 \\
95.0\end{array}$ & 189.6 & 94.8 & 120.0 \\
\hline & 8-5-T9.5-5\#3-i-2.5-3-14.5 & $\begin{array}{l}\mathrm{A} \\
\mathrm{B}\end{array}$ & $\mathrm{SB} / \mathrm{FP}$ & $\begin{array}{l}- \\
-\end{array}$ & $\begin{array}{l}120.7^{\ddagger} \\
121.4\end{array}$ & $\begin{array}{l}152.8^{\ddagger} \\
153.7\end{array}$ & $\begin{array}{l}120.6 \\
121.4\end{array}$ & 242.0 & 121.0 & 153.2 \\
\hline & 8-5-09.1-5\#3-i-2.5-3-14.5 & $\begin{array}{l}\mathrm{A} \\
\mathrm{B}\end{array}$ & $\mathrm{Y}$ & $\begin{array}{c}0.050 \\
- \\
\end{array}$ & $\begin{array}{l}118.8 \\
119.7 \\
\end{array}$ & $\begin{array}{l}150.4 \\
151.5 \\
\end{array}$ & $\begin{array}{l}118.8 \\
119.7 \\
\end{array}$ & 238.5 & 119.3 & 150.9 \\
\hline & (3@5.5)8-5-T9.5-0-i-2.5-3-14.5 & $\begin{array}{l}\mathrm{A} \\
\mathrm{B} \\
\mathrm{C}\end{array}$ & CB & $\begin{array}{l}0.156 \\
0.138 \\
0.217\end{array}$ & $\begin{array}{l}68.7 \\
78.8 \\
72.6\end{array}$ & $\begin{array}{l}87.0 \\
99.7 \\
91.9\end{array}$ & $\begin{array}{l}68.7 \\
78.8 \\
72.6\end{array}$ & 220.2 & 73.4 & 92.9 \\
\hline
\end{tabular}

\footnotetext{
${ }^{\ddagger}$ No anchorage failure on the bar
} 
Table A.2 Cont. Comprehensive test results and data for beam-column joint specimens

\begin{tabular}{|c|c|c|c|c|c|c|c|c|c|c|}
\hline & Specimen & Head & $\begin{array}{l}\boldsymbol{c}_{\boldsymbol{o}} \\
\text { in. }\end{array}$ & $A_{b r g}$ & $\begin{array}{l}\ell \text { eh } \\
\text { in. }\end{array}$ & $\begin{array}{c}\ell \text { eh, avg } \\
\text { in. }\end{array}$ & $\begin{array}{l}\boldsymbol{f}_{\mathrm{cm}} \\
\mathrm{psi}\end{array}$ & $\begin{array}{l}\text { Age } \\
\text { days }\end{array}$ & $\begin{array}{l}\boldsymbol{d}_{\boldsymbol{b}} \\
\text { in. }\end{array}$ & $\begin{array}{c}\boldsymbol{A}_{\boldsymbol{b}} \\
\text { in. }^{2}\end{array}$ \\
\hline \multirow{7}{*}{ Group 7} & (3@5.5)8-5-09.1-0-i-2.5-3-14.5 & $\begin{array}{l}\text { A } \\
B \\
C\end{array}$ & 1.3 & $9.1 A_{b}$ & $\begin{array}{l}14.31 \\
14.50 \\
14.25 \\
\end{array}$ & 14.35 & 4960 & 9 & 1 & 0.79 \\
\hline & (3@5.5)8-5-T9.5-5\#3-i-2.5-3-14.5 & $\begin{array}{l}\text { A } \\
B \\
C\end{array}$ & 1.4 & $9.5 A_{b}$ & $\begin{array}{l}14.50 \\
14.38 \\
14.38\end{array}$ & 14.42 & 5370 & 10 & 1 & 0.79 \\
\hline & (3@5.5)8-5-09.1-5\#3-i-2.5-3-14.5 & $\begin{array}{l}\text { A } \\
B \\
C\end{array}$ & 1.3 & $9.1 A_{b}$ & $\begin{array}{l}14.06 \\
14.44 \\
14.31\end{array}$ & 14.27 & 5420 & 13 & 1 & 0.79 \\
\hline & (4@3.7)8-5-T9.5-0-i-2.5-3-14.5 & $\begin{array}{l}\text { A } \\
\text { B } \\
\text { C } \\
\text { D }\end{array}$ & 1.4 & $9.5 A_{b}$ & $\begin{array}{l}14.25 \\
14.38 \\
14.25 \\
14.19\end{array}$ & 14.30 & 5570 & 14 & 1 & 0.79 \\
\hline & (4@3.7)8-5-09.1-0-i-2.5-3-14.5 & $\begin{array}{l}\text { A } \\
\text { B } \\
\text { C } \\
\text { D }\end{array}$ & 1.3 & $9.1 A_{b}$ & $\begin{array}{l}14.06 \\
14.06 \\
14.06 \\
14.06\end{array}$ & 14.06 & 5570 & 14 & 1 & 0.79 \\
\hline & (4@3.7)8-5-T9.5-5\#3-i-2.5-3-14.5 & $\begin{array}{l}\text { A } \\
B \\
C \\
D\end{array}$ & 1.4 & $9.5 A_{b}$ & $\begin{array}{l}14.44 \\
14.38 \\
14.63 \\
14.50\end{array}$ & 14.50 & 5570 & 14 & 1 & 0.79 \\
\hline & (4@3.7)8-5-09.1-5\#3-i-2.5-3-14.5 & $\begin{array}{l}\text { A } \\
\text { B } \\
\text { C } \\
\text { D }\end{array}$ & 1.3 & $9.1 A_{b}$ & $\begin{array}{l}14.44 \\
14.44 \\
14.63 \\
14.44\end{array}$ & 14.50 & 5570 & 14 & 1 & 0.79 \\
\hline \multirow{9}{*}{ Group 8} & 8-15-T4.0-0-i-2.5-4.5-9.5 & $\begin{array}{l}\text { A } \\
B\end{array}$ & 1.9 & $4.0 A_{b}$ & $\begin{array}{l}9.50 \\
9.50\end{array}$ & 9.50 & 16030 & 88 & 1 & 0.79 \\
\hline & 8-15-S9.5-0-i-2.5-3-9.5 & $\begin{array}{l}\mathrm{A} \\
\mathrm{B}\end{array}$ & 1.4 & $9.5 A_{b}$ & $\begin{array}{l}9.50 \\
9.50 \\
\end{array}$ & 9.50 & 16030 & 88 & 1 & 0.79 \\
\hline & 8-15-S14.9-0-i-2.5-3-9.5 & $\begin{array}{l}A \\
B\end{array}$ & 1.0 & $14.9 A_{b}$ & $\begin{array}{l}9.63 \\
9.75 \\
\end{array}$ & 9.69 & 16030 & 88 & 1 & 0.79 \\
\hline & 8-15-T4.0-2\#3-i-2.5-4.5-7 & $\begin{array}{l}A \\
B\end{array}$ & 1.9 & $4.0 A_{b}$ & $\begin{array}{l}7.13 \\
7.00 \\
\end{array}$ & 7.06 & 16030 & 87 & 1 & 0.79 \\
\hline & 8-15-S9.5-2\#3-i-2.5-3-7 & $\begin{array}{l}\text { A } \\
B\end{array}$ & 1.4 & $9.5 A_{b}$ & $\begin{array}{l}7.13 \\
7.00\end{array}$ & 7.06 & 16030 & 87 & 1 & 0.79 \\
\hline & 8-15-S14.9-2\#3-i-2.5-3-7 & $\begin{array}{l}\text { A } \\
B\end{array}$ & 1.0 & $14.9 A_{b}$ & $\begin{array}{l}7.00 \\
7.00\end{array}$ & 7.00 & 16030 & 87 & 1 & 0.79 \\
\hline & 8-15-T4.0-5\#3-i-2.5-4.5-5.5 & $\begin{array}{l}\text { A } \\
B\end{array}$ & 1.9 & $4.0 A_{b}$ & $\begin{array}{l}5.50 \\
5.50 \\
\end{array}$ & 5.50 & 16030 & 88 & 1 & 0.79 \\
\hline & 8-15-S9.5-5\#3-i-2.5-3-5.5 & $\begin{array}{l}\mathrm{A} \\
\mathrm{B}\end{array}$ & 1.4 & $9.5 A_{b}$ & $\begin{array}{l}5.75 \\
5.50 \\
\end{array}$ & 5.63 & 16030 & 88 & 1 & 0.79 \\
\hline & 8-15-S14.9-5\#3-i-2.5-3-5.5 & $\begin{array}{l}\text { A } \\
B\end{array}$ & 1.0 & $14.9 A_{b}$ & $\begin{array}{l}5.50 \\
5.50\end{array}$ & 5.50 & 16030 & 88 & 1 & 0.79 \\
\hline
\end{tabular}


Table A.2 Cont. Comprehensive test results and data for beam-column joint specimens

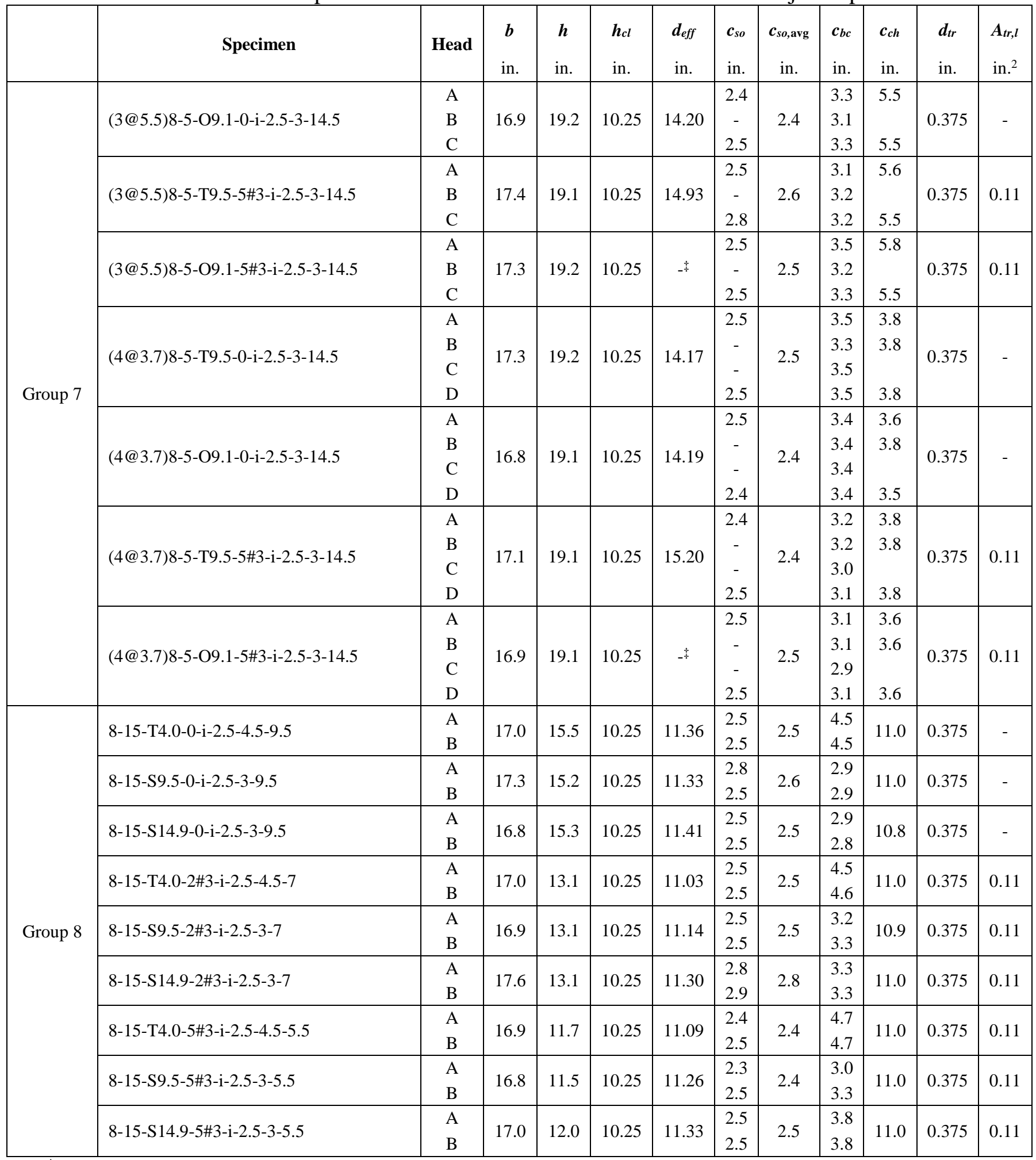

\footnotetext{
${ }^{\ddagger} d_{\text {eff }}$ was not calculated for specimen with bar yielding
} 
Table A.2 Cont. Comprehensive test results and data for beam-column joint specimens

\begin{tabular}{|c|c|c|c|c|c|c|c|c|c|c|c|}
\hline & Specimen & Head & $N$ & $\begin{array}{l}\boldsymbol{S}_{t r}{ }^{*} \\
\text { in. }\end{array}$ & $\begin{array}{l}\boldsymbol{A}_{\boldsymbol{t t}} \\
\text { in. }^{2}\end{array}$ & $\begin{array}{l}\boldsymbol{d}_{\text {tro }} \\
\text { in. }\end{array}$ & $\begin{array}{c}\text { Stro }^{*} \\
\text { in. }\end{array}$ & $\begin{array}{l}\boldsymbol{A}_{\boldsymbol{a} \boldsymbol{b}} \\
\text { in. }^{2}\end{array}$ & $n$ & $\begin{array}{l}\boldsymbol{A}_{\boldsymbol{h s}} \\
\text { in. }^{2}\end{array}$ & $\begin{array}{l}\text { Long. } \\
\text { Reinf. } \\
\text { Layout }\end{array}$ \\
\hline \multirow{7}{*}{ Group 7} & (3@5.5)8-5-09.1-0-i-2.5-3-14.5 & $\begin{array}{l}\text { A } \\
B \\
C\end{array}$ & - & - & - & 0.375 & $\begin{array}{c}4 \\
(2)\end{array}$ & 0.44 & 3 & 2.37 & A5 \\
\hline & (3@5.5)8-5-T9.5-5\#3-i-2.5-3-14.5 & $\begin{array}{l}\mathrm{A} \\
\mathrm{B} \\
\mathrm{C}\end{array}$ & 10 & $\begin{array}{c}3 \\
(1.5)\end{array}$ & 0.66 & 0.375 & $\begin{array}{c}4 \\
(2)\end{array}$ & 0.44 & 3 & 2.37 & A5 \\
\hline & (3@5.5)8-5-09.1-5\#3-i-2.5-3-14.5 & $\begin{array}{l}\text { A } \\
B \\
C\end{array}$ & 10 & $\begin{array}{c}3 \\
(1.5)\end{array}$ & 0.66 & 0.375 & $\begin{array}{c}4 \\
(2)\end{array}$ & 0.19 & 3 & 2.37 & A5 \\
\hline & (4@3.7)8-5-T9.5-0-i-2.5-3-14.5 & $\begin{array}{l}\text { A } \\
\text { B } \\
\text { C } \\
\text { D }\end{array}$ & - & - & - & 0.375 & $\begin{array}{c}4 \\
(2)\end{array}$ & 0.44 & 4 & 3.16 & A5 \\
\hline & (4@3.7)8-5-09.1-0-i-2.5-3-14.5 & $\begin{array}{l}\text { A } \\
\text { B } \\
\text { C } \\
\text { D }\end{array}$ & - & - & - & 0.375 & $\begin{array}{c}4 \\
(2)\end{array}$ & 0.44 & 4 & 3.16 & A5 \\
\hline & (4@3.7)8-5-T9.5-5\#3-i-2.5-3-14.5 & $\begin{array}{l}\text { A } \\
\text { B } \\
\text { C } \\
\text { D }\end{array}$ & 10 & $\begin{array}{c}3 \\
(1.5)\end{array}$ & 0.66 & 0.375 & $\begin{array}{c}3 \\
(1.5)\end{array}$ & 0.66 & 4 & 3.16 & A5 \\
\hline & (4@3.7)8-5-09.1-5\#3-i-2.5-3-14.5 & $\begin{array}{l}\mathrm{A} \\
\mathrm{B} \\
\mathrm{C} \\
\mathrm{D}\end{array}$ & 10 & $\begin{array}{c}3 \\
(1.5)\end{array}$ & 0.66 & 0.375 & $\begin{array}{c}3 \\
(1.5)\end{array}$ & 0.21 & 4 & 3.16 & A5 \\
\hline \multirow{9}{*}{ Group 8} & 8-15-T4.0-0-i-2.5-4.5-9.5 & $\begin{array}{l}A \\
B\end{array}$ & - & - & - & 0.5 & $\begin{array}{c}4 \\
(2) \\
\end{array}$ & 0.80 & 2 & 1.58 & A6 \\
\hline & 8-15-S9.5-0-i-2.5-3-9.5 & $\begin{array}{l}\mathrm{A} \\
\mathrm{B}\end{array}$ & - & - & - & 0.5 & $\begin{array}{c}4 \\
(2) \\
\end{array}$ & 0.80 & 2 & 1.58 & A6 \\
\hline & 8-15-S14.9-0-i-2.5-3-9.5 & $\begin{array}{l}A \\
B\end{array}$ & - & - & - & 0.5 & $\begin{array}{c}4 \\
(2) \\
\end{array}$ & 0.80 & 2 & 1.58 & A6 \\
\hline & 8-15-T4.0-2\#3-i-2.5-4.5-7 & $\begin{array}{l}\text { A } \\
B\end{array}$ & 4 & $\begin{array}{l}5.5 \\
(5) \\
\end{array}$ & 0.22 & 0.5 & $\begin{array}{c}4 \\
(2) \\
\end{array}$ & 0.80 & 2 & 1.58 & A6 \\
\hline & 8-15-S9.5-2\#3-i-2.5-3-7 & $\begin{array}{l}\text { A } \\
B\end{array}$ & 4 & $\begin{array}{l}5.5 \\
(5)\end{array}$ & 0.22 & 0.5 & $\begin{array}{c}4 \\
(2)\end{array}$ & 0.80 & 2 & 1.58 & A6 \\
\hline & 8-15-S14.9-2\#3-i-2.5-3-7 & $\begin{array}{l}A \\
B\end{array}$ & 4 & $\begin{array}{l}5.5 \\
(5)\end{array}$ & 0.22 & 0.5 & $\begin{array}{c}44 \\
(2)\end{array}$ & 0.80 & 2 & 1.58 & A6 \\
\hline & 8-15-T4.0-5\#3-i-2.5-4.5-5.5 & $\begin{array}{l}\text { A } \\
\text { B }\end{array}$ & 10 & $\begin{array}{c}3 \\
(1.5) \\
\end{array}$ & 0.66 & 0.5 & $\begin{array}{c}4 \\
(2) \\
\end{array}$ & 0.80 & 2 & 1.58 & A7 \\
\hline & 8-15-S9.5-5\#3-i-2.5-3-5.5 & $\begin{array}{l}\text { A } \\
B\end{array}$ & 10 & $\begin{array}{c}3 \\
(1.5) \\
\end{array}$ & 0.66 & 0.5 & $\begin{array}{c}4 \\
(2) \\
\end{array}$ & 0.80 & 2 & 1.58 & A7 \\
\hline & 8-15-S14.9-5\#3-i-2.5-3-5.5 & $\begin{array}{l}A \\
B\end{array}$ & 10 & $\begin{array}{c}3 \\
(1.5)\end{array}$ & 0.66 & 0.5 & $\begin{array}{c}4 \\
(2)\end{array}$ & 0.80 & 2 & 1.58 & A7 \\
\hline
\end{tabular}

${ }^{*}$ Value in parenthesis is the spacing between the first hoop and the center of the headed bar 
Table A.2 Cont. Comprehensive test results and data for beam-column joint specimens

\begin{tabular}{|c|c|c|c|c|c|c|c|c|c|c|}
\hline & Specimen & Head & $\begin{array}{c}\text { Failure } \\
\text { Type }\end{array}$ & $\begin{array}{c}\text { Lead (Head) } \\
\text { Slip } \\
\text { in. }\end{array}$ & $\begin{array}{l}\boldsymbol{T}_{\max } \\
\text { kips }\end{array}$ & $\begin{array}{c}\boldsymbol{f}_{s u, \text { max }} \\
\mathrm{ksi}\end{array}$ & $\begin{array}{l}\boldsymbol{T}_{\text {ind }} \\
\text { kips }\end{array}$ & $\begin{array}{c}\boldsymbol{T}_{\text {total }} \\
\text { kips }\end{array}$ & $\begin{array}{c}\boldsymbol{T} \\
\text { kips }\end{array}$ & $\begin{array}{l}\boldsymbol{f}_{\text {su }} \\
\mathrm{ksi}\end{array}$ \\
\hline \multirow{25}{*}{ Group 7} & \multirow{3}{*}{ (3@5.5)8-5-O9.1-0-i-2.5-3-14.5 } & A & \multirow{3}{*}{ CB } & $0.081(0.043)$ & 91.0 & 115.2 & 91.0 & \multirow{3}{*}{227.1} & \multirow{3}{*}{75.7} & \multirow{3}{*}{95.8} \\
\hline & & B & & 0.085 & 76.2 & 96.5 & 76.2 & & & \\
\hline & & $\mathrm{C}$ & & 0.055 & 59.9 & 75.8 & 59.9 & & & \\
\hline & \multirow{3}{*}{ (3@5.5)8-5-T9.5-5\#3-i-2.5-3-14.5 } & A & \multirow{3}{*}{ CB } & 0.121 & 91.5 & 115.8 & 91.5 & \multirow{3}{*}{283.8} & \multirow{3}{*}{94.6} & \multirow{3}{*}{119.7} \\
\hline & & B & & 0.086 & 91.5 & 115.8 & 91.5 & & & \\
\hline & & $\mathrm{C}$ & & 0.223 & 100.8 & 127.6 & 100.8 & & & \\
\hline & \multirow{3}{*}{ (3@5.5)8-5-O9.1-5\#3-i-2.5-3-14.5 } & A & \multirow{3}{*}{ Y } & - & 115.4 & 146.1 & 102.9 & \multirow{3}{*}{306.6} & \multirow{3}{*}{102.2} & \multirow{3}{*}{129.4} \\
\hline & & B & & - & 104.4 & 132.2 & 100.4 & & & \\
\hline & & $\mathrm{C}$ & & - & 103.3 & 130.8 & 103.3 & & & \\
\hline & \multirow{4}{*}{ (4@3.7)8-5-T9.5-0-i-2.5-3-14.5 } & A & \multirow{4}{*}{ CB } & 0.159 & 89.7 & 113.5 & 89.7 & \multirow{4}{*}{243.3} & \multirow{4}{*}{60.8} & \multirow{4}{*}{77.0} \\
\hline & & B & & 0.236 & 46.9 & 59.4 & 46.9 & & & \\
\hline & & $\mathrm{C}$ & & - & 57.6 & 72.9 & 57.6 & & & \\
\hline & & $\mathrm{D}$ & & 0.168 & 49.1 & 62.2 & 49.1 & & & \\
\hline & \multirow{4}{*}{ (4@3.7)8-5-09.1-0-i-2.5-3-14.5 } & $\mathrm{A}$ & & 0.088 & 67.9 & 85.9 & 67.9 & & & \\
\hline & & B & $\mathrm{CB}$ & - & 69.7 & 88.2 & 69.7 & 2449 & 612 & 775 \\
\hline & & $\mathrm{C}$ & & - & 56.6 & 71.6 & 56.6 & 244.9 & 01.2 & 11.3 \\
\hline & & $\mathrm{D}$ & & $0.114(0.085)$ & 50.8 & 64.3 & 50.8 & & & \\
\hline & & A & & 0.320 & $--^{\dagger}$ & $--^{\dagger}$ & - & & & \\
\hline & 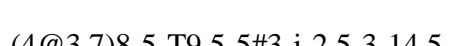 & B & $C \mathrm{P}_{2}$ & - & 82.2 & 104.1 & 82.2 & $\dagger$ & $76 a^{\dagger}$ & 072 \\
\hline & (4) & $\mathrm{C}$ & C & - & 74.6 & 94.4 & 74.6 & - & 10.5 & (3) \\
\hline & & D & & 0.161 & 73.8 & 93.4 & 73.8 & & & \\
\hline & & A & & 0.087 & $-^{\dagger}$ & $-^{\dagger}$ & - & & & \\
\hline & (1ค3 7)8 5 & B & $\mathrm{Y}$ & 0.016 & 97.1 & 122.9 & 96.8 & $t$ & $001 t$ & \\
\hline & (4@3./)8-5-09.1-5\#3-1-2.5-3-14.5 & $\mathrm{C}$ & $\mathrm{Y}$ & - & 88.9 & 112.5 & 88.9 & -1 & $89.1^{\prime}$ & 112.8 \\
\hline & & $\mathrm{D}$ & & 0.033 & 81.4 & 103.0 & 81.4 & & & \\
\hline & 8-15-T4 $0-0-\mathrm{i}_{-2} 5-45-95$ & A & $C \mathrm{~B}$ & - & 83.2 & 105.3 & 83.2 & 1666 & 833 & 105.4 \\
\hline & $0-15-14.0-0-1-2.5-4.5-9.5$ & B & CB & 0.237 & 83.4 & 105.6 & 83.4 & 100.0 & 03.3 & 105.4 \\
\hline & 8-15-S9 5-0-i-2 5-3-9 5 & A & $\mathrm{CB}$ & - & 83.5 & 105.7 & 83.5 & 1633 & 817 & 1034 \\
\hline & 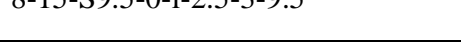 & B & C & - & 79.9 & 101.1 & 79.9 & 100.0 & 01.1 & 100.4 \\
\hline & & A & & - & 88.2 & 111.6 & 88.2 & & & \\
\hline & 8-15-S14.9-0-i-2.5-3-9.5 & B & CB & - & 86.1 & 109.0 & 86.1 & 174.2 & 87.1 & 110.3 \\
\hline & $8-15-T 10-2+3-25-45-7$ & A & $C \mathrm{R}_{2}$ & - & 59.1 & 74.8 & 59.1 & & & \\
\hline & $8-15-14.0-2 \# 3-1-2.5-4.5-1$ & $\mathrm{~B}$ & CB & - & 58.9 & 74.6 & 58.9 & 118.0 & 59.0 & 74.7 \\
\hline Croun 8 & 8 -15_s9 5-2\#3_i_-2 5-3-7 & A & $C B$ & - & 66.4 & 84.1 & 66.4 & 1212 & 671 & 840 \\
\hline Gioup o & $0-12-29.7-5+3-1-5.3-3-1$ & B & $C D$ & - & 67.9 & 85.9 & 67.9 & 134.3 & $6 / .1$ & 84.9 \\
\hline & 8-15-S14 9-2\#3-i-2 5-3-7 & A & $C \mathrm{~B}_{\mathrm{C}} \mathrm{C}_{\mathrm{C}}$ & - & 79.7 & 100.9 & 79.7 & 1587 & 793 & 1004 \\
\hline & 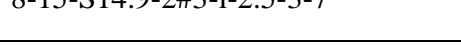 & B & CD & - & 78.9 & 99.9 & 78.9 & $100 . r^{\prime}$ & 17.0 & 100.4 \\
\hline & & A & $C P$ & - & 64.0 & 81.0 & 64.0 & $\mathrm{C}^{-1}$ & $6 ? 2$ & 1 \\
\hline & $8-15-14.0-5 \# 3-1-2.5-4.5-5.5$ & B & CB & - & 62.6 & 79.2 & 62.6 & $1 \angle 6.6$ & 03.3 & 80.1 \\
\hline & $8-15$ So $5-5 H 2-i-2-55$ & A & $C \mathrm{R}$ & - & 76.6 & 97.0 & 76.6 & & & \\
\hline & 8-15-S9.J-5\#3-1-2.J-3-5.J & B & CB & - & 75.0 & 94.9 & 75.0 & 151.6 & 75.8 & 95.9 \\
\hline & 915 c1405\#2 i 25255 & A & $C \mathrm{D}$ & - & 80.7 & 102.2 & 80.7 & 67 & 14 & 20 \\
\hline & 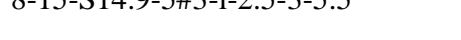 & B & CD & - & 82.0 & 103.8 & 82.0 & $102 . r^{\prime}$ & 01.4 & 103.0 \\
\hline
\end{tabular}

\footnotetext{
${ }^{\dagger}$ Load on headed bar A was not recorded due to a malfunction of load cell; $T$ taken as the average load of the other three bars.
} 
Table A.2 Cont. Comprehensive test results and data for beam-column joint specimens

\begin{tabular}{|c|c|c|c|c|c|c|c|c|c|c|}
\hline & Specimen & Head & $\begin{array}{l}\boldsymbol{c}_{o} \\
\text { in. }\end{array}$ & $A_{b r g}$ & $\begin{array}{l}\ell \text { eh } \\
\text { in. }\end{array}$ & $\begin{array}{c}\ell \text { eh, avg } \\
\text { in. }\end{array}$ & $\begin{array}{l}\boldsymbol{f}_{\boldsymbol{c m}} \\
\mathrm{psi}\end{array}$ & $\begin{array}{l}\text { Age } \\
\text { days }\end{array}$ & $\begin{array}{l}\boldsymbol{d}_{\boldsymbol{b}} \\
\text { in. }\end{array}$ & $\begin{array}{c}\boldsymbol{A}_{\boldsymbol{b}} \\
\text { in. }^{2}\end{array}$ \\
\hline \multirow{15}{*}{ Group 9} & 8-8-T9.5-0-i-2.5-3-9.5 & $\begin{array}{l}A \\
B\end{array}$ & 1.4 & $9.5 A_{b}$ & $\begin{array}{l}9.50 \\
9.25\end{array}$ & 9.38 & 9040 & 12 & 1 & 0.79 \\
\hline & 8-8-T9.5-2\#3-i-2.5-3-9.5 & $\begin{array}{l}A \\
B\end{array}$ & 1.4 & $9.5 A_{b}$ & $\begin{array}{l}9.00 \\
9.38\end{array}$ & 9.19 & 9040 & 12 & 1 & 0.79 \\
\hline & (3@4)8-8-T9.5-0-i-2.5-3-9.5 & $\begin{array}{l}\text { A } \\
B \\
C\end{array}$ & 1.4 & $9.5 A_{b}$ & $\begin{array}{l}9.00 \\
9.50 \\
9.25\end{array}$ & 9.25 & 9040 & 12 & 1 & 0.79 \\
\hline & (3@4)8-8-T9.5-2\#3-i-2.5-3-9.5 & $\begin{array}{l}\text { A } \\
B \\
C\end{array}$ & 1.4 & $9.5 A_{b}$ & $\begin{array}{l}9.75 \\
9.50 \\
9.50\end{array}$ & 9.58 & 9040 & 12 & 1 & 0.79 \\
\hline & (3@5)8-8-T9.5-0-i-2.5-3-9.5 & $\begin{array}{l}\text { A } \\
\text { B } \\
\text { C }\end{array}$ & 1.4 & $9.5 A_{b}$ & $\begin{array}{l}9.50 \\
9.75 \\
9.25\end{array}$ & 9.50 & 9940 & 11 & 1 & 0.79 \\
\hline & (3@5)8-8-T9.5-2\#3-i-2.5-3-9.5 & $\begin{array}{l}\text { A } \\
B \\
C\end{array}$ & 1.4 & $9.5 A_{b}$ & $\begin{array}{l}9.50 \\
9.50 \\
9.25\end{array}$ & 9.42 & 9940 & 11 & 1 & 0.79 \\
\hline & (3@7)8-8-T9.5-0-i-2.5-3-9.5 & $\begin{array}{l}A \\
B \\
C\end{array}$ & 1.4 & $9.5 A_{b}$ & $\begin{array}{l}9.50 \\
9.63 \\
9.38 \\
\end{array}$ & 9.50 & 10180 & 10 & 1 & 0.79 \\
\hline & (3@7)8-8-T9.5-2\#3-i-2.5-3-9.5 & $\begin{array}{l}\text { A } \\
B \\
C\end{array}$ & 1.4 & $9.5 A_{b}$ & $\begin{array}{l}9.50 \\
9.75 \\
9.50 \\
\end{array}$ & 9.58 & 10180 & 10 & 1 & 0.79 \\
\hline & 8-8-T9.5-0-i-2.5-3-14.5 & $\begin{array}{l}A \\
B\end{array}$ & 1.4 & $9.5 A_{b}$ & $\begin{array}{l}14.50 \\
14.25\end{array}$ & 14.38 & 10180 & 10 & 1 & 0.79 \\
\hline & (3@4)8-8-T9.5-0-i-2.5-3-14.5 & $\begin{array}{l}\text { A } \\
B \\
C \\
\end{array}$ & 1.4 & $9.5 A_{b}$ & $\begin{array}{l}14.25 \\
14.75 \\
14.75 \\
\end{array}$ & 14.58 & 9040 & 12 & 1 & 0.79 \\
\hline & (3@4)8-8-T9.5-2\#3-i-2.5-3-14.5 & $\begin{array}{l}\text { A } \\
B \\
C\end{array}$ & 1.4 & $9.5 A_{b}$ & $\begin{array}{l}14.50 \\
14.50 \\
14.25 \\
\end{array}$ & 14.42 & 9040 & 12 & 1 & 0.79 \\
\hline & (3@5)8-8-T9.5-0-i-2.5-3-14.5 & $\begin{array}{l}\text { A } \\
\text { B } \\
\text { C }\end{array}$ & 1.4 & $9.5 A_{b}$ & $\begin{array}{l}14.75 \\
14.50 \\
14.50\end{array}$ & 14.58 & 9940 & 11 & 1 & 0.79 \\
\hline & (3@5)8-8-T9.5-2\#3-i-2.5-3-14.5 & $\begin{array}{l}\text { A } \\
\text { B } \\
\text { C }\end{array}$ & 1.4 & $9.5 A_{b}$ & $\begin{array}{l}14.00 \\
14.25 \\
14.00\end{array}$ & 14.08 & 9940 & 11 & 1 & 0.79 \\
\hline & (3@7)8-8-T9.5-0-i-2.5-3-14.5 & $\begin{array}{l}\mathrm{A} \\
\mathrm{B} \\
\mathrm{C}\end{array}$ & 1.4 & $9.5 A_{b}$ & $\begin{array}{l}14.44 \\
14.56 \\
14.63 \\
\end{array}$ & 14.54 & 10180 & 10 & 1 & 0.79 \\
\hline & (3@7)8-8-T9.5-2\#3-i-2.5-3-14.5 & $\begin{array}{l}\text { A } \\
B \\
C\end{array}$ & 1.4 & $9.5 A_{b}$ & $\begin{array}{l}14.50 \\
14.63 \\
14.50\end{array}$ & 14.54 & 10180 & 10 & 1 & 0.79 \\
\hline
\end{tabular}


Table A.2 Cont. Comprehensive test results and data for beam-column joint specimens

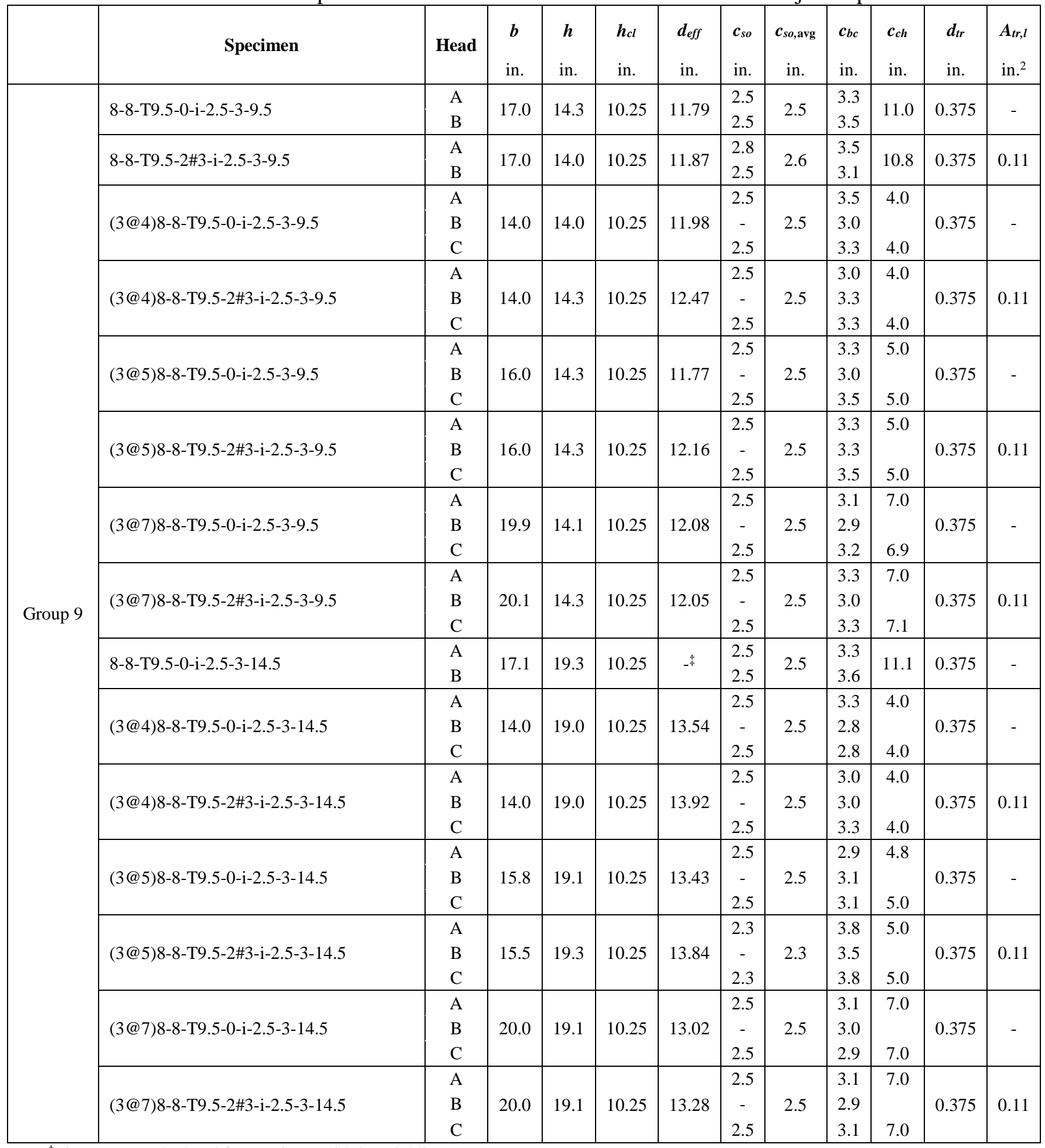

\footnotetext{
${ }^{\ddagger} d_{\text {eff }}$ was not calculated for specimen with bar yielding
} 
Table A.2 Cont. Comprehensive test results and data for beam-column joint specimens

\begin{tabular}{|c|c|c|c|c|c|c|c|c|c|c|c|}
\hline & Specimen & Head & $N$ & $\begin{array}{l}s_{t r}{ }^{*} \\
\text { in. }\end{array}$ & $\begin{array}{l}\boldsymbol{A}_{\boldsymbol{t t}} \\
\text { in. }^{2}\end{array}$ & $\begin{array}{l}\boldsymbol{d}_{\text {tro }} \\
\text { in. }\end{array}$ & $\begin{array}{c}\boldsymbol{S}_{\text {tro }} * \\
\text { in. }\end{array}$ & $\begin{array}{l}\boldsymbol{A}_{a b} \\
\text { in. }^{2}\end{array}$ & $n$ & $\begin{array}{l}A_{h s} \\
\text { in. }^{2}\end{array}$ & $\begin{array}{l}\text { Long. } \\
\text { Reinf. } \\
\text { Layout }\end{array}$ \\
\hline \multirow{15}{*}{ Group 9} & 8-8-T9.5-0-i-2.5-3-9.5 & $\begin{array}{l}\text { A } \\
B\end{array}$ & - & - & - & 0.375 & $\begin{array}{c}3 \\
(1.5)\end{array}$ & 0.66 & 2 & 1.58 & A6 \\
\hline & 8-8-T9.5-2\#3-i-2.5-3-9.5 & $\begin{array}{l}\mathrm{A} \\
\mathrm{B}\end{array}$ & 4 & $\begin{array}{c}6 \\
(4.5) \\
\end{array}$ & 0.22 & 0.375 & $\begin{array}{c}3 \\
(1.5) \\
\end{array}$ & 0.66 & 2 & 1.58 & A6 \\
\hline & (3@4)8-8-T9.5-0-i-2.5-3-9.5 & $\begin{array}{l}\text { A } \\
B \\
C\end{array}$ & - & - & - & 0.375 & $\begin{array}{c}3 \\
(1.5)\end{array}$ & 0.66 & 3 & 2.37 & A5 \\
\hline & (3@4)8-8-T9.5-2\#3-i-2.5-3-9.5 & $\begin{array}{l}A \\
B \\
C\end{array}$ & 4 & $\begin{array}{c}6 \\
(4.5)\end{array}$ & 0.22 & 0.375 & $\begin{array}{c}3 \\
(1.5)\end{array}$ & 0.66 & 3 & 2.37 & A5 \\
\hline & (3@5)8-8-T9.5-0-i-2.5-3-9.5 & $\begin{array}{l}A \\
B \\
C\end{array}$ & - & - & - & 0.375 & $\begin{array}{c}3 \\
(1.5)\end{array}$ & 0.66 & 3 & 2.37 & A5 \\
\hline & (3@5)8-8-T9.5-2\#3-i-2.5-3-9.5 & $\begin{array}{l}\text { A } \\
B \\
C\end{array}$ & 4 & $\begin{array}{c}6 \\
(4.5)\end{array}$ & 0.22 & 0.375 & $\begin{array}{c}3 \\
(1.5)\end{array}$ & 0.66 & 3 & 2.37 & A5 \\
\hline & (3@7)8-8-T9.5-0-i-2.5-3-9.5 & $\begin{array}{l}A \\
B \\
C\end{array}$ & - & - & - & 0.5 & $\begin{array}{c}4.5 \\
(2.25)\end{array}$ & 0.80 & 3 & 2.37 & A7 \\
\hline & (3@7)8-8-T9.5-2\#3-i-2.5-3-9.5 & $\begin{array}{l}\text { A } \\
\text { B } \\
\text { C } \\
\end{array}$ & 4 & $\begin{array}{c}6 \\
(4.5)\end{array}$ & 0.22 & 0.5 & $\begin{array}{c}4.5 \\
(2.25)\end{array}$ & 0.80 & 3 & 2.37 & A7 \\
\hline & 8-8-T9.5-0-i-2.5-3-14.5 & $\begin{array}{l}\text { A } \\
B\end{array}$ & - & - & - & 0.375 & $\begin{array}{c}4 \\
(2) \\
\end{array}$ & 0.28 & 2 & 1.58 & A6 \\
\hline & (3@4)8-8-T9.5-0-i-2.5-3-14.5 & $\begin{array}{l}\text { A } \\
B \\
C\end{array}$ & - & - & - & 0.375 & $\begin{array}{c}4 \\
(2)\end{array}$ & 0.44 & 3 & 2.37 & A5 \\
\hline & (3@4)8-8-T9.5-2\#3-i-2.5-3-14.5 & $\begin{array}{l}\text { A } \\
B \\
C \\
\end{array}$ & 4 & $\begin{array}{c}6 \\
(4.5)\end{array}$ & 0.22 & 0.375 & $\begin{array}{c}4 \\
(2)\end{array}$ & 0.44 & 3 & 2.37 & A5 \\
\hline & (3@5)8-8-T9.5-0-i-2.5-3-14.5 & $\begin{array}{l}A \\
B \\
C\end{array}$ & - & - & - & 0.375 & $\begin{array}{c}3.5 \\
(1.75)\end{array}$ & 0.44 & 3 & 2.37 & A8 \\
\hline & (3@5)8-8-T9.5-2\#3-i-2.5-3-14.5 & $\begin{array}{l}A \\
B \\
C\end{array}$ & 4 & $\begin{array}{c}6 \\
(4.5)\end{array}$ & 0.22 & 0.375 & $\begin{array}{c}3.5 \\
(1.75)\end{array}$ & 0.44 & 3 & 2.37 & A8 \\
\hline & (3@7)8-8-T9.5-0-i-2.5-3-14.5 & $\begin{array}{l}\text { A } \\
B \\
C\end{array}$ & - & - & - & 0.5 & $\begin{array}{c}4.5 \\
(2.25)\end{array}$ & 0.80 & 3 & 2.37 & A8 \\
\hline & (3@7)8-8-T9.5-2\#3-i-2.5-3-14.5 & $\begin{array}{l}A \\
B \\
C\end{array}$ & 4 & $\begin{array}{c}6 \\
(4.5)\end{array}$ & 0.22 & 0.5 & $\begin{array}{c}4.5 \\
(2.25)\end{array}$ & 0.80 & 3 & 2.37 & A8 \\
\hline
\end{tabular}

\footnotetext{
${ }^{*}$ Value in parenthesis is the spacing between the first hoop and the center of the headed bar
} 
Table A.2 Cont. Comprehensive test results and data for beam-column joint specimens

\begin{tabular}{|c|c|c|c|c|c|c|c|c|c|c|}
\hline & Specimen & Head & $\begin{array}{l}\text { Failure } \\
\text { Type }\end{array}$ & $\begin{array}{c}\text { Lead (Head) } \\
\text { Slip } \\
\text { in. }\end{array}$ & $\begin{array}{l}\boldsymbol{T}_{\max } \\
\text { kips }\end{array}$ & $\begin{array}{c}\boldsymbol{f}_{\text {su,max }} \\
\mathrm{ksi}\end{array}$ & $\begin{array}{l}\boldsymbol{T}_{\text {ind }} \\
\text { kips }\end{array}$ & $\begin{array}{l}\boldsymbol{T}_{\text {total }} \\
\text { kips }\end{array}$ & $\begin{array}{c}\boldsymbol{T} \\
\text { kips }\end{array}$ & $\begin{array}{l}\boldsymbol{f}_{\mathrm{su}} \\
\mathrm{ksi}\end{array}$ \\
\hline \multirow{15}{*}{ Group 9} & 8-8-T9.5-0-i-2.5-3-9.5 & $\begin{array}{l}\text { A } \\
\text { B }\end{array}$ & CB & $\begin{array}{l}0.168 \\
0.127\end{array}$ & $\begin{array}{l}65.0 \\
65.5\end{array}$ & $\begin{array}{l}82.3 \\
82.9\end{array}$ & $\begin{array}{l}65.0 \\
65.5\end{array}$ & 130.5 & 65.2 & 82.5 \\
\hline & 8-8-T9.5-2\#3-i-2.5-3-9.5 & $\begin{array}{l}\mathrm{A} \\
\mathrm{B}\end{array}$ & CB & $\begin{array}{c}- \\
0.103(0.003)\end{array}$ & $\begin{array}{l}69.0 \\
68.5\end{array}$ & $\begin{array}{l}87.3 \\
86.7 \\
\end{array}$ & $\begin{array}{l}69.0 \\
68.5 \\
\end{array}$ & 137.5 & 68.7 & 87.0 \\
\hline & (3@4)8-8-T9.5-0-i-2.5-3-9.5 & $\begin{array}{l}\text { A } \\
\text { B } \\
\text { C }\end{array}$ & CB & $\begin{array}{c}0.421 \\
0.232 \\
0.356(0.098)\end{array}$ & $\begin{array}{l}33.5 \\
43.2 \\
44.2 \\
\end{array}$ & $\begin{array}{l}42.4 \\
54.7 \\
55.9 \\
\end{array}$ & $\begin{array}{l}33.5 \\
43.2 \\
44.1 \\
\end{array}$ & 120.8 & 40.3 & 51.0 \\
\hline & (3@4)8-8-T9.5-2\#3-i-2.5-3-9.5 & $\begin{array}{l}\mathrm{A} \\
\mathrm{B} \\
\mathrm{C}\end{array}$ & CB & $\begin{array}{c}0.440 \\
0.293 \\
0.230(0.051) \\
\end{array}$ & $\begin{array}{l}51.5 \\
54.5 \\
49.3 \\
\end{array}$ & $\begin{array}{l}65.2 \\
69.0 \\
62.4 \\
\end{array}$ & $\begin{array}{l}51.5 \\
54.5 \\
49.3 \\
\end{array}$ & 155.3 & 51.8 & 65.6 \\
\hline & (3@5)8-8-T9.5-0-i-2.5-3-9.5 & $\begin{array}{l}\mathrm{A} \\
\mathrm{B} \\
\mathrm{C}\end{array}$ & CB & $\begin{array}{c}- \\
- \\
0.015(0.055) \\
\end{array}$ & $\begin{array}{l}54.5 \\
27.9 \\
51.0 \\
\end{array}$ & $\begin{array}{l}69.0 \\
35.3 \\
64.6 \\
\end{array}$ & $\begin{array}{l}54.5 \\
27.9 \\
51.0 \\
\end{array}$ & 133.5 & 44.5 & 56.3 \\
\hline & (3@5)8-8-T9.5-2\#3-i-2.5-3-9.5 & $\begin{array}{l}\mathrm{A} \\
\mathrm{B} \\
\mathrm{C}\end{array}$ & CB & $\begin{array}{c}0.373 \\
0.430 \\
0.342(0.001) \\
\end{array}$ & $\begin{array}{l}55.7 \\
60.6 \\
52.0 \\
\end{array}$ & $\begin{array}{l}70.5 \\
76.7 \\
65.8 \\
\end{array}$ & $\begin{array}{l}55.2 \\
60.6 \\
52.0 \\
\end{array}$ & 167.8 & 55.9 & 70.8 \\
\hline & (3@7)8-8-T9.5-0-i-2.5-3-9.5 & $\begin{array}{l}\text { A } \\
\text { B } \\
\text { C }\end{array}$ & CB & $\begin{array}{c}- \\
0.180 \\
0.094(0.008)\end{array}$ & $\begin{array}{l}54.2 \\
66.3 \\
85.6 \\
\end{array}$ & $\begin{array}{c}68.6 \\
83.9 \\
108.4 \\
\end{array}$ & $\begin{array}{l}54.2 \\
66.3 \\
85.6 \\
\end{array}$ & 206.1 & 68.7 & 87.0 \\
\hline & (3@7)8-8-T9.5-2\#3-i-2.5-3-9.5 & $\begin{array}{l}\mathrm{A} \\
\mathrm{B} \\
\mathrm{C}\end{array}$ & CB & $\begin{array}{c}0.469 \\
0.124 \\
0.145(0.011) \\
\end{array}$ & $\begin{array}{l}65.7 \\
62.6 \\
75.1 \\
\end{array}$ & $\begin{array}{l}83.2 \\
79.2 \\
95.1 \\
\end{array}$ & $\begin{array}{l}65.2 \\
62.6 \\
75.1 \\
\end{array}$ & 202.9 & 67.6 & 85.6 \\
\hline & 8-8-T9.5-0-i-2.5-3-14.5 & $\begin{array}{l}\mathrm{A} \\
\mathrm{B}\end{array}$ & Y & $\begin{array}{c}- \\
0.038 \\
\end{array}$ & $\begin{array}{l}117.3 \\
120.3 \\
\end{array}$ & $\begin{array}{l}148.5 \\
152.3 \\
\end{array}$ & $\begin{array}{l}117.3 \\
120.3 \\
\end{array}$ & 237.6 & 118.8 & 150.4 \\
\hline & (3@4)8-8-T9.5-0-i-2.5-3-14.5 & $\begin{array}{l}\text { A } \\
\text { B } \\
\text { C }\end{array}$ & CB & $\begin{array}{c}- \\
- \\
0.073 \\
\end{array}$ & $\begin{array}{l}80.9 \\
79.2 \\
75.0 \\
\end{array}$ & $\begin{array}{c}102.4 \\
100.3 \\
94.9 \\
\end{array}$ & $\begin{array}{l}80.9 \\
79.2 \\
69.7 \\
\end{array}$ & 229.7 & 76.6 & 97.0 \\
\hline & (3@4)8-8-T9.5-2\#3-i-2.5-3-14.5 & $\begin{array}{l}\mathrm{A} \\
\mathrm{B} \\
\mathrm{C}\end{array}$ & CB & $\begin{array}{c}0.122 \\
- \\
0.165(0.016) \\
\end{array}$ & $\begin{array}{l}79.5 \\
89.3 \\
87.5 \\
\end{array}$ & $\begin{array}{l}100.6 \\
113.0 \\
110.8 \\
\end{array}$ & $\begin{array}{l}79.5 \\
89.3 \\
87.5 \\
\end{array}$ & 256.3 & 85.4 & 108.1 \\
\hline & (3@5)8-8-T9.5-0-i-2.5-3-14.5 & $\begin{array}{l}\mathrm{A} \\
\mathrm{B} \\
\mathrm{C}\end{array}$ & CB & $\begin{array}{c}0.086 \\
- \\
0.090(0.031)\end{array}$ & $\begin{array}{c}87.3 \\
104.0 \\
88.5 \\
\end{array}$ & $\begin{array}{l}110.5 \\
131.6 \\
112.0 \\
\end{array}$ & $\begin{array}{c}87.0 \\
104.0 \\
88.5 \\
\end{array}$ & 279.6 & 93.2 & 118.0 \\
\hline & (3@5)8-8-T9.5-2\#3-i-2.5-3-14.5 & $\begin{array}{l}\text { A } \\
\text { B } \\
\text { C }\end{array}$ & CB & $\begin{array}{c}0.144 \\
- \\
0.083 \\
\end{array}$ & $\begin{array}{c}93.8 \\
99.3 \\
122.5 \\
\end{array}$ & $\begin{array}{l}118.7 \\
125.7 \\
155.1 \\
\end{array}$ & $\begin{array}{c}93.7 \\
99.3 \\
122.5 \\
\end{array}$ & 315.5 & 105.2 & 133.2 \\
\hline & (3@7)8-8-T9.5-0-i-2.5-3-14.5 & $\begin{array}{l}\text { A } \\
\text { B } \\
\text { C }\end{array}$ & $\mathrm{CB} / \mathrm{BS}$ & $\begin{array}{l}0.138 \\
0.166 \\
0.130 \\
\end{array}$ & $\begin{array}{c}104.4 \\
99.2 \\
108.3 \\
\end{array}$ & $\begin{array}{l}132.2 \\
125.6 \\
137.1 \\
\end{array}$ & $\begin{array}{c}104.4 \\
99.2 \\
108.3 \\
\end{array}$ & 311.9 & 104.0 & 131.6 \\
\hline & (3@7)8-8-T9.5-2\#3-i-2.5-3-14.5 & $\begin{array}{l}\text { A } \\
\text { B } \\
\text { C }\end{array}$ & CB & $\begin{array}{c}- \\
- \\
(0.027)\end{array}$ & $\begin{array}{c}105.8 \\
98.7 \\
136.6\end{array}$ & $\begin{array}{l}133.9 \\
124.9 \\
172.9\end{array}$ & $\begin{array}{c}105.8 \\
97.9 \\
136.6\end{array}$ & 340.3 & 113.4 & 143.5 \\
\hline
\end{tabular}


Table A.2 Cont. Comprehensive test results and data for beam-column joint specimens

\begin{tabular}{|c|c|c|c|c|c|c|c|c|c|c|}
\hline & Specimen & Head & $\begin{array}{l}\boldsymbol{c}_{\boldsymbol{o}} \\
\text { in. }\end{array}$ & $A_{b r g}$ & $\begin{array}{l}\ell \text { eh } \\
\text { in. }\end{array}$ & $\begin{array}{c}\ell \text { eh,avg } \\
\text { in. }\end{array}$ & $\begin{array}{l}\boldsymbol{f}_{\boldsymbol{c m}} \\
\text { psi }\end{array}$ & $\begin{array}{l}\text { Age } \\
\text { days }\end{array}$ & $\begin{array}{l}\boldsymbol{d}_{\boldsymbol{b}} \\
\text { in. }\end{array}$ & $\begin{array}{c}\boldsymbol{A}_{\boldsymbol{b}} \\
\text { in. }^{2}\end{array}$ \\
\hline \multirow{12}{*}{ Group 10} & (2@9)8-12-F4.1-0-i-2.5-3-12 & $\begin{array}{l}\mathrm{A} \\
\mathrm{B}\end{array}$ & 2.0 & $4.1 A_{b}$ & $\begin{array}{l}12.13 \\
12.00 \\
\end{array}$ & 12.06 & 12080 & 57 & 1 & 0.79 \\
\hline & (2@9)8-12-F9.1-0-i-2.5-3-12 & $\begin{array}{l}A \\
B\end{array}$ & 2.0 & $9.1 A_{b}$ & $\begin{array}{l}11.75 \\
12.00\end{array}$ & 11.88 & 12080 & 57 & 1 & 0.79 \\
\hline & (2@9)8-12-F4.1-5\#3-i-2.5-3-12 & $\begin{array}{l}A \\
B\end{array}$ & 2.0 & $4.1 A_{b}$ & $\begin{array}{l}11.94 \\
12.00 \\
\end{array}$ & 11.97 & 12080 & 57 & 1 & 0.79 \\
\hline & (2@9)8-12-F9.1-5\#3-i-2.5-3-12 & $\begin{array}{l}A \\
B\end{array}$ & 2.0 & $9.1 A_{b}$ & $\begin{array}{l}12.13 \\
12.13\end{array}$ & 12.13 & 12080 & 57 & 1 & 0.79 \\
\hline & (3@4.5)8-12-F4.1-0-i-2.5-3-12 & $\begin{array}{l}\text { A } \\
B \\
C\end{array}$ & 2.0 & $4.1 A_{b}$ & $\begin{array}{l}12.13 \\
12.25 \\
12.25 \\
\end{array}$ & 12.21 & 12040 & 58 & 1 & 0.79 \\
\hline & (3@4.5)8-12-F9.1-0-i-2.5-3-12 & $\begin{array}{l}\text { A } \\
B \\
C\end{array}$ & 2.0 & $9.1 A_{b}$ & $\begin{array}{l}12.00 \\
12.13 \\
12.00 \\
\end{array}$ & 12.04 & 12040 & 58 & 1 & 0.79 \\
\hline & (3@4.5)8-12-F4.1-5\#3-i-2.5-3-12 & $\begin{array}{l}\text { A } \\
\text { B } \\
\text { C }\end{array}$ & 2.0 & $4.1 A_{b}$ & $\begin{array}{l}12.13 \\
12.19 \\
12.19\end{array}$ & 12.17 & 12040 & 58 & 1 & 0.79 \\
\hline & (3@4.5)8-12-F9.1-5\#3-i-2.5-3-12 & $\begin{array}{l}\text { A } \\
B \\
C\end{array}$ & 2.0 & $9.1 A_{b}$ & $\begin{array}{l}11.94 \\
11.88 \\
11.88\end{array}$ & 11.90 & 12040 & 58 & 1 & 0.79 \\
\hline & (4@3)8-12-F4.1-0-i-2.5-3-12 & $\begin{array}{l}\text { A } \\
B \\
C \\
D\end{array}$ & 2.0 & $4.1 A_{b}$ & $\begin{array}{l}12.00 \\
12.00 \\
12.00 \\
12.00\end{array}$ & 12.00 & 12040 & 58 & 1 & 0.79 \\
\hline & (4@3)8-12-F9.1-0-i-2.5-3-12 & $\begin{array}{l}\text { A } \\
B \\
C \\
D\end{array}$ & 2.0 & $9.1 A_{b}$ & $\begin{array}{l}12.06 \\
12.13 \\
12.25 \\
12.25\end{array}$ & 12.17 & 12360 & 61 & 1 & 0.79 \\
\hline & (4@3)8-12-F4.1-5\#3-i-2.5-3-12 & $\begin{array}{l}\mathrm{A} \\
\mathrm{B} \\
\mathrm{C} \\
\mathrm{D}\end{array}$ & 2.0 & $4.1 A_{b}$ & $\begin{array}{l}12.00 \\
12.00 \\
12.13 \\
12.00 \\
\end{array}$ & 12.03 & 12360 & 61 & 1 & 0.79 \\
\hline & (4@3)8-12-F9.1-5\#3-i-2.5-3-12 & $\begin{array}{l}\text { A } \\
B \\
C \\
D\end{array}$ & 2.0 & $9.1 A_{b}$ & $\begin{array}{l}12.00 \\
12.00 \\
12.00 \\
11.81 \\
\end{array}$ & 11.95 & 12360 & 61 & 1 & 0.79 \\
\hline \multirow{4}{*}{ Group 11} & 8-8-O4.5-0-i-2.5-3-9.5 & $\begin{array}{l}\text { A } \\
B\end{array}$ & 1.6 & $4.5 A_{b}$ & $\begin{array}{l}9.13 \\
9.25 \\
\end{array}$ & 9.19 & 6710 & 16 & 1 & 0.79 \\
\hline & (2@9)8-8-O4.5-0-i-2.5-3-9.5 & $\begin{array}{l}A \\
B\end{array}$ & 1.6 & $4.5 A_{b}$ & $\begin{array}{l}9.13 \\
8.88 \\
\end{array}$ & 9.00 & 6710 & 16 & 1 & 0.79 \\
\hline & (2@7)8-8-O4.5-0-i-2.5-3-9.5 & $\begin{array}{l}A \\
B\end{array}$ & 1.6 & $4.5 A_{b}$ & $\begin{array}{l}9.38 \\
9.13\end{array}$ & 9.25 & 6710 & 16 & 1 & 0.79 \\
\hline & (2@5)8-8-O4.5-0-i-2.5-3-9.5 & $\begin{array}{l}A \\
B\end{array}$ & 1.6 & $4.5 A_{b}$ & $\begin{array}{l}9.13 \\
8.88\end{array}$ & 9.00 & 6710 & 16 & 1 & 0.79 \\
\hline
\end{tabular}


Table A.2 Cont. Comprehensive test results and data for beam-column joint specimens

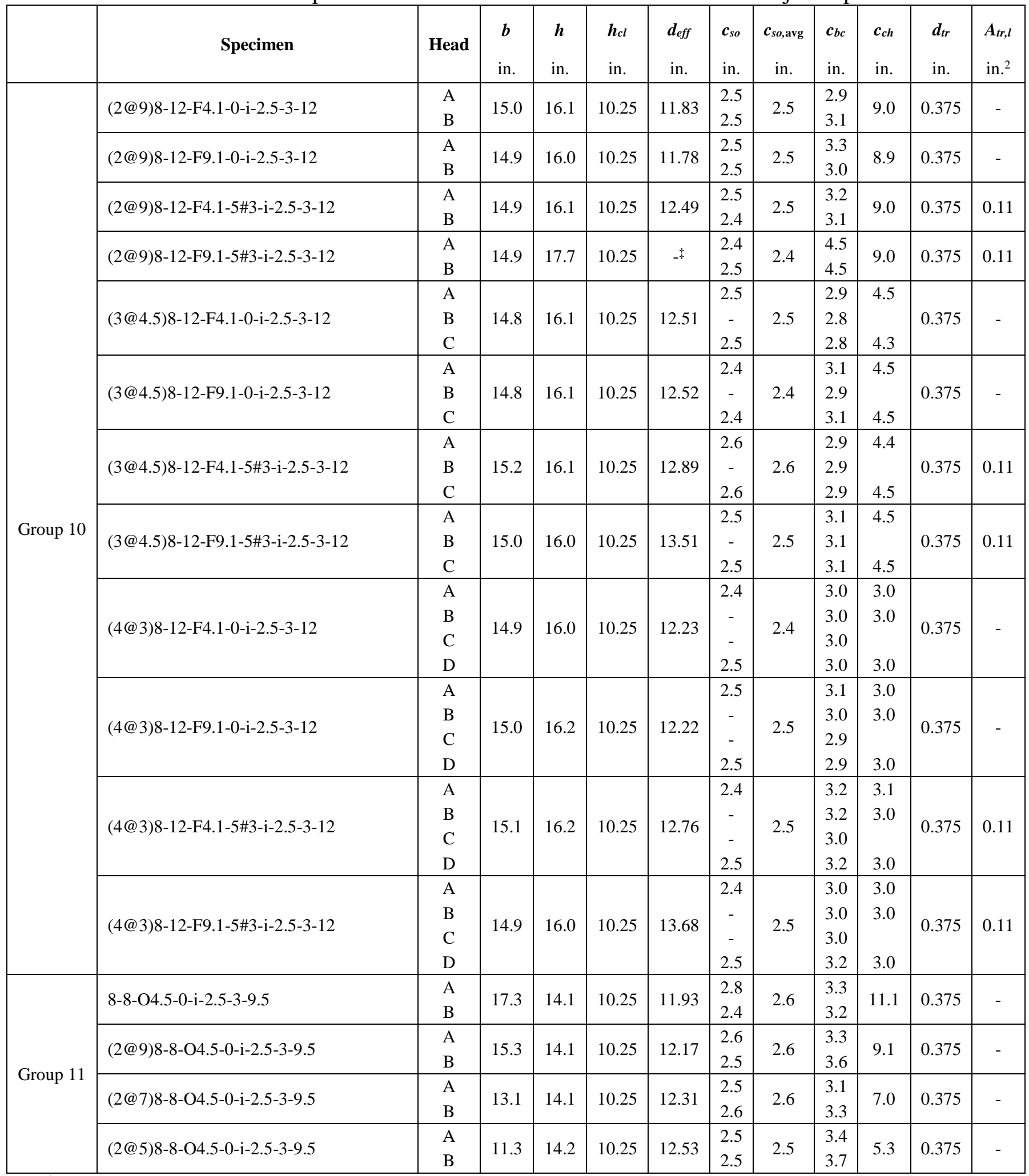

\footnotetext{
${ }^{\ddagger} d_{\text {eff }}$ was not calculated for specimen with bar yielding
} 
Table A.2 Cont. Comprehensive test results and data for beam-column joint specimens

\begin{tabular}{|c|c|c|c|c|c|c|c|c|c|c|c|}
\hline & Specimen & Head & $N$ & $\begin{array}{l}\boldsymbol{S}_{t r}{ }^{*} \\
\text { in. }\end{array}$ & $\begin{array}{l}\boldsymbol{A}_{\boldsymbol{t t}} \\
\text { in. }^{2}\end{array}$ & $\begin{array}{l}d_{\text {tro }} \\
\text { in. }\end{array}$ & $\begin{array}{c}S_{\text {tro }}{ }^{*} \\
\text { in. }\end{array}$ & $\begin{array}{l}\boldsymbol{A}_{\boldsymbol{a} \boldsymbol{b}} \\
\text { in. }^{2}\end{array}$ & $n$ & $\begin{array}{l}\boldsymbol{A}_{\boldsymbol{h s}} \\
\text { in. }^{2}\end{array}$ & $\begin{array}{l}\text { Long. } \\
\text { Reinf. } \\
\text { Layout }\end{array}$ \\
\hline \multirow{12}{*}{ Group 10} & (2@9)8-12-F4.1-0-i-2.5-3-12 & $\begin{array}{l}A \\
B\end{array}$ & - & - & - & 0.5 & $\begin{array}{c}3 \\
(1.5)\end{array}$ & 1.20 & 2 & 1.58 & A5 \\
\hline & (2@9)8-12-F9.1-0-i-2.5-3-12 & $\begin{array}{l}A \\
B\end{array}$ & - & - & - & 0.5 & $\begin{array}{c}3 \\
(1.5)\end{array}$ & 1.20 & 2 & 1.58 & A5 \\
\hline & (2@9)8-12-F4.1-5\#3-i-2.5-3-12 & $\begin{array}{l}A \\
B\end{array}$ & 10 & $\begin{array}{c}3 \\
(1.5)\end{array}$ & 0.66 & 0.5 & $\begin{array}{c}3 \\
(1.5)\end{array}$ & 1.20 & 2 & 1.58 & A5 \\
\hline & (2@9)8-12-F9.1-5\#3-i-2.5-3-12 & $\begin{array}{l}\text { A } \\
B\end{array}$ & 10 & $\begin{array}{c}3 \\
(1.5)\end{array}$ & 0.66 & 0.5 & $\begin{array}{c}3 \\
(1.5)\end{array}$ & 0.76 & 2 & 1.58 & A5 \\
\hline & (3@4.5)8-12-F4.1-0-i-2.5-3-12 & $\begin{array}{l}\text { A } \\
B \\
C\end{array}$ & - & - & - & 0.5 & $\begin{array}{c}3 \\
(1.5)\end{array}$ & 1.20 & 3 & 2.37 & A5 \\
\hline & (3@4.5)8-12-F9.1-0-i-2.5-3-12 & $\begin{array}{l}\text { A } \\
\text { B } \\
\text { C }\end{array}$ & - & - & - & 0.5 & $\begin{array}{c}3 \\
(1.5)\end{array}$ & 1.20 & 3 & 2.37 & A5 \\
\hline & (3@4.5)8-12-F4.1-5\#3-i-2.5-3-12 & $\begin{array}{l}\text { A } \\
B \\
C\end{array}$ & 10 & $\begin{array}{c}3 \\
(1.5)\end{array}$ & 0.66 & 0.5 & $\begin{array}{c}3 \\
(1.5)\end{array}$ & 1.20 & 3 & 2.37 & A5 \\
\hline & (3@4.5)8-12-F9.1-5\#3-i-2.5-3-12 & $\begin{array}{l}\text { A } \\
B \\
C\end{array}$ & 10 & $\begin{array}{c}3 \\
(1.5)\end{array}$ & 0.66 & 0.5 & $\begin{array}{c}3 \\
(1.5)\end{array}$ & 1.20 & 3 & 2.37 & A5 \\
\hline & (4@3)8-12-F4.1-0-i-2.5-3-12 & $\begin{array}{l}\text { A } \\
\text { B } \\
\text { C } \\
\text { D }\end{array}$ & - & - & - & 0.5 & $\begin{array}{c}3 \\
(1.5)\end{array}$ & 1.20 & 4 & 3.16 & A5 \\
\hline & (4@3)8-12-F9.1-0-i-2.5-3-12 & $\begin{array}{l}A \\
B \\
C \\
D\end{array}$ & - & - & - & 0.5 & $\begin{array}{c}3 \\
(1.5)\end{array}$ & 1.20 & 4 & 3.16 & A5 \\
\hline & (4@3)8-12-F4.1-5\#3-i-2.5-3-12 & $\begin{array}{l}\text { A } \\
\text { B } \\
\text { C } \\
\text { D }\end{array}$ & 10 & $\begin{array}{c}3 \\
(1.5)\end{array}$ & 0.66 & 0.5 & $\begin{array}{c}3 \\
(1.5)\end{array}$ & 1.20 & 4 & 3.16 & A5 \\
\hline & (4@3)8-12-F9.1-5\#3-i-2.5-3-12 & $\begin{array}{l}\text { A } \\
B \\
C \\
D\end{array}$ & 10 & $\begin{array}{c}3 \\
(1.5)\end{array}$ & 0.66 & 0.5 & $\begin{array}{c}3 \\
(1.5)\end{array}$ & 1.20 & 4 & 3.16 & A5 \\
\hline \multirow{4}{*}{ Group 11} & 8-8-O4.5-0-i-2.5-3-9.5 & $\begin{array}{l}A \\
B\end{array}$ & - & - & - & 0.375 & $\begin{array}{c}4 \\
(2) \\
\end{array}$ & 0.44 & 2 & 1.58 & A4 \\
\hline & (2@9)8-8-O4.5-0-i-2.5-3-9.5 & $\begin{array}{l}A \\
B\end{array}$ & - & - & - & 0.375 & $\begin{array}{c}4 \\
(2) \\
\end{array}$ & 0.44 & 2 & 1.58 & A4 \\
\hline & (2@7)8-8-O4.5-0-i-2.5-3-9.5 & $\begin{array}{l}A \\
B\end{array}$ & - & - & - & 0.375 & $\begin{array}{c}4 \\
(2)\end{array}$ & 0.44 & 2 & 1.58 & A4 \\
\hline & (2@5)8-8-O4.5-0-i-2.5-3-9.5 & $\begin{array}{l}A \\
B\end{array}$ & - & - & - & 0.375 & $\begin{array}{c}44 \\
(2)\end{array}$ & 0.44 & 2 & 1.58 & A4 \\
\hline
\end{tabular}

${ }^{*}$ Value in parenthesis is the spacing between the first hoop and the center of the headed bar 
Table A.2 Cont. Comprehensive test results and data for beam-column joint specimens

\begin{tabular}{|c|c|c|c|c|c|c|c|c|c|c|}
\hline & Specimen & Head & $\begin{array}{c}\text { Failure } \\
\text { Type }\end{array}$ & $\begin{array}{c}\text { Lead (Head) } \\
\text { Slip } \\
\text { in. }\end{array}$ & $\begin{array}{l}T_{\max } \\
\text { kips }\end{array}$ & $\begin{array}{c}\boldsymbol{f}_{\text {su,max }} \\
\text { ksi }\end{array}$ & $\begin{array}{l}\boldsymbol{T}_{\text {ind }} \\
\text { kips }\end{array}$ & $\begin{array}{l}\boldsymbol{T}_{\text {total }} \\
\text { kips }\end{array}$ & $\begin{array}{c}\boldsymbol{T} \\
\text { kips }\end{array}$ & $\begin{array}{l}f_{s u} \\
\mathrm{ksi}\end{array}$ \\
\hline \multirow{12}{*}{ Group 10} & (2@9)8-12-F4.1-0-i-2.5-3-12 & $\begin{array}{l}\mathrm{A} \\
\mathrm{B}\end{array}$ & $\mathrm{CB} / \mathrm{FP}$ & - & $\begin{array}{l}79.8 \\
78.3\end{array}$ & $\begin{array}{c}101.0 \\
99.1\end{array}$ & $\begin{array}{l}79.8 \\
78.3\end{array}$ & 158.1 & 79.1 & 100.1 \\
\hline & (2@9)8-12-F9.1-0-i-2.5-3-12 & $\begin{array}{l}\mathrm{A} \\
\mathrm{B}\end{array}$ & $\mathrm{CB} / \mathrm{BS}$ & $\begin{array}{c}0.048 \\
-\end{array}$ & $\begin{array}{l}76.1 \\
76.9\end{array}$ & $\begin{array}{l}96.3 \\
97.3\end{array}$ & $\begin{array}{l}76.1 \\
76.9\end{array}$ & 153.0 & 76.5 & 96.8 \\
\hline & (2@9)8-12-F4.1-5\#3-i-2.5-3-12 & $\begin{array}{l}\text { A } \\
\text { B }\end{array}$ & $\mathrm{SB} / \mathrm{FP}$ & $\begin{array}{l}0.126 \\
0.126\end{array}$ & $\begin{array}{l}112.5^{\ddagger} \\
111.3\end{array}$ & $\begin{array}{l}142.4^{\ddagger} \\
140.9\end{array}$ & $\begin{array}{l}112.5 \\
111.3\end{array}$ & 223.8 & 111.9 & 141.6 \\
\hline & (2@9)8-12-F9.1-5\#3-i-2.5-3-12 & $\begin{array}{l}\text { A } \\
B\end{array}$ & $\mathrm{Y}$ & $\begin{array}{l}0.200 \\
0.025\end{array}$ & $\begin{array}{l}125.2 \\
117.1\end{array}$ & $\begin{array}{l}158.5 \\
148.2\end{array}$ & $\begin{array}{l}125.2 \\
117.1\end{array}$ & 242.3 & 121.2 & 153.4 \\
\hline & (3@4.5)8-12-F4.1-0-i-2.5-3-12 & $\begin{array}{l}\text { A } \\
\text { B } \\
\text { C }\end{array}$ & CB & $\begin{array}{l}0.133 \\
0.037 \\
0.089\end{array}$ & $\begin{array}{l}79.1 \\
75.8 \\
70.7\end{array}$ & $\begin{array}{c}100.1 \\
95.9 \\
89.5\end{array}$ & $\begin{array}{l}79.1 \\
75.8 \\
70.7\end{array}$ & 225.7 & 75.2 & 95.2 \\
\hline & (3@4.5)8-12-F9.1-0-i-2.5-3-12 & $\begin{array}{l}\text { A } \\
\text { B } \\
\text { C }\end{array}$ & $\mathrm{CB}$ & $\begin{array}{c}(0.046) \\
- \\
0.117\end{array}$ & $\begin{array}{l}77.8 \\
63.3 \\
85.1\end{array}$ & $\begin{array}{c}98.5 \\
80.1 \\
107.7\end{array}$ & $\begin{array}{l}77.8 \\
63.3 \\
85.1\end{array}$ & 226.2 & 75.4 & 95.4 \\
\hline & (3@4.5)8-12-F4.1-5\#3-i-2.5-3-12 & $\begin{array}{l}\text { A } \\
\text { B } \\
\text { C }\end{array}$ & CB & $\begin{array}{l}0.170 \\
0.094 \\
0.169 \\
\end{array}$ & $\begin{array}{l}83.8 \\
86.0 \\
93.2 \\
\end{array}$ & $\begin{array}{l}106.1 \\
108.9 \\
118.0 \\
\end{array}$ & $\begin{array}{l}83.8 \\
86.0 \\
93.2 \\
\end{array}$ & 263.1 & 87.7 & 111.0 \\
\hline & (3@4.5)8-12-F9.1-5\#3-i-2.5-3-12 & $\begin{array}{l}\mathrm{A} \\
\mathrm{B} \\
\mathrm{C}\end{array}$ & CB & $\begin{array}{l}0.250 \\
0.096 \\
0.234\end{array}$ & $\begin{array}{l}108.1 \\
110.7 \\
106.9\end{array}$ & $\begin{array}{l}136.8 \\
140.1 \\
135.3\end{array}$ & $\begin{array}{l}108.1 \\
110.7 \\
106.9\end{array}$ & 325.7 & 108.6 & 137.4 \\
\hline & (4@3)8-12-F4.1-0-i-2.5-3-12 & $\begin{array}{l}\mathrm{A} \\
\mathrm{B} \\
\mathrm{C} \\
\mathrm{D}\end{array}$ & CB & $\begin{array}{c}- \\
- \\
0.135 \\
0.032 \\
\end{array}$ & $\begin{array}{l}41.7 \\
49.5 \\
66.8 \\
39.4 \\
\end{array}$ & $\begin{array}{l}52.8 \\
62.7 \\
84.6 \\
49.9 \\
\end{array}$ & $\begin{array}{l}41.7 \\
49.5 \\
66.8 \\
39.4 \\
\end{array}$ & 197.2 & 49.3 & 62.4 \\
\hline & (4@3)8-12-F9.1-0-i-2.5-3-12 & $\begin{array}{l}\text { A } \\
B \\
C \\
D\end{array}$ & CB & $\begin{array}{l}- \\
- \\
- \\
-\end{array}$ & $\begin{array}{l}49.2 \\
45.7 \\
53.2 \\
53.1 \\
\end{array}$ & $\begin{array}{l}62.3 \\
57.8 \\
67.3 \\
67.2 \\
\end{array}$ & $\begin{array}{l}49.2 \\
45.7 \\
53.2 \\
53.1 \\
\end{array}$ & 201.3 & 50.3 & 63.7 \\
\hline & (4@3)8-12-F4.1-5\#3-i-2.5-3-12 & $\begin{array}{l}\text { A } \\
B \\
C \\
D\end{array}$ & CB & $\begin{array}{c}0.030 \\
- \\
0.101 \\
0.093 \\
\end{array}$ & $\begin{array}{l}73.8 \\
63.3 \\
48.2 \\
71.5 \\
\end{array}$ & $\begin{array}{l}93.4 \\
80.1 \\
61.0 \\
90.5 \\
\end{array}$ & $\begin{array}{l}73.8 \\
63.3 \\
48.2 \\
71.5 \\
\end{array}$ & 256.7 & 64.2 & 81.2 \\
\hline & (4@3)8-12-F9.1-5\#3-i-2.5-3-12 & $\begin{array}{l}\text { A } \\
B \\
C \\
D\end{array}$ & CB & $\begin{array}{l}- \\
- \\
- \\
-\end{array}$ & $\begin{array}{c}85.2 \\
72.8 \\
111.1 \\
82.1 \\
\end{array}$ & $\begin{array}{c}107.8 \\
92.2 \\
140.6 \\
103.9 \\
\end{array}$ & $\begin{array}{c}85.2 \\
72.8 \\
111.1 \\
82.1 \\
\end{array}$ & 351.3 & 87.8 & 111.1 \\
\hline \multirow{4}{*}{ Group 11} & 8-8-O4.5-0-i-2.5-3-9.5 & $\begin{array}{l}\text { A } \\
\text { B }\end{array}$ & $\mathrm{CB} / \mathrm{FP}$ & $\begin{array}{l}0.002 \\
0.002\end{array}$ & $\begin{array}{l}61.9 \\
54.9\end{array}$ & $\begin{array}{l}78.4 \\
69.5\end{array}$ & $\begin{array}{l}61.8 \\
54.9\end{array}$ & 116.7 & 58.4 & 73.9 \\
\hline & (2@9)8-8-O4.5-0-i-2.5-3-9.5 & $\begin{array}{l}\text { A } \\
\text { B }\end{array}$ & CB & $\begin{array}{l}0.014 \\
0.019\end{array}$ & $\begin{array}{l}57.5 \\
60.1\end{array}$ & $\begin{array}{l}72.8 \\
76.1\end{array}$ & $\begin{array}{l}57.5 \\
60.1\end{array}$ & 117.6 & 58.8 & 74.4 \\
\hline & (2@7)8-8-O4.5-0-i-2.5-3-9.5 & $\begin{array}{l}\mathrm{A} \\
\mathrm{B}\end{array}$ & CB & $\begin{array}{l}0.010 \\
0.030 \\
\end{array}$ & $\begin{array}{l}57.2 \\
51.8 \\
\end{array}$ & $\begin{array}{l}72.4 \\
65.6 \\
\end{array}$ & $\begin{array}{l}57.2 \\
51.8\end{array}$ & 109.0 & 54.5 & 69.0 \\
\hline & (2@5)8-8-O4.5-0-i-2.5-3-9.5 & $\begin{array}{l}\mathrm{A} \\
\mathrm{B}\end{array}$ & CB & $\begin{array}{l}0.035 \\
0.041\end{array}$ & $\begin{array}{l}45.7 \\
56.7\end{array}$ & $\begin{array}{l}57.8 \\
71.8\end{array}$ & $\begin{array}{l}45.7 \\
56.7\end{array}$ & 102.4 & 51.2 & 64.8 \\
\hline
\end{tabular}

\footnotetext{
${ }^{\ddagger}$ No anchorage failure on the bar
} 
Table A.2 Cont. Comprehensive test results and data for beam-column joint specimens

\begin{tabular}{|c|c|c|c|c|c|c|c|c|c|c|}
\hline & Specimen & Head & $\begin{array}{l}\boldsymbol{c}_{o} \\
\text { in. }\end{array}$ & $A_{b r g}$ & $\begin{array}{l}\ell_{e h} \\
\text { in. }\end{array}$ & $\begin{array}{c}\ell \text { eh,avg } \\
\text { in. }\end{array}$ & $\begin{array}{l}\boldsymbol{f}_{\boldsymbol{c m}} \\
\text { psi }\end{array}$ & $\begin{array}{l}\text { Age } \\
\text { days }\end{array}$ & $\begin{array}{l}\boldsymbol{d}_{\boldsymbol{b}} \\
\text { in. }\end{array}$ & $\begin{array}{c}\boldsymbol{A}_{\boldsymbol{b}} \\
\text { in. }^{2}\end{array}$ \\
\hline \multirow{9}{*}{ Group 11} & (2@3)8-8-O4.5-0-i-2.5-3-9.5 & $\begin{array}{l}A \\
B\end{array}$ & 1.6 & $4.5 A_{b}$ & $\begin{array}{l}9.13 \\
8.88\end{array}$ & 9.00 & 6710 & 16 & 1 & 0.79 \\
\hline & (2@9)8-8-T4.0-0-i-2.5-3-9.5 & $\begin{array}{l}A \\
B\end{array}$ & 1.9 & $4.0 A_{b}$ & $\begin{array}{l}9.25 \\
9.50\end{array}$ & 9.38 & 6790 & 17 & 1 & 0.79 \\
\hline & (2@9)8-8-T4.0-5\#3-i-2.5-3-9.5 & $\begin{array}{l}A \\
B\end{array}$ & 1.9 & $4.0 A_{b}$ & $\begin{array}{l}9.50 \\
9.50\end{array}$ & 9.50 & 6790 & 17 & 1 & 0.79 \\
\hline & (3@4.5)8-8-T4.0-0-i-2.5-3-9.5 & $\begin{array}{l}\text { A } \\
B \\
C\end{array}$ & 1.9 & $4.0 A_{b}$ & $\begin{array}{l}9.25 \\
9.50 \\
9.25\end{array}$ & 9.33 & 6790 & 17 & 1 & 0.79 \\
\hline & (3@4.5)8-8-T4.0-5\#3-i-2.5-3-9.5 & $\begin{array}{l}\text { A } \\
B \\
C\end{array}$ & 1.9 & $4.0 A_{b}$ & $\begin{array}{l}9.13 \\
9.25 \\
9.13\end{array}$ & 9.17 & 6650 & 20 & 1 & 0.79 \\
\hline & (4@3)8-8-T4.0-0-i-2.5-3-9.5 & $\begin{array}{l}\text { A } \\
B \\
C \\
D\end{array}$ & 1.9 & $4.0 A_{b}$ & $\begin{array}{l}9.63 \\
9.63 \\
9.25 \\
9.38 \\
\end{array}$ & 9.47 & 6650 & 20 & 1 & 0.79 \\
\hline & (4@3)8-8-T4.0-5\#3-i-2.5-3-9.5 & $\begin{array}{l}\text { A } \\
B \\
C \\
D\end{array}$ & 1.9 & $4.0 A_{b}$ & $\begin{array}{l}9.75 \\
9.63 \\
9.88 \\
9.38\end{array}$ & 9.66 & 6650 & 20 & 1 & 0.79 \\
\hline & (3@3)8-8-T4.0-0-i-2.5-3-9.5 & $\begin{array}{l}\text { A } \\
\text { B } \\
\text { C }\end{array}$ & 1.9 & $4.0 A_{b}$ & $\begin{array}{l}9.25 \\
9.63 \\
9.50 \\
\end{array}$ & 9.46 & 6790 & 17 & 1 & 0.79 \\
\hline & (3@3)8-8-T4.0-5\#3-i-2.5-3-9.5 & $\begin{array}{l}\text { A } \\
B \\
C\end{array}$ & 1.9 & $4.0 A_{b}$ & $\begin{array}{l}9.25 \\
9.38 \\
9.38\end{array}$ & 9.33 & 6650 & 20 & 1 & 0.79 \\
\hline \multirow{7}{*}{ Group 12} & 8-5-F4.1-0-i-2.5-7-6 & $\begin{array}{l}A \\
B\end{array}$ & 2.0 & $4.1 A_{b}$ & $\begin{array}{l}6.06 \\
6.13\end{array}$ & 6.09 & 4930 & 14 & 1 & 0.79 \\
\hline & 8-5-F4.1-5\#3-i-2.5-7-6 & $\begin{array}{l}\text { A } \\
B\end{array}$ & 2.0 & $4.1 A_{b}$ & $\begin{array}{l}6.25 \\
6.25\end{array}$ & 6.25 & 4930 & 14 & 1 & 0.79 \\
\hline & (3@3)8-5-F4.1-0-i-2.5-7-6 & $\begin{array}{l}\mathrm{A} \\
\mathrm{B} \\
\mathrm{C}\end{array}$ & 2.0 & $4.1 A_{b}$ & $\begin{array}{l}6.06 \\
6.25 \\
6.25 \\
\end{array}$ & 6.19 & 4930 & 14 & 1 & 0.79 \\
\hline & (3@3)8-5-F4.1-5\#3-i-2.5-7-6 & $\begin{array}{l}\text { A } \\
B \\
C\end{array}$ & 2.0 & $4.1 A_{b}$ & $\begin{array}{l}6.00 \\
6.00 \\
6.00\end{array}$ & 6.00 & 4930 & 14 & 1 & 0.79 \\
\hline & (3@5)8-5-F4.1-0-i-2.5-7-6 & $\begin{array}{l}\text { A } \\
B \\
C\end{array}$ & 2.0 & $4.1 A_{b}$ & $\begin{array}{l}6.50 \\
6.25 \\
6.25 \\
\end{array}$ & 6.33 & 4930 & 14 & 1 & 0.79 \\
\hline & (3@5)8-5-F4.1-5\#3-i-2.5-7-6 & $\begin{array}{l}\text { A } \\
B \\
C\end{array}$ & 2.0 & $4.1 A_{b}$ & $\begin{array}{l}6.25 \\
6.13 \\
6.50\end{array}$ & 6.29 & 4930 & 14 & 1 & 0.79 \\
\hline & (3@7)8-5-F4.1-0-i-2.5-7-6 & $\begin{array}{l}A \\
B \\
C\end{array}$ & 2.0 & $4.1 A_{b}$ & $\begin{array}{l}6.25 \\
6.25 \\
6.25 \\
\end{array}$ & 6.25 & 4940 & 15 & 1 & 0.79 \\
\hline
\end{tabular}


Table A.2 Cont. Comprehensive test results and data for beam-column joint specimens

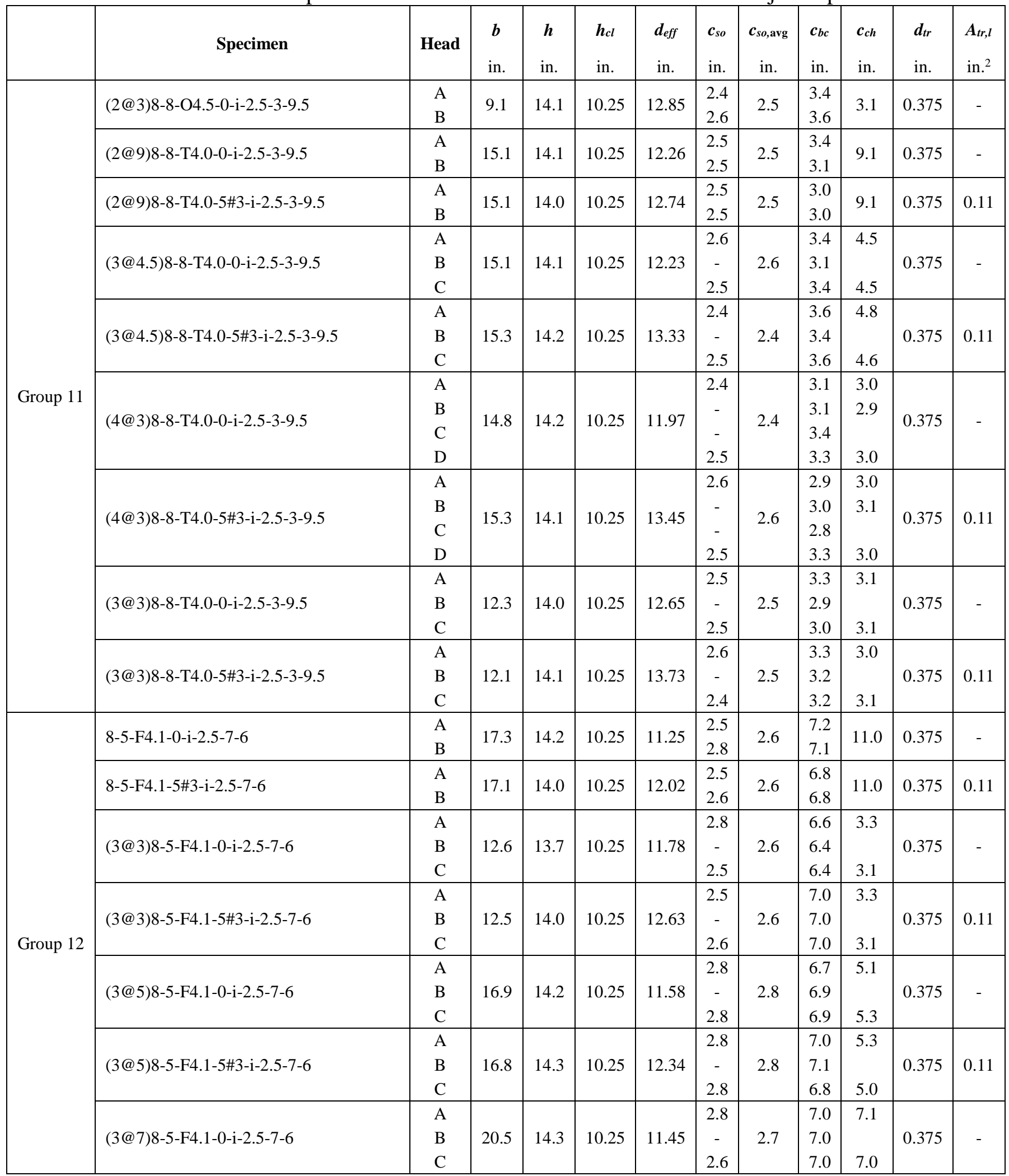


Table A.2 Cont. Comprehensive test results and data for beam-column joint specimens

\begin{tabular}{|c|c|c|c|c|c|c|c|c|c|c|c|}
\hline & Specimen & Head & $N$ & $\begin{array}{l}S_{t r}{ }^{*} \\
\text { in. }\end{array}$ & $\begin{array}{c}\boldsymbol{A}_{\boldsymbol{t t}} \\
\text { in. }^{2}\end{array}$ & $\begin{array}{l}\boldsymbol{d}_{\text {tro }} \\
\text { in. }\end{array}$ & $\begin{array}{c}\text { Stro }^{*} \\
\text { in. }\end{array}$ & $\begin{array}{l}\boldsymbol{A}_{\boldsymbol{a} \boldsymbol{b}} \\
\text { in. }^{2}\end{array}$ & $n$ & $\begin{array}{l}\boldsymbol{A}_{\boldsymbol{h s}} \\
\text { in. }^{2}\end{array}$ & $\begin{array}{l}\text { Long. } \\
\text { Reinf. } \\
\text { Layout }\end{array}$ \\
\hline \multirow{9}{*}{ Group 11} & (2@3)8-8-O4.5-0-i-2.5-3-9.5 & $\begin{array}{l}\text { A } \\
B\end{array}$ & - & - & - & 0.375 & $\begin{array}{c}4 \\
(2) \\
\end{array}$ & 0.44 & 2 & 1.58 & A4 \\
\hline & (2@9)8-8-T4.0-0-i-2.5-3-9.5 & $\begin{array}{l}\text { A } \\
B\end{array}$ & - & - & - & 0.5 & $\begin{array}{c}4 \\
(2)\end{array}$ & 0.80 & 2 & 1.58 & A9 \\
\hline & (2@9)8-8-T4.0-5\#3-i-2.5-3-9.5 & $\begin{array}{l}\mathrm{A} \\
\mathrm{B}\end{array}$ & 10 & $\begin{array}{c}3 \\
(1.5) \\
\end{array}$ & 0.66 & 0.5 & $\begin{array}{c}4 \\
(2) \\
\end{array}$ & 0.80 & 2 & 1.58 & A5 \\
\hline & (3@4.5)8-8-T4.0-0-i-2.5-3-9.5 & $\begin{array}{l}\mathrm{A} \\
\mathrm{B} \\
\mathrm{C}\end{array}$ & - & - & - & 0.5 & $\begin{array}{c}4 \\
(2)\end{array}$ & 0.80 & 3 & 2.37 & A9 \\
\hline & (3@4.5)8-8-T4.0-5\#3-i-2.5-3-9.5 & $\begin{array}{l}\text { A } \\
B \\
C\end{array}$ & 10 & $\begin{array}{c}3 \\
(1.5)\end{array}$ & 0.66 & 0.5 & $\begin{array}{c}4 \\
(2)\end{array}$ & 0.80 & 3 & 2.37 & A5 \\
\hline & (4@3)8-8-T4.0-0-i-2.5-3-9.5 & $\begin{array}{l}A \\
B \\
C \\
D\end{array}$ & - & - & - & 0.5 & $\begin{array}{c}4 \\
(2)\end{array}$ & 0.80 & 4 & 3.16 & A9 \\
\hline & (4@3)8-8-T4.0-5\#3-i-2.5-3-9.5 & $\begin{array}{l}\mathrm{A} \\
\mathrm{B} \\
\mathrm{C} \\
\mathrm{D}\end{array}$ & 10 & $\begin{array}{c}3 \\
(1.5)\end{array}$ & 0.66 & 0.5 & $\begin{array}{c}4 \\
(2)\end{array}$ & 0.80 & 4 & 3.16 & A5 \\
\hline & (3@3)8-8-T4.0-0-i-2.5-3-9.5 & $\begin{array}{l}\text { A } \\
\text { B } \\
\text { C }\end{array}$ & - & - & - & 0.375 & $\begin{array}{c}3 \\
(1.5)\end{array}$ & 0.66 & 3 & 2.37 & A5 \\
\hline & (3@3)8-8-T4.0-5\#3-i-2.5-3-9.5 & $\begin{array}{l}A \\
B \\
C \\
\end{array}$ & 10 & $\begin{array}{c}3 \\
(1.5)\end{array}$ & 0.66 & 0.375 & $\begin{array}{c}3 \\
(1.5)\end{array}$ & 0.66 & 3 & 2.37 & A5 \\
\hline \multirow{7}{*}{ Group 12} & 8-5-F4.1-0-i-2.5-7-6 & $\begin{array}{l}\mathrm{A} \\
\mathrm{B}\end{array}$ & - & - & - & 0.375 & $\begin{array}{c}3 \\
(1.5) \\
\end{array}$ & 0.66 & 2 & 1.58 & A4 \\
\hline & 8-5-F4.1-5\#3-i-2.5-7-6 & $\begin{array}{l}\text { A } \\
\text { B }\end{array}$ & 10 & $\begin{array}{c}3 \\
(1.5) \\
\end{array}$ & 0.66 & 0.375 & $\begin{array}{c}3 \\
(1.5) \\
\end{array}$ & 0.66 & 2 & 1.58 & A4 \\
\hline & (3@3)8-5-F4.1-0-i-2.5-7-6 & $\begin{array}{l}\text { A } \\
\text { B } \\
\text { C }\end{array}$ & - & - & - & 0.375 & $\begin{array}{c}3 \\
(1.5)\end{array}$ & 0.66 & 3 & 2.37 & A4 \\
\hline & (3@3)8-5-F4.1-5\#3-i-2.5-7-6 & $\begin{array}{l}\text { A } \\
\text { B } \\
\text { C }\end{array}$ & 10 & $\begin{array}{c}3 \\
(1.5)\end{array}$ & 0.66 & 0.375 & $\begin{array}{c}3 \\
(1.5)\end{array}$ & 0.66 & 3 & 2.37 & A5 \\
\hline & (3@5)8-5-F4.1-0-i-2.5-7-6 & $\begin{array}{l}\mathrm{A} \\
\mathrm{B} \\
\mathrm{C}\end{array}$ & - & - & - & 0.375 & $\begin{array}{c}3 \\
(1.5)\end{array}$ & 0.66 & 3 & 2.37 & A5 \\
\hline & (3@5)8-5-F4.1-5\#3-i-2.5-7-6 & $\begin{array}{l}\mathrm{A} \\
\mathrm{B} \\
\mathrm{C}\end{array}$ & 10 & $\begin{array}{c}3 \\
(1.5)\end{array}$ & 0.66 & 0.375 & $\begin{array}{c}3 \\
(1.5)\end{array}$ & 0.66 & 3 & 2.37 & A5 \\
\hline & (3@7)8-5-F4.1-0-i-2.5-7-6 & $\begin{array}{l}\text { A } \\
B \\
C\end{array}$ & - & - & - & 0.375 & $\begin{array}{c}3 \\
(1.5)\end{array}$ & 0.66 & 3 & 2.37 & A8 \\
\hline
\end{tabular}

${ }^{*}$ Value in parenthesis is the spacing between the first hoop and the center of the headed bar 
Table A.2 Cont. Comprehensive test results and data for beam-column joint specimens

\begin{tabular}{|c|c|c|c|c|c|c|c|c|c|c|}
\hline & Specimen & Head & $\begin{array}{c}\text { Failure } \\
\text { Type }\end{array}$ & $\begin{array}{c}\text { Lead (Head) } \\
\text { Slip } \\
\text { in. }\end{array}$ & $\begin{array}{l}\boldsymbol{T}_{\max } \\
\text { kips }\end{array}$ & $\begin{array}{c}\boldsymbol{f}_{\text {su, max }} \\
\mathrm{ksi}\end{array}$ & $\begin{array}{r}T_{\text {ind }} \\
\text { kips }\end{array}$ & $\begin{array}{l}\boldsymbol{T}_{\text {total }} \\
\text { kips }\end{array}$ & $\begin{array}{c}\boldsymbol{T} \\
\text { kips }\end{array}$ & $\begin{array}{l}f_{s u} \\
\text { ksi }\end{array}$ \\
\hline \multirow{9}{*}{ Group 11} & (2@3)8-8-04.5-0-i-2.5-3-9.5 & $\begin{array}{l}\text { A } \\
\text { B }\end{array}$ & СВ & $\begin{array}{l}0.037 \\
0.021\end{array}$ & $\begin{array}{l}51.9 \\
43.6\end{array}$ & $\begin{array}{l}65.7 \\
55.2\end{array}$ & $\begin{array}{l}51.9 \\
43.6\end{array}$ & 95.5 & 47.7 & 60.4 \\
\hline & (2@9)8-8-T4.0-0-i-2.5-3-9.5 & $\begin{array}{l}\text { A } \\
\text { B }\end{array}$ & $\mathrm{CB}$ & $\begin{array}{l}0.015 \\
0.016\end{array}$ & $\begin{array}{l}65.0 \\
58.7\end{array}$ & $\begin{array}{l}82.3 \\
74.3\end{array}$ & $\begin{array}{l}65.0 \\
58.7\end{array}$ & 123.7 & 61.8 & 78.2 \\
\hline & (2@9)8-8-T4.0-5\#3-i-2.5-3-9.5 & $\begin{array}{l}\text { A } \\
\text { B }\end{array}$ & $\mathrm{SB} / \mathrm{FP}$ & $\begin{array}{l}0.078 \\
0.035\end{array}$ & $\begin{array}{l}70.7 \\
87.5 \\
\end{array}$ & $\begin{array}{c}89.5 \\
110.8 \\
\end{array}$ & $\begin{array}{l}70.7 \\
82.6 \\
\end{array}$ & 153.3 & 76.7 & 97.1 \\
\hline & (3@4.5)8-8-T4.0-0-i-2.5-3-9.5 & $\begin{array}{l}\text { A } \\
\text { B } \\
\text { C }\end{array}$ & СВ & $\begin{array}{l}0.013 \\
0.013 \\
0.013\end{array}$ & $\begin{array}{l}43.9 \\
27.9 \\
50.3 \\
\end{array}$ & $\begin{array}{l}55.6 \\
35.3 \\
63.7 \\
\end{array}$ & $\begin{array}{l}43.9 \\
27.9 \\
50.3 \\
\end{array}$ & 122.1 & 40.7 & 51.5 \\
\hline & (3@4.5)8-8-T4.0-5\#3-i-2.5-3-9.5 & $\begin{array}{l}\text { A } \\
\text { B } \\
\text { C }\end{array}$ & $\mathrm{CB} / \mathrm{FP}$ & $\begin{array}{l}0.015 \\
0.558 \\
0.003\end{array}$ & $\begin{array}{l}56.5 \\
68.6 \\
66.4\end{array}$ & $\begin{array}{l}71.5 \\
86.8 \\
84.1\end{array}$ & $\begin{array}{l}55.3 \\
65.8 \\
66.3\end{array}$ & 187.4 & 62.5 & 79.1 \\
\hline & (4@3)8-8-T4.0-0-i-2.5-3-9.5 & $\begin{array}{l}\text { A } \\
\text { B } \\
\text { C } \\
\text { D }\end{array}$ & СВ & $\begin{array}{c}- \\
- \\
- \\
0.005\end{array}$ & $\begin{array}{l}25.2 \\
31.2 \\
31.7 \\
16.6\end{array}$ & $\begin{array}{l}31.9 \\
39.5 \\
40.1 \\
21.0\end{array}$ & $\begin{array}{l}25.2 \\
31.2 \\
31.7 \\
16.5\end{array}$ & 104.6 & 26.2 & 33.1 \\
\hline & (4@3)8-8-T4.0-5\#3-i-2.5-3-9.5 & $\begin{array}{l}\text { A } \\
\text { B } \\
\text { C } \\
\text { D } \\
\end{array}$ & СВ & $\begin{array}{c}0.005 \\
- \\
- \\
0.015 \\
\end{array}$ & $\begin{array}{l}57.7 \\
30.1 \\
52.3 \\
54.4 \\
\end{array}$ & $\begin{array}{l}73.0 \\
38.1 \\
66.2 \\
68.9 \\
\end{array}$ & $\begin{array}{l}57.7 \\
30.1 \\
52.3 \\
54.4 \\
\end{array}$ & 194.6 & 48.6 & 61.5 \\
\hline & (3@3)8-8-T4.0-0-i-2.5-3-9.5 & $\begin{array}{l}\text { A } \\
\text { B } \\
\text { C } \\
\end{array}$ & СВ & $\begin{array}{l}0.014 \\
0.016 \\
0.014 \\
\end{array}$ & $\begin{array}{l}39.9 \\
44.3 \\
33.9 \\
\end{array}$ & $\begin{array}{l}50.5 \\
56.1 \\
42.9 \\
\end{array}$ & $\begin{array}{l}39.9 \\
44.3 \\
33.9 \\
\end{array}$ & 118.1 & 39.4 & 49.8 \\
\hline & (3@3)8-8-T4.0-5\#3-i-2.5-3-9.5 & $\begin{array}{l}\text { A } \\
\text { B } \\
\text { C }\end{array}$ & СВ & $\begin{array}{c}0.003 \\
- \\
0.007\end{array}$ & $\begin{array}{l}56.9 \\
63.6 \\
49.3 \\
\end{array}$ & $\begin{array}{l}72.0 \\
80.5 \\
62.4 \\
\end{array}$ & $\begin{array}{l}56.8 \\
63.6 \\
49.2 \\
\end{array}$ & 169.6 & 56.5 & 71.6 \\
\hline \multirow{7}{*}{ Group 12} & 8-5-F4.1-0-i-2.5-7-6 & $\begin{array}{l}\text { A } \\
\text { B }\end{array}$ & СВ & $\begin{array}{l}0.005 \\
0.027\end{array}$ & $\begin{array}{l}30.2 \\
29.7 \\
\end{array}$ & $\begin{array}{l}38.2 \\
37.6\end{array}$ & $\begin{array}{l}27.7 \\
29.7 \\
\end{array}$ & 57.3 & 28.7 & 36.3 \\
\hline & $8-5-F 4.1-5 \# 3-i-2.5-7-6$ & $\begin{array}{l}\text { A } \\
\text { B }\end{array}$ & CB & $\begin{array}{l}0.027 \\
0.023\end{array}$ & $\begin{array}{l}51.6 \\
52.7\end{array}$ & $\begin{array}{l}65.3 \\
66.7\end{array}$ & $\begin{array}{l}48.8 \\
52.5\end{array}$ & 101.3 & 50.7 & 64.1 \\
\hline & (3@3)8-5-F4.1-0-i-2.5-7-6 & $\begin{array}{l}\text { A } \\
\text { B } \\
\text { C }\end{array}$ & $\mathrm{CB}$ & $\begin{array}{l}- \\
- \\
-\end{array}$ & $\begin{array}{l}15.5 \\
24.3 \\
22.7\end{array}$ & $\begin{array}{l}19.6 \\
30.8 \\
28.7\end{array}$ & $\begin{array}{l}14.9 \\
24.3 \\
22.7\end{array}$ & 61.8 & 20.6 & 26.1 \\
\hline & (3@3)8-5-F4.1-5\#3-i-2.5-7-6 & $\begin{array}{l}\text { A } \\
\text { B } \\
\text { C } \\
\end{array}$ & $\mathrm{CB}$ & $\begin{array}{l}- \\
- \\
-\end{array}$ & $\begin{array}{l}32.2 \\
30.8 \\
33.3\end{array}$ & $\begin{array}{l}40.8 \\
39.0 \\
42.2\end{array}$ & $\begin{array}{l}32.2 \\
30.8 \\
33.3\end{array}$ & 96.3 & 32.1 & 40.6 \\
\hline & (3@5)8-5-F4.1-0-i-2.5-7-6 & $\begin{array}{l}\text { A } \\
\text { B } \\
\text { C }\end{array}$ & СB & $\begin{array}{c}0.026 \\
- \\
0.002\end{array}$ & $\begin{array}{l}24.1 \\
23.8 \\
24.5 \\
\end{array}$ & $\begin{array}{l}30.5 \\
30.1 \\
31.0\end{array}$ & $\begin{array}{l}24.0 \\
23.3 \\
24.5 \\
\end{array}$ & 71.8 & 23.9 & 30.3 \\
\hline & (3@5)8-5-F4.1-5\#3-i-2.5-7-6 & $\begin{array}{l}\text { A } \\
\text { B } \\
\text { C }\end{array}$ & СВ & $\begin{array}{l}0.007 \\
0.014 \\
0.014\end{array}$ & $\begin{array}{l}31.3 \\
38.3 \\
43.8\end{array}$ & $\begin{array}{l}39.6 \\
48.5 \\
55.5\end{array}$ & $\begin{array}{l}30.9 \\
38.3 \\
43.4\end{array}$ & 112.6 & 37.5 & 47.5 \\
\hline & (3@7)8-5-F4.1-0-i-2.5-7-6 & $\begin{array}{l}\text { A } \\
\text { B } \\
\text { C }\end{array}$ & CB & $\begin{array}{c}0.001 \\
- \\
0.013\end{array}$ & $\begin{array}{l}31.1 \\
19.1 \\
31.1\end{array}$ & $\begin{array}{l}39.4 \\
24.2 \\
39.4\end{array}$ & $\begin{array}{l}31.1 \\
19.0 \\
31.1\end{array}$ & 81.2 & 27.1 & 34.3 \\
\hline
\end{tabular}


Table A.2 Cont. Comprehensive test results and data for beam-column joint specimens

\begin{tabular}{|c|c|c|c|c|c|c|c|c|c|c|}
\hline & Specimen & Head & $\begin{array}{l}\boldsymbol{c}_{\boldsymbol{o}} \\
\text { in. }\end{array}$ & $A_{b r g}$ & $\begin{array}{l}\ell_{e h} \\
\text { in. }\end{array}$ & $\begin{array}{c}\ell \text { eh, avg } \\
\text { in. }\end{array}$ & $\begin{array}{l}\boldsymbol{f}_{c m} \\
\text { psi }\end{array}$ & $\begin{array}{l}\text { Age } \\
\text { days }\end{array}$ & $\begin{array}{l}\boldsymbol{d}_{\boldsymbol{b}} \\
\text { in. }\end{array}$ & $\begin{array}{c}\boldsymbol{A}_{\boldsymbol{b}} \\
\text { in. }^{2}\end{array}$ \\
\hline \multirow{7}{*}{ Group 12} & (3@7)8-5-F4.1-5\#3-i-2.5-7-6 & $\begin{array}{l}\text { A } \\
B \\
C\end{array}$ & 2.0 & $4.1 A_{b}$ & $\begin{array}{l}6.00 \\
6.19 \\
6.13 \\
\end{array}$ & 6.10 & 4940 & 15 & 1 & 0.79 \\
\hline & 8-5-F9.1-0-i-2.5-7-6 & $\begin{array}{l}\text { A } \\
B\end{array}$ & 2.0 & $9.1 A_{b}$ & $\begin{array}{l}6.13 \\
6.13\end{array}$ & 6.13 & 4940 & 15 & 1 & 0.79 \\
\hline & 8-5-F9.1-5\#3-i-2.5-7-6 & $\begin{array}{l}\text { A } \\
B\end{array}$ & 2.0 & $9.1 A_{b}$ & $\begin{array}{l}6.19 \\
6.13 \\
\end{array}$ & 6.16 & 4940 & 15 & 1 & 0.79 \\
\hline & (3@5.5)8-5-F9.1-0-i-2.5-7-6 & $\begin{array}{l}\text { A } \\
B \\
C\end{array}$ & 2.0 & $9.1 A_{b}$ & $\begin{array}{l}6.25 \\
6.13 \\
6.25\end{array}$ & 6.21 & 5160 & 16 & 1 & 0.79 \\
\hline & (3@5.5)8-5-F9.1-5\#3-i-2.5-7-6 & $\begin{array}{l}\text { A } \\
B \\
C\end{array}$ & 2.0 & $9.1 A_{b}$ & $\begin{array}{l}6.13 \\
6.25 \\
6.38\end{array}$ & 6.25 & 5160 & 16 & 1 & 0.79 \\
\hline & (4@3.7)8-5-T9.5-0-i-2.5-6.5-6 & $\begin{array}{l}\text { A } \\
B \\
C \\
D\end{array}$ & 1.4 & $9.5 A_{b}$ & $\begin{array}{l}6.19 \\
6.13 \\
6.19 \\
6.00\end{array}$ & 6.13 & 5160 & 16 & 1 & 0.79 \\
\hline & (4@3.7)8-5-F9.1-5\#3-i-2.5-7-6 & $\begin{array}{l}\text { A } \\
B \\
C \\
D\end{array}$ & 2.0 & $9.1 A_{b}$ & $\begin{array}{l}6.00 \\
6.00 \\
6.00 \\
6.13\end{array}$ & 6.03 & 5160 & 16 & 1 & 0.79 \\
\hline \multirow{11}{*}{ Group 13} & $5-5-F 4.0-0-i-2.5-5-4$ & $\begin{array}{l}A \\
B\end{array}$ & 2.2 & $4.0 A_{b}$ & $\begin{array}{l}4.00 \\
4.13\end{array}$ & 4.06 & 4810 & 8 & 0.625 & 0.31 \\
\hline & 5-5-F13.1-0-i-2.5-5-4 & $\begin{array}{l}A \\
B\end{array}$ & 2.2 & $13.1 A_{b}$ & $\begin{array}{l}4.25 \\
4.56\end{array}$ & 4.41 & 4810 & 8 & 0.625 & 0.31 \\
\hline & 5-5-F4.0-2\#3-i-2.5-5-4 & $\begin{array}{l}A \\
B\end{array}$ & 2.2 & $4.0 A_{b}$ & $\begin{array}{l}3.88 \\
3.75\end{array}$ & 3.81 & 4810 & 8 & 0.625 & 0.31 \\
\hline & 5-5-F13.1-2\#3-i-2.5-5-4 & $\begin{array}{l}A \\
B\end{array}$ & 2.2 & $13.1 A_{b}$ & $\begin{array}{l}4.25 \\
3.94 \\
\end{array}$ & 4.09 & 4810 & 8 & 0.625 & 0.31 \\
\hline & 5-5-F4.0-5\#3-i-2.5-5-4 & $\begin{array}{l}A \\
B\end{array}$ & 2.2 & $4.0 A_{b}$ & $\begin{array}{l}3.94 \\
4.38 \\
\end{array}$ & 4.16 & 4810 & 8 & 0.625 & 0.31 \\
\hline & 5-5-F13.1-5\#3-i-2.5-5-4 & $\begin{array}{l}A \\
B\end{array}$ & 2.2 & $13.1 A_{b}$ & $\begin{array}{l}4.13 \\
4.25 \\
\end{array}$ & 4.19 & 4690 & 7 & 0.625 & 0.31 \\
\hline & 5-5-F4.0-0-i-2.5-3-6 & $\begin{array}{l}\text { A } \\
B\end{array}$ & 2.2 & $4.0 A_{b}$ & $\begin{array}{l}6.00 \\
6.00 \\
\end{array}$ & 6.00 & 4690 & 7 & 0.625 & 0.31 \\
\hline & 5-5-F13.1-0-i-2.5-3-6 & $\begin{array}{l}A \\
B\end{array}$ & 2.2 & $13.1 A_{b}$ & $\begin{array}{l}6.13 \\
6.31 \\
\end{array}$ & 6.22 & 4690 & 7 & 0.625 & 0.31 \\
\hline & 5-5-F4.0-2\#3-i-2.5-3-6 & $\begin{array}{l}\text { A } \\
B\end{array}$ & 2.2 & $4.0 A_{b}$ & $\begin{array}{l}6.00 \\
6.00 \\
\end{array}$ & 6.00 & 4690 & 7 & 0.625 & 0.31 \\
\hline & 5-5-F13.1-2\#3-i-2.5-3-6 & $\begin{array}{l}A \\
B\end{array}$ & 2.2 & $13.1 A_{b}$ & $\begin{array}{l}5.88 \\
6.00 \\
\end{array}$ & 5.94 & 4690 & 7 & 0.625 & 0.31 \\
\hline & 5-5-F4.0-5\#3-i-2.5-3-6 & $\begin{array}{l}\text { A } \\
B\end{array}$ & 2.2 & $4.0 A_{b}$ & $\begin{array}{l}6.00 \\
6.13\end{array}$ & 6.06 & 4690 & 7 & 0.625 & 0.31 \\
\hline
\end{tabular}


Table A.2 Cont. Comprehensive test results and data for beam-column joint specimens

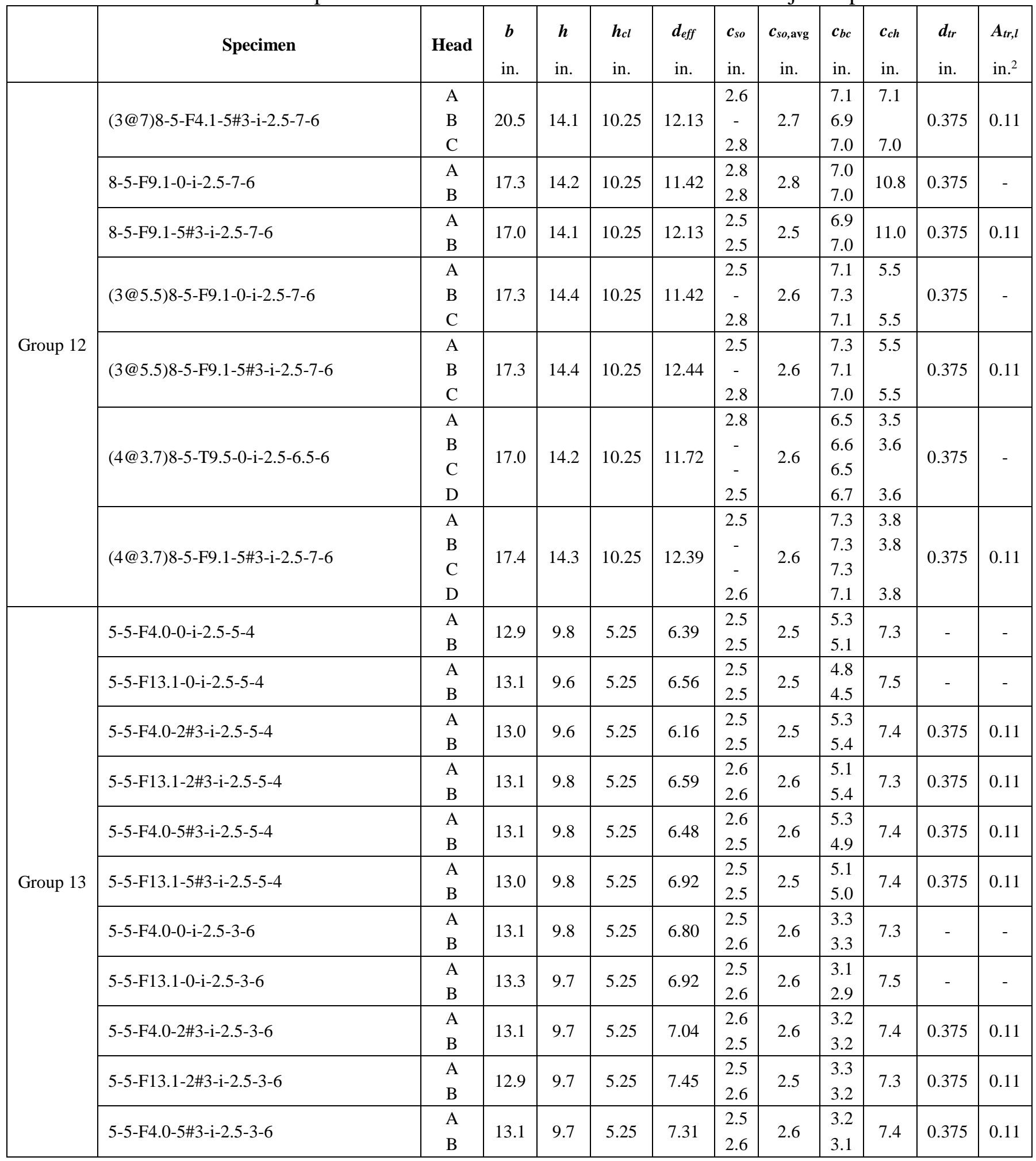


Table A.2 Cont. Comprehensive test results and data for beam-column joint specimens

\begin{tabular}{|c|c|c|c|c|c|c|c|c|c|c|c|}
\hline & Specimen & Head & $N$ & $\begin{array}{l}\boldsymbol{s t r}^{*} \\
\text { in. }\end{array}$ & $\begin{array}{l}\boldsymbol{A}_{t t} \\
\text { in. }^{2}\end{array}$ & $\begin{array}{l}\boldsymbol{d}_{\text {tro }} \\
\text { in. }\end{array}$ & $\begin{array}{l}\text { Stro }^{*} \\
\text { in. }\end{array}$ & $\begin{array}{l}\boldsymbol{A}_{a b} \\
\text { in. }^{2}\end{array}$ & $n$ & $\begin{array}{l}\text { A }_{h s} \\
\text { in. }^{2}\end{array}$ & $\begin{array}{c}\text { Long. } \\
\text { Reinf. } \\
\text { Layout }\end{array}$ \\
\hline \multirow{7}{*}{ Group 12} & (3@7)8-5-F4.1-5\#3-i-2.5-7-6 & $\begin{array}{l}\mathrm{A} \\
\mathrm{B} \\
\mathrm{C}\end{array}$ & 10 & $\begin{array}{c}3 \\
(1.5)\end{array}$ & 0.66 & 0.5 & $\begin{array}{c}4 \\
(2)\end{array}$ & 0.80 & 3 & 2.37 & A8 \\
\hline & 8-5-F9.1-0-i-2.5-7-6 & $\begin{array}{l}\text { A } \\
\text { B }\end{array}$ & - & - & - & 0.375 & $\begin{array}{c}3 \\
(1.5)\end{array}$ & 0.66 & 2 & 1.58 & A4 \\
\hline & 8-5-F9.1-5\#3-i-2.5-7-6 & $\begin{array}{l}\text { A } \\
\text { B }\end{array}$ & 10 & $\begin{array}{c}3 \\
(1.5)\end{array}$ & 0.66 & 0.375 & $\begin{array}{c}3 \\
(1.5)\end{array}$ & 0.66 & 2 & 1.58 & A9 \\
\hline & (3@5.5)8-5-F9.1-0-i-2.5-7-6 & $\begin{array}{l}\mathrm{A} \\
\mathrm{B} \\
\mathrm{C}\end{array}$ & - & - & - & 0.375 & $\begin{array}{c}3 \\
(1.5)\end{array}$ & 0.66 & 3 & 2.37 & A5 \\
\hline & (3@5.5)8-5-F9.1-5\#3-i-2.5-7-6 & $\begin{array}{l}\mathrm{A} \\
\mathrm{B} \\
\mathrm{C}\end{array}$ & 10 & $\begin{array}{c}3 \\
(1.5)\end{array}$ & 0.66 & 0.5 & $\begin{array}{c}4 \\
(2)\end{array}$ & 0.80 & 3 & 2.37 & A5 \\
\hline & (4@3.7)8-5-T9.5-0-i-2.5-6.5-6 & $\begin{array}{l}\text { A } \\
B \\
C \\
D\end{array}$ & - & - & - & 0.375 & $\begin{array}{c}3 \\
(1.5)\end{array}$ & 0.66 & 4 & 3.16 & A5 \\
\hline & (4@3.7)8-5-F9.1-5\#3-i-2.5-7-6 & $\begin{array}{l}\text { A } \\
\text { B } \\
\text { C } \\
\text { D }\end{array}$ & 10 & $\begin{array}{c}3 \\
(1.5)\end{array}$ & 0.66 & 0.5 & $\begin{array}{c}3 \\
(1.5)\end{array}$ & 1.20 & 4 & 3.16 & A5 \\
\hline \multirow{11}{*}{ Group 13} & $5-5-F 4.0-0-i-2.5-5-4$ & $\begin{array}{l}\mathrm{A} \\
\mathrm{B}\end{array}$ & - & - & - & 0.375 & $\begin{array}{c}3.5 \\
(1.75)\end{array}$ & 0.22 & 2 & 0.62 & A4 \\
\hline & 5-5-F13.1-0-i-2.5-5-4 & $\begin{array}{l}\mathrm{A} \\
\mathrm{B}\end{array}$ & - & - & - & 0.375 & $\begin{array}{c}3.5 \\
(1.75) \\
\end{array}$ & 0.22 & 2 & 0.62 & A4 \\
\hline & 5-5-F4.0-2\#3-i-2.5-5-4 & $\begin{array}{l}\text { A } \\
B\end{array}$ & 4 & $\begin{array}{c}3.5 \\
(2.625)\end{array}$ & 0.22 & 0.375 & $\begin{array}{c}3.5 \\
(1.75)\end{array}$ & 0.22 & 2 & 0.62 & A4 \\
\hline & 5-5-F13.1-2\#3-i-2.5-5-4 & $\begin{array}{l}\mathrm{A} \\
\mathrm{B}\end{array}$ & 4 & $\begin{array}{c}3.5 \\
(2.625)\end{array}$ & 0.22 & 0.375 & $\begin{array}{c}3.5 \\
(1.75)\end{array}$ & 0.22 & 2 & 0.62 & A4 \\
\hline & 5-5-F4.0-5\#3-i-2.5-5-4 & $\begin{array}{l}\mathrm{A} \\
\mathrm{B}\end{array}$ & 10 & $\begin{array}{c}1.75 \\
(0.875)\end{array}$ & 0.66 & 0.375 & $\begin{array}{c}3.5 \\
(1.75)\end{array}$ & 0.22 & 2 & 0.62 & A4 \\
\hline & 5-5-F13.1-5\#3-i-2.5-5-4 & $\begin{array}{l}\mathrm{A} \\
\mathrm{B}\end{array}$ & 10 & $\begin{array}{c}1.75 \\
(0.875)\end{array}$ & 0.66 & 0.375 & $\begin{array}{c}3.5 \\
(1.75) \\
\end{array}$ & 0.22 & 2 & 0.62 & A4 \\
\hline & $5-5-F 4.0-0-i-2.5-3-6$ & $\begin{array}{l}\text { A } \\
\text { B }\end{array}$ & - & - & - & 0.375 & $\begin{array}{c}3.5 \\
(1.75) \\
\end{array}$ & 0.22 & 2 & 0.62 & A4 \\
\hline & 5-5-F13.1-0-i-2.5-3-6 & $\begin{array}{l}\mathrm{A} \\
\mathrm{B}\end{array}$ & - & - & - & 0.375 & $\begin{array}{c}3.5 \\
(1.75)\end{array}$ & 0.22 & 2 & 0.62 & A4 \\
\hline & 5-5-F4.0-2\#3-i-2.5-3-6 & $\begin{array}{l}\text { A } \\
\text { B }\end{array}$ & 4 & $\begin{array}{c}3.5 \\
(2.625)\end{array}$ & 0.22 & 0.375 & $\begin{array}{c}3.5 \\
(1.75)\end{array}$ & 0.22 & 2 & 0.62 & A4 \\
\hline & 5-5-F13.1-2\#3-i-2.5-3-6 & $\begin{array}{l}\mathrm{A} \\
\mathrm{B}\end{array}$ & 4 & $\begin{array}{c}3.5 \\
(2.625) \\
\end{array}$ & 0.22 & 0.375 & $\begin{array}{c}3.5 \\
(1.75) \\
\end{array}$ & 0.22 & 2 & 0.62 & A4 \\
\hline & 5-5-F4.0-5\#3-i-2.5-3-6 & $\begin{array}{l}\mathrm{A} \\
\mathrm{B}\end{array}$ & 10 & $\begin{array}{c}1.75 \\
(0.875)\end{array}$ & 0.66 & 0.375 & $\begin{array}{c}3.5 \\
(1.75)\end{array}$ & 0.22 & 2 & 0.62 & A4 \\
\hline
\end{tabular}

${ }^{*}$ Value in parenthesis is the spacing between the first hoop and the center of the headed bar 
Table A.2 Cont. Comprehensive test results and data for beam-column joint specimens

\begin{tabular}{|c|c|c|c|c|c|c|c|c|c|c|}
\hline & Specimen & Head & $\begin{array}{c}\text { Failure } \\
\text { Type }\end{array}$ & $\begin{array}{c}\text { Lead (Head) } \\
\text { Slip } \\
\text { in. }\end{array}$ & $\begin{array}{l}\boldsymbol{T}_{\max } \\
\text { kips }\end{array}$ & $\begin{array}{c}\boldsymbol{f}_{s u, \max } \\
\mathrm{ksi}\end{array}$ & $\begin{array}{l}T_{\text {ind }} \\
\text { kips }\end{array}$ & $\begin{array}{l}\boldsymbol{T}_{\text {total }} \\
\text { kips }\end{array}$ & $\begin{array}{c}T \\
\text { kips }\end{array}$ & $\begin{array}{l}f_{s u} \\
\mathrm{ksi}\end{array}$ \\
\hline \multirow{7}{*}{ Group 12} & (3@7)8-5-F4.1-5\#3-i-2.5-7-6 & $\begin{array}{l}\text { A } \\
\text { B } \\
\text { C }\end{array}$ & CB & $\begin{array}{l}- \\
- \\
-\end{array}$ & $\begin{array}{l}44.1 \\
35.2 \\
47.5\end{array}$ & $\begin{array}{l}55.8 \\
44.6 \\
60.1 \\
\end{array}$ & $\begin{array}{l}44.1 \\
35.2 \\
47.5 \\
\end{array}$ & 126.8 & 42.3 & 53.5 \\
\hline & 8-5-F9.1-0-i-2.5-7-6 & $\begin{array}{l}\text { A } \\
\text { B }\end{array}$ & CB & $\begin{array}{c}0.001 \\
-\end{array}$ & $\begin{array}{l}32.4 \\
34.8 \\
\end{array}$ & $\begin{array}{l}41.0 \\
44.1 \\
\end{array}$ & $\begin{array}{l}32.0 \\
34.8 \\
\end{array}$ & 66.8 & 33.4 & 42.3 \\
\hline & 8-5-F9.1-5\#3-i-2.5-7-6 & $\begin{array}{l}\text { A } \\
\text { B }\end{array}$ & CB & $\begin{array}{l}0.017 \\
0.033\end{array}$ & $\begin{array}{l}53.4 \\
54.3\end{array}$ & $\begin{array}{l}67.6 \\
68.7\end{array}$ & $\begin{array}{l}53.4 \\
54.2\end{array}$ & 107.6 & 53.8 & 68.1 \\
\hline & (3@5.5)8-5-F9.1-0-i-2.5-7-6 & $\begin{array}{l}\text { A } \\
B \\
C\end{array}$ & CB & $\begin{array}{c}0.014 \\
- \\
0.015 \\
\end{array}$ & $\begin{array}{l}28.6 \\
13.9 \\
26.4 \\
\end{array}$ & $\begin{array}{l}36.2 \\
17.6 \\
33.4 \\
\end{array}$ & $\begin{array}{l}28.6 \\
13.9 \\
26.4 \\
\end{array}$ & 68.9 & 23.0 & 29.1 \\
\hline & (3@5.5)8-5-F9.1-5\#3-i-2.5-7-6 & $\begin{array}{l}\text { A } \\
\text { B } \\
\text { C }\end{array}$ & CB & $\begin{array}{c}0.025 \\
- \\
0.022 \\
\end{array}$ & $\begin{array}{l}40.5 \\
46.5 \\
43.0\end{array}$ & $\begin{array}{l}51.3 \\
58.9 \\
54.4 \\
\end{array}$ & $\begin{array}{l}39.9 \\
46.5 \\
43.0 \\
\end{array}$ & 129.4 & 43.1 & 54.6 \\
\hline & (4@3.7)8-5-T9.5-0-i-2.5-6.5-6 & $\begin{array}{l}\text { A } \\
B \\
C \\
D\end{array}$ & CB & $\begin{array}{c}0.001 \\
0.016 \\
- \\
0.024\end{array}$ & $\begin{array}{l}25.9 \\
14.6 \\
17.8 \\
28.8\end{array}$ & $\begin{array}{l}32.8 \\
18.5 \\
22.5 \\
36.5 \\
\end{array}$ & $\begin{array}{l}25.9 \\
14.6 \\
17.8 \\
28.6 \\
\end{array}$ & 86.9 & 21.7 & 27.5 \\
\hline & (4@3.7)8-5-F9.1-5\#3-i-2.5-7-6 & $\begin{array}{l}\text { A } \\
B \\
C \\
D\end{array}$ & CB & $\begin{array}{c}- \\
- \\
- \\
0.023\end{array}$ & $\begin{array}{l}39.5 \\
31.5 \\
20.4 \\
35.1 \\
\end{array}$ & $\begin{array}{l}50.0 \\
39.9 \\
25.8 \\
44.4 \\
\end{array}$ & $\begin{array}{l}39.5 \\
31.5 \\
20.4 \\
35.1 \\
\end{array}$ & 126.5 & 31.6 & 40.0 \\
\hline \multirow{11}{*}{ Group 13} & $5-5-F 4.0-0-i-2.5-5-4$ & $\begin{array}{l}\mathrm{A} \\
\mathrm{B}\end{array}$ & CB & - & $\begin{array}{l}25.9 \\
23.1 \\
\end{array}$ & $\begin{array}{l}83.5 \\
74.5 \\
\end{array}$ & $\begin{array}{l}25.9 \\
23.1 \\
\end{array}$ & 49.1 & 24.5 & 79.0 \\
\hline & 5-5-F13.1-0-i-2.5-5-4 & $\begin{array}{l}\mathrm{A} \\
\mathrm{B}\end{array}$ & CB & - & $\begin{array}{l}26.6 \\
30.2 \\
\end{array}$ & $\begin{array}{l}85.8 \\
97.4 \\
\end{array}$ & $\begin{array}{l}26.5 \\
29.9 \\
\end{array}$ & 56.4 & 28.2 & 91.0 \\
\hline & 5-5-F4.0-2\#3-i-2.5-5-4 & $\begin{array}{l}\mathrm{A} \\
\mathrm{B}\end{array}$ & CB & - & $\begin{array}{l}20.1 \\
19.2 \\
\end{array}$ & $\begin{array}{l}64.8 \\
61.9 \\
\end{array}$ & $\begin{array}{l}20.1 \\
19.2 \\
\end{array}$ & 39.3 & 19.7 & 63.5 \\
\hline & 5-5-F13.1-2\#3-i-2.5-5-4 & $\begin{array}{l}\text { A } \\
\text { B }\end{array}$ & CB & - & $\begin{array}{l}28.6 \\
30.1\end{array}$ & $\begin{array}{l}92.3 \\
97.1\end{array}$ & $\begin{array}{l}27.9 \\
29.8\end{array}$ & 57.7 & 28.9 & 93.2 \\
\hline & 5-5-F4.0-5\#3-i-2.5-5-4 & $\begin{array}{l}\mathrm{A} \\
\mathrm{B}\end{array}$ & CB & - & $\begin{array}{l}27.0 \\
26.1^{\ddagger} \\
\end{array}$ & $\begin{array}{l}87.1 \\
84.2^{\ddagger} \\
\end{array}$ & $\begin{array}{l}27.0 \\
26.0 \\
\end{array}$ & 53.0 & 26.5 & 85.5 \\
\hline & 5-5-F13.1-5\#3-i-2.5-5-4 & $\begin{array}{l}\mathrm{A} \\
\mathrm{B}\end{array}$ & CB & - & $\begin{array}{l}35.5 \\
34.8 \\
\end{array}$ & $\begin{array}{l}114.5 \\
112.3 \\
\end{array}$ & $\begin{array}{l}35.5 \\
34.8 \\
\end{array}$ & 70.4 & 35.2 & 113.5 \\
\hline & 5-5-F4.0-0-i-2.5-3-6 & $\begin{array}{l}\mathrm{A} \\
\mathrm{B}\end{array}$ & SB & - & $\begin{array}{l}34.6^{\ddagger} \\
33.0 \\
\end{array}$ & $\begin{array}{l}111.6^{\ddagger} \\
106.5 \\
\end{array}$ & $\begin{array}{l}32.9 \\
32.6 \\
\end{array}$ & 65.5 & 32.7 & 105.5 \\
\hline & 5-5-F13.1-0-i-2.5-3-6 & $\begin{array}{l}\mathrm{A} \\
\mathrm{B}\end{array}$ & SB/FP & - & $\begin{array}{l}33.2^{\ddagger} \\
37.6\end{array}$ & $\begin{array}{l}107.1^{\ddagger} \\
121.3 \\
\end{array}$ & $\begin{array}{l}33.1 \\
37.5 \\
\end{array}$ & 70.6 & 35.3 & 113.9 \\
\hline & 5-5-F4.0-2\#3-i-2.5-3-6 & $\begin{array}{l}\mathrm{A} \\
\mathrm{B}\end{array}$ & SB/FP & - & $\begin{array}{l}40.0 \\
40.3 \\
\end{array}$ & $\begin{array}{l}129.0 \\
130.0 \\
\end{array}$ & $\begin{array}{l}35.5 \\
40.3 \\
\end{array}$ & 75.7 & 37.9 & 122.3 \\
\hline & 5-5-F13.1-2\#3-i-2.5-3-6 & $\begin{array}{l}\mathrm{A} \\
\mathrm{B}\end{array}$ & SB/FP & - & $\begin{array}{l}46.3^{\ddagger} \\
46.6 \\
\end{array}$ & $\begin{array}{l}149.4^{\ddagger} \\
150.3 \\
\end{array}$ & $\begin{array}{l}46.3 \\
46.5 \\
\end{array}$ & 92.8 & 46.4 & 149.7 \\
\hline & 5-5-F4.0-5\#3-i-2.5-3-6 & $\begin{array}{l}\text { A } \\
B\end{array}$ & SB/FP & - & $\begin{array}{l}42.4 \\
44.6\end{array}$ & $\begin{array}{l}136.8 \\
143.9\end{array}$ & $\begin{array}{l}42.4 \\
44.6\end{array}$ & 86.9 & 43.5 & 140.3 \\
\hline
\end{tabular}

${ }^{\ddagger}$ No anchorage failure on the bar 
Table A.2 Cont. Comprehensive test results and data for beam-column joint specimens

\begin{tabular}{|c|c|c|c|c|c|c|c|c|c|c|}
\hline & Specimen & Head & $\begin{array}{l}\boldsymbol{c}_{\boldsymbol{o}} \\
\text { in. }\end{array}$ & $A_{b r g}$ & $\begin{array}{l}\ell_{e h} \\
\text { in. }\end{array}$ & $\begin{array}{c}\ell \text { eh, avg } \\
\text { in. }\end{array}$ & $\begin{array}{l}\boldsymbol{f}_{\mathrm{cm}} \\
\mathrm{psi}\end{array}$ & $\begin{array}{l}\text { Age } \\
\text { days }\end{array}$ & $\begin{array}{l}d_{b} \\
\text { in. }\end{array}$ & $\begin{array}{c}\boldsymbol{A}_{\boldsymbol{b}} \\
\text { in. }^{2}\end{array}$ \\
\hline \multirow{14}{*}{ Group 14} & 5-12-F4.0-0-i-2.5-5-4 & $\begin{array}{l}\text { A } \\
B\end{array}$ & 2.2 & $4.0 A_{b}$ & $\begin{array}{l}4.13 \\
4.00\end{array}$ & 4.06 & 11030 & 35 & 0.625 & 0.31 \\
\hline & 5-12-F13.1-0-i-2.5-5-4 & $\begin{array}{l}\mathrm{A} \\
\mathrm{B}\end{array}$ & 2.2 & $13.1 A_{b}$ & $\begin{array}{l}4.13 \\
4.13\end{array}$ & 4.13 & 11030 & 35 & 0.625 & 0.31 \\
\hline & 5-12-F4.0-2\#3-i-2.5-5-4 & $\begin{array}{l}\text { A } \\
B\end{array}$ & 2.2 & $4.0 A_{b}$ & $\begin{array}{l}4.13 \\
4.13\end{array}$ & 4.13 & 11030 & 35 & 0.625 & 0.31 \\
\hline & 5-12-F13.1-2\#3-i-2.5-5-4 & $\begin{array}{l}A \\
B\end{array}$ & 2.2 & $13.1 A_{b}$ & $\begin{array}{l}4.06 \\
4.13\end{array}$ & 4.09 & 11030 & 35 & 0.625 & 0.31 \\
\hline & 5-12-F4.0-5\#3-i-2.5-5-4 & $\begin{array}{l}A \\
B\end{array}$ & 2.2 & $4.0 A_{b}$ & $\begin{array}{l}4.19 \\
4.25\end{array}$ & 4.22 & 11030 & 35 & 0.625 & 0.31 \\
\hline & 5-12-F13.1-5\#3-i-2.5-5-4 & $\begin{array}{l}\mathrm{A} \\
\mathrm{B}\end{array}$ & 2.2 & $13.1 A_{b}$ & $\begin{array}{l}4.13 \\
4.13\end{array}$ & 4.13 & 11030 & 35 & 0.625 & 0.31 \\
\hline & 5-12-F4.0-0-i-2.5-3-6 & $\begin{array}{l}A \\
B\end{array}$ & 2.2 & $4.0 A_{b}$ & $\begin{array}{l}6.00 \\
6.00\end{array}$ & 6.00 & 11030 & 36 & 0.625 & 0.31 \\
\hline & 5-12-F13.1-0-i-2.5-3-6 & $\begin{array}{l}A \\
B\end{array}$ & 2.2 & $13.1 A_{b}$ & $\begin{array}{l}6.00 \\
6.06 \\
\end{array}$ & 6.03 & 11030 & 36 & 0.625 & 0.31 \\
\hline & (3@5.9)5-12-F4.0-0-i-2.5-4-5 & $\begin{array}{l}\text { A } \\
\text { B } \\
\text { C }\end{array}$ & 2.2 & $4.0 A_{b}$ & $\begin{array}{l}5.06 \\
5.06 \\
5.00 \\
\end{array}$ & 5.04 & 11030 & 36 & 0.625 & 0.31 \\
\hline & (3@5.9)5-12-F4.0-2\#3-i-2.5-4-5 & $\begin{array}{l}\text { A } \\
B \\
C\end{array}$ & 2.2 & $4.0 A_{b}$ & $\begin{array}{l}5.13 \\
5.13 \\
5.19\end{array}$ & 5.15 & 11030 & 36 & 0.625 & 0.31 \\
\hline & (3@5.9)5-12-F4.0-5\#3-i-2.5-4-5 & $\begin{array}{l}\mathrm{A} \\
\mathrm{B} \\
\mathrm{C}\end{array}$ & 2.2 & $4.0 A_{b}$ & $\begin{array}{l}5.19 \\
4.88 \\
5.00 \\
\end{array}$ & 5.02 & 11030 & 36 & 0.625 & 0.31 \\
\hline & (4@3.9)5-12-F4.0-0-i-2.5-4-5 & $\begin{array}{l}\text { A } \\
B \\
\text { C } \\
\text { D }\end{array}$ & 2.2 & $4.0 A_{b}$ & $\begin{array}{l}5.19 \\
5.13 \\
5.25 \\
5.19\end{array}$ & 5.19 & 11030 & 39 & 0.625 & 0.31 \\
\hline & (4@3.9)5-12-F4.0-2\#3-i-2.5-4-5 & $\begin{array}{l}\text { A } \\
B \\
C \\
D\end{array}$ & 2.2 & $4.0 A_{b}$ & $\begin{array}{l}5.00 \\
5.00 \\
5.13 \\
5.00 \\
\end{array}$ & 5.03 & 11030 & 39 & 0.625 & 0.31 \\
\hline & (4@3.9)5-12-F4.0-5\#3-i-2.5-4-5 & $\begin{array}{l}\text { A } \\
B \\
\text { C } \\
\text { D }\end{array}$ & 2.2 & $4.0 A_{b}$ & $\begin{array}{l}5.25 \\
5.13 \\
5.19 \\
5.19\end{array}$ & 5.19 & 11030 & 39 & 0.625 & 0.31 \\
\hline \multirow{3}{*}{ Group 15} & 11-5a-F3.8-0-i-2.5-3-17 & $\begin{array}{l}\mathrm{A} \\
\mathrm{B}\end{array}$ & 2.0 & $3.8 A_{b}$ & $\begin{array}{l}16.38 \\
16.75 \\
\end{array}$ & 16.56 & 4050 & 36 & 1.41 & 1.56 \\
\hline & 11-5a-F3.8-2\#3-i-2.5-3-17 & $\begin{array}{l}A \\
B\end{array}$ & 2.0 & $3.8 A_{b}$ & $\begin{array}{l}17.44 \\
17.44 \\
\end{array}$ & 17.44 & 4050 & 36 & 1.41 & 1.56 \\
\hline & 11-5a-F3.8-6\#3-i-2.5-3-17 & $\begin{array}{l}A \\
B\end{array}$ & 2.0 & $3.8 A_{b}$ & $\begin{array}{l}16.75 \\
16.69\end{array}$ & 16.72 & 4050 & 36 & 1.41 & 1.56 \\
\hline
\end{tabular}


Table A.2 Cont. Comprehensive test results and data for beam-column joint specimens

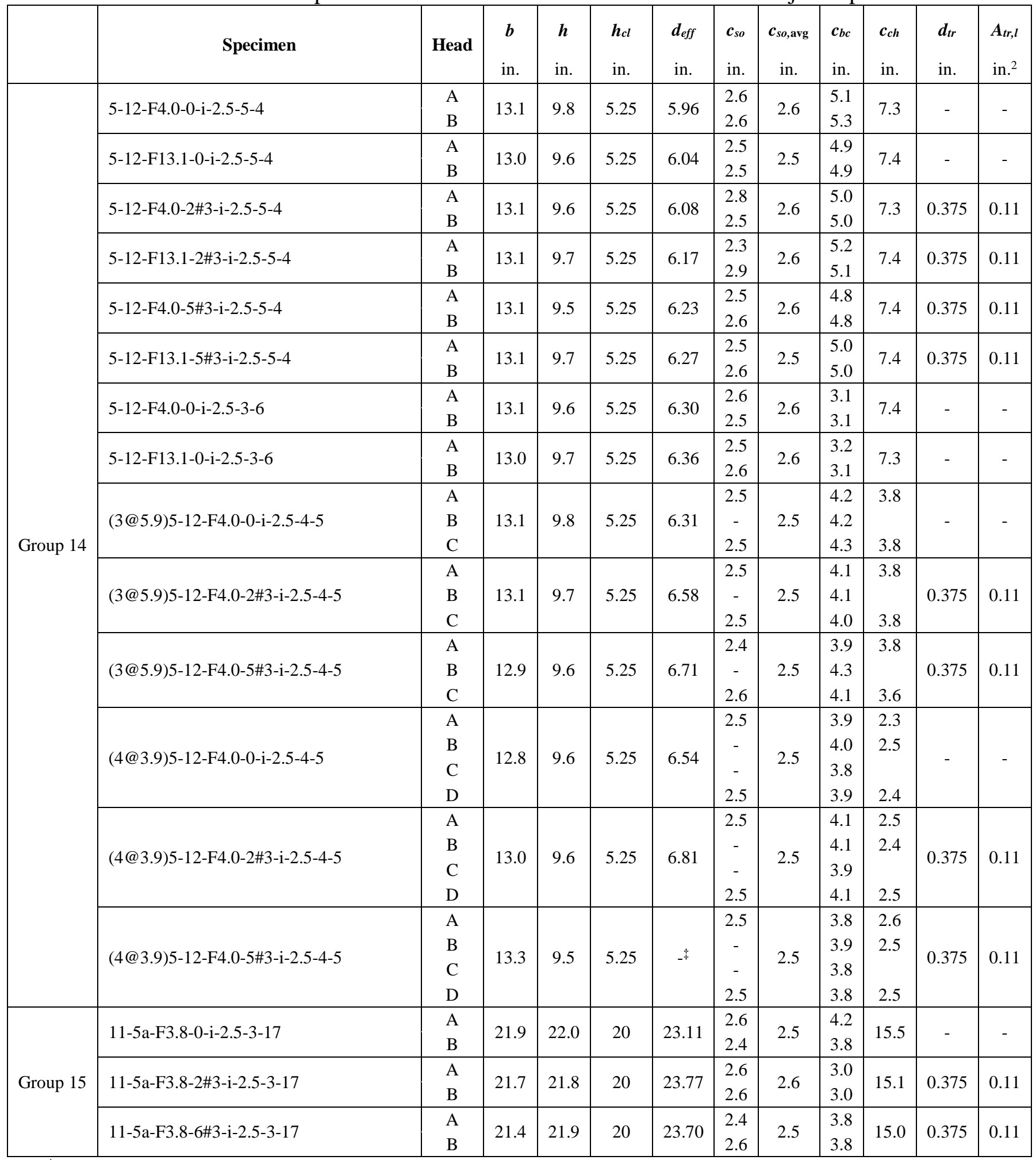

\footnotetext{
${ }^{\ddagger} d_{\text {eff }}$ was not calculated for specimen with bar yielding
} 
Table A.2 Cont. Comprehensive test results and data for beam-column joint specimens

\begin{tabular}{|c|c|c|c|c|c|c|c|c|c|c|c|}
\hline & Specimen & Head & $N$ & $\begin{array}{l}\boldsymbol{s}_{t r}^{*} \\
\text { in. }\end{array}$ & $\begin{array}{l}\boldsymbol{A}_{\boldsymbol{t t}} \\
\text { in. }^{2}\end{array}$ & $\begin{array}{l}\boldsymbol{d}_{\text {tro }} \\
\text { in. }\end{array}$ & $\begin{array}{l}\text { Stro }^{*} \\
\text { in. }\end{array}$ & $\begin{array}{l}A_{a b} \\
\text { in. }^{2}\end{array}$ & $n$ & $\begin{array}{l}A_{h s} \\
\text { in. }^{2}\end{array}$ & $\begin{array}{c}\text { Long. } \\
\text { Reinf. } \\
\text { Layout }\end{array}$ \\
\hline \multirow{14}{*}{ Group 14} & 5-12-F4.0-0-i-2.5-5-4 & $\begin{array}{l}\text { A } \\
B\end{array}$ & - & - & - & 0.375 & $\begin{array}{c}3.5 \\
(1.75)\end{array}$ & 0.22 & 2 & 0.62 & A4 \\
\hline & 5-12-F13.1-0-i-2.5-5-4 & $\begin{array}{l}\mathrm{A} \\
\mathrm{B}\end{array}$ & - & - & - & 0.375 & $\begin{array}{c}3.5 \\
(1.75) \\
\end{array}$ & 0.22 & 2 & 0.62 & A4 \\
\hline & 5-12-F4.0-2\#3-i-2.5-5-4 & $\begin{array}{l}\text { A } \\
\text { B }\end{array}$ & 4 & $\begin{array}{c}3.5 \\
(2.625)\end{array}$ & 0.22 & 0.375 & $\begin{array}{c}3.5 \\
(1.75)\end{array}$ & 0.22 & 2 & 0.62 & A4 \\
\hline & 5-12-F13.1-2\#3-i-2.5-5-4 & $\begin{array}{l}\text { A } \\
B\end{array}$ & 4 & $\begin{array}{c}3.5 \\
(2.625) \\
\end{array}$ & 0.22 & 0.375 & $\begin{array}{c}3.5 \\
(1.75) \\
\end{array}$ & 0.22 & 2 & 0.62 & A4 \\
\hline & 5-12-F4.0-5\#3-i-2.5-5-4 & $\begin{array}{l}\text { A } \\
\text { B }\end{array}$ & 10 & $\begin{array}{c}1.75 \\
(0.875) \\
\end{array}$ & 0.66 & 0.375 & $\begin{array}{c}3.5 \\
(1.75) \\
\end{array}$ & 0.22 & 2 & 0.62 & A4 \\
\hline & 5-12-F13.1-5\#3-i-2.5-5-4 & $\begin{array}{l}\mathrm{A} \\
\mathrm{B}\end{array}$ & 10 & $\begin{array}{c}1.75 \\
(0.875) \\
\end{array}$ & 0.66 & 0.375 & $\begin{array}{c}3.5 \\
(1.75) \\
\end{array}$ & 0.22 & 2 & 0.62 & A4 \\
\hline & 5-12-F4.0-0-i-2.5-3-6 & $\begin{array}{l}\text { A } \\
\text { B }\end{array}$ & - & - & - & 0.375 & $\begin{array}{c}3.5 \\
(1.75)\end{array}$ & 0.22 & 2 & 0.62 & A4 \\
\hline & 5-12-F13.1-0-i-2.5-3-6 & $\begin{array}{l}\text { A } \\
\text { B }\end{array}$ & - & - & - & 0.375 & $\begin{array}{c}3.5 \\
(1.75)\end{array}$ & 0.22 & 2 & 0.62 & A4 \\
\hline & (3@5.9)5-12-F4.0-0-i-2.5-4-5 & $\begin{array}{l}\mathrm{A} \\
\mathrm{B} \\
\mathrm{C}\end{array}$ & - & - & - & 0.375 & $\begin{array}{c}3.5 \\
(1.75)\end{array}$ & 0.22 & 3 & 0.93 & A5 \\
\hline & (3@5.9)5-12-F4.0-2\#3-i-2.5-4-5 & $\begin{array}{l}\text { A } \\
\text { B } \\
\text { C }\end{array}$ & 4 & $\begin{array}{c}3.5 \\
(2.625)\end{array}$ & 0.22 & 0.375 & $\begin{array}{c}3.5 \\
(1.75)\end{array}$ & 0.22 & 3 & 0.93 & A5 \\
\hline & (3@5.9)5-12-F4.0-5\#3-i-2.5-4-5 & $\begin{array}{l}\mathrm{A} \\
\mathrm{B} \\
\mathrm{C} \\
\end{array}$ & 10 & $\begin{array}{c}1.75 \\
(0.875)\end{array}$ & 0.66 & 0.375 & $\begin{array}{c}3.5 \\
(1.75)\end{array}$ & 0.22 & 3 & 0.93 & A5 \\
\hline & (4@3.9)5-12-F4.0-0-i-2.5-4-5 & $\begin{array}{l}\mathrm{A} \\
\mathrm{B} \\
\mathrm{C} \\
\mathrm{D}\end{array}$ & - & - & - & 0.375 & $\begin{array}{c}3.5 \\
(1.75)\end{array}$ & 0.22 & 4 & 1.24 & A5 \\
\hline & (4@3.9)5-12-F4.0-2\#3-i-2.5-4-5 & $\begin{array}{l}\text { A } \\
\text { B } \\
\text { C } \\
\text { D }\end{array}$ & 4 & $\begin{array}{c}3.5 \\
(2.625)\end{array}$ & 0.22 & 0.375 & $\begin{array}{c}3.5 \\
(1.75)\end{array}$ & 0.22 & 4 & 1.24 & A5 \\
\hline & (4@3.9)5-12-F4.0-5\#3-i-2.5-4-5 & $\begin{array}{l}\mathrm{A} \\
\mathrm{B} \\
\mathrm{C} \\
\mathrm{D}\end{array}$ & 10 & $\begin{array}{c}1.75 \\
(0.875)\end{array}$ & 0.22 & 0.375 & $\begin{array}{c}3.5 \\
(1.75)\end{array}$ & 0.18 & 4 & 1.24 & A5 \\
\hline \multirow{3}{*}{ Group 15} & 11-5a-F3.8-0-i-2.5-3-17 & $\begin{array}{l}\mathrm{A} \\
\mathrm{B}\end{array}$ & - & - & - & 0.5 & $\begin{array}{c}4 \\
(2) \\
\end{array}$ & 1.20 & 2 & 3.12 & A10 \\
\hline & 11-5a-F3.8-2\#3-i-2.5-3-17 & $\begin{array}{l}\text { A } \\
\text { B }\end{array}$ & 4 & $\begin{array}{c}8 \\
(6) \\
\end{array}$ & 0.22 & 0.5 & $\begin{array}{c}4 \\
(2) \\
\end{array}$ & 1.20 & 2 & 3.12 & A10 \\
\hline & 11-5a-F3.8-6\#3-i-2.5-3-17 & $\begin{array}{l}\mathrm{A} \\
\mathrm{B}\end{array}$ & 12 & $\begin{array}{c}4 \\
(2) \\
\end{array}$ & 0.66 & 0.5 & $\begin{array}{c}4 \\
(2) \\
\end{array}$ & 1.20 & 2 & 3.12 & A10 \\
\hline
\end{tabular}

${ }^{*}$ Value in parenthesis is the spacing between the first hoop and the center of the headed bar 
Table A.2 Cont. Comprehensive test results and data for beam-column joint specimens

\begin{tabular}{|c|c|c|c|c|c|c|c|c|c|c|}
\hline & Specimen & Head & $\begin{array}{c}\text { Failure } \\
\text { Type }\end{array}$ & $\begin{array}{c}\text { Lead (Head) } \\
\text { Slip } \\
\text { in. }\end{array}$ & $\begin{array}{l}\boldsymbol{T}_{\max } \\
\text { kips }\end{array}$ & $\begin{array}{c}\boldsymbol{f}_{\text {su,max }} \\
\mathrm{ksi}\end{array}$ & $\begin{array}{l}\boldsymbol{T}_{\text {ind }} \\
\text { kips }\end{array}$ & $\begin{array}{l}\boldsymbol{T}_{\text {total }} \\
\text { kips }\end{array}$ & $\begin{array}{c}\boldsymbol{T} \\
\text { kips }\end{array}$ & $\begin{array}{l}f_{s u} \\
\mathrm{ksi}\end{array}$ \\
\hline \multirow{14}{*}{ Group 14} & 5-12-F4.0-0-i-2.5-5-4 & $\begin{array}{l}\text { A } \\
\text { B }\end{array}$ & CB & $\begin{array}{l}0.123 \\
0.055\end{array}$ & $\begin{array}{l}28.9 \\
27.7\end{array}$ & $\begin{array}{l}93.2 \\
89.4\end{array}$ & $\begin{array}{l}28.9 \\
27.7\end{array}$ & 56.6 & 28.3 & 91.3 \\
\hline & 5-12-F13.1-0-i-2.5-5-4 & $\begin{array}{l}\text { A } \\
\text { B }\end{array}$ & $\mathrm{CB}$ & $\begin{array}{l}0.179 \\
0.116\end{array}$ & $\begin{array}{l}32.6 \\
30.2\end{array}$ & $\begin{array}{c}105.2 \\
97.4\end{array}$ & $\begin{array}{l}32.5 \\
30.2\end{array}$ & 62.7 & 31.4 & 101.3 \\
\hline & 5-12-F4.0-2\#3-i-2.5-5-4 & $\begin{array}{l}\text { A } \\
\text { B }\end{array}$ & CB & $\begin{array}{l}0.072 \\
0.015\end{array}$ & $\begin{array}{l}33.7 \\
31.7\end{array}$ & $\begin{array}{l}108.7 \\
102.3\end{array}$ & $\begin{array}{l}33.7 \\
31.7\end{array}$ & 65.4 & 32.7 & 105.5 \\
\hline & 5-12-F13.1-2\#3-i-2.5-5-4 & $\begin{array}{l}\text { A } \\
B\end{array}$ & CB & $\begin{array}{l}0.136 \\
0.025\end{array}$ & $\begin{array}{l}34.4 \\
38.2\end{array}$ & $\begin{array}{l}111.0 \\
123.2\end{array}$ & $\begin{array}{l}34.4 \\
38.2\end{array}$ & 72.5 & 36.3 & 117.1 \\
\hline & 5-12-F4.0-5\#3-i-2.5-5-4 & $\begin{array}{l}\mathrm{A} \\
\mathrm{B}\end{array}$ & CB & $\begin{array}{l}0.196 \\
0.308 \\
\end{array}$ & $\begin{array}{l}40.2 \\
37.5 \\
\end{array}$ & $\begin{array}{l}129.7 \\
121.0 \\
\end{array}$ & $\begin{array}{l}40.2 \\
37.5 \\
\end{array}$ & 77.7 & 38.9 & 125.5 \\
\hline & 5-12-F13.1-5\#3-i-2.5-5-4 & $\begin{array}{l}\text { A } \\
\text { B }\end{array}$ & $\mathrm{CB}$ & $\begin{array}{l}0.172 \\
0.269\end{array}$ & $\begin{array}{l}40.8 \\
39.8\end{array}$ & $\begin{array}{l}131.6 \\
128.4\end{array}$ & $\begin{array}{l}40.8 \\
39.8\end{array}$ & 80.6 & 40.3 & 130.0 \\
\hline & 5-12-F4.0-0-i-2.5-3-6 & $\begin{array}{l}\mathrm{A} \\
\mathrm{B}\end{array}$ & SB & $\begin{array}{l}0.136 \\
0.226 \\
\end{array}$ & $\begin{array}{l}43.9 \\
41.6 \\
\end{array}$ & $\begin{array}{l}141.6 \\
134.2 \\
\end{array}$ & $\begin{array}{l}41.8 \\
41.6 \\
\end{array}$ & 83.5 & 41.7 & 134.5 \\
\hline & 5-12-F13.1-0-i-2.5-3-6 & $\begin{array}{l}\text { A } \\
B\end{array}$ & CB & $\begin{array}{l}0.081 \\
0.327\end{array}$ & $\begin{array}{l}44.7 \\
43.8\end{array}$ & $\begin{array}{l}144.2 \\
141.3\end{array}$ & $\begin{array}{l}44.5 \\
43.8\end{array}$ & 88.3 & 44.2 & 142.6 \\
\hline & (3@5.9)5-12-F4.0-0-i-2.5-4-5 & $\begin{array}{l}\text { A } \\
\text { B } \\
\text { C }\end{array}$ & CB & $\begin{array}{c}- \\
0.100 \\
-\end{array}$ & $\begin{array}{l}27.1 \\
28.9 \\
28.2\end{array}$ & $\begin{array}{l}87.4 \\
93.2 \\
91.0\end{array}$ & $\begin{array}{l}27.0 \\
28.8 \\
28.2\end{array}$ & 84.1 & 28.0 & 90.4 \\
\hline & (3@5.9)5-12-F4.0-2\#3-i-2.5-4-5 & $\begin{array}{l}\text { A } \\
\text { B } \\
\text { C }\end{array}$ & CB & $\begin{array}{c}0.169 \\
- \\
- \\
\end{array}$ & $\begin{array}{l}34.5 \\
35.3 \\
35.6 \\
\end{array}$ & $\begin{array}{l}111.3 \\
113.9 \\
114.8 \\
\end{array}$ & $\begin{array}{l}34.5 \\
35.3 \\
35.6\end{array}$ & 105.4 & 35.1 & 113.3 \\
\hline & (3@5.9)5-12-F4.0-5\#3-i-2.5-4-5 & $\begin{array}{l}\text { A } \\
\text { B } \\
\text { C }\end{array}$ & CB & $\begin{array}{c}0.266 \\
0.216 \\
- \\
\end{array}$ & $\begin{array}{l}42.5 \\
33.3 \\
41.3 \\
\end{array}$ & $\begin{array}{l}137.1 \\
107.4 \\
133.2 \\
\end{array}$ & $\begin{array}{l}42.3 \\
32.7 \\
40.9 \\
\end{array}$ & 115.9 & 38.6 & 124.6 \\
\hline & (4@3.9)5-12-F4.0-0-i-2.5-4-5 & $\begin{array}{l}\text { A } \\
\text { B } \\
\text { C } \\
\text { D }\end{array}$ & CB & $\begin{array}{c}0.099 \\
- \\
0.109 \\
- \\
\end{array}$ & $\begin{array}{c}28.3 \\
-\dagger \\
24.5 \\
24.1\end{array}$ & $\begin{array}{c}91.3 \\
-^{\dagger} \\
79.0 \\
77.7\end{array}$ & $\begin{array}{c}28.3 \\
- \\
24.5 \\
24.1\end{array}$ & $-\dagger$ & $25.6^{\dagger}$ & 82.7 \\
\hline & (4@3.9)5-12-F4.0-2\#3-i-2.5-4-5 & $\begin{array}{l}\text { A } \\
B \\
C \\
D\end{array}$ & CB & $\begin{array}{c}0.123 \\
- \\
0.228 \\
-\end{array}$ & $\begin{array}{c}33.5 \\
-^{\dagger} \\
30.7 \\
28.7\end{array}$ & $\begin{array}{c}108.1 \\
-^{\dagger} \\
99.0 \\
92.6\end{array}$ & $\begin{array}{c}33.3 \\
- \\
30.7 \\
28.7\end{array}$ & $-{ }^{\dagger}$ & $30.9^{\dagger}$ & 99.7 \\
\hline & (4@3.9)5-12-F4.0-5\#3-i-2.5-4-5 & $\begin{array}{l}\text { A } \\
\text { B } \\
\text { C } \\
\text { D }\end{array}$ & $\mathrm{Y}$ & $\begin{array}{l}- \\
- \\
- \\
-\end{array}$ & $\begin{array}{c}48.9 \\
-^{\dagger} \\
51.3 \\
46.9\end{array}$ & $\begin{array}{c}157.7 \\
-^{\dagger} \\
165.5 \\
151.3 \\
\end{array}$ & $\begin{array}{c}48.0 \\
- \\
49.4 \\
46.8 \\
\end{array}$ & $-\dagger$ & $48.1^{\dagger}$ & 155.2 \\
\hline \multirow{3}{*}{ Group 15} & 11-5a-F3.8-0-i-2.5-3-17 & $\begin{array}{l}\mathrm{A} \\
\mathrm{B}\end{array}$ & $\mathrm{CB} / \mathrm{FP}$ & $\begin{array}{l}0.106 \\
0.043 \\
\end{array}$ & $\begin{array}{l}97.1 \\
98.0 \\
\end{array}$ & $\begin{array}{l}62.2 \\
62.8 \\
\end{array}$ & $\begin{array}{l}97.1 \\
98.0 \\
\end{array}$ & 195.1 & 97.5 & 62.5 \\
\hline & 11-5a-F3.8-2\#3-i-2.5-3-17 & $\begin{array}{l}\text { A } \\
\text { B }\end{array}$ & $\mathrm{SB} / \mathrm{FP}$ & $\begin{array}{l}0.337 \\
0.235\end{array}$ & $\begin{array}{l}117.7 \\
133.4\end{array}$ & $\begin{array}{l}75.4 \\
85.5\end{array}$ & $\begin{array}{l}117.7 \\
118.8\end{array}$ & 236.5 & 118.2 & 75.8 \\
\hline & 11-5a-F3.8-6\#3-i-2.5-3-17 & $\begin{array}{l}\text { A } \\
\text { B }\end{array}$ & $\mathrm{SB} / \mathrm{FP}$ & $\begin{array}{l}0.130 \\
0.041\end{array}$ & $\begin{array}{l}119.9 \\
118.0\end{array}$ & $\begin{array}{l}76.9 \\
75.6\end{array}$ & $\begin{array}{l}114.5 \\
118.0\end{array}$ & 232.4 & 116.2 & 74.5 \\
\hline
\end{tabular}

\footnotetext{
${ }^{\dagger}$ Load on headed bar B was not recorded due to a malfunction of load cell; $T$ taken as the average load of the other three bars.
} 
Table A.2 Cont. Comprehensive test results and data for beam-column joint specimens

\begin{tabular}{|c|c|c|c|c|c|c|c|c|c|c|}
\hline & Specimen & Head & $\begin{array}{l}c_{o} \\
\text { in. }\end{array}$ & $A_{b r g}$ & $\begin{array}{l}\ell_{e h} \\
\text { in. }\end{array}$ & $\begin{array}{c}\ell_{e h, a v g} \\
\text { in. }\end{array}$ & $\begin{array}{l}\boldsymbol{f}_{\boldsymbol{c m}} \\
\mathrm{psi}\end{array}$ & $\begin{array}{l}\text { Age } \\
\text { days }\end{array}$ & $\begin{array}{l}\boldsymbol{d}_{\boldsymbol{b}} \\
\text { in. }\end{array}$ & $\begin{array}{l}\boldsymbol{A}_{\boldsymbol{b}} \\
\text { in. }^{2}\end{array}$ \\
\hline \multirow{5}{*}{ Group 15} & 11-5a-F3.8-0-i-2.5-3-12 & $\begin{array}{l}\text { A } \\
\text { B }\end{array}$ & 2.0 & $3.8 A_{b}$ & $\begin{array}{l}12.19 \\
11.81\end{array}$ & 12.00 & 3960 & 35 & 1.41 & 1.56 \\
\hline & 11-5a-F3.8-2\#3-i-2.5-3-12 & $\begin{array}{l}\text { A } \\
\text { B }\end{array}$ & 2.0 & $3.8 A_{b}$ & $\begin{array}{l}11.81 \\
12.19\end{array}$ & 12.00 & 3960 & 35 & 1.41 & 1.56 \\
\hline & 11-5a-F3.8-6\#3-i-2.5-3-12 & $\begin{array}{l}\text { A } \\
\text { B }\end{array}$ & 2.0 & $3.8 A_{b}$ & $\begin{array}{l}12.13 \\
12.06\end{array}$ & 12.09 & 3960 & 35 & 1.41 & 1.56 \\
\hline & 11-5a-F8.6-0-i-2.5-3-12 & $\begin{array}{l}\text { A } \\
\text { B }\end{array}$ & 2.0 & $8.6 A_{b}$ & $\begin{array}{l}12.13 \\
12.13\end{array}$ & 12.13 & 3960 & 35 & 1.41 & 1.56 \\
\hline & 11-5a- F8.6-6\#3-i-2.5-3-12 & $\begin{array}{l}\text { A } \\
\text { B }\end{array}$ & 2.0 & $8.6 A_{b}$ & $\begin{array}{l}12.69 \\
12.44\end{array}$ & 12.56 & 4050 & 36 & 1.41 & 1.56 \\
\hline \multirow{9}{*}{ Group 16} & 8-8-F4.1-0-i-2.5-3-10-DB & $\begin{array}{l}\text { A } \\
\text { B }\end{array}$ & 2.0 & $4.1 A_{b}$ & $\begin{array}{l}9.88 \\
9.88\end{array}$ & 9.88 & 7410 & 49 & 1 & 0.79 \\
\hline & 8-8-F9.1-0-i-2.5-3-10-DB & $\begin{array}{l}\text { A } \\
\text { B }\end{array}$ & 2.0 & $9.1 A_{b}$ & $\begin{array}{l}9.88 \\
9.75 \\
\end{array}$ & 9.81 & 7410 & 49 & 1 & 0.79 \\
\hline & 8-8-F9.1-5\#3-i-2.5-3-10-DB & $\begin{array}{l}\text { A } \\
\text { B }\end{array}$ & 2.0 & $9.1 A_{b}$ & $\begin{array}{l}9.63 \\
9.63\end{array}$ & 9.63 & 7410 & 49 & 1 & 0.79 \\
\hline & 8-5-F4.1-0-i-2.5-3-10-DB & $\begin{array}{l}\text { A } \\
\text { B }\end{array}$ & 2.0 & $4.1 A_{b}$ & $\begin{array}{l}9.88 \\
9.88\end{array}$ & 9.88 & 4880 & 19 & 1 & 0.79 \\
\hline & 8-5-F9.1-0-i-2.5-3-10-DB & $\begin{array}{l}\text { A } \\
\text { B }\end{array}$ & 2.0 & $9.1 A_{b}$ & $\begin{array}{l}9.63 \\
9.88\end{array}$ & 9.75 & 4880 & 19 & 1 & 0.79 \\
\hline & 8-5-F4.1-3\#4-i-2.5-3-10-DB & $\begin{array}{l}\text { A } \\
\text { B }\end{array}$ & 2.0 & $4.1 A_{b}$ & $\begin{array}{l}10.00 \\
10.25\end{array}$ & 10.13 & 4880 & 19 & 1 & 0.79 \\
\hline & 8-5-F9.1-3\#4-i-2.5-3-10-DB & $\begin{array}{l}A \\
B\end{array}$ & 2.0 & $9.1 A_{b}$ & $\begin{array}{l}9.75 \\
9.75\end{array}$ & 9.75 & 4880 & 20 & 1 & 0.79 \\
\hline & 8-5-F4.1-5\#3-i-2.5-3-10-DB & $\begin{array}{l}\text { A } \\
\text { B }\end{array}$ & 2.0 & $4.1 A_{b}$ & $\begin{array}{l}10.25 \\
10.13\end{array}$ & 10.19 & 4880 & 20 & 1 & 0.79 \\
\hline & 8-5-F9.1-5\#3-i-2.5-3-10-DB & $\begin{array}{l}\text { A } \\
\text { B }\end{array}$ & 2.0 & $9.1 A_{b}$ & $\begin{array}{c}10.00 \\
9.88\end{array}$ & 9.94 & 4880 & 20 & 1 & 0.79 \\
\hline \multirow{6}{*}{ Group 17} & 11-8-F3.8-0-i-2.5-3-14.5 & $\begin{array}{l}\text { A } \\
\text { B }\end{array}$ & 2.0 & $3.8 A_{b}$ & $\begin{array}{l}14.50 \\
14.50\end{array}$ & 14.50 & 8660 & 19 & 1.41 & 1.56 \\
\hline & 11-8-F3.8-2\#3-i-2.5-3-14.5 & $\begin{array}{l}\text { A } \\
\text { B }\end{array}$ & 2.0 & $3.8 A_{b}$ & $\begin{array}{l}14.63 \\
14.75\end{array}$ & 14.69 & 8660 & 19 & 1.41 & 1.56 \\
\hline & 11-8-F3.8-6\#3-i-2.5-3-14.5 & $\begin{array}{l}\text { A } \\
\text { B }\end{array}$ & 2.0 & $3.8 A_{b}$ & $\begin{array}{l}14.88 \\
14.50\end{array}$ & 14.69 & 8660 & 19 & 1.41 & 1.56 \\
\hline & (3@5.35)11-8-F3.8-0-i-2.5-3-14.5 & $\begin{array}{l}\text { A } \\
\text { B } \\
\text { C } \\
\end{array}$ & 2.0 & $3.8 A_{b}$ & $\begin{array}{l}14.38 \\
14.75 \\
14.75\end{array}$ & 14.63 & 8720 & 20 & 1.41 & 1.56 \\
\hline & (3@5.35)11-8-F3.8-2\#3-i-2.5-3-14.5 & $\begin{array}{l}\text { A } \\
\text { B } \\
\text { C }\end{array}$ & 2.0 & $3.8 A_{b}$ & $\begin{array}{l}14.50 \\
14.63 \\
14.50\end{array}$ & 14.54 & 8720 & 20 & 1.41 & 1.56 \\
\hline & (3@5.35)11-8-F3.8-6\#3-i-2.5-3-14.5 & $\begin{array}{l}\text { A } \\
\text { B } \\
\text { C }\end{array}$ & 2.0 & $3.8 A_{b}$ & $\begin{array}{l}15.13 \\
14.88 \\
14.75\end{array}$ & 14.92 & 8720 & 20 & 1.41 & 1.56 \\
\hline
\end{tabular}


Table A.2 Cont. Comprehensive test results and data for beam-column joint specimens

\begin{tabular}{|c|c|c|c|c|c|c|c|c|c|c|c|c|}
\hline & Specimen & Head & $\begin{array}{c}\boldsymbol{b} \\
\text { in. }\end{array}$ & $\begin{array}{c}\boldsymbol{h} \\
\text { in. } \\
\end{array}$ & $\begin{array}{l}\boldsymbol{h}_{\boldsymbol{c l}} \\
\text { in. }\end{array}$ & $\begin{array}{l}\boldsymbol{d}_{\text {eff }} \\
\text { in. }\end{array}$ & $\begin{array}{l}c_{s o} \\
\text { in. }\end{array}$ & $\begin{array}{c}\boldsymbol{c}_{\boldsymbol{s o}, \text { avg }} \\
\text { in. }\end{array}$ & $\begin{array}{l}c_{b c} \\
\text { in. }\end{array}$ & $\begin{array}{l}c_{c h} \\
\text { in. }\end{array}$ & $\begin{array}{l}\boldsymbol{d}_{t r} \\
\text { in. }\end{array}$ & $\begin{array}{l}A_{t r, l} \\
\text { in. }^{2}\end{array}$ \\
\hline \multirow{5}{*}{ Group 15} & 11-5a-F3.8-0-i-2.5-3-12 & $\begin{array}{l}\text { A } \\
\text { B } \\
\end{array}$ & 21.7 & 16.5 & 20 & 21.84 & $\begin{array}{l}2.6 \\
2.8\end{array}$ & 2.7 & $\begin{array}{l}2.9 \\
3.3 \\
\end{array}$ & 14.9 & - & - \\
\hline & 11-5a-F3.8-2\#3-i-2.5-3-12 & $\begin{array}{l}\text { A } \\
\text { B } \\
\end{array}$ & 21.4 & 17.1 & 20 & 22.18 & $\begin{array}{l}2.4 \\
2.4\end{array}$ & 2.4 & $\begin{array}{l}3.9 \\
3.5 \\
\end{array}$ & 15.3 & 0.375 & 0.11 \\
\hline & 11-5a-F3.8-6\#3-i-2.5-3-12 & $\begin{array}{l}\text { A } \\
\text { B }\end{array}$ & 21.6 & 17.0 & 20 & 22.53 & $\begin{array}{l}2.8 \\
2.5\end{array}$ & 2.7 & $\begin{array}{l}3.5 \\
3.6\end{array}$ & 14.9 & 0.375 & 0.11 \\
\hline & 11-5a- F8.6-0-i-2.5-3-12 & $\begin{array}{l}\text { A } \\
\text { B }\end{array}$ & 21.7 & 16.8 & 20 & 22.07 & $\begin{array}{l}2.8 \\
2.5\end{array}$ & 2.6 & $\begin{array}{l}3.3 \\
3.3 \\
\end{array}$ & 15.0 & - & - \\
\hline & 11-5a- F8.6-6\#3-i-2.5-3-12 & $\begin{array}{l}\mathrm{A} \\
\mathrm{B} \\
\end{array}$ & 22.0 & 17.3 & 20 & 22.53 & $\begin{array}{l}2.8 \\
2.7\end{array}$ & 2.7 & $\begin{array}{l}3.3 \\
3.5 \\
\end{array}$ & 15.2 & 0.375 & 0.11 \\
\hline \multirow{9}{*}{ Group 16} & 8-8-F4.1-0-i-2.5-3-10-DB & $\begin{array}{l}\text { A } \\
\text { B }\end{array}$ & 17.1 & 14.3 & 20 & 21.38 & $\begin{array}{l}2.5 \\
2.6\end{array}$ & 2.6 & $\begin{array}{l}3.4 \\
3.4\end{array}$ & 11.0 & - & - \\
\hline & 8-8-F9.1-0-i-2.5-3-10-DB & $\begin{array}{l}\text { A } \\
\text { B }\end{array}$ & 17.3 & 14.2 & 20 & 21.42 & $\begin{array}{l}2.6 \\
2.6\end{array}$ & 2.6 & $\begin{array}{l}3.3 \\
3.4\end{array}$ & 11.0 & - & - \\
\hline & 8-8-F9.1-5\#3-i-2.5-3-10-DB & $\begin{array}{l}\text { A } \\
\text { B } \\
\end{array}$ & 17.4 & 14.2 & 20 & 21.88 & $\begin{array}{l}2.5 \\
2.8 \\
\end{array}$ & 2.6 & $\begin{array}{l}3.6 \\
3.6 \\
\end{array}$ & 11.1 & 0.375 & 0.11 \\
\hline & 8-5-F4.1-0-i-2.5-3-10-DB & $\begin{array}{l}\mathrm{A} \\
\mathrm{B} \\
\end{array}$ & 17.4 & 14.1 & 20 & 21.43 & $\begin{array}{l}2.5 \\
2.6\end{array}$ & 2.6 & $\begin{array}{l}3.3 \\
3.3 \\
\end{array}$ & 11.3 & - & - \\
\hline & 8-5-F9.1-0-i-2.5-3-10-DB & $\begin{array}{l}\text { A } \\
\text { B } \\
\end{array}$ & 17.5 & 14.3 & 20 & 21.56 & $\begin{array}{l}2.6 \\
2.6\end{array}$ & 2.6 & $\begin{array}{l}3.6 \\
3.4 \\
\end{array}$ & 11.3 & - & - \\
\hline & 8-5-F4.1-3\#4-i-2.5-3-10-DB & $\begin{array}{l}\text { A } \\
\text { B }\end{array}$ & 17.3 & 14.1 & 20 & 22.27 & $\begin{array}{l}2.5 \\
2.5\end{array}$ & 2.5 & $\begin{array}{l}3.1 \\
2.9\end{array}$ & 11.3 & 0.5 & 0.2 \\
\hline & 8-5-F9.1-3\#4-i-2.5-3-10-DB & $\begin{array}{l}\text { A } \\
\text { B }\end{array}$ & 17.3 & 14.4 & 20 & 22.31 & $\begin{array}{l}2.6 \\
2.6\end{array}$ & 2.6 & $\begin{array}{l}3.6 \\
3.6\end{array}$ & 11.0 & 0.5 & 0.2 \\
\hline & 8-5-F4.1-5\#3-i-2.5-3-10-DB & $\begin{array}{l}\mathrm{A} \\
\mathrm{B} \\
\end{array}$ & 17.5 & 14.3 & 20 & 22.47 & $\begin{array}{l}2.6 \\
2.8 \\
\end{array}$ & 2.7 & $\begin{array}{l}3.1 \\
3.2 \\
\end{array}$ & 11.1 & 0.375 & 0.11 \\
\hline & 8-5-F9.1-5\#3-i-2.5-3-10-DB & $\begin{array}{l}\text { A } \\
\text { B }\end{array}$ & 17.3 & 14.2 & 20 & 22.48 & $\begin{array}{l}2.6 \\
2.6\end{array}$ & 2.6 & $\begin{array}{l}3.2 \\
3.3\end{array}$ & 11.0 & 0.375 & 0.11 \\
\hline \multirow{6}{*}{ Group 17} & 11-8-F3.8-0-i-2.5-3-14.5 & $\begin{array}{l}\text { A } \\
\text { B }\end{array}$ & 21.8 & 19.3 & 20 & 21.54 & $\begin{array}{l}2.8 \\
2.5\end{array}$ & 2.6 & $\begin{array}{l}3.4 \\
3.4\end{array}$ & 15.1 & - & - \\
\hline & 11-8-F3.8-2\#3-i-2.5-3-14.5 & $\begin{array}{l}\text { A } \\
\text { B }\end{array}$ & 21.8 & 19.3 & 20 & 21.72 & $\begin{array}{l}2.5 \\
2.6\end{array}$ & 2.6 & $\begin{array}{l}3.3 \\
3.1\end{array}$ & 15.3 & 0.375 & 0.11 \\
\hline & 11-8-F3.8-6\#3-i-2.5-3-14.5 & $\begin{array}{l}\text { A } \\
\text { B }\end{array}$ & 21.7 & 19.4 & 20 & 22.19 & $\begin{array}{l}2.4 \\
2.5\end{array}$ & 2.4 & $\begin{array}{l}3.2 \\
3.6\end{array}$ & 15.4 & 0.375 & 0.11 \\
\hline & (3@5.35)11-8-F3.8-0-i-2.5-3-14.5 & $\begin{array}{l}\text { A } \\
\text { B } \\
\text { C }\end{array}$ & 22.0 & 19.3 & 20 & 21.53 & $\begin{array}{c}2.8 \\
- \\
2.8 \\
\end{array}$ & 2.8 & $\begin{array}{l}3.6 \\
3.2 \\
3.2 \\
\end{array}$ & $\begin{array}{l}7.5 \\
7.6 \\
\end{array}$ & - & - \\
\hline & (3@5.35)11-8-F3.8-2\#3-i-2.5-3-14.5 & $\begin{array}{l}\text { A } \\
\text { B } \\
\text { C }\end{array}$ & 21.8 & 19.2 & 20 & 22.10 & $\begin{array}{c}2.5 \\
- \\
2.6\end{array}$ & 2.6 & $\begin{array}{l}3.3 \\
3.2 \\
3.3\end{array}$ & $\begin{array}{l}7.5 \\
7.8\end{array}$ & 0.375 & 0.11 \\
\hline & (3@5.35)11-8-F3.8-6\#3-i-2.5-3-14.5 & $\begin{array}{l}\text { A } \\
\text { B } \\
\text { C }\end{array}$ & 22.2 & 19.6 & 20 & 22.42 & $\begin{array}{c}2.8 \\
- \\
2.8\end{array}$ & 2.8 & $\begin{array}{l}3.1 \\
3.4 \\
3.5\end{array}$ & $\begin{array}{l}7.6 \\
7.6\end{array}$ & 0.375 & 0.11 \\
\hline
\end{tabular}


Table A.2 Cont. Comprehensive test results and data for beam-column joint specimens

\begin{tabular}{|c|c|c|c|c|c|c|c|c|c|c|c|}
\hline & Specimen & Head & $N$ & $\begin{array}{l}S_{t r}{ }^{*} \\
\text { in. }\end{array}$ & $\begin{array}{c}\boldsymbol{A}_{\boldsymbol{t t}} \\
\text { in. }^{2}\end{array}$ & $\begin{array}{l}\boldsymbol{d}_{\text {tro }} \\
\text { in. }\end{array}$ & $\begin{array}{c}\text { Stro }^{*} \\
\text { in. }\end{array}$ & $\begin{array}{l}\boldsymbol{A}_{\boldsymbol{a} \boldsymbol{b}} \\
\text { in. }^{2}\end{array}$ & $n$ & $\begin{array}{l}\boldsymbol{A}_{\boldsymbol{h s}} \\
\text { in. }^{2}\end{array}$ & $\begin{array}{l}\text { Long. } \\
\text { Reinf. } \\
\text { Layout }\end{array}$ \\
\hline \multirow{5}{*}{ Group 15} & 11-5a-F3.8-0-i-2.5-3-12 & $\begin{array}{l}\text { A } \\
\text { B }\end{array}$ & - & - & - & 0.5 & $\begin{array}{c}5 \\
(2.5) \\
\end{array}$ & 1.20 & 2 & 3.12 & A11 \\
\hline & 11-5a-F3.8-2\#3-i-2.5-3-12 & $\begin{array}{l}\mathrm{A} \\
\mathrm{B}\end{array}$ & 4 & $\begin{array}{c}8 \\
(6) \\
\end{array}$ & 0.22 & 0.5 & $\begin{array}{c}5 \\
(2.5) \\
\end{array}$ & 1.20 & 2 & 3.12 & A11 \\
\hline & 11-5a-F3.8-6\#3-i-2.5-3-12 & $\begin{array}{l}A \\
B\end{array}$ & 12 & $\begin{array}{c}4 \\
(2) \\
\end{array}$ & 0.66 & 0.5 & $\begin{array}{c}5 \\
(2.5) \\
\end{array}$ & 1.20 & 2 & 3.12 & A11 \\
\hline & 11-5a- F8.6-0-i-2.5-3-12 & $\begin{array}{l}\text { A } \\
B\end{array}$ & - & - & - & 0.5 & $\begin{array}{c}5 \\
(2.5) \\
\end{array}$ & 1.20 & 2 & 3.12 & A11 \\
\hline & 11-5a- F8.6-6\#3-i-2.5-3-12 & $\begin{array}{l}\text { A } \\
B\end{array}$ & 12 & $\begin{array}{c}4 \\
(2) \\
\end{array}$ & 0.66 & 0.5 & $\begin{array}{c}5 \\
(2.5) \\
\end{array}$ & 1.20 & 2 & 3.12 & A11 \\
\hline \multirow{9}{*}{ Group 16} & 8-8-F4.1-0-i-2.5-3-10-DB & $\begin{array}{l}A \\
B\end{array}$ & - & - & - & 0.5 & $\begin{array}{c}5 \\
(2.5) \\
\end{array}$ & 0.80 & 2 & 1.58 & A12 \\
\hline & 8-8-F9.1-0-i-2.5-3-10-DB & $\begin{array}{l}\text { A } \\
B\end{array}$ & - & - & - & 0.5 & $\begin{array}{c}5 \\
(2.5) \\
\end{array}$ & 0.80 & 2 & 1.58 & A12 \\
\hline & 8-8-F9.1-5\#3-i-2.5-3-10-DB & $\begin{array}{l}A \\
B\end{array}$ & 10 & $\begin{array}{c}5 \\
(2.5)\end{array}$ & 0.44 & 0.5 & $\begin{array}{c}5 \\
(2.5)\end{array}$ & 0.80 & 2 & 1.58 & A12 \\
\hline & 8-5-F4.1-0-i-2.5-3-10-DB & $\begin{array}{l}\text { A } \\
B\end{array}$ & - & - & - & 0.5 & $\begin{array}{c}5 \\
(2.5) \\
\end{array}$ & 0.80 & 2 & 1.58 & A12 \\
\hline & 8-5-F9.1-0-i-2.5-3-10-DB & $\begin{array}{l}A \\
B\end{array}$ & - & - & - & 0.5 & $\begin{array}{c}5 \\
(2.5)\end{array}$ & 0.80 & 2 & 1.58 & A12 \\
\hline & 8-5-F4.1-3\#4-i-2.5-3-10-DB & $\begin{array}{l}\text { A } \\
B\end{array}$ & 6 & $\begin{array}{l}7.5 \\
(6) \\
\end{array}$ & 0.40 & 0.5 & $\begin{array}{c}5 \\
(2.5) \\
\end{array}$ & 0.80 & 2 & 1.58 & A12 \\
\hline & 8-5-F9.1-3\#4-i-2.5-3-10-DB & $\begin{array}{l}A \\
B\end{array}$ & 6 & $\begin{array}{l}7.5 \\
(6)\end{array}$ & 0.40 & 0.5 & $\begin{array}{c}5 \\
(2.5)\end{array}$ & 0.80 & 2 & 1.58 & A12 \\
\hline & 8-5-F4.1-5\#3-i-2.5-3-10-DB & $\begin{array}{l}\text { A } \\
B\end{array}$ & 10 & $\begin{array}{c}5 \\
(2.5) \\
\end{array}$ & 0.44 & 0.5 & $\begin{array}{c}5 \\
(2.5) \\
\end{array}$ & 0.80 & 2 & 1.58 & A12 \\
\hline & 8-5-F9.1-5\#3-i-2.5-3-10-DB & $\begin{array}{l}\text { A } \\
\text { B }\end{array}$ & 10 & $\begin{array}{c}5 \\
(2.5) \\
\end{array}$ & 0.44 & 0.5 & $\begin{array}{c}5 \\
(2.5) \\
\end{array}$ & 0.80 & 2 & 1.58 & A12 \\
\hline \multirow{6}{*}{ Group 17} & 11-8-F3.8-0-i-2.5-3-14.5 & $\begin{array}{l}\text { A } \\
B\end{array}$ & - & - & - & 0.5 & $\begin{array}{c}5 \\
(2.5)\end{array}$ & 1.20 & 2 & 3.12 & A12 \\
\hline & 11-8-F3.8-2\#3-i-2.5-3-14.5 & $\begin{array}{l}\text { A } \\
\text { B }\end{array}$ & 4 & $\begin{array}{c}8 \\
(6) \\
\end{array}$ & 0.22 & 0.5 & $\begin{array}{c}5 \\
(2.5) \\
\end{array}$ & 1.20 & 2 & 3.12 & A12 \\
\hline & 11-8-F3.8-6\#3-i-2.5-3-14.5 & $\begin{array}{l}\text { A } \\
\text { B }\end{array}$ & 12 & $\begin{array}{c}4 \\
(2) \\
\end{array}$ & 0.66 & 0.5 & $\begin{array}{c}5 \\
(2.5) \\
\end{array}$ & 1.20 & 2 & 3.12 & A12 \\
\hline & (3@5.35)11-8-F3.8-0-i-2.5-3-14.5 & $\begin{array}{l}\mathrm{A} \\
\mathrm{B} \\
\mathrm{C}\end{array}$ & - & - & - & 0.5 & $\begin{array}{c}5 \\
(2.5)\end{array}$ & 1.20 & 3 & 4.68 & A13 \\
\hline & (3@5.35)11-8-F3.8-2\#3-i-2.5-3-14.5 & $\begin{array}{l}\mathrm{A} \\
\mathrm{B} \\
\mathrm{C}\end{array}$ & 4 & $\begin{array}{c}8 \\
(6)\end{array}$ & 0.22 & 0.5 & $\begin{array}{c}5 \\
(2.5)\end{array}$ & 1.20 & 3 & 4.68 & A14 \\
\hline & (3@5.35)11-8-F3.8-6\#3-i-2.5-3-14.5 & $\begin{array}{l}\text { A } \\
B \\
C\end{array}$ & 12 & $\begin{array}{c}4 \\
(2)\end{array}$ & 0.66 & 0.5 & $\begin{array}{c}5 \\
(2.5)\end{array}$ & 1.20 & 3 & 4.68 & A14 \\
\hline
\end{tabular}

\footnotetext{
${ }^{*}$ Value in parenthesis is the spacing between the first hoop and the center of the headed bar
} 
Table A.2 Cont. Comprehensive test results and data for beam-column joint specimens

\begin{tabular}{|c|c|c|c|c|c|c|c|c|c|c|}
\hline & Specimen & Head & $\begin{array}{c}\text { Failure } \\
\text { Type }\end{array}$ & $\begin{array}{c}\text { Lead (Head) } \\
\text { Slip } \\
\text { in. }\end{array}$ & $\begin{array}{l}\boldsymbol{T}_{\max } \\
\text { kips }\end{array}$ & $\begin{array}{c}\boldsymbol{f}_{\text {su,max }} \\
\text { ksi }\end{array}$ & $\begin{array}{l}\boldsymbol{T}_{\text {ind }} \\
\text { kips }\end{array}$ & $\begin{array}{l}\boldsymbol{T}_{\text {total }} \\
\text { kips }\end{array}$ & $\begin{array}{c}\boldsymbol{T} \\
\text { kips }\end{array}$ & $\begin{array}{l}\boldsymbol{f}_{\text {su }} \\
\mathrm{ksi}\end{array}$ \\
\hline \multirow{5}{*}{ Group 15} & 11-5a-F3.8-0-i-2.5-3-12 & $\begin{array}{l}\mathrm{A} \\
\mathrm{B}\end{array}$ & CB & $\begin{array}{l}0.025 \\
0.006\end{array}$ & $\begin{array}{l}54.2 \\
59.5 \\
\end{array}$ & $\begin{array}{l}34.7 \\
38.1 \\
\end{array}$ & $\begin{array}{l}54.2 \\
59.5 \\
\end{array}$ & 113.7 & 56.8 & 36.4 \\
\hline & 11-5a-F3.8-2\#3-i-2.5-3-12 & $\begin{array}{l}\text { A } \\
B\end{array}$ & CB & $\begin{array}{l}0.231 \\
0.007\end{array}$ & $\begin{array}{l}67.2 \\
67.4\end{array}$ & $\begin{array}{l}43.1 \\
43.2\end{array}$ & $\begin{array}{l}67.2 \\
67.4\end{array}$ & 134.6 & 67.3 & 43.1 \\
\hline & 11-5a-F3.8-6\#3-i-2.5-3-12 & $\begin{array}{l}\mathrm{A} \\
\mathrm{B}\end{array}$ & $\mathrm{CB} / \mathrm{FP}$ & $\begin{array}{l}0.007 \\
0.429 \\
\end{array}$ & $\begin{array}{l}77.4 \\
82.3 \\
\end{array}$ & $\begin{array}{l}49.6 \\
52.8 \\
\end{array}$ & $\begin{array}{l}77.4 \\
78.6 \\
\end{array}$ & 156.0 & 78.0 & 50.0 \\
\hline & 11-5a- F8.6-0-i-2.5-3-12 & $\begin{array}{l}\text { A } \\
\text { B }\end{array}$ & CB & $\begin{array}{l}0.202 \\
0.145\end{array}$ & $\begin{array}{l}63.7 \\
75.4\end{array}$ & $\begin{array}{l}40.8 \\
48.3\end{array}$ & $\begin{array}{l}63.7 \\
64.0\end{array}$ & 127.7 & 63.8 & 40.9 \\
\hline & 11-5a- F8.6-6\#3-i-2.5-3-12 & $\begin{array}{l}\mathrm{A} \\
\mathrm{B}\end{array}$ & CB & $\begin{array}{l}0.237 \\
0.250 \\
\end{array}$ & $\begin{array}{l}78.3 \\
80.2 \\
\end{array}$ & $\begin{array}{l}50.2 \\
51.4 \\
\end{array}$ & $\begin{array}{l}78.3 \\
80.2 \\
\end{array}$ & 158.4 & 79.2 & 50.8 \\
\hline \multirow{9}{*}{ Group 16} & 8-8-F4.1-0-i-2.5-3-10-DB & $\begin{array}{l}\mathrm{A} \\
\mathrm{B}\end{array}$ & CB & $\begin{array}{l}0.129 \\
0.065 \\
\end{array}$ & $\begin{array}{l}49.9 \\
50.5 \\
\end{array}$ & $\begin{array}{l}63.2 \\
63.9 \\
\end{array}$ & $\begin{array}{l}49.9 \\
50.5 \\
\end{array}$ & 100.3 & 50.2 & 63.5 \\
\hline & 8-8-F9.1-0-i-2.5-3-10-DB & $\begin{array}{l}\mathrm{A} \\
\mathrm{B}\end{array}$ & CB & $\begin{array}{l}0.010 \\
0.036 \\
\end{array}$ & $\begin{array}{l}47.4 \\
56.2 \\
\end{array}$ & $\begin{array}{l}60.0 \\
71.1 \\
\end{array}$ & $\begin{array}{l}47.4 \\
56.2 \\
\end{array}$ & 103.6 & 51.8 & 65.6 \\
\hline & 8-8-F9.1-5\#3-i-2.5-3-10-DB & $\begin{array}{l}\mathrm{A} \\
\mathrm{B}\end{array}$ & CB & $\begin{array}{l}0.012 \\
0.102 \\
\end{array}$ & $\begin{array}{l}65.5 \\
71.0 \\
\end{array}$ & $\begin{array}{l}82.9 \\
89.9 \\
\end{array}$ & $\begin{array}{l}65.5 \\
71.0 \\
\end{array}$ & 136.5 & 68.2 & 86.3 \\
\hline & 8-5-F4.1-0-i-2.5-3-10-DB & $\begin{array}{l}\mathrm{A} \\
\mathrm{B}\end{array}$ & $\mathrm{CB} / \mathrm{FP}$ & $\begin{array}{l}0.188 \\
0.322 \\
\end{array}$ & $\begin{array}{l}37.4 \\
44.4 \\
\end{array}$ & $\begin{array}{l}47.3 \\
56.2 \\
\end{array}$ & $\begin{array}{l}37.4 \\
43.9 \\
\end{array}$ & 81.3 & 40.6 & 51.4 \\
\hline & 8-5-F9.1-0-i-2.5-3-10-DB & $\begin{array}{l}\text { A } \\
B\end{array}$ & CB & $\begin{array}{l}0.061 \\
0.008\end{array}$ & $\begin{array}{l}42.6 \\
49.7\end{array}$ & $\begin{array}{l}53.9 \\
62.9\end{array}$ & $\begin{array}{l}39.0 \\
49.7\end{array}$ & 88.7 & 44.4 & 56.2 \\
\hline & 8-5-F4.1-3\#4-i-2.5-3-10-DB & $\begin{array}{l}\mathrm{A} \\
\mathrm{B}\end{array}$ & CB & $\begin{array}{l}0.081 \\
0.180 \\
\end{array}$ & $\begin{array}{l}60.6 \\
68.7 \\
\end{array}$ & $\begin{array}{l}76.7 \\
87.0 \\
\end{array}$ & $\begin{array}{l}60.6 \\
68.7 \\
\end{array}$ & 129.2 & 64.6 & 81.8 \\
\hline & 8-5-F9.1-3\#4-i-2.5-3-10-DB & $\begin{array}{l}\mathrm{A} \\
\mathrm{B}\end{array}$ & CB & $\begin{array}{l}0.017 \\
0.258 \\
\end{array}$ & $\begin{array}{l}62.4 \\
69.1 \\
\end{array}$ & $\begin{array}{l}79.0 \\
87.5 \\
\end{array}$ & $\begin{array}{l}62.4 \\
69.1 \\
\end{array}$ & 131.5 & 65.8 & 83.3 \\
\hline & 8-5-F4.1-5\#3-i-2.5-3-10-DB & $\begin{array}{l}\mathrm{A} \\
\mathrm{B}\end{array}$ & CB & $\begin{array}{l}0.019 \\
0.120 \\
\end{array}$ & $\begin{array}{l}63.2 \\
77.2 \\
\end{array}$ & $\begin{array}{l}80.0 \\
97.7 \\
\end{array}$ & $\begin{array}{l}63.2 \\
77.2 \\
\end{array}$ & 140.4 & 70.2 & 88.9 \\
\hline & 8-5-F9.1-5\#3-i-2.5-3-10-DB & $\begin{array}{l}\text { A } \\
\text { B }\end{array}$ & CB & $\begin{array}{l}0.120 \\
0.248\end{array}$ & $\begin{array}{l}66.8 \\
74.2\end{array}$ & $\begin{array}{l}84.6 \\
93.9\end{array}$ & $\begin{array}{l}66.8 \\
74.2\end{array}$ & 141.0 & 70.5 & 89.2 \\
\hline \multirow{6}{*}{ Group 17} & 11-8-F3.8-0-i-2.5-3-14.5 & $\begin{array}{l}\text { A } \\
\text { B }\end{array}$ & CB & $\begin{array}{l}0.123 \\
0.008\end{array}$ & $\begin{array}{l}79.4 \\
78.7\end{array}$ & $\begin{array}{l}50.9 \\
50.4\end{array}$ & $\begin{array}{l}79.4 \\
78.7\end{array}$ & 158.1 & 79.1 & 50.7 \\
\hline & 11-8-F3.8-2\#3-i-2.5-3-14.5 & $\begin{array}{l}\mathrm{A} \\
\mathrm{B}\end{array}$ & CB & $\begin{array}{l}0.591 \\
0.008 \\
\end{array}$ & $\begin{array}{l}87.8 \\
89.1 \\
\end{array}$ & $\begin{array}{l}56.3 \\
57.1 \\
\end{array}$ & $\begin{array}{l}87.8 \\
89.1 \\
\end{array}$ & 176.9 & 88.4 & 56.7 \\
\hline & 11-8-F3.8-6\#3-i-2.5-3-14.5 & $\begin{array}{l}\mathrm{A} \\
\mathrm{B} \\
\end{array}$ & CB & $\begin{array}{l}0.140 \\
0.178 \\
\end{array}$ & $\begin{array}{l}112.4 \\
112.9 \\
\end{array}$ & $\begin{array}{l}72.1 \\
72.4 \\
\end{array}$ & $\begin{array}{l}112.4 \\
112.9 \\
\end{array}$ & 225.3 & 112.7 & 72.2 \\
\hline & (3@5.35)11-8-F3.8-0-i-2.5-3-14.5 & $\begin{array}{l}\mathrm{A} \\
\mathrm{B} \\
\mathrm{C}\end{array}$ & CB & $\begin{array}{c}- \\
0.040 \\
-\end{array}$ & $\begin{array}{l}51.9 \\
54.9 \\
51.9 \\
\end{array}$ & $\begin{array}{l}33.3 \\
35.2 \\
33.3 \\
\end{array}$ & $\begin{array}{l}51.9 \\
54.9 \\
51.9 \\
\end{array}$ & 158.7 & 52.9 & 33.9 \\
\hline & (3@5.35)11-8-F3.8-2\#3-i-2.5-3-14.5 & $\begin{array}{l}\text { A } \\
\mathrm{B} \\
\mathrm{C}\end{array}$ & CB & $\begin{array}{c}- \\
0.260 \\
-\end{array}$ & $\begin{array}{l}74.0 \\
72.1 \\
71.6 \\
\end{array}$ & $\begin{array}{l}47.4 \\
46.2 \\
45.9 \\
\end{array}$ & $\begin{array}{l}74.0 \\
72.1 \\
71.6 \\
\end{array}$ & 217.7 & 72.6 & 46.5 \\
\hline & (3@5.35)11-8-F3.8-6\#3-i-2.5-3-14.5 & $\begin{array}{l}\text { A } \\
\text { B } \\
\text { C }\end{array}$ & CB & $\begin{array}{c}- \\
0.211 \\
0.292\end{array}$ & $\begin{array}{l}93.2 \\
85.3 \\
72.4\end{array}$ & $\begin{array}{l}59.7 \\
54.7 \\
46.4\end{array}$ & $\begin{array}{l}93.2 \\
85.3 \\
72.4\end{array}$ & 251.0 & 83.7 & 53.7 \\
\hline
\end{tabular}


Table A.2 Cont. Comprehensive test results and data for beam-column joint specimens

\begin{tabular}{|c|c|c|c|c|c|c|c|c|c|c|}
\hline & Specimen & Head & $\begin{array}{l}\boldsymbol{c}_{\boldsymbol{o}} \\
\text { in. }\end{array}$ & $A_{b r g}$ & $\begin{array}{l}\ell_{\text {eh }} \\
\text { in. }\end{array}$ & $\begin{array}{c}\text { } \text { eh,avg } \\
\text { in. }\end{array}$ & $\begin{array}{l}\boldsymbol{f}_{\boldsymbol{c m}} \\
\text { psi }\end{array}$ & $\begin{array}{l}\text { Age } \\
\text { days }\end{array}$ & $\begin{array}{l}\boldsymbol{d}_{\boldsymbol{b}} \\
\text { in. }\end{array}$ & $\begin{array}{c}\boldsymbol{A}_{\boldsymbol{b}} \\
\text { in. }^{2}\end{array}$ \\
\hline \multirow{4}{*}{ Group 17} & 8-8-O12.9-0-i-2.5-3-9.5 & $\begin{array}{l}\text { A } \\
B\end{array}$ & 1.0 & $12.9 A_{b}$ & $\begin{array}{l}9.75 \\
9.63\end{array}$ & 9.69 & 8800 & 21 & 1 & 0.79 \\
\hline & 8-8-S14.9-0-i-2.5-3-8.25 & $\begin{array}{l}\text { A } \\
B\end{array}$ & 1.0 & $14.9 A_{b}$ & $\begin{array}{l}8.25 \\
8.25\end{array}$ & 8.25 & 8800 & 21 & 1 & 0.79 \\
\hline & 8-8-O12.9-5\#3-i-2.5-3-9.5 & $\begin{array}{l}\text { A } \\
B\end{array}$ & 1.0 & $12.9 A_{b}$ & $\begin{array}{l}9.50 \\
9.25\end{array}$ & 9.38 & 8800 & 21 & 1 & 0.79 \\
\hline & 8-8-S14.9-5\#3-i-2.5-3-8.25 & $\begin{array}{l}A \\
B\end{array}$ & 1.0 & $14.9 A_{b}$ & $\begin{array}{l}8.25 \\
8.25 \\
\end{array}$ & 8.25 & 8800 & 21 & 1 & 0.79 \\
\hline \multirow{8}{*}{ Group 18} & 11-5-F3.8-0-i-2.5-3-12 & $\begin{array}{l}A \\
B\end{array}$ & 2.0 & $3.8 A_{b}$ & $\begin{array}{l}12.13 \\
12.13\end{array}$ & 12.13 & 5760 & 6 & 1.41 & 1.56 \\
\hline & 11-5-F3.8-6\#3-i-2.5-3-12 & $\begin{array}{l}A \\
B\end{array}$ & 2.0 & $3.8 A_{b}$ & $\begin{array}{l}12.50 \\
12.50 \\
\end{array}$ & 12.50 & 5760 & 6 & 1.41 & 1.56 \\
\hline & 11-5-F3.8-0-i-2.5-3-17 & $\begin{array}{l}\text { A } \\
B\end{array}$ & 2.0 & $3.8 A_{b}$ & $\begin{array}{l}17.50 \\
17.00\end{array}$ & 17.25 & 5760 & 6 & 1.41 & 1.56 \\
\hline & 11-5-F3.8-6\#3-i-2.5-3-17 & $\begin{array}{l}A \\
B\end{array}$ & 2.0 & $3.8 A_{b}$ & $\begin{array}{l}16.88 \\
17.00 \\
\end{array}$ & 16.94 & 5970 & 7 & 1.41 & 1.56 \\
\hline & 11-5- F8.6-0-i-2.5-3-14.5 & $\begin{array}{l}\text { A } \\
B\end{array}$ & 2.0 & $8.6 A_{b}$ & $\begin{array}{l}14.50 \\
14.50\end{array}$ & 14.50 & 5970 & 7 & 1.41 & 1.56 \\
\hline & 11-5- F8.6-6\#3-i-2.5-3-14.5 & $\begin{array}{l}\text { A } \\
B\end{array}$ & 2.0 & $8.6 A_{b}$ & $\begin{array}{l}14.50 \\
14.75\end{array}$ & 14.63 & 5970 & 7 & 1.41 & 1.56 \\
\hline & (3@5.35)11-5- F8.6-0-i-2.5-3-14.5 & $\begin{array}{l}\text { A } \\
\text { B } \\
\text { C }\end{array}$ & 2.0 & $8.6 A_{b}$ & $\begin{array}{l}14.38 \\
15.25 \\
14.50\end{array}$ & 14.71 & 6240 & 8 & 1.41 & 1.56 \\
\hline & (3@5.35)11-5- F8.6-6\#3-i-2.5-3-14.5 & $\begin{array}{l}\text { A } \\
B \\
C\end{array}$ & 2.0 & $8.6 A_{b}$ & $\begin{array}{l}14.75 \\
14.50 \\
14.38\end{array}$ & 14.54 & 6240 & 8 & 1.41 & 1.56 \\
\hline \multirow{8}{*}{ Group 19} & 11-12-O4.5-0-i-2.5-3-16.75 & $\begin{array}{l}\text { A } \\
B\end{array}$ & 1.3 & $4.5 A_{b}$ & $\begin{array}{l}17.13 \\
17.13\end{array}$ & 17.13 & 10860 & 36 & 1.41 & 1.56 \\
\hline & 11-12-S5.5-0-i-2.5-3-16.75 & $\begin{array}{l}\text { A } \\
B\end{array}$ & 1.5 & $5.5 A_{b}$ & $\begin{array}{l}16.75 \\
17.13\end{array}$ & 16.94 & 10120 & 37 & 1.41 & 1.56 \\
\hline & 11-12-04.5-6\#3-i-2.5-3-16.75 & $\begin{array}{l}A \\
B\end{array}$ & 1.3 & $4.5 A_{b}$ & $\begin{array}{l}16.75 \\
16.88 \\
\end{array}$ & 16.81 & 10860 & 37 & 1.41 & 1.56 \\
\hline & 11-12-S5.5-6\#3-i-2.5-3-16.75 & $\begin{array}{l}A \\
B\end{array}$ & 1.5 & $5.5 A_{b}$ & $\begin{array}{l}16.63 \\
17.00\end{array}$ & 16.81 & 10120 & 38 & 1.41 & 1.56 \\
\hline & (3@5.35)11-12-O4.5-0-i-2.5-3-16.75 & $\begin{array}{l}\mathrm{A} \\
\mathrm{B} \\
\mathrm{C}\end{array}$ & 1.3 & $4.5 A_{b}$ & $\begin{array}{l}16.88 \\
17.13 \\
16.75 \\
\end{array}$ & 16.92 & 10860 & 36 & 1.41 & 1.56 \\
\hline & (3@5.35)11-12-S5.5-0-i-2.5-3-16.75 & $\begin{array}{l}\text { A } \\
B \\
C\end{array}$ & 1.5 & $5.5 A_{b}$ & $\begin{array}{l}16.88 \\
17.00 \\
16.88\end{array}$ & 16.92 & 10120 & 38 & 1.41 & 1.56 \\
\hline & (3@5.35)11-12-O4.5-6\#3-i-2.5-3-16.75 & $\begin{array}{l}\text { A } \\
B \\
\text { C }\end{array}$ & 1.3 & $4.5 A_{b}$ & $\begin{array}{l}16.88 \\
17.13 \\
17.00\end{array}$ & 17.00 & 10860 & 37 & 1.41 & 1.56 \\
\hline & (3@5.35)11-12-S5.5-6\#3-i-2.5-3-16.75 & $\begin{array}{l}\text { A } \\
B \\
C\end{array}$ & 1.5 & $5.5 A_{b}$ & $\begin{array}{l}16.75 \\
17.00 \\
16.50\end{array}$ & 16.75 & 10120 & 38 & 1.41 & 1.56 \\
\hline
\end{tabular}


Table A.2 Cont. Comprehensive test results and data for beam-column joint specimens

\begin{tabular}{|c|c|c|c|c|c|c|c|c|c|c|c|c|}
\hline & Specimen & Head & $\begin{array}{c}\boldsymbol{b} \\
\text { in. }\end{array}$ & $\begin{array}{l}\boldsymbol{h} \\
\text { in. }\end{array}$ & $\begin{array}{l}\boldsymbol{h}_{\boldsymbol{c l}} \\
\text { in. }\end{array}$ & $\begin{array}{l}\boldsymbol{d}_{\text {eff }} \\
\text { in. }\end{array}$ & $\begin{array}{l}c_{s o} \\
\text { in. }\end{array}$ & $\begin{array}{c}\boldsymbol{C}_{\text {so }, \text { avg }} \\
\text { in. }\end{array}$ & $\begin{array}{l}c_{b c} \\
\text { in. }\end{array}$ & $\begin{array}{l}\boldsymbol{c}_{\boldsymbol{c h}} \\
\text { in. }\end{array}$ & $\begin{array}{l}\boldsymbol{d}_{t r} \\
\text { in. }\end{array}$ & $\begin{array}{l}\boldsymbol{A}_{t r, \boldsymbol{l}} \\
\text { in. }^{2}\end{array}$ \\
\hline \multirow{3}{*}{ Group 17} & 8-8-012.9-0-i-2.5-3-9.5 & $\begin{array}{l}\text { A } \\
\text { B }\end{array}$ & 16.9 & 14.3 & 10.25 & 12.31 & $\begin{array}{l}2.5 \\
2.5 \\
\end{array}$ & 2.5 & $\begin{array}{l}2.9 \\
3.0\end{array}$ & 10.9 & - & - \\
\hline & 8-8-S14.9-0-i-2.5-3-8.25 & $\begin{array}{l}\text { A } \\
B\end{array}$ & 17.3 & 14.3 & 10.25 & 11.97 & $\begin{array}{l}2.6 \\
2.6\end{array}$ & 2.6 & $\begin{array}{l}3.3 \\
3.3\end{array}$ & 11.0 & - & - \\
\hline & 8-8-S14.9-5\#3-i-2.5-3-8.25 & $\begin{array}{l}\text { A } \\
B\end{array}$ & 17.3 & 14.1 & 10.25 & 12.36 & $\begin{array}{l}2.8 \\
2.5\end{array}$ & 2.6 & $\begin{array}{l}3.1 \\
3.1\end{array}$ & 11.0 & 0.375 & 0.11 \\
\hline \multirow{6}{*}{ Group 18} & 11-5-F3.8-0-i-2.5-3-12 & $\begin{array}{l}\text { A } \\
B\end{array}$ & 21.7 & 17.0 & 20 & 21.66 & $\begin{array}{l}2.8 \\
2.5 \\
\end{array}$ & 2.6 & $\begin{array}{l}3.5 \\
3.5 \\
\end{array}$ & 15.0 & - & - \\
\hline & 11-5-F3.8-6\#3-i-2.5-3-17 & $\begin{array}{l}\text { A } \\
B\end{array}$ & 21.5 & 22.0 & 20 & 23.70 & $\begin{array}{l}2.6 \\
2.5\end{array}$ & 2.6 & $\begin{array}{l}3.8 \\
3.6\end{array}$ & 15.0 & 0.375 & 0.11 \\
\hline & 11-5- F8.6-0-i-2.5-3-14.5 & $\begin{array}{l}\text { A } \\
B\end{array}$ & 21.7 & 19.1 & 20 & 22.02 & $\begin{array}{l}2.5 \\
2.6 \\
\end{array}$ & 2.6 & $\begin{array}{l}3.3 \\
3.3 \\
\end{array}$ & 15.1 & - & - \\
\hline & 11-5- F8.6-6\#3-i-2.5-3-14.5 & $\begin{array}{l}\text { A } \\
B\end{array}$ & 21.5 & 19.5 & 20 & 22.74 & $\begin{array}{l}2.6 \\
2.6\end{array}$ & 2.6 & $\begin{array}{l}3.6 \\
3.4\end{array}$ & 14.9 & 0.375 & 0.11 \\
\hline & (3@5.35)11-5- F8.6-0-i-2.5-3-14.5 & $\begin{array}{l}\text { A } \\
B \\
C \\
\end{array}$ & 21.3 & 19.2 & 20 & 22.32 & $\begin{array}{c}2.6 \\
- \\
2.5 \\
\end{array}$ & 2.6 & $\begin{array}{l}3.4 \\
2.6 \\
3.3 \\
\end{array}$ & $\begin{array}{l}7.3 \\
7.5 \\
\end{array}$ & - & - \\
\hline & (3@5.35)11-5- F8.6-6\#3-i-2.5-3-14.5 & $\begin{array}{l}\mathrm{A} \\
\mathrm{B} \\
\mathrm{C} \\
\end{array}$ & 21.4 & 19.1 & 20 & 22.70 & $\begin{array}{c}2.5 \\
- \\
2.8 \\
\end{array}$ & 2.6 & $\begin{array}{l}3.0 \\
3.3 \\
3.4 \\
\end{array}$ & $\begin{array}{l}7.4 \\
7.4 \\
\end{array}$ & 0.375 & 0.11 \\
\hline \multirow{6}{*}{ Group 19} & 11-12-O4.5-6\#3-i-2.5-3-16.75 & $\begin{array}{l}\text { A } \\
\text { B }\end{array}$ & 21.7 & 23.1 & 20 & 23.12 & $\begin{array}{l}2.5 \\
2.8\end{array}$ & 2.6 & $\begin{array}{l}4.3 \\
4.1 \\
\end{array}$ & 15.0 & 0.375 & 0.11 \\
\hline & 11-12-S5.5-6\#3-i-2.5-3-16.75 & $\begin{array}{l}\text { A } \\
B\end{array}$ & 22.4 & 22.7 & 20 & 23.28 & $\begin{array}{l}2.6 \\
3.0\end{array}$ & 2.8 & $\begin{array}{l}3.3 \\
2.9\end{array}$ & 15.4 & 0.375 & 0.11 \\
\hline & (3@5.35)11-12-O4.5-0-i-2.5-3-16.75 & $\begin{array}{l}\mathrm{A} \\
\mathrm{B} \\
\mathrm{C}\end{array}$ & 21.8 & 22.9 & 20 & 22.48 & $\begin{array}{c}3.0 \\
- \\
2.5 \\
\end{array}$ & 2.8 & $\begin{array}{l}3.9 \\
3.6 \\
4.0 \\
\end{array}$ & $\begin{array}{l}7.5 \\
7.4 \\
\end{array}$ & - & - \\
\hline & (3@5.35)11-12-S5.5-0-i-2.5-3-16.75 & $\begin{array}{l}\text { A } \\
\text { B } \\
\text { C }\end{array}$ & 21.9 & 23.1 & 20 & 22.72 & $\begin{array}{c}2.8 \\
- \\
2.8 \\
\end{array}$ & 2.8 & $\begin{array}{l}3.5 \\
3.4 \\
3.5 \\
\end{array}$ & $\begin{array}{l}7.5 \\
7.5 \\
\end{array}$ & - & - \\
\hline & (3@5.35)11-12-O4.5-6\#3-i-2.5-3-16.75 & $\begin{array}{l}\text { A } \\
B \\
C\end{array}$ & 21.8 & 22.9 & 20 & 23.16 & $\begin{array}{c}2.5 \\
- \\
2.8\end{array}$ & 2.6 & $\begin{array}{l}3.9 \\
3.7 \\
3.8\end{array}$ & $\begin{array}{l}7.5 \\
7.6\end{array}$ & 0.375 & 0.11 \\
\hline & (3@5.35)11-12-S5.5-6\#3-i-2.5-3-16.75 & $\begin{array}{l}\text { A } \\
B \\
C\end{array}$ & 21.9 & 22.8 & 20 & 23.84 & $\begin{array}{c}2.6 \\
- \\
3.0\end{array}$ & 2.8 & $\begin{array}{l}3.3 \\
3.0 \\
3.5\end{array}$ & $\begin{array}{l}7.4 \\
7.5\end{array}$ & 0.375 & 0.11 \\
\hline
\end{tabular}


Table A.2 Cont. Comprehensive test results and data for beam-column joint specimens

\begin{tabular}{|c|c|c|c|c|c|c|c|c|c|c|c|}
\hline & Specimen & Head & $N$ & $\begin{array}{l}\boldsymbol{s}_{t r}{ }^{*} \\
\text { in. }\end{array}$ & $\begin{array}{l}\boldsymbol{A}_{t t} \\
\text { in. }^{2}\end{array}$ & $\begin{array}{l}\boldsymbol{d}_{\text {tro }} \\
\text { in. }\end{array}$ & $\begin{array}{l}\text { stro }^{*} \\
\text { in. }\end{array}$ & $\begin{array}{l}\boldsymbol{A}_{\boldsymbol{a b}} \\
\text { in. }^{2}\end{array}$ & $n$ & $\begin{array}{l}\boldsymbol{A}_{\boldsymbol{h s}} \\
\text { in. }^{2}\end{array}$ & $\begin{array}{l}\text { Long. } \\
\text { Reinf. } \\
\text { Layout }\end{array}$ \\
\hline \multirow{4}{*}{ Group 17} & 8-8-012.9-0-i-2.5-3-9.5 & $\begin{array}{l}\text { A } \\
B\end{array}$ & - & - & - & 0.5 & $\begin{array}{c}4.5 \\
(2.25)\end{array}$ & 0.80 & 2 & 1.58 & A6 \\
\hline & 8-8-S14.9-0-i-2.5-3-8.25 & $\begin{array}{l}\text { A } \\
B\end{array}$ & - & - & - & 0.5 & $\begin{array}{c}4.5 \\
(2.25)\end{array}$ & 0.80 & 2 & 1.58 & A6 \\
\hline & 8-8-012.9-5\#3-i-2.5-3-9.5 & $\begin{array}{l}\text { A } \\
B\end{array}$ & 10 & $\begin{array}{c}3 \\
(1.5)\end{array}$ & 0.66 & 0.5 & $\begin{array}{c}4.5 \\
(2.25)\end{array}$ & 0.80 & 2 & 1.58 & A6 \\
\hline & 8-8-S14.9-5\#3-i-2.5-3-8.25 & $\begin{array}{l}\text { A } \\
B\end{array}$ & 10 & $\begin{array}{c}3 \\
(1.5)\end{array}$ & 0.66 & 0.5 & $\begin{array}{c}4.5 \\
(2.25)\end{array}$ & 0.80 & 2 & 1.58 & A6 \\
\hline \multirow{8}{*}{ Group 18} & 11-5-F3.8-0-i-2.5-3-12 & $\begin{array}{l}\text { A } \\
B\end{array}$ & - & - & - & 0.5 & $\begin{array}{c}5 \\
(2.5)\end{array}$ & 1.20 & 2 & 3.12 & A6 \\
\hline & 11-5-F3.8-6\#3-i-2.5-3-12 & $\begin{array}{l}A \\
B\end{array}$ & 12 & $\begin{array}{c}4 \\
(2)\end{array}$ & 0.66 & 0.5 & $\begin{array}{c}5 \\
(2.5)\end{array}$ & 1.20 & 2 & 3.12 & A12 \\
\hline & 11-5-F3.8-0-i-2.5-3-17 & $\begin{array}{l}A \\
B\end{array}$ & - & - & - & 0.5 & $\begin{array}{c}6 \\
(3)\end{array}$ & 0.80 & 2 & 3.12 & A7 \\
\hline & 11-5-F3.8-6\#3-i-2.5-3-17 & $\begin{array}{l}\mathrm{A} \\
\mathrm{B}\end{array}$ & 12 & $\begin{array}{c}4 \\
(2) \\
\end{array}$ & 0.66 & 0.5 & $\begin{array}{c}6 \\
(3) \\
\end{array}$ & 0.80 & 2 & 3.12 & A7 \\
\hline & 11-5- F8.6-0-i-2.5-3-14.5 & $\begin{array}{l}A \\
B\end{array}$ & - & - & - & 0.5 & $\begin{array}{c}5 \\
(2.5)\end{array}$ & 1.20 & 2 & 3.12 & A12 \\
\hline & 11-5- F8.6-6\#3-i-2.5-3-14.5 & $\begin{array}{l}\text { A } \\
B\end{array}$ & 12 & $\begin{array}{c}4 \\
(2)\end{array}$ & 0.66 & 0.5 & $\begin{array}{c}5 \\
(2.5)\end{array}$ & 1.20 & 2 & 3.12 & A12 \\
\hline & (3@5.35)11-5- F8.6-0-i-2.5-3-14.5 & $\begin{array}{l}\text { A } \\
\text { B } \\
\text { C }\end{array}$ & - & - & - & 0.5 & $\begin{array}{c}5 \\
(2.5)\end{array}$ & 1.20 & 3 & 4.68 & A15 \\
\hline & (3@5.35)11-5- F8.6-6\#3-i-2.5-3-14.5 & $\begin{array}{l}\text { A } \\
B \\
C\end{array}$ & 12 & $\begin{array}{c}4 \\
(2)\end{array}$ & 0.66 & 0.5 & $\begin{array}{c}4.5 \\
(2.25)\end{array}$ & 1.20 & 3 & 4.68 & A15 \\
\hline \multirow{8}{*}{ Group 19} & 11-12-O4.5-0-i-2.5-3-16.75 & $\begin{array}{l}A \\
B\end{array}$ & - & - & - & 0.5 & $\begin{array}{c}4 \\
(2)\end{array}$ & 1.20 & 2 & 3.12 & A16 \\
\hline & $11-12-S 5.5-0-i-2.5-3-16.75$ & $\begin{array}{l}\text { A } \\
B\end{array}$ & - & - & - & 0.5 & $\begin{array}{c}4 \\
(2)\end{array}$ & 1.20 & 2 & 3.12 & A16 \\
\hline & 11-12-O4.5-6\#3-i-2.5-3-16.75 & $\begin{array}{l}\text { A } \\
\text { B }\end{array}$ & 12 & $\begin{array}{c}4 \\
(2) \\
\end{array}$ & 0.66 & 0.5 & $\begin{array}{c}4 \\
(2) \\
\end{array}$ & 1.20 & 2 & 3.12 & A16 \\
\hline & 11-12-S5.5-6\#3-i-2.5-3-16.75 & $\begin{array}{l}A \\
B\end{array}$ & 12 & $\begin{array}{c}4 \\
(2)\end{array}$ & 0.66 & 0.5 & $\begin{array}{c}4 \\
(2)\end{array}$ & 1.20 & 2 & 3.12 & A16 \\
\hline & (3@5.35)11-12-O4.5-0-i-2.5-3-16.75 & $\begin{array}{l}\text { A } \\
B \\
C\end{array}$ & - & - & - & 0.5 & $\begin{array}{c}4 \\
(2)\end{array}$ & 1.20 & 3 & 4.68 & A16 \\
\hline & (3@5.35)11-12-S5.5-0-i-2.5-3-16.75 & $\begin{array}{l}\text { A } \\
\text { B } \\
\text { C }\end{array}$ & - & - & - & 0.5 & $\begin{array}{c}4 \\
(2)\end{array}$ & 1.20 & 3 & 4.68 & A16 \\
\hline & (3@5.35)11-12-O4.5-6\#3-i-2.5-3-16.75 & $\begin{array}{l}\text { A } \\
B \\
C\end{array}$ & 12 & $\begin{array}{c}4 \\
(2)\end{array}$ & 0.66 & 0.5 & $\begin{array}{c}4 \\
(2)\end{array}$ & 1.20 & 3 & 4.68 & A16 \\
\hline & (3@5.35)11-12-S5.5-6\#3-i-2.5-3-16.75 & $\begin{array}{l}\text { A } \\
\text { B } \\
\text { C }\end{array}$ & 12 & $\begin{array}{c}4 \\
(2)\end{array}$ & 0.66 & 0.5 & $\begin{array}{c}4 \\
(2)\end{array}$ & 1.20 & 3 & 4.68 & A16 \\
\hline
\end{tabular}

${ }^{*}$ Value in parenthesis is the spacing between the first hoop and the center of the headed bar 
Table A.2 Cont. Comprehensive test results and data for beam-column joint specimens

\begin{tabular}{|c|c|c|c|c|c|c|c|c|c|c|}
\hline & Specimen & Head & $\begin{array}{c}\text { Failure } \\
\text { Type }\end{array}$ & $\begin{array}{c}\text { Lead (Head) } \\
\text { Slip } \\
\text { in. }\end{array}$ & $\begin{array}{l}\boldsymbol{T}_{\max } \\
\text { kips }\end{array}$ & $\begin{array}{c}\boldsymbol{f}_{\text {su,max }} \\
\text { ksi }\end{array}$ & $\begin{array}{l}\boldsymbol{T}_{\text {ind }} \\
\text { kips }\end{array}$ & $\begin{array}{l}\boldsymbol{T}_{\text {total }} \\
\text { kips }\end{array}$ & $\begin{array}{c}\boldsymbol{T} \\
\text { kips }\end{array}$ & $\begin{array}{l}f_{s u} \\
\mathrm{ksi}\end{array}$ \\
\hline \multirow{4}{*}{ Group 17} & 8-8-O12.9-0-i-2.5-3-9.5 & $\begin{array}{l}\text { A } \\
B\end{array}$ & CB & - & $\begin{array}{l}85.5 \\
84.9\end{array}$ & $\begin{array}{l}108.2 \\
107.5\end{array}$ & $\begin{array}{l}85.5 \\
84.9\end{array}$ & 170.5 & 85.2 & 107.8 \\
\hline & $8-8-S 14.9-0-\mathrm{i}-2.5-3-8.25$ & $\begin{array}{l}\mathrm{A} \\
\mathrm{B}\end{array}$ & $\mathrm{CB}$ & $\begin{array}{l}0.010 \\
0.187\end{array}$ & $\begin{array}{l}70.8 \\
71.0\end{array}$ & $\begin{array}{l}89.6 \\
89.9\end{array}$ & $\begin{array}{l}70.8 \\
71.0\end{array}$ & 141.8 & 70.9 & 89.7 \\
\hline & 8-8-012.9-5\#3-i-2.5-3-9.5 & $\begin{array}{l}\mathrm{A} \\
\mathrm{B}\end{array}$ & CB & $\begin{array}{l}0.237 \\
0.198\end{array}$ & $\begin{array}{l}84.4 \\
82.5\end{array}$ & $\begin{array}{l}106.8 \\
104.4\end{array}$ & $\begin{array}{l}84.4 \\
82.5\end{array}$ & 166.9 & 83.5 & 105.7 \\
\hline & 8-8-S14.9-5\#3-i-2.5-3-8.25 & $\begin{array}{l}\text { A } \\
\text { B }\end{array}$ & CB & $\begin{array}{l}0.197 \\
0.022\end{array}$ & $\begin{array}{l}87.2 \\
86.8\end{array}$ & $\begin{array}{l}110.4 \\
109.9\end{array}$ & $\begin{array}{l}87.2 \\
86.8\end{array}$ & 174.0 & 87.0 & 110.1 \\
\hline \multirow{8}{*}{ Group 18} & 11-5-F3.8-0-i-2.5-3-12 & $\begin{array}{l}\mathrm{A} \\
\mathrm{B}\end{array}$ & $\mathrm{CB}$ & $\begin{array}{l}0.140 \\
0.262 \\
\end{array}$ & $\begin{array}{l}68.7 \\
64.3 \\
\end{array}$ & $\begin{array}{l}44.0 \\
41.2 \\
\end{array}$ & $\begin{array}{l}68.7 \\
64.3 \\
\end{array}$ & 132.9 & 66.5 & 42.6 \\
\hline & 11-5-F3.8-6\#3-i-2.5-3-12 & $\begin{array}{l}\text { A } \\
\text { B }\end{array}$ & CB & $\begin{array}{l}0.041 \\
0.008\end{array}$ & $\begin{array}{l}88.2 \\
88.3\end{array}$ & $\begin{array}{l}56.5 \\
56.6\end{array}$ & $\begin{array}{l}88.2 \\
88.3\end{array}$ & 176.5 & 88.3 & 56.6 \\
\hline & 11-5-F3.8-0-i-2.5-3-17 & $\begin{array}{l}\mathrm{A} \\
\mathrm{B}\end{array}$ & $\mathrm{CB} / \mathrm{FP}$ & $\begin{array}{l}0.115 \\
0.015\end{array}$ & $\begin{array}{l}132.6 \\
132.9 \\
\end{array}$ & $\begin{array}{l}85.0 \\
85.2 \\
\end{array}$ & $\begin{array}{l}132.6 \\
132.9 \\
\end{array}$ & 265.5 & 132.7 & 85.1 \\
\hline & 11-5-F3.8-6\#3-i-2.5-3-17 & $\begin{array}{l}\text { A } \\
B\end{array}$ & CB & $\begin{array}{l}0.157 \\
0.051\end{array}$ & $\begin{array}{l}154.9 \\
148.9\end{array}$ & $\begin{array}{l}99.3 \\
95.4\end{array}$ & $\begin{array}{l}154.9 \\
148.9\end{array}$ & 303.7 & 151.9 & 97.4 \\
\hline & 11-5- F8.6-0-i-2.5-3-14.5 & $\begin{array}{l}\text { A } \\
B\end{array}$ & CB & $\begin{array}{l}0.005 \\
0.783\end{array}$ & $\begin{array}{l}83.6 \\
82.1\end{array}$ & $\begin{array}{l}53.6 \\
52.6\end{array}$ & $\begin{array}{l}83.6 \\
82.1\end{array}$ & 165.7 & 82.8 & 53.1 \\
\hline & 11-5- F8.6-6\#3-i-2.5-3-14.5 & $\begin{array}{l}\text { A } \\
\text { B }\end{array}$ & CB & $\begin{array}{l}0.144 \\
0.010\end{array}$ & $\begin{array}{l}113.9 \\
110.7\end{array}$ & $\begin{array}{l}73.0 \\
71.0\end{array}$ & $\begin{array}{l}113.9 \\
110.7\end{array}$ & 224.6 & 112.3 & 72.0 \\
\hline & (3@5.35)11-5- F8.6-0-i-2.5-3-14.5 & $\begin{array}{l}\mathrm{A} \\
\mathrm{B} \\
\mathrm{C}\end{array}$ & CB & $\begin{array}{c}- \\
0.013 \\
0.068 \\
\end{array}$ & $\begin{array}{l}66.5 \\
61.7 \\
67.1 \\
\end{array}$ & $\begin{array}{l}42.6 \\
39.6 \\
43.0 \\
\end{array}$ & $\begin{array}{l}66.5 \\
61.7 \\
67.1 \\
\end{array}$ & 195.4 & 65.1 & 41.7 \\
\hline & (3@5.35)11-5- F8.6-6\#3-i-2.5-3-14.5 & $\begin{array}{l}\mathrm{A} \\
\mathrm{B} \\
\mathrm{C}\end{array}$ & CB & $\begin{array}{c}- \\
0.287 \\
0.016 \\
\end{array}$ & $\begin{array}{l}68.8 \\
83.3 \\
74.9 \\
\end{array}$ & $\begin{array}{l}44.1 \\
53.4 \\
48.0 \\
\end{array}$ & $\begin{array}{l}68.8 \\
83.2 \\
74.9 \\
\end{array}$ & 226.9 & 75.6 & 48.5 \\
\hline \multirow{8}{*}{ Group 19} & $11-12-04.5-0-\mathrm{i}-2.5-3-16.75$ & $\begin{array}{l}\mathrm{A} \\
\mathrm{B}\end{array}$ & CB & $\begin{array}{l}0.032 \\
0.029\end{array}$ & $\begin{array}{l}168.4 \\
171.0\end{array}$ & $\begin{array}{l}107.9 \\
109.6\end{array}$ & $\begin{array}{l}168.3 \\
171.0\end{array}$ & 339.3 & 169.6 & 108.7 \\
\hline & 11-12-S5.5-0-i-2.5-3-16.75 & $\begin{array}{l}\text { A } \\
\text { B }\end{array}$ & CB & $\begin{array}{l}0.091 \\
0.215 \\
\end{array}$ & $\begin{array}{l}179.1 \\
172.7 \\
\end{array}$ & $\begin{array}{l}114.8 \\
110.7 \\
\end{array}$ & $\begin{array}{l}179.1 \\
172.7 \\
\end{array}$ & 351.9 & 175.9 & 112.8 \\
\hline & 11-12-04.5-6\#3-i-2.5-3-16.75 & $\begin{array}{l}\mathrm{A} \\
\mathrm{B}\end{array}$ & SB/FP & $\begin{array}{l}0.024 \\
0.022 \\
\end{array}$ & $\begin{array}{l}202.5 \\
200.7^{\ddagger}\end{array}$ & $\begin{array}{l}129.8 \\
128.7^{\ddagger} \\
\end{array}$ & $\begin{array}{l}202.5 \\
200.5\end{array}$ & 403.0 & 201.5 & 129.2 \\
\hline & 11-12-S5.5-6\#3-i-2.5-3-16.75 & $\begin{array}{l}\text { A } \\
B\end{array}$ & CB & $\begin{array}{l}0.028 \\
0.025\end{array}$ & $\begin{array}{l}206.1 \\
188.7\end{array}$ & $\begin{array}{l}132.1 \\
121.0\end{array}$ & $\begin{array}{l}206.1 \\
188.7\end{array}$ & 394.8 & 197.4 & 126.5 \\
\hline & (3@5.35)11-12-O4.5-0-i-2.5-3-16.75 & $\begin{array}{l}\mathrm{A} \\
\mathrm{B} \\
\mathrm{C}\end{array}$ & CB & $\begin{array}{c}- \\
0.003 \\
0.003\end{array}$ & $\begin{array}{c}109.3 \\
114.1 \\
98.7\end{array}$ & $\begin{array}{l}70.1 \\
73.1 \\
63.3\end{array}$ & $\begin{array}{c}107.6 \\
114.1 \\
98.7\end{array}$ & 320.4 & 106.8 & 68.5 \\
\hline & (3@5.35)11-12-S5.5-0-i-2.5-3-16.75 & $\begin{array}{l}\text { A } \\
\text { B } \\
\text { C }\end{array}$ & CB & $\begin{array}{l}- \\
- \\
-\end{array}$ & $\begin{array}{c}117.1 \\
93.8 \\
116.1 \\
\end{array}$ & $\begin{array}{l}75.1 \\
60.1 \\
74.4 \\
\end{array}$ & $\begin{array}{c}117.1 \\
93.8 \\
116.1 \\
\end{array}$ & 327.0 & 109.0 & 69.9 \\
\hline & (3@5.35)11-12-04.5-6\#3-i-2.5-3-16.75 & $\begin{array}{l}\text { A } \\
\text { B } \\
\text { C }\end{array}$ & CB & $\begin{array}{c}- \\
0.213 \\
0.145\end{array}$ & $\begin{array}{l}131.7 \\
131.8 \\
143.9\end{array}$ & $\begin{array}{l}84.4 \\
84.5 \\
92.2\end{array}$ & $\begin{array}{l}131.7 \\
131.8 \\
143.9\end{array}$ & 407.4 & 135.8 & 87.1 \\
\hline & (3@5.35)11-12-S5.5-6\#3-i-2.5-3-16.75 & $\begin{array}{l}\text { A } \\
\text { B } \\
\text { C }\end{array}$ & CB & $\begin{array}{c}- \\
0.095 \\
-\end{array}$ & $\begin{array}{l}155.9 \\
154.9 \\
150.6\end{array}$ & $\begin{array}{l}99.9 \\
99.3 \\
96.5\end{array}$ & $\begin{array}{l}155.9 \\
154.9 \\
150.6\end{array}$ & 461.3 & 153.8 & 98.6 \\
\hline
\end{tabular}

${ }^{\ddagger}$ No anchorage failure on the bar 
Table A.2 Cont. Comprehensive test results and data for beam-column joint specimens

\begin{tabular}{|c|c|c|c|c|c|c|c|c|c|c|}
\hline & Specimen & Head & $\begin{array}{l}c_{o} \\
\text { in. }\end{array}$ & $A_{b r g}$ & $\begin{array}{l}\ell \text { eh } \\
\text { in. }\end{array}$ & $\begin{array}{c}\ell \text { eh,avg } \\
\text { in. }\end{array}$ & $\begin{array}{l}\boldsymbol{f}_{\boldsymbol{c m}} \\
\mathrm{psi}\end{array}$ & $\begin{array}{l}\text { Age } \\
\text { days }\end{array}$ & $\begin{array}{l}\boldsymbol{d}_{\boldsymbol{b}} \\
\text { in. }\end{array}$ & $\begin{array}{l}A_{b} \\
\text { in. }^{2}\end{array}$ \\
\hline \multirow{8}{*}{ Group 20} & $11-5-04.5-0-\mathrm{i}-2.5-3-19.25$ & $\begin{array}{l}\text { A } \\
\text { B }\end{array}$ & 1.3 & $4.5 A_{b}$ & $\begin{array}{l}19.63 \\
19.25\end{array}$ & 19.44 & 5430 & 12 & 1.41 & 1.56 \\
\hline & 11-5-S5.5-0-i-2.5-3-19.25 & $\begin{array}{l}\text { A } \\
\text { B }\end{array}$ & 1.5 & $5.5 A_{b}$ & $\begin{array}{l}19.38 \\
19.38\end{array}$ & 19.38 & 6320 & 11 & 1.41 & 1.56 \\
\hline & 11-5-04.5-6\#3-i-2.5-3-19.25 & $\begin{array}{l}\text { A } \\
\text { B }\end{array}$ & 1.3 & $4.5 A_{b}$ & $\begin{array}{l}19.50 \\
19.75\end{array}$ & 19.63 & 5430 & 12 & 1.41 & 1.56 \\
\hline & 11-5-S5.5-6\#3-i-2.5-3-19.25 & $\begin{array}{l}\text { A } \\
\text { B }\end{array}$ & 1.5 & $5.5 A_{b}$ & $\begin{array}{l}19.13 \\
19.13\end{array}$ & 19.13 & 6320 & 13 & 1.41 & 1.56 \\
\hline & (3@5.35)11-5-O4.5-0-i-2.5-3-19.25 & $\begin{array}{l}\text { A } \\
\text { B } \\
\text { C }\end{array}$ & 1.3 & $4.5 A_{b}$ & $\begin{array}{l}19.50 \\
19.63 \\
19.38\end{array}$ & 19.50 & 5430 & 12 & 1.41 & 1.56 \\
\hline & (3@5.35)11-5-S5.5-0-i-2.5-3-19.25 & $\begin{array}{l}\text { A } \\
\text { B } \\
\text { C }\end{array}$ & 1.5 & $5.5 A_{b}$ & $\begin{array}{l}19.25 \\
19.38 \\
19.25\end{array}$ & 19.29 & 6320 & 11 & 1.41 & 1.56 \\
\hline & (3@5.35)11-5-O4.5-6\#3-i-2.5-3-19.25 & $\begin{array}{l}\text { A } \\
\text { B } \\
\text { C }\end{array}$ & 1.3 & $4.5 A_{b}$ & $\begin{array}{l}19.38 \\
19.63 \\
19.13\end{array}$ & 19.38 & 5430 & 13 & 1.41 & 1.56 \\
\hline & (3@5.35)11-5-S5.5-6\#3-i-2.5-3-19.25 & $\begin{array}{l}\text { A } \\
\text { B } \\
\text { C }\end{array}$ & 1.5 & $5.5 A_{b}$ & $\begin{array}{l}19.00 \\
19.38 \\
19.38\end{array}$ & 19.25 & 6320 & 13 & 1.41 & 1.56 \\
\hline
\end{tabular}

Table A.2 Cont. Comprehensive test results and data for beam-column joint specimens

\begin{tabular}{|c|c|c|c|c|c|c|c|c|c|c|c|c|}
\hline & Specimen & Head & $\begin{array}{c}\boldsymbol{b} \\
\text { in. }\end{array}$ & $\begin{array}{c}\boldsymbol{h} \\
\text { in. } \\
\end{array}$ & $\begin{array}{l}\boldsymbol{h}_{\boldsymbol{c l}} \\
\text { in. }\end{array}$ & $\begin{array}{l}\boldsymbol{d}_{\text {eff }} \\
\text { in. }\end{array}$ & $\begin{array}{l}c_{s o} \\
\text { in. }\end{array}$ & $\begin{array}{c}\boldsymbol{c}_{\boldsymbol{s o}, \text { avg }} \\
\text { in. } \\
\end{array}$ & $\begin{array}{l}c_{b c} \\
\text { in. }\end{array}$ & $\begin{array}{l}\boldsymbol{c}_{c h} \\
\text { in. }\end{array}$ & $\begin{array}{l}\boldsymbol{d}_{t r} \\
\text { in. }\end{array}$ & $\begin{array}{l}A_{t r, l} \\
\text { in. }^{2}\end{array}$ \\
\hline \multirow{8}{*}{ Group 20} & 11-5-O4.5-0-i-2.5-3-19.25 & $\begin{array}{l}\text { A } \\
\text { B }\end{array}$ & 21.9 & 25.6 & 20 & 24.09 & $\begin{array}{l}2.6 \\
2.8\end{array}$ & 2.7 & $\begin{array}{l}3.9 \\
4.3\end{array}$ & 15.1 & - & - \\
\hline & 11-5-S5.5-0-i-2.5-3-19.25 & $\begin{array}{l}\text { A } \\
\text { B }\end{array}$ & 22.0 & 25.4 & 20 & 24.17 & $\begin{array}{l}2.5 \\
3.0\end{array}$ & 2.8 & $\begin{array}{l}3.3 \\
3.3 \\
\end{array}$ & 15.1 & - & - \\
\hline & 11-5-O4.5-6\#3-i-2.5-3-19.25 & $\begin{array}{l}\text { A } \\
\text { B } \\
\end{array}$ & 21.7 & 25.6 & 20 & 24.70 & $\begin{array}{l}2.5 \\
2.8 \\
\end{array}$ & 2.6 & $\begin{array}{l}3.9 \\
3.7 \\
\end{array}$ & 15.0 & 0.375 & 0.11 \\
\hline & 11-5-S5.5-6\#3-i-2.5-3-19.25 & $\begin{array}{l}\text { A } \\
\text { B }\end{array}$ & 22.2 & 25.3 & 20 & 24.47 & $\begin{array}{l}2.8 \\
2.8\end{array}$ & 2.8 & $\begin{array}{l}3.4 \\
3.4\end{array}$ & 15.3 & 0.375 & 0.11 \\
\hline & (3@5.35)11-5-O4.5-0-i-2.5-3-19.25 & $\begin{array}{l}\text { A } \\
\text { B } \\
\text { C }\end{array}$ & 22.0 & 25.4 & 20 & 25.00 & $\begin{array}{c}2.8 \\
- \\
2.8 \\
\end{array}$ & 2.8 & $\begin{array}{l}3.8 \\
3.6 \\
3.9 \\
\end{array}$ & $\begin{array}{l}7.6 \\
7.5 \\
\end{array}$ & - & - \\
\hline & (3@5.35)11-5-S5.5-0-i-2.5-3-19.25 & $\begin{array}{l}\text { A } \\
\text { B } \\
\text { C } \\
\end{array}$ & 21.9 & 25.5 & 20 & 24.86 & $\begin{array}{c}2.8 \\
- \\
2.8 \\
\end{array}$ & 2.8 & $\begin{array}{l}3.5 \\
3.4 \\
3.5 \\
\end{array}$ & $\begin{array}{l}7.5 \\
7.5 \\
\end{array}$ & - & - \\
\hline & (3@5.35)11-5-04.5-6\#3-i-2.5-3-19.25 & $\begin{array}{l}\text { A } \\
\text { B } \\
\text { C }\end{array}$ & 21.8 & 25.4 & 20 & 25.50 & $\begin{array}{c}2.5 \\
- \\
2.6\end{array}$ & 2.6 & $\begin{array}{l}3.9 \\
3.6 \\
4.1\end{array}$ & $\begin{array}{l}7.6 \\
7.6\end{array}$ & 0.375 & 0.11 \\
\hline & (3@5.35)11-5-S5.5-6\#3-i-2.5-3-19.25 & $\begin{array}{l}\text { A } \\
\text { B } \\
\text { C } \\
\end{array}$ & 21.8 & 25.4 & 20 & 25.41 & $\begin{array}{c}2.6 \\
- \\
2.8 \\
\end{array}$ & 2.7 & $\begin{array}{l}3.6 \\
3.3 \\
3.3 \\
\end{array}$ & $\begin{array}{r}7.3 \\
7.8 \\
\end{array}$ & 0.375 & 0.11 \\
\hline
\end{tabular}


Table A.2 Cont. Comprehensive test results and data for beam-column joint specimens

\begin{tabular}{|c|c|c|c|c|c|c|c|c|c|c|c|}
\hline & Specimen & Head & $N$ & $\begin{array}{l}\boldsymbol{s}_{t r}^{*} \\
\text { in. }\end{array}$ & $\begin{array}{l}\boldsymbol{A}_{\boldsymbol{t t}} \\
\text { in. }^{2}\end{array}$ & $\begin{array}{l}\boldsymbol{d}_{\text {tro }} \\
\text { in. }\end{array}$ & $\begin{array}{l}\text { Stro }^{*} \\
\text { in. }\end{array}$ & $\begin{array}{l}A_{a b} \\
\text { in. }^{2}\end{array}$ & $n$ & $\begin{array}{l}\boldsymbol{A}_{\boldsymbol{h s}} \\
\text { in. }^{2}\end{array}$ & $\begin{array}{l}\text { Long. } \\
\text { Reinf. } \\
\text { Layout }\end{array}$ \\
\hline \multirow{8}{*}{ Group 20} & $11-5-04.5-0-\mathrm{i}-2.5-3-19.25$ & $\begin{array}{l}A \\
B\end{array}$ & - & - & - & 0.5 & $\begin{array}{c}4 \\
(2)\end{array}$ & 1.20 & 2 & 3.12 & A16 \\
\hline & 11-5-S5.5-0-i-2.5-3-19.25 & $\begin{array}{l}A \\
B\end{array}$ & - & - & - & 0.5 & $\begin{array}{c}4 \\
(2)\end{array}$ & 1.20 & 2 & 3.12 & A16 \\
\hline & 11-5-O4.5-6\#3-i-2.5-3-19.25 & $\begin{array}{l}\text { A } \\
B\end{array}$ & 12 & $\begin{array}{c}4 \\
(2)\end{array}$ & 0.66 & 0.5 & $\begin{array}{c}4 \\
(2)\end{array}$ & 1.20 & 2 & 3.12 & A16 \\
\hline & 11-5-S5.5-6\#3-i-2.5-3-19.25 & $\begin{array}{l}A \\
B\end{array}$ & 12 & $\begin{array}{c}4 \\
(2)\end{array}$ & 0.66 & 0.5 & $\begin{array}{c}4 \\
(2)\end{array}$ & 1.20 & 2 & 3.12 & A16 \\
\hline & (3@5.35)11-5-O4.5-0-i-2.5-3-19.25 & $\begin{array}{l}\mathrm{A} \\
\mathrm{B} \\
\mathrm{C}\end{array}$ & - & - & - & 0.5 & $\begin{array}{c}4 \\
(2)\end{array}$ & 1.20 & 3 & 4.68 & A16 \\
\hline & (3@5.35)11-5-S5.5-0-i-2.5-3-19.25 & $\begin{array}{l}A \\
B \\
C\end{array}$ & - & - & - & 0.5 & $\begin{array}{c}4 \\
(2)\end{array}$ & 1.20 & 3 & 4.68 & A16 \\
\hline & (3@5.35)11-5-O4.5-6\#3-i-2.5-3-19.25 & $\begin{array}{l}\text { A } \\
\text { B } \\
\text { C }\end{array}$ & 12 & $\begin{array}{c}4 \\
(2)\end{array}$ & 0.66 & 0.5 & $\begin{array}{c}4 \\
(2)\end{array}$ & 1.20 & 3 & 4.68 & A16 \\
\hline & (3@5.35)11-5-S5.5-6\#3-i-2.5-3-19.25 & $\begin{array}{l}\text { A } \\
B \\
C\end{array}$ & 12 & $\begin{array}{c}4 \\
(2)\end{array}$ & 0.66 & 0.5 & $\begin{array}{c}4 \\
(2)\end{array}$ & 1.20 & 3 & 4.68 & A16 \\
\hline
\end{tabular}

${ }^{*}$ Value in parenthesis is the spacing between the first hoop and the center of the headed bar

Table A.2 Cont. Comprehensive test results and data for beam-column joint specimens

\begin{tabular}{|c|c|c|c|c|c|c|c|c|c|c|}
\hline & Specimen & Head & $\begin{array}{c}\text { Failure } \\
\text { Type }\end{array}$ & $\begin{array}{c}\text { Lead (Head) } \\
\text { Slip } \\
\text { in. }\end{array}$ & $\begin{array}{l}\boldsymbol{T}_{\max } \\
\text { kips }\end{array}$ & $\begin{array}{c}\boldsymbol{f}_{\text {su,max }} \\
\text { ksi }\end{array}$ & $\begin{array}{l}\boldsymbol{T}_{\text {ind }} \\
\text { kips }\end{array}$ & $\begin{array}{l}\boldsymbol{T}_{\text {total }} \\
\text { kips }\end{array}$ & $\begin{array}{c}\boldsymbol{T} \\
\text { kips }\end{array}$ & $\begin{array}{l}\boldsymbol{f}_{\text {su }} \\
\mathrm{ksi}\end{array}$ \\
\hline \multirow{8}{*}{ Group 20} & $11-5-04.5-0-\mathrm{i}-2.5-3-19.25$ & $\begin{array}{l}\mathrm{A} \\
\mathrm{B}\end{array}$ & SB/FP & $\begin{array}{l}0.021 \\
0.128\end{array}$ & $\begin{array}{l}161.4 \\
154.4\end{array}$ & $\begin{array}{c}103.5 \\
99.0\end{array}$ & $\begin{array}{l}161.4 \\
154.3\end{array}$ & 315.7 & 157.9 & 101.2 \\
\hline & 11-5-S5.5-0-i-2.5-3-19.25 & $\begin{array}{l}\mathrm{A} \\
\mathrm{B}\end{array}$ & SB/FP & $\begin{array}{l}0.117 \\
0.095\end{array}$ & $\begin{array}{l}176.9 \\
176.8^{\ddagger}\end{array}$ & $\begin{array}{l}113.4 \\
113.3^{\ddagger}\end{array}$ & $\begin{array}{l}176.9 \\
176.7\end{array}$ & 353.6 & 176.8 & 113.3 \\
\hline & 11-5-O4.5-6\#3-i-2.5-3-19.25 & $\begin{array}{l}\text { A } \\
\text { B }\end{array}$ & $\mathrm{SB} / \mathrm{FP}$ & $\begin{array}{l}0.012 \\
0.036\end{array}$ & $\begin{array}{l}180.4^{\ddagger} \\
182.6\end{array}$ & $\begin{array}{l}115.6^{\ddagger} \\
117.1\end{array}$ & $\begin{array}{l}180.3 \\
182.6\end{array}$ & 362.9 & 181.4 & 116.3 \\
\hline & 11-5-S5.5-6\#3-i-2.5-3-19.25 & $\begin{array}{l}\text { A } \\
B\end{array}$ & $\mathrm{SB} / \mathrm{FP}$ & $\begin{array}{l}0.316 \\
0.147\end{array}$ & $\begin{array}{l}191.5^{\ddagger} \\
187.7\end{array}$ & $\begin{array}{l}122.8^{\ddagger} \\
120.3\end{array}$ & $\begin{array}{l}191.5 \\
187.7\end{array}$ & 379.2 & 189.6 & 121.5 \\
\hline & (3@5.35)11-5-O4.5-0-i-2.5-3-19.25 & $\begin{array}{l}\text { A } \\
B \\
C\end{array}$ & CB & $\begin{array}{c}0.001 \\
- \\
-\end{array}$ & $\begin{array}{l}132.5 \\
127.5 \\
126.0\end{array}$ & $\begin{array}{l}84.9 \\
81.7 \\
80.8 \\
\end{array}$ & $\begin{array}{l}132.5 \\
127.5 \\
126.0\end{array}$ & 386.0 & 128.7 & 82.5 \\
\hline & (3@5.35)11-5-S5.5-0-i-2.5-3-19.25 & $\begin{array}{l}\text { A } \\
\text { B } \\
\text { C }\end{array}$ & $\mathrm{CB} / \mathrm{BS}$ & $\begin{array}{c}- \\
0.321 \\
0.105\end{array}$ & $\begin{array}{l}137.3 \\
140.5 \\
134.9\end{array}$ & $\begin{array}{l}88.0 \\
90.1 \\
86.5\end{array}$ & $\begin{array}{l}137.3 \\
140.4 \\
134.5\end{array}$ & 412.2 & 137.4 & 88.1 \\
\hline & (3@5.35)11-5-O4.5-6\#3-i-2.5-3-19.25 & $\begin{array}{l}\text { A } \\
\text { B } \\
\text { C }\end{array}$ & CB & $\begin{array}{c}- \\
- \\
0.042\end{array}$ & $\begin{array}{l}137.4 \\
137.1 \\
150.7\end{array}$ & $\begin{array}{l}88.1 \\
87.9 \\
96.6\end{array}$ & $\begin{array}{l}137.4 \\
137.1 \\
150.7\end{array}$ & 425.1 & 141.7 & 90.8 \\
\hline & (3@5.35)11-5-S5.5-6\#3-i-2.5-3-19.25 & $\begin{array}{l}\text { A } \\
\text { B } \\
\text { C }\end{array}$ & CB & $\begin{array}{c}- \\
- \\
0.020\end{array}$ & $\begin{array}{l}151.6 \\
157.4 \\
149.5\end{array}$ & $\begin{array}{c}97.2 \\
100.9 \\
95.8\end{array}$ & $\begin{array}{l}151.6 \\
157.4 \\
149.5\end{array}$ & 458.6 & 152.9 & 98.0 \\
\hline
\end{tabular}

${ }^{\ddagger}$ No anchorage failure on the bar 